\title{
35TH ANNUAL SCIENTIFIC MEETING OF THE CANADIAN PAIN SOCIETY: ABSTRACTS
}

\author{
WEDNESDAY MAY 21, 2014 \\ SCIENTIFIC PROGRAM, DAY ONE \\ 9:00 am - 9:45 am
}

1

KEYNOTE SPEAKER: MARY ELLEN JEANS LECTURE

LOST IN SPACE? DISRUPTION AND TREATMENT OF CORTICAL MAPS OF SPACE AND BODY IN PEOPLE WITH CHRONIC PAIN

Chair: Jeffrey $S$ Mogil

EP Taylor Professor of Pain Studies, Canada Research Chair in the Genetics of Pain (Tier I), Department of Psychology and Alan Edwards Centre for Pain Research, McGill University, Montreal, Quebec

Invited Speaker: G Lorimer Moseley

Professor of Clinical Neurosciences, Foundation Chair in Physiotherapy \& NHMRC Principal Research Fellow; The University of South Australia, Adelaide \& Neuroscience Research Australia, Sydney, Australia

The brain holds maps of the body and the space around it. These maps subserve the regulation and protection of our body, and the space around it, both physiological and psychologically. That is, these maps are integrated with motor, sensory, and homeostatic functions as well as with the feelings we have of our body - its perceived size, location and temperature; that we own it and have agency over it. A growing body of literature suggests that many of these maps are disrupted in people with chronic pain. Our conventional understanding of how these maps would predict that their disruption simply reflects disrupted peripheral input- a purely 'bottom-up' phenomenon. However, recent experiments clearly reveal 'topdown' effects as well, which implies that disrupted cortical maps of space and body might contribute to the development or maintenance of chronic pain. That would raise the tantalizing possibility that these disrupted maps might be viable targets for treatment. Indeed, several treatments have been developed and preliminary results appear promising. This lecture will discuss the current state of research in this area, from the studies that underpin the idea of 'training the brain' for chronic pain, to the current state of evidence for their effectiveness.

Learning Objectives:

1. To understand the idea of cortical maps of the body and space around it.

2. To understand the evidence that these cortical maps are disrupted in people with chronic pain.

3. To understand the current evidence concerning treatments that aim to correct these disruptions as a way of treating chronic pain.

\section{Session 101: 10:45 am - 12:15 pm}

\section{2}

\section{PAIN IN VULNERABLE POPULATIONS: WHAT IS KNOWN AND WHERE TO GO NEXT?}

Chair: Line Caes

IWK Health Centre, Centre for Pediatric Pain Research, Halifax,

Nova Scotia

Speakers: Line Caes, Maria Glowacka, Lucia Gagliese

WORKSHOP OBJECTIVE:

To identify challenges associated with research on and treatment of pain in vulnerable samples across the life span, including children, women and older patients.

Learning Objectives:

1. Providing an overview of trends and contributions in the field of pediatric pain research over the last 35 years.
2. Discussing the prevalence and psychosocial predictors of genito-pelvic pain in women during pregnancy and three months postpartum.

3. Examining the profile of risk factors and resiliencies important for adjustment to pain among older adults.

2A

\section{AN OVERVIEW OF HOW PEDIATRIC PAIN RESEARCH HAS GROWN SINCE 1975}

Line Caes

Centre for Pediatric Pain Research, IWK Health Centre, Halifax, Nova Scotia

The field of pediatric pain research has grown tremendously since the first articles on the topic were published in the 1970-80's, yet no research has been conducted to systematically examine the trends and contributions to the pediatric pain literature since the early 1990's. The presentation will focus on a study that employs bibliometric analysis to quantitatively analyze scientific literature in order to document the impact and trends in pediatric pain research. The goal of the current bibliometric analysis is to provide an overview of research on pediatric pain published in peer-reviewed journals between 1975 and 2010. A title search, conducted in Web of Science in January 2013 by using developmental and pain-related search terms, resulted in a total of 7677 articles. Two individuals independently screened these titles to ensure eligibility for inclusion (ie, only original research articles and reviews related to pediatric pain with an English abstract were retained) resulting into 5693 articles to be included in the analysis. Moreover, $82 \%$ of the articles were published since 1990, indicating a substantial increase of pediatric pain publications since the last bibliometric analysis. The abstracts of the selected articles have been retrieved and are currently being coded on authors' country, citation score, type of journal and article, objective, age and type of sample, and pain stimulus. The results of this bibliometric analysis will provide a comprehensive examination of important trends and contributions in the field of pediatric pain research over the last 35 years and highlight areas in need of more research.

\section{B}

\section{PREVALENCE OF GENITO-PELVIC PAIN IN PREGNANCY AND THREE MONTHS POSTPARTUM AND THE PROSPECTIVE IMPACT OF FEAR-AVOIDANCE}

Maria Glowacka

Dalhousie University, Halifax, Nova Scotia

There is limited knowledge regarding the presence, onset, and location of genito-pelvic pain in pregnancy and postpartum. Further, greater fear-avoidance (pain-related anxiety, catastrophizing, hypervigilance to pain) has been associated with increased genital pain intensity in women, unrelated to childbirth. These relationships have not been examined in postpartum populations, or prospectively. We examined the prevalence, onset, and location of genito-pelvic pain during pregnancy and at three months postpartum, and the impact of fear-avoidance in pregnancy on the intensity and unpleasantness of genito-pelvic pain at three months postpartum. First-time expectant mothers $(n=150)$ completed measures of pain-related anxiety, catastrophizing and hypervigilance to pain. Those reporting genito-pelvic pain in pregnancy and/or at three months postpartum completed measures of the onset, location, intensity, and unpleasantness of their pain. Of 150 women, 73/150 $(49 \%)$ reported genito-pelvic pain in pregnancy. The pain resolved for 43 of 73 women and persisted for 30 of 73 , and 11 women reported a new onset of genito-pelvic pain after childbirth. Pain-related anxiety in pregnancy was the only independent predictor of both the intensity and unpleasantness of genito-pelvic pain postpartum. Results suggest that about half of women may develop genito-pelvic pain during pregnancy, but that this pain will resolve for many, persist for about a third, and a significant subset of women will newly develop pain after childbirth. Pain-related anxiety in pregnancy may increase the risk for greater postpartum genito-pelvic pain. Findings may help health care providers identify and offer early intervention to women who are at risk for experiencing this pain postpartum. 


\section{C}

MORE THAN JUST A NUMBER: HETEROGENEITY AND VULNERABILITY TO PAIN AMONG OLDER PEOPLE

\section{Lucia Gagliese}

York University and University Health Network, Toronto, Ontario

Pain is highly prevalent among older people in the community and across care settings. It has been repeatedly shown that older people are at risk for under-treatment of postoperative, chronic nonmalignant and cancer pain. Unrelieved pain in older people is associated with negative impacts across many domains of function and quality of life. However, older people are not a homogeneous group. In fact, heterogeneity in function and adaptation increase with age. As such, rather than thinking of all people over a certain age - an age that has yet to be agreed upon by researchers - as members of a vulnerable group, it may be more heuristic to begin to ask what the profile of biopsychosocial vulnerabilities is that increases some older people's risk for pain, poor adaptation and under-treatment. Conversely, it is also important to ask what resiliencies may operate as buffers of the impact of pain on function and quality of life in other older people. This presentation will consider these questions in light of emerging data about the role of age-related factors, such as health status, social context, and cognitive and psychological function, in pain interference and adaptation. It will be suggested that understanding the risks and buffers of poor pain outcomes may be more informative in guiding pain assessment and management than simple chronological age.

\section{Session 102: 10:45 am - 12:15 pm}

\section{3}

\section{THE BIOPSYCHOSOCIAL MODEL OF PAIN: WHERE ARE} WE TODAY?

\section{Chair: Fernando Cervero}

McGill University, Allan Edwards Centre for Research on Pain, Montreal, Quebec

Speakers: Fernando Cervero, Christine T Chambers,

Thomas Hadjistavropoulos

WORKSHOP OBJECTIVE:

To offer a critical discussion of the current status of the biopsychosocial model of pain, with consideration of progress and challenges in our understanding of biological, psychological, and social factors in pain.

Learning Objectives:

1. To describe the evolution and theoretical foundations of the biopsychosocial model of pain.

2. To consider progress and challenges in the study of various biological, psychological, and social factors and their integration in pain research.

3. To summarize research gaps and recommend directions for future research applying the biopsychosocial model of pain.

\section{A}

THE "BIO" IN THE BIOPSYCHOSOCIAL MODEL: CURRENT CHALLENGES

Fernando Cervero

McGill University, Alan Edwards Centre for Research on Pain,

Montreal, Quebec

It is generally acknowledged that human pain is a multifaceted phenomenon resulting from complex interactions between neurobiological mechanisms and psychological and social factors. This interpretation calls for an integrated approach to the treatment of pain, addressing not only the biological components but also the psychological and social elements that determine the final pain perception. From a purely neurobiological point of view there are several important components that determine the perception of pain. One is the interaction between afferent inputs so that the signals that reach the brain are always the result of modulation, both segmental and descending, between nociceptive and non-nociceptive pathways. A second factor is the development of pain chronicity, a process that transforms a useful alarm signal into a meaningless and unpleasant sensation. Chronicity is the result of the plasticity of the nociceptive system.
The third component is the genetic makeup of the individual, a factor thought to be key in the development of chronic pain states. These biological components of pain perception will be discussed as part of a wider discussion on the current state of the biopsychosocial model of pain.

\section{B}

\section{NOT PRACTICING WHAT WE PREACH: CHALLENGES IN PUTTING THE BIOPSYCHOSOCAL MODEL OF PAIN INTO PRACTICE IN PEDIATRIC PAIN RESEARCH}

\section{Christine T Chambers}

Departments of Pediatrics and Psychology, Dalhousie University and Centre for Pediatric Pain Research, IWK Health Centre, Halifax, Nova Scotia

Despite widespread acceptance of the importance of a biopsychosocial model in studying pain, research that exemplifies this truly integrated approach is scarce. Using pediatric pain as an example, where research continues to focus on biological, psychological, and social factors in isolation of one another, barriers (eg, practical, ethical, disciplinary) to conducting research within an integrative biopsychosocial framework will be discussed. Research examining the role of psychological factors in children's pain will be summarized, including the role of psychological variables in influencing pain perception in children as well as the efficacy of psychological interventions for improving pediatric pain. The lack of proper integration with relevant biological and social factors will be highlighted and potential strategies for enhancing true biopsychosocial research in pediatric pain will be discussed

\section{C}

\section{THE THEORETICAL FOUNDATIONS OF THE BIOPSYCHOSOCIAL FORMULATION OF PAIN: EMPHASIS ON SOCIAL/CONTEXTUAL FACTORS}

Thomas Hadjistavropoulos

Department of Psychology and Centre on Aging and Health, University of Regina, Regina, Saskatchewan

The history and evolution of the 'biopsychosocial model' of pain will be summarized, with an emphasis on the role of social/contextual factors in the pain experience and with attention to the testability of the 'model'. It is my contention that the 'biopsychosocial model' of pain is best construed as a framework, that allows us to summarize the literature and underscores recognition of the importance of biological and psychosocial parameters in the pain experience, rather than as a model per se. The framework is too broad to be testable using traditional scientific methodology that requires specificity of hypotheses. That is, while the biopsychosocial formulation allows for very broad predictions (eg, that social factors are influential), it does not allow for the generation of specific hypotheses. Specific models, falling under the broad biopsychosocial formulation (eg, the fear avoidance model), are testable and have generated falsifiable predictions. Nonetheless, biopsychosocial conceptualizations of pain are consistent with the gate control theory and have encouraged research that generated conclusive evidence in support of social/contextual influences on the pain experience. Much of this research, however, is analogue in nature and/or involves healthy volunteers. As such, there is a need for more investigations in naturalistic settings to support the findings of the analogue investigations. Specific future research directions will be discussed. 


\section{4} COMBINATION PHARMACOTHERAPY FOR PAIN: GENERAL
CONCEPTS, PRE-CLINICAL AND CLINICAL PERSPECTIVES Chair: Laura S Stone McGill University, Faculty of Dentistry, Alan Edwards Centre for Research on Pain, Departments of Pharmacology, Anesthesiology and Neurology \& Neurosurgery, Faculty of Medicine, Montreal, Quebec

Speakers: Laura S Stone, Ian Gilron, George L Wilcox WORKSHOP OBJECTIVES:

Individual pain-relievers provide incomplete relief and can produce undesired and dose-limiting side effects. The use of multimodal analgesic techniques can offer distinct advantages over single drug treatments. In some cases, combination therapies produce analgesia at lower doses, thus potentially reducing undesired side effects. However, there is also evidence of combinations that result in more serious side effects without improved pain management.

Participants will first be introduced to general principles of drug interactions, the potential potential advantages and limitations of polypharmacy and current hypotheses.

Learning Objectives:

After the presentations participants will be able to:

1. Discuss the potential clinical advantages of analgesic drug combinations.

2. Discuss the limitations of analgesic drug combinations.

3. Explain recent advances in our understanding of the mechanisms underlying synergistic drug interactions.

\section{$4 \mathrm{~A}$}

\section{COMBINATION PHARMACOTHERAPY FOR PAIN: GENERAL CONCEPTS}

\section{Laura S Stone}

McGill University, Faculty of Dentistry, Alan Edwards Centre for Research on Pain, Departments of Pharmacology, Anesthesiology and Neurology \& Neurosurgery, Faculty of Medicine, Montreal, Quebec

In this presentation, general concepts related to drug interactions, their measurement and their impact on potency, efficacy and therapeutic window will be introduced. Pre-clinical studies examining combination treatment with a $a_{2}$-adrenergic and opioid receptor agonists will be used to illustrate the potential utility of combination therapy in pain management.

\section{B}

\section{COMBINATION PHARMACOTHERAPY FOR PAIN: CLINICAL PERSPECTIVES}

\section{Ian Gilron}

Queens University, Director of Clinical Pain Research, Departments of Anesthesiology \& Perioperative Medicine, Biomedical \& Molecular Sciences, and Center for Neuroscience Studies, Queen's University, Kingston General Hospital, Kingston, Ontario

While polypharmacy can be beneficial, it can also be the source of more serious side-effects. Dr Gilron will review recent and ongoing clinical studies evaluating the efficacy and side-effect profiles of analgesic drug combinations in comparison to each individual drug. Issues to consider when using drug combinations in pain management will be discussed. Suggestions for future research that allow for more comprehensive testing of other analgesic combinations for pain management will be presented.

\section{$4 \mathrm{C}$}

\section{COMBINATION PHARMACOTHERAPY FOR PAIN: PRE-CLINICAL PERSPECTIVES}

\section{George L Wilcox}

University of Minnesota, Twin Cities Medical School, Departments of Neuroscience, Pharmacology and Dermatology, Minneapolis, Minnesota, USA

Opioid and a $\mathrm{a}_{2}$-adrenergic agonists are potent analgesic drugs and their analgesic effects can synergize when co-administered. These supra-additive interactions are potentially beneficial clinically; by increasing efficacy and/ or reducing the total drug dose required to produce sufficient pain relief, undesired side effects can be minimized. However, opioid and az-adrenergic combination therapies remain underutilized clinically despite a large body of pre-clinical evidence describing their synergistic interaction. One possible obstacle to the translation of pre-clinical findings to clinical applications is a lack of understanding of the mechanisms underlying the synergistic interactions between these two drug classes. Dr. Wilcox will present current hypotheses surrounding the cellular mechanisms mediating opioid-adrenergic synergy, including PKC signalling and receptor oligomerization. The implications of these findings for clinical applications and drug discovery will be discussed.

\section{Session 104: 10:45 am - 12:15 pm}

\section{5}

\section{ADDRESSING AND OVERCOMING PHYSICAL ACTIVITY BARRIERS FOR INDIVIDUALS WITH MUSCULOSKELETAL PAIN}

Chair: Timothy $\mathbf{H}$ Wideman

School of Medicine, Johns Hopkins University, Baltimore, Maryland, USA

Speakers: Timothy H Wideman, Katherine Harman, Neil Pearson WORKSHOP OBJECTIVE:

The objectives of this symposium are threefold:

To review the role of activity-related pain, psychological factors and pain sensitization as potential barriers to increasing physical activity and participating in activity-based interventions.

To present a clinical approach designed to help patients increase physical activity by integrating behaviour change techniques within a patient selfmanagement model.

To delineate specific clinical interventions and implementation strategies for facilitating activity-related behaviour change within primary care settings.

Learning Objectives:

1. To understand the relationship between activity-related pain, psychological factors and pain sensitization and their role as barriers to activity-based interventions.

2. To consider two perspectives (client and clinician) regarding the adoption of exercise and develop a clinical management strategy for addressing each of them.

3. To implement specific interventions and educational strategies within primary care settings to address and overcome barriers to functional recovery.

\section{A}

WHEN MORE ACTIVITY MEANS MORE PAIN: THE ROLE OF ACTIVITY-RELATED PAIN AS A BARRIER TO EXERCISE

Timothy $\mathbf{H}$ Wideman

School of Medicine, Johns Hopkins University, Baltimore,

Maryland, USA

Clinical practice guidelines commonly recommend activity-based interventions for chronic musculoskeletal pain. Participation in such treatments is typically associated with meaningful reductions in levels of pain and disability. Many patients, however, decline or discontinue such treatments due to increasing levels of pain as they engage in physical activity. Activity-related pain is thus an important barrier to treatment success and 
disability reduction and accounting for individual variance in this factor may be an important step in improving patient outcomes following activity-based interventions. The purpose of this presentation is to review the literature addressing activity-related pain and to discuss implications for clinical practice and future research. The presentation will begin with a review of normal responses to physical activity, including exercise-induced hypoalgesia, and desired therapeutic outcomes to activity-based interventions, such as increased strength, flexibility and cardiovascular fitness. Next, we will review the literature addressing a sensitized or problematic response to physical activity. Recent research suggests that certain individuals with musculoskeletal conditions experience increased pain sensitivity (eg, decreased pain thresholds) following bouts of exercise (ie, exercise-induced hyperalgesia) and that pain intensity summates with repeated or sustained physical activity (ie, summation of activity-related pain). Related research suggests that psychological factors and clinical indices of central sensitization are linked to increased sensitivity to activity-related pain. Next, we will review emerging clinical approaches for measuring increasing pain during physical activity, which include questionnaires and behavioral measures. The presentation will conclude with a discussion of the implication for clinical practice and future research.

\section{B}

\section{HOW CAN WE HELP OUR PATIENTS IN PAIN TO EXERCISE?}

Katherine Harman

School of Physiotherapy, Dalhousie University, Halifax, Nova Scotia

Although there is ample evidence of the benefits of exercise and also for the benefits of exercise in painful conditions, health providers have a challenge to get their patients to engage in activity and exercise. Whether a client engages in an exercise program likely depends on what they are expecting at the beginning; how important the outcome is to them and their confidence in doing the work - elements central to patient selfmanagement and health behaviour change theories. We can improve our clinical effectiveness by thinking about this while we deliver our interventions. What is going on in the patient's mind while the clinician is introducing an exercise program? Linking what the patient values to exercise will help the patient see the need to perform the exercises. It is important to identify barriers such as fear of re-injury and poor neuromuscular coordination. Then work towards solutions while building self-efficacy. Provide opportunities for patients to practice. Exercising in groups can be efficient, but also effective because of the effect of others on learning and behaviour change. Evidence-based exercise programs will not help our patients to improve, unless they do it.

\section{C}

\section{INCREASING PHYSICAL ACTIVITY AND FACILITATING RECOVERY IN PRIMARY CARE SETTINGS}

\section{Neil Pearson}

University of British Columbia, Vancouver, British Columbia

Addressing barriers to recovery is often a focus of interdisciplinary pain management programs, but commonly overlooked in primary care settings. While ample research suggests that early return to function is a key factor in recovery from pain conditions, there is limited literature to guide primary care practitioners in how to best address barriers to recovery. Based on evidence related to persistent pain, neuroscience and clinical experiences, guidelines have been developed to enhance the role of primary care practitioners in both the prevention and management of barriers to functional recovery.

This session describes and demonstrates interventions for use in primary care settings, such as brief pain science education, relaxed breathing, and implementation planning. These interventions will be related to previously discussed behaviour change strategies and barriers to physical activity. Strategies for immediate implementation within clinical settings will be discussed.
Session 105: 1:30 pm - 3:00 pm

\section{6}

\section{THE IMPACT OF PAIN, FEAR, AND PHYSIOLOGICAL RESPONSES TO NEEDLE PROCEDURES ACROSS THE LIFESPAN}

Chair: Anna Taddio

University of Toronto, Toronto, Ontario

Speakers: Anna Taddio, Blaine Ditto, Katelynn Boerner WORKSHOP OBJECTIVE:

Overarching vision of addressing needle fears and related pain through prevention and treatment efforts, as well as understanding the mechanisms that provoke and underlie negative reactions to needle procedures.

Learning Objectives:

1. Describe the prevalence, nature, and interactions of needle pain and fears, and the impact of needle fears on public health.

2. Understand the psychological, physiological, and intervention aspects related to needle-related vasovagal reactions and the role of pain as a stimulus for such reactions.

3. Describe the impact of needle anxiety on the implementation of procedural pain management strategies, and the outcomes of treating needle anxiety with a cognitive-behavioural framework.

\section{A}

\section{NEEDLE FEARS AND PUBLIC HEALTH: CONSEQUENCES FOR PAIN}

\section{Anna Taddio}

University of Toronto and The Hospital for Sick Children, Toronto, Ontario

Needle fears are highly prevalent across the lifespan. There is an important relationship between procedural pain and needle fears, as inadequate pain management may result in the classical conditioning of a fear response to needle procedures. Conversely, increased anxiety during a painful procedure has also been shown to increase the pain and distress associated with the procedure, and may impact the implementation of pain management interventions. There are numerous negative outcomes that inadequate management of needle pain and fears may have on children's health (eg, impact on health service delivery, parent and child satisfaction with medical care, immunization avoidance, long-term morbidity and mortality). This presentation will describe the prevalence of needle fears across the lifespan, the challenges that needle pain and fear pose to families experiencing needle procedures, as well as the consequences that such efforts may have for health care avoidance behaviour from a public health perspective. Pain has been found to be an important factor in willingness to undergo immunizations, and parental attitudes towards immunization pain and the subsequent impact this has on child fear will be reviewed. Additionally, recent knowledge translation efforts to reduce pain and fear in children receiving routine needle procedures will be highlighted.

\section{B}

\section{NEEDLE FEARS AND VASOVAGAL REACTIONS IN YOUNG ADULT BLOOD DONORS \\ Blaine Ditto}

McGill University, Department of Psychology, Montreal, Quebec

Despite their safety and widespread use in medical procedures, needles produce a fascinating and problematic array of psychological and physiological responses. In addition to pain, this can include a paralyzing sense of dread and vasovagal symptoms that range from mild dizziness to syncope. Vasovagal reactions can be triggered by pain though the relationship is complex. This presentation will discuss research suggesting that the distinctive symptoms produced by needles are related partly to an evolutionarily-based fear of blood loss and an 'adaptive' inhibition of cardiovascular activity. Results from several studies of young adult blood donors will be discussed. While North American blood donors are, by definition, volunteers, people give blood for many reasons and there is considerable 
variability in needle and other fears. The standardized procedures of blood donation also make blood clinics a useful environment to study interactions among fear, pain, vasovagal reactions, and avoidance behaviour (at least in terms of subsequent volunteering). Research concerning the effects of the muscle-tensing technique, Applied Tension, on fear, pain, vasovagal symptoms, and subsequent blood clinic attendance will also be discussed.

\section{C}

\section{IMPLEMENTING PROCEDURAL PAIN INTERVENTIONS IN CHILDREN WITH NEEDLE FEARS}

Katelynn E Boerner

Dalhousie University \& IWK Health Centre, Department of Psychology \& Neuroscience (Dalhousie University); Centre for Pediatric Pain Research (IWK Health Centre), Halifax, Nova Scotia Needle procedures are a painful and distressing experience for many children. There is a strong evidence base in favour of the efficacy of psychological interventions for reducing pain and distress during needle procedures. However, children with needle phobias are often excluded from this research, and offer a unique challenge to the implementation of evidence-based strategies before, during, and after a needle procedure. Many of the pre-existing challenges in implementing procedural pain management strategies (eg, environmental barriers, practical issues) are compounded by the presence of a particularly fearful child. This talk will describe the impact of needle anxiety on the implementation of procedural pain interventions, and will review of the protocol and pilot outcomes of a five-session cognitive-behavioural treatment group for children and parents that aimed to prepare children with needle anxiety for upcoming needle-related procedures. Practical suggestions for managing needlerelated anxiety in a number of settings in which painful needle procedures occur (eg, school immunization clinics, acute care settings, outpatient clinics) will be discussed.

\section{Session 106: 1:30 pm - 3:00 pm}

\section{7}

\section{MEDICAL CANNABIS AND THE NEW REGULATIONS: A MODERATED DIALOGUE BETWEEN PATIENT AND PHYSICIAN}

\section{Chair: Mark A Ware}

Anesthesia and Family Medicine, Alan Edwards Pain Management Unit, McGill University Health Centre, Montreal, Quebec Speakers: Mark A Ware, Terry Bremner, Ruth Dubin WORKSHOP OBJECTIVE:

This unique symposium will bring together a patient, a family physician and a researcher to discuss the complex issues of the medical use of cannabis. On April 1, 2014, Health Canada will implement the new Marijuana for Medical Purposes Regulations. These regulations will change the way medical cannabis is produced, analyzed, and distributed. This symposium will present the perspectives of a patient who is already a medical cannabis user, a family physician faced with concerns and questions about cannabis and the new program, moderated by a researcher and educator familiar with the drug and the regulations

Learning Objectives:

1. Describe the main components of the new marijuana for medical purposes regulations.

2. Access sources of information on the mechanisms, risks and benefits of cannabis in pain management.

3. Discuss use of cannabis with patients asking about medical cannabis.

\section{A}

\section{EXPLAINING THE MARIJUANA FOR MEDICAL PURPOSES REGULATIONS}

Mark Ware

Anesthesia and Family Medicine, Alan Edwards Pain Management Unit, McGill University Health Centre, Montreal, Quebec

This presentation will set the stage for the interactive symposium by providing a brief history of Canada's medical cannabis regulations and the recent transition to a new regulatory framework. An overview of the state of the new regulations will be provided and at the end of the session, this speaker will return to review the resources and tools available to health care professionals to guide them in this complex and controversial area.

\section{B}

WHERE WILL I GET MY MEDICATION AFTER APRIL 1, 2014? Terry Bremner

Patient with pain, Chronic Pain Association of Canada

Do you know how hard it was to first get my permit and documents to possess and use cannabis? I tried most all the other prescribed pain medications with very little success and a number of side effects. The road was long in trying to establish some form of relief from this complex disease called chronic pain. Thinking back to when I first applied for my permit I had to have two pain specialists and my own report to submit. This was very difficult as the wait times to see just one pain specialist in our country is pushing two years. In the mean time I had to buy my product off of the street while not always knowing who I bought it from and who grew it. It's been a tough rough road hiding around corners or buildings trying to conceal my identity from this illegal situation.

I did research and investigated how cannabis has been proven to help people that live with chronic pain, giving me the opportunity to have some relief and be able to become more active in my own life.

But not all cannabis is the same! I have used some cannabis that didn't affect me at all. Then, I have had some that made me feel like a couch potato and kept me away from my pool therapy for weeks. I wasn't able to keep up to commitments.

It took years to find the correct plant ingredient for my pain. It was the Sativa strand of cannabis that worked best for me. It gave me the energy to make it through my daily therapy, it also allowed me to assist in mowing the yard. Sativa has also given me the strength to volunteer to coach minor football. I guess in April 2014 I won't have a choice where I can find the medication that works best for me? I guess I will have to take what is given to me and not what I asked for? Buyer beware when you have to purchase unidentifiable medication from the only guy on the street. Where do I go?

\section{C}

\section{WHAT HAS THE GOVERNMENT DONE?}

Ruth Dubin

Department of Family Medicine (adjunct), Queens University, Kingston, Ontario

I really don't have a clue what the government has done. I completed very few medical cannabis licenses before they changed things. So an explanation of what the system was like before and what it is like now would be really helpful. The CFPC will likely want to make some sort of statement to the members about the implications of the change in the laws and we will not be able to do that without expert opinion. As always there will be the inadequate evidence for effectiveness argument, forgetting that we work with real patients doing $\mathrm{n}$ of one trials every day. But what are the risks and the benefits? What is safe medical use versus misuse and dependence. Can you take the same look at cannabis as you can at opiates, ie, what is problematic use, what are aberrant behaviours around cannabis? What is the safest way for someone who medically uses cannabis to ingest it? What are the risks of having easy access to cannabis for developing brains (teens)? People will worry about the societal impact of any change in legislation. Will the legislation put illegal pot growers out of business and make it easier to prevent under age users from getting it? Will it put the Mob out of business? 
Session 107: 1:30 pm - 3:00 pm

\section{8} WHEN THE TRACTOR WON'T STOP, TRY
DISCONNECTING THE FUEL LINE: THE PERNICIOUS
EFFECTS OF CONCOMITANT DEPRESSION AND ANXIETY
AND CHRONIC PAIN AND WHAT YOU CAN DO ABOUT IT Chair: Saifudin Rashiq

Speakers: Mark Simmonds, Pravesh Vallabh, Bruce Dick WORKSHOP OBJECTIVE:

Refractory chronic pain states in adults and children are often associated with clinically significant depression and/or anxiety. In this session, we will share our own institution's cross-sectional data demonstrating strong links between these phenomena in both adult and pediatric populations. Then, our tame psychiatrist will deliver a focused review of rational pharmacotherapy for these two common mental health issues. We hope to engage the audience in a lively discussion about the pros and cons of pharmacotherapy versus other modalities. Come with an open mind: If you don't know all about depression and anxiety at the beginning of the session, you surely will as the session grinds along through the 90 minutes to its conclusion.

Learning Objectives:

1. Attendees will hear about the measureable and clinically significant effects of depression and anxiety on outcomes from our group treatment strategies for early-and end-stage chronic pain in adults.

2. Attendees will learn more about best practice strategies for dealing with these pathologies in children with chronic pain, with particular emphasis on the role of the physician within the biopsychosocial model.

3. Attendees will receive a practical, clinical update on the use of drugs for the treatment of depression and anxiety in adults with chronic pain, and explore the obvious paradox that the two radically divergent treatment strategies for the two different age groups creates.

\section{A}

\section{"10 MINUTES OF YOUR TIME"}

\section{Mark Simmonds}

Department of Anesthesiology and Pain Medicine, University of Alberta, Edmonton, Alberta

This presentation will discuss the outcomes and implications of physician based educational methods for children and adolescents with complex chronic pain.

A brief, neurologically-oriented education intervention aimed at reducing the fear-avoidance beliefs of adolescent chronic pain patients was carried out. Our study was intended to simulate the limited amount of time physicians have to explain educational information during a consultation session. Feedback was collected from patients using a manipulation check and self-report questionnaires to see whether 1) neurologically-oriented education sessions were practical and relevant for chronic pain patients, and 2) whether brief education sessions reduced patients' fear-avoidance beliefs. The notable effects of this brief intervention on pain acceptance, fear of pain and movement, pain-related disability, and mental health will be discussed.

\section{B}

MEDICAL MANAGEMENT OF PAIN, DEPRESSION, AND ANXIETY IN CHRONIC PAIN

Pravesh Vallabh

Departments of Psychiatry \& Anesthesiology and Pain Medicine, University of Alberta, Edmonton, Alberta

The use of psychotropic medication has been established as a potentially useful pain management treatment modality for patients who experience mood disturbance concurrently with chronic pain. The neurobiological pathways for pain, mood, and sleep that are targeted by these medications will be discussed along with the rationale for their use. The evidence for how to most effectively use these medications within the treatment regime for these disorders will be presented. Finally, the crossover between pain and the functional spectrum of chronic pain syndromes including chronic widespread pain and fibromyalgia will also be reviewed.

\section{C}

\section{PSYCHOLOGICAL MANAGEMENT OF PAIN, DEPRESSION, AND ANXIETY: METHODS AND 10-YEAR OUTCOMES}

\section{Bruce Dick}

Departments of Anesthesiology and Pain Medicine \& Psychiatry, University of Alberta, Edmonton, Alberta

The findings from longitudinal studies of a 10-year adult multidisciplinary pain management program and a five-year pain management program for children and adolescents that followed patients for five years will be discussed. Outcome data will be presented for the Pain 101 program along with lessons learned for managing individuals across the lifespan with complex chronic pain syndromes. Practical strategies for program creation and management will be presented for attendees who practice pain management across a variety of clinical settings.

\section{Session 108: 1:30 pm - 3:00 pm}

\section{9}

\section{DATA BLITZ}

Chair: Marsha Campbell-Yeo

Faculty of Health Professions, School of Nursing; Department of Pediatrics \& Centre for Pediatric Pain Research, Dalhousie University; IWK Health Centre, Halifax, Nova Scotia

\section{NEW DEDICATED COURSE ON PAIN IN A MASTER'S LEVEL PHYSIOTHERAPY PROGRAM}

(Oral Presentation - Data Blitz)

Kadija Perreault ${ }^{1}$, Hélène Moffet ${ }^{1,2}$, Nathalie Mathieu ${ }^{1,3}$ Jean-François Roux ${ }^{1,3}$, Isabelle Savard ${ }^{1}$, Richard Debigaré ${ }^{1,4}$ ${ }^{1}$ Faculté de médecine, Université Laval; ${ }^{2}$ Centre interdisciplinaire de recherche en réadaptation et intégration sociale (CIRRIS), Institut de réadaptation en déficience physique de Québec; ${ }^{3}$ Centre Hospitalier Universitaire Affilié (CHAU) Hôtel-Dieu de Lévis, Centre de santé et de services sociaux (CSSS) Alphonse-Desjardins, Lévis; ${ }^{4}$ Centre de recherche de l'Institut universitaire en cardiologie et pneumologie de Québec

AIM: Research in the last decades has put forward the lack of time devoted to pain in healthcare providers' basic training, including physiotherapists, to whom people experiencing pain often turn for relief. At Université Laval, a new course dedicated to pain has been integrated into the master's physiotherapy program. This presentation describes its development, implementation and evaluation.

METHODS: The $30 \mathrm{~h}$ mandatory course aims to enable physiotherapy students to plan strategies for pain prevention and management for people of all ages, considering its multidimensional nature. The course includes a mix of lectures and workshops given by practicing clinicians and was developed based on IASP curricula and program needs. The course was evaluated using student surveys, discussions with educators and students' results on an end-of-training Objective Structured Clinical Examination (OSCE) station.

RESULTS: In the two years the course has been given, students appreciated the course $(98 \%[n=80])$ and reported on the value of acquiring painrelated knowledge, notably psychosocial issues and how to address them in practice. Challenges identified by students and educators included covering this complex subject within time-constraints and integrating practical learning activities. Students' results on the OSCE station demonstrated that most students were successful in demonstrating the expected skills and behaviours (mean performance $72 \pm 12 \%$ ) in a simulation environment. CONCLUSIONS: The integration of a dedicated course on pain in a master's physiotherapy program fills a gap in the training of future physiotherapists. Work is underway to continue improving this training, assess its impact, and adapt it to emerging evidence in the field. 


\section{A}

\section{ONLINE MINDFULNESS-BASED STRESS REDUCTION FOR THE MANAGEMENT OF CHRONIC HEADACHE PAIN IN ADULTS}

Angeline Traynor ${ }^{1}$, Brian McGuire ${ }^{1,2}$, Jonathan Egan ${ }^{1}$

${ }^{1}$ School of Psychology; ${ }^{2}$ Centre for Pain Research, National

University of Ireland, Galway, Ireland

AIM: To evaluate the efficacy of an online mindfulness based stress management program tailored for adults with chronic headache.

METHODS: A total of 221 participants (18 to 65 years of age) took part in a one group, pre-test to post-test internet based intervention for chronic headache management. An internet based program offering tailored mindfulness-based techniques and practical advice for self-management of chronic headache was developed (http://www.headachemanagement.org). A different theme and mindfulness exercise was introduced in each week of the six week program including: information about chronic headache, progressive muscle relaxation, guided visualization, sleep and headache onset relaxation. The primary treatment outcomes were pain severity and pain interference (Brief Pain Inventory), anxiety and depression (Hospital Anxiety and Depression Scale), headache impact (Headache Impact Test-6), medication intake and programme uptake. All participants selfreported their chronic headache diagnosis, they were predominantly tension-type headache and chronic migraine. Recruitment, data collection, data analyses were all performed online.

RESULTS: Of those completing the program ( $\mathrm{n}=71), 78 \%$ were female. Findings demonstrated clinically significant pre-to-post reductions in pain severity $(\mathrm{r}=0.69)$, pain interference $(\mathrm{r}=0.59)$, anxiety $(\mathrm{r}=0.66)$, depression $(\mathrm{r}=0.51)$, headache impact $(\mathrm{r}=0.53)$ and medication intake $(\mathrm{r}=0.54)$. Preto-post reduction in healthcare service treatment-seeking behaviour was not statistically significant. Attrition was considerably higher than expected at $70 \%$.

CONCLUSIONS: If the problem of participant attrition can be addressed, the results suggest that a relatively short online mindfulness-based selfmanagement program may be clinically effective for people with chronic headache, with benefits observed in pain severity and interference, anxiety and depression, and medication usage. Further research to assess these effects in a larger sample is underway. Indications of efficacy must be considered with caution, because they are based on an uncontrolled pre-post design.

\section{B}

\section{INCIDENCE OF INTRAVENOUS PATIENT CONTROLLED ANALGESIA AND CONTROLLED RELEASE OPIOIDS INITIATION FOLLOWING TOTAL KNEE ARTHROPLASTY} Arlene Buzon-Tan $^{1,2}$, Susan Walker ${ }^{1,2}$, Kyle Kirkham ${ }^{2}$

${ }^{1}$ Acute Pain Service; 2 Department of Anesthesia \& Pain Management, Toronto Western Hospital, University of Toronto, Toronto, Ontario

AIM: The objective of this quality assurance review is to describe the postoperative pain management practices of the Acute Pain Service at our tertiary care academic hospital (Toronto Western Hospital) in the total knee arthroplasty (TKA) population. In particular, we sought to determine the percentage of patients who required initiation of intravenous patient controlled analgesia (IV PCA) and controlled release (CR) opiates.

METHODS: A prospective quality assurance audit of 500 consecutive patients undergoing TKA was conducted. Current clinical practice includes spinal anesthesia with intrathecal morphine, intra-articular local anesthetic infiltration, oral opioids as needed, acetaminophen and NSAIDS. We examined the frequency of initiation of rescue IV PCA and CR opiate use. Preoperative opiate medication use was determined using patient records and interviews. Opioid tolerance was defined as the daily consumption of $30 \mathrm{mg}$ oral morphine or more.

RESULTS: Of the opiate-naive patients, 58 of 451 (12.9\%) required IV PCA, and CR opiates were administered in 35 of 451 (7.8\%). In the opioid tolerant patients, 28 of $49(57.1 \%)$ had IV PCA initiated, and 34 of $49(69.4 \%)$ received long acting opioids.

CONCLUSIONS: Given the extremely low requirement for IV PCA and $\mathrm{CR}$ opiate initiation in the opiate naive patients observed, the data supports our institutional standard of using principally oral immediate release opiate therapy as part of a multimodal regimen. While valuable, the use of IV PCA and CR opiates may be avoided on a routine basis for opiate naïve patients from a safety standpoint. The opiate tolerant population may require increased flexibility in the initiation of these modalities.

\section{C}

SAFETY PROFILE OF DOXEPIN 3 MG AND 6 MG IN ELDERLY INSOMNIA PATIENTS: AN EXAMINATION OF THE DATA THAT INFLUENCED RECENT UPDATES TO THE BEERS CRITERIA

\section{H Heith Durrence}

San Diego State University, San Diego, California, USA

AIM: Pain and insomnia are two common health-related complaints that are often comorbid and that increase in prevalence with age. The American Geriatrics Society updated the Beers Criteria for potentially inappropriate medication use in older adults in 2012. These changes clarified that doxepin (DXP) doses $\leq 6 \mathrm{mg}$ are considered appropriate for use in the elderly population. This report reviews data of DXP (Silenor) $3 \mathrm{mg}$ and $6 \mathrm{mg}$ in elderly insomnia patients that were the basis for these changes.

METHODS: Safety endpoints from two double-blind placebo-controlled trials of elderly insomnia patients are reported. Study A was a 12-week trial and Study B was a four-week trial. Safety endpoints assessed include adverse event (AE) reporting, next-day residual effects, and sleep architecture.

RESULTS: Overall, DXP 3 mg and 6 mg were well-tolerated in both studies, with no apparent dose-related effects compared with placebo (PBO). Rates of treatment-emergent AEs for DXP $3 \mathrm{mg}$ and $6 \mathrm{mg}$ were similar to $\mathrm{PBO}$ in both studies. In terms of next-day residual effects, there were no significant differences between PBO and DXP $3 \mathrm{mg}$ at any time point in Study A. There were no reports of complex sleep behaviors, memory impairment or cognitive disorder in any DXP-treated patient.

CONCLUSIONS: Data indicate that DXP $3 \mathrm{mg}$ and $6 \mathrm{mg}$ were highly efficacious and well-tolerated, with no evidence of REM suppression or anticholinergic effects. Most relevant for this patient population, DXP at these doses significantly improved endpoints associated with sleep maintenance and early morning awakenings without evidence of next-day residual effects.

\section{D}

\section{INFANT NEGATIVE FACIAL EXPRESSIONS OF PAIN FOLLOWING IMMUNIZATION PROCEDURES OVER THE FIRST YEAR OF LIFE}

Monica O'Neill $^{1}$, Sara Ahola Kohut ${ }^{2,3}$, Rebecca Pillai Riddell1,2,3, Harriet Oster ${ }^{4}$

${ }^{1}$ York University; ${ }^{2}$ The Hospital for Sick Children; ${ }^{3}$ University of Toronto, Toronto, Ontario; ${ }^{4}$ New York University, New York, New York, USA

AIM: To determine if the average time spent displaying five different negative facial expression categories over $1 \mathrm{~min}$ post-needle differs at two, four, six and 12 months of age.

METHODS: Video footage of infants' facial expressions over 1 min post needle was coded using the Facial Action Coding System for Infants and Young Children (BabyFACS; Oster, 2010). Five negative facial expressions categories were developed according to the most frequently co-occurring negative facial expressions identified.

RESULTS: The average time spent in each of the facial expression categories was calculated for four $15 \mathrm{~s}$ epochs over $1 \mathrm{~min}$ post-needle (ie, $0 \mathrm{~s}$ to $14 \mathrm{~s}$, $15 \mathrm{~s}$ to $29 \mathrm{~s}, 30 \mathrm{~s}$ to $44 \mathrm{~s}, 45 \mathrm{~s}$ to $59 \mathrm{~s}$ ) at each immunization appointment (two, four, six and 12 months). A set of factorial repeated-measures ANOVAs were performed to compare the amount of time that infants displayed each of the negative facial expression categories within four $15 \mathrm{~s}$ epochs over $1 \mathrm{~min}$ post needle at each immunization appointment. There was a significant interaction between the $15 \mathrm{~s}$ epochs and the mean amount of time that the negative facial expression categories were displayed at two months, $\mathrm{F}(6.18,612.21)=25.19, \mathrm{P}<0.001$, partial $\eta 2=0.20$, four months, $\mathrm{F}(5.53,547.42)=24.29, \mathrm{P}<0.001$, partial $\eta 2=0.20$, six months, $\mathrm{F}(6.14$, 
$607.72)=16.32, \mathrm{P}<0.001$, partial $\eta^{2}=0.14$, and 12 months of age, $\mathrm{F}(5.71$, 565.59) $=7.84, \mathrm{P}<0.001$, partial $\eta^{2}=0.07$.

CONCLUSIONS: Infant negative facial expressions are displayed for a significantly different amount of time within four $15 \mathrm{~s}$ epochs over $1 \mathrm{~min}$ post needle for each age group.

\section{E}

RELATIONSHIP BETWEEN PATIENT SATISFACTION AND TREATMENT OUTCOMES IN CHRONIC PAIN COMMUNITY CLINICS

Kim Rod

McMaster University, Hamilton, Ontario

AIM: Chronic pain remains a major health crisis. In Canada alone, one in three persons (33\%) lives with chronic pain. Multidisciplinary treatments for chronic pain are shown to be effective. The objective of this case study was to determine whether patient satisfaction affects treatment outcomes. METHODS: Patients enrolled in an eight-week multidisciplinary pain program in a community pain clinic. Patients completed questionnaires about pain, psychological and emotional status, and satisfaction with program. Select questions were asked before and after the program and were designed to include patients' evaluation of the program, level of change, pain and coping perceptions, and psychological/emotional adjustment. Scales ranged according to the question.

RESULTS: Patients $(n=62)$ completed questionnaires. More than onehalf $(52.54 \%)$ rated their overall level of satisfaction with the treatment program as very helpful. At the start of the program, $24 \%$ of patients rated their level of pain as severe compared to $1.67 \%$ at the end of the treatment. All of the patients who completed the questionnaires reported that they would recommend the program to friends.

CONCLUSIONS: The results of this survey suggest a relationship between patient satisfaction and treatment outcome. More than half $(52.54 \%)$ of patients felt the treatment program was very helpful, and all the patients reported that they would recommend the program to friends. The effect of patient satisfaction on treatment outcomes warrants further investigation by well organized studies.

\section{3:45 PM - 4:45 PM SYMPOSIA}

\section{0}

\section{MANAGING OPIOID-RELATED SIDE EFFECTS - EVOLVING STRATEGIES}

\section{Chair : Roman D Jovey}

CPM Centres for Pain Management, Mississauga, Ontario Speakers: Roman D Jovey, Michael Boivin

Chronic non-cancer pain (CNCP) constitutes a major public problem in Canada, affecting more people than heart disease, cancer and diabetes combined. When available, the 'best practice' treatment of CNCP is a multimodal, interdisciplinary approach involving physical, psychological, pharmacological and interventional approaches. In spite of recent concerns regarding misuse, opioid analgesics remain an important option for pharmacological treatment. Recognizing and effectively treating common adverse effects can help to optimize treatment outcomes.

This symposium will review the most common opioid-related side effects and provide practical treatment approaches to management. This will include a focus on the most common opioid side effect - opioid-induced constipation.

Learning Objectives:

1. Discuss the role of the pharmacist in managing the most common side effects of long-term opioid therapy as well as the importance of certain drug-drug interactions.

2. Summarize current concepts regarding the assessment and management of opioid-related sedation/cognitive impairment and hormonal disturbances.

3. Describe a stepped treatment approach to opioid-induced constipation and evolving treatment options

\section{$10 \mathrm{~A}$}

THE ROLE OF THE PHARMACIST IN MANAGING OPIOIDRELATED SIDE EFFECTS

\section{Michael Boivin}

CommPharm Consulting Inc, Barrie, Ontario

This presentation will discuss how the pharmacist can help the patient and the clinician to manage some of the common side effects of opioid therapy as well as highlight the importance of drug-drug interactions.

\section{B}

\section{COMMON OPIOID SIDE EFFECTS - NEW THOUGHTS ON OLD PROBLEMS}

Roman D Jovey

CPM Centres for Pain Management, Mississauga, Ontario

This presentation will update clinical approaches to some common opioidrelated adverse effects, such as sedation/cognitive impairment, hormonal disturbances and a stepped approach to opioid-induced constipation.

\section{Session 109: 4:45 PM - 5:45 PM}

\section{1}

\section{CREATING A "WAY FORWARD PLAN" TO DEVELOP A RETURN TO WORK BEST PRACTICE GUIDELINE FOR INJURED WORKERS WITH PAIN}

\section{Chair: Joel Katz}

Department of Psychology, York University, Department of Anesthesia and Pain Management, Toronto General Hospital and University of Toronto, Toronto, Ontario Speakers: Lynn Cooper, Lynn Shaw, Bill Chedore WORKSHOP OBJECTIVE:

The aim of this symposium is to lay the groundwork and rationale for establishing a best practice guideline for injured workers with pain. The present system for managing pain in injured workers is dysfunctional and fraught with problems. Evidence suggests that collaborative approaches comprised of multiple stakeholders and/or team members can support the transition of persons with chronic pain and work related injuries back to work. However current research indicates that these approaches are fraught with difficulties in that they are not based on a framework that informs transitions and processes to support an injured worker who has pain in learning to change, adapt, adjust and take control over decisions in managing their health and functioning (Shaw et al, 2009). For instance, these workers experience problems accessing procedural or relevant health information, difficulty in understanding roles and responsibilities of self and providers, difficulties with the loss of a worker role identity, decreased self-confidence in directing their rehabilitation, lack of knowledge about the long term consequences of injury, difficulties with disruptions in family life and financial strain on daily living and difficulties in processing information regarding newly exposed disabilities and conflicting return to work demands (Antao, Shaw, Ollson, Reen, Bossers \& Cooper, 2013; Shaw, MacDermid, Daraz and Chedore, 2012; Shaw MacDermid, Kothari et al, 2010; Shaw, MacAhonic, Lindsay and Brake, 2009; Shaw, Knot Lindsay et al 2009). Given that many of the return to work approaches do not offer injured workers a space, tools, knowledge and strategies for processing information or managing the realities of day-today living or working with an injury underscored by pain, opportunities to synthesize knowledge and develop a way forward plan for more effective transitions are needed. In order to move forward, we are proposing the establishment of a best practice guideline for injured workers with pain. This will be accomplished by drawing on information from three perspectives: i) the injured worker with pain supported by pain organizations, ii) injured workers organizations and labour and iii) current research. Symposium attendees will engage in a dialogue with panelists in sharing their perspectives on what is needed in establishing a best practice guideline. Attendees will be asked to note their preferred level of input in helping in designing, testing, and/or implementing a best practice guideline that promote a way forward for injured workers living and working with pain. 
Learning Objectives:

1. To promote knowledge exchange between symposium participants and speakers on the need for a best practice guideline to promote sustainable solutions for injured workers with pain.

2. To engage attendees in identifying key imperatives for developing sustainable human, social and services/resource capital to support the needs of injured workers with pain.

3. To engage attendees in identifying ways that they or their networks or stakeholders can contribute to developing, testing, and implementing a best practice guideline for injured workers.

\section{$11 \mathrm{~A}$}

PERSON WITH PAIN PERSPECTIVES A NECESSARY VOICE FOR CREATING A BEST PRACTICE GUIDELINE FOR INJURED WORKERS

Lynn Cooper

Canadian Pain Coalition; Canadian Injured Workers' Alliance, Kitchener, Ontario

Ms Lynn Cooper will present the rationale and reasons for developing a best practice guideline from the perspectives of persons with pain. Ms. Cooper will provide a voice for the person with pain who is an injured worker relating experiential data and the knowledge of the lived experiences of others with pain. Constraints and challenges from the personal, societal, workplace, community, and health provider perspectives will be presented to highlight the complexity of issues that must be addressed. Potential solutions to these challenges will be presented. It is essential that injured workers with pain actively participate in the development of a "way forward plan" to promote better access to services and social change within communities.

\section{B}

\section{RESEARCH SUPPORTS THE NEED TO DEVELOP A GUIDLINE TO ENHANCE WORK OUTCOMES FOR INJURED WORKERS}

\section{Lynn Shaw}

Occupational Science, School of Occupational Therapy, University of Western Ontario, London, Ontario

Dr Lynn Shaw will present from research knowledge that synthesizes the perspectives of persons with pain, the strategies to promote work transitions for persons with pain, and employers' perspectives on hiring persons with disabilities that is relevant to the need for a way forward plan and a best practice guideline. Insights from recent scoping, realist synthesis and qualitative meta-synthesis will be used to present the current research that can inform change and solutions for injured workers with pain. Conceptual models and strategies that are used to promote work transitions, reestablishment of worker identity and return to meaningful productive work occupations will be presented. Networks with researchers and knowledge synthesis from the social, health, medical, rehabilitation and economic literatures are needed to inform change and to work with stakeholders to promote use of solutions in interventions to achieve enhanced work outcomes for injured workers with pain.

\section{C}

\section{INJURED WORKERS ORGANIZATION RATIONALE FOR DEVELOPING A BEST PRACTICE GUIDELINE \\ Bill Chedore}

Canadian Injured Workers' Alliance, Canadian Pain Coalition, Iroquois, Ontario

Mr Bill Chedore will present the rationale and reasons for developing a best practice guideline from the perspectives of injured worker groups and labour representatives. Mr. Chedore will provide an historical and current account, based on available information, of the injustices and inequities injured workers with pain face in living and working with pain. Historical and statistical information on the injured worker experience will be used to present a rationale for the need for a best practice guide.

Knowledge from the realm of employment inequities - unemployment, underemployment, work disengagement, stigma, financial barriers, retraining injustices, structural/system and institutional barriers and challenges will be presented to highlight the complexity of issues that must be addressed. Potential solutions to these challenges will be presented. Injured worker groups have access to knowledge and expertise that is essential to informing a "way forward plan" to promote knowledge

\section{Session 110: 4:45 PM - 5:45 PM}

\section{2}

\section{WEATHERING THE PAIN OF CLINICAL PRACTICE GUIDELINE (CPG) DEVELOPMENT, DISSEMINATION AND IMPLEMENTATION: A PRACTICAL WORKSHOP}

\section{Chair: Michael McGillion}

Faculty of Health Sciences, McMaster University, Hamilton, Ontario Speakers: Anna Taddio, Michael McGillion, Gary Walco

WORKSHOP OBJECTIVE:

This practical workshop will address the feasibility and related challenges of adhering to established international clinical practice guideline (CPG) standards. It will also address the utility of these systems - and the effectiveness of teams who use them - to promote uptake of CPGs and impact patient care.

Learning Objectives:

1. Identify key issues in clinical practice guideline team composition, process, and implementation.

2. Discuss the feasibility and related challenges of adhering to internationallyaccepted clinical practice guideline standards.

3. Engage in critical debate about the impact of pain clinical practice guidelines on patient care, with a focus on common strategies for dissemination and uptake.

\section{A}

\section{THOU SHALT AGREE TO CPGS}

Anna Taddio

Leslie Dan Faculty of Pharmacy, University of Toronto, Toronto, Ontario

Health care providers and researchers are increasingly turning to clinical practice guidelines (CPGs) to assist them interpret the vast amount of scientific literature. CPGs incorporate available scientific evidence with values and preferences to make recommendations about how to manage specific conditions. CPGs have the potential to improve care received by patients by promoting interventions with proven benefit and discouraging ineffective or harmful interventions. This presentation will review adherence to the latest international standards set forth by the Appraisal of Guidelines for Research \& Evaluation (AGREE) Collaboration; optimal adherence to AGREE standards is now required by the Canadian Pain Society Board of Directors. In addition, this presentation will introduce GLIA (GuideLine Implementability Appraisal), an instrument that predicts potential challenges to effective implementation of CPGs. Together AGREE and GLIA aid in identifying defects in the development and implementation of CPGs so that they may be remedied prior to CPG implementation. Finally, the need to carry out knowledge translation research to demonstrate the impact of CPGs will be discussed. Examples from a CPG about vaccination pain management will be used to illustrate the potential for CPGs to positively impact on health care delivery. 


\section{B}

ALAS POOR CPGS DISSEMINATORS, WE KNOW YOUR PLIGHT WELL! LESSONS LEARNED FROM DESIGN AND IMPLEMENTATION OF AN INNOVATIVE, WEB-BASED MODEL FOR INTERNATIONAL-SCALE DISSEMINATION OF AGREE-COMPLIANT GUIDELINES

Michael McGillion

School of Nursing, Faculty of Health Sciences McMaster University, Hamilton, Ontario

Disseminating AGREE-compliant CPGs broadly while ensuring adequate end-user incentive for update of their practice recommendations is challenging. This presentation will review, in detail, the process and partnerships required for developing a multi-media, web-based resource centre, designed for international-scale dissemination of the Joint Canadian Cardiovascular Society - Canadian Pain Society Guidelines for the Management of Refractory Angina. Novel technologies which enable widespread dissemination will be reviewed including: a) Opt-In Email Blasts to over 180,000 end-users across cardiology, pain, and emergency medicine; b) Key Words Real Estate Acquisition, designed to automatically give primacy on Google to CPG-related topics discussed by resource centre end-users; c) Electronic Banner Advertisements (EBA), created for installation on all relevant Elsevier journal websites, creating over 550,000 impressions; d) Integrated Mass Social Media Strategies, including a 'turn-key' button, designed to facilitate ease of access to the CPGs recommendations. This review of resource centre planning, design architecture and related challenges will be followed by an interim report on our dissemination metrics, powered by Google Analytics ${ }^{\circledR}$. Key features reported will include total 1) Total and Partial Stream Views of video-based CPG recommendations; and 2) unique end-user tracking patterns facilitated by our Geo-Targeting System, which track end-user countries and respective regions. This presentation will conclude with aggregate-level reporting of technical issues related to various end-user browsers and operating systems, and how we are suing these data to inform our decisions about ongoing resource centre maintenance and dissemination of our AGREE Compliant CPGs.

\section{$12 \mathrm{C}$}

MUCH ADO ABOUT CPGS: IS IT ALL WORTH IT?

\section{Gary A Walco}

Seattle Children's Hospital, Anesthesiology, University of Washington School of Medicine, Seattle, Washington, USA

While there is sound rationale for developing consensus guidelines and CPGs for managing pain, and mounting considerable effort to disseminate and implement them, there remains little concrete evidence demonstrating their utility and impact on patient care. Audits carried out in institutions caring for children, for instance, consistently demonstrate sub-optimal pain practices despite the presence of numerous guidelines. This presentation will review some of the challenges to the implementation of evidence-based strategies for pain in a pediatric institutional context. These challenges include environmental barriers, practical issues, and individual factors. Suggestions for changing the status quo in CPGs implementation will be reviewed and debated among our Symposium presentation team.
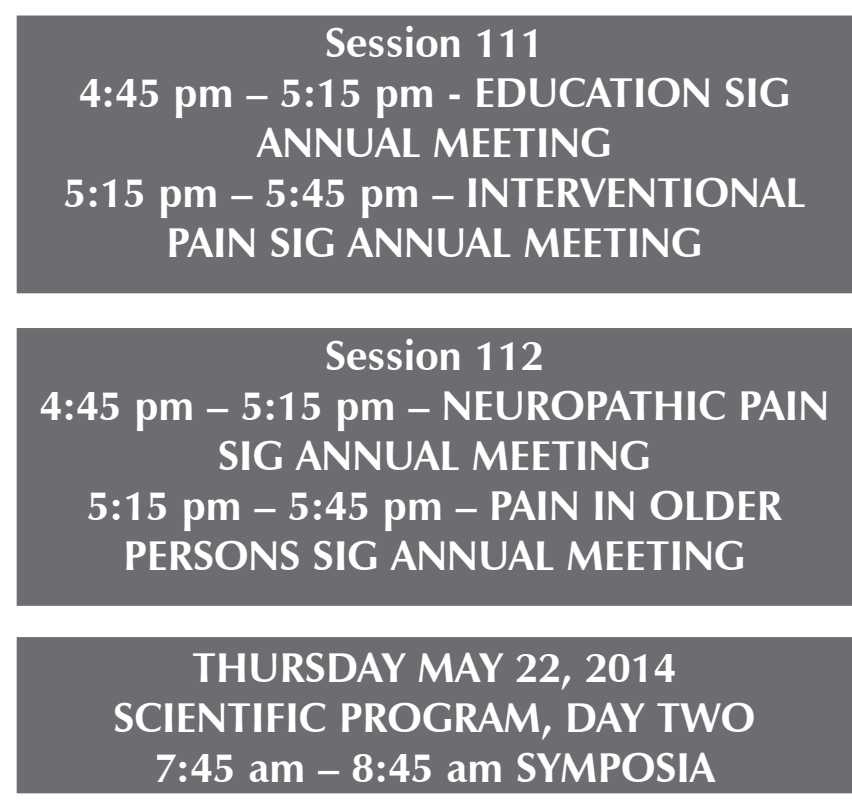

13

\section{PAIN RESEARCH: AMAZING OR JUST A MAZE? UNDERSTANDING AND APPLYING RESEARCH TO CLINICAL PAIN PRACTICE}

Chair: Roman D Jovey

CPM Centres for Pain Management, Mississauga, Ontario Speakers: Owen D Williamson ${ }^{1}$, Pam Squire ${ }^{2}$

${ }^{1}$ JPOCSC Pain Clinic, Surrey; ${ }^{2}$ University of British Columbia, Vancouver, British Columbia

For better or worse we practice in an era of evidence-based medicine. Clinicians are regularly admonished to follow evidence-based guidelines. However, what is the quality of evidence behind these recommendations? Who defines relevant outcomes? How can differences between groups inform decision-making for an individual patient? Some practical examples regarding the treatment of spinal pain will be presented to illustrate how clinicians can translate sometimes-confusing evidence into clinical practice.

Learning Objectives:

At the conclusion of this symposium, participants will be able to:

1. Describe the difference between statistically significant and clinically relevant changes in outcomes following pain interventions.

2. Describe the limitations of "gold standard" methodologies, such as randomized controlled trials and systematic reviews, in defining the safety and efficacy of pain interventions.

3. Define absolute and relative risk reduction and numbers needed to harm and describe the use and misuse of these numbers in comparing treatment options.

4. Identify alternate strategies for integrating the best available evidence, physician experience and patient preference in optimizing the treatment of individual patients with pain. 
KEYNOTE SPEAKERS 9:00 am - 10:00 am

\section{9:00 am - 9:30 am}

\section{4}

\section{TRANSLATIONAL PAIN RESEARCH: TARGETING CENTRAL} SENSITIZATION

Chair: Jeffrey S Mogil

EP Taylor Professor of Pain Studies, Canada Research Chair in the Genetics of Pain (Tier I), Department of Psychology and Alan Edwards Centre for Pain Research, McGill University, Montreal, Quebec

Speaker: Robert W Gereau, IV

Washington University Pain Center, Professor, Department of Anesthesiology, Washington University School of Medicine, St Louis, Missouri, USA

Central sensitization is a term given to the modulation of central neural circuits leading to the enhanced perception of pain. Central sensitization is evident in many chronic pain conditions, and can be clearly demonstrated in animal models and in patients. We and others have hypothesized that this abnormal central amplification is a major contributor to the development of chronic pain. This talk will describe work by our group and others that has identified metabotropic glutamate receptor 5 (mGlu5) as a key mediator of central sensitization in rodent model systems. We will then discuss ongoing studies aimed at testing whether a mGlu5 also mediates experimentallyinduced central sensitization in humans, and ultimately whether this mGlu5-dependent central sensitization is a mediator of central amplification pain in patients suffering from a variety of chronic pain conditions.

Learning Objectives:

To be aware of the key role of central sensitization in chronic pain

1. To understand the evidence for a role of metabotropic glutamate receptors in induction and maintenance of central sensitization

2. To understand the approaches being taken to translate preclinical findings first to healthy human volunteers and then to pain patients.

\section{9:30 am - 10:00 am}

\section{5}

\section{PAIN IN THE CRITICALLY ILL: A VOICE FOR THOSE WITHOUT WORDS}

\section{CPS EARLY CAREER AWARD RECIPIENT}

Chair: Christine T Chambers

Dalhousie University and IWK Health Centre, Departments of Pediatrics and Psychology and Centre for Pediatric Pain Research, Halifax, Nova Scotia

Speaker: Céline Gélinas

McGill University, Ingram School of Nursing, Montreal, Quebec

Dr Gélinas' research focuses on improving the assessment and management of pain in most vulnerable critically ill adult patients. Specifically, she has explored various pain assessment methods and created the CriticalCare Pain Observation Tool (CPOT) of which she is adapting the content for those suffering from a brain injury with altered level of consciousness. Her research also targets the development of innovative implementation strategies of an integrated systematic pain assessment approach in the intensive care unit (ICU) to optimize pain management practices and patient outcomes.

Learning Objectives

1. To examine current evidence on pain assessment in patients unable to self-report.

2. To describe pain-related behaviors in critically ill patients with or without brain injury and at different levels of consciousness.

3. To explore challenges and strategies associated with the implementation of pain assessment tools in the context of critical care.
Session 201: 10:45 am - 12:15 pm

16

\section{HOT TOPICS}

Chair: Bruce D Dick

Department of Anesthesiology and Pain Medicine, University of Alberta, Edmonton, Alberta

16A

BOYS VS GIRLS AND HOT VS COLD: A META-ANALYSIS OF SEX DIFFERENCES IN EXPERIMENTAL PAIN RESPONSES OF HEALTHY CHILDREN

Katelynn E Boerner ${ }^{1,2}$, Line Caes ${ }^{3}$, Kathryn A Birnie ${ }^{1,2}$,

Meghan G Schinkel ${ }^{1,2}$, Christine T Chambers ${ }^{1,2,4}$

${ }^{1}$ Department of Psychology \& Neuroscience, Dalhousie University;

${ }^{2}$ Centre for Pediatric Pain Research, IWK Health Centre; ${ }^{3}$ IWK

Health Centre; ${ }^{4}$ Pediatrics, Dalhousie University, Halifax, Nova Scotia

AIM: Sex differences in experimental pain are commonly reported in reviews of adult pain. The objective of the present research was to conduct a meta-analysis of sex differences in healthy children's experiences of experimental pain and, where possible, to conduct these analyses separately for children and adolescents, as previous research has suggested that developmental factors may be implicated in the emergence of sex differences.

METHODS: A search was conducted of electronic databases for published papers in English of empirical research using any experimental pain task to examine pain-related outcomes in healthy boys and girls between 0 and 18 years of age. Eligible studies were coded to extract information relevant to sex differences.

RESULTS: The search yielded 519 abstracts, with 80 separate studies eligible for inclusion. Data necessary for meta-analysis was available from 33 studies (1069 girls and 1040 boys). Meta-analyses revealed that girls reported significantly greater pain intensity than boys in cold pressor tasks studies when participants' mean age was greater than 12 years $(Z=2.35$; $\mathrm{P}=0.02$ ); boys had a greater heat pain threshold than girls in studies where participants' mean age was 12 years or younger $(\mathrm{Z}=2.46 ; \mathrm{P}=0.01)$; and boys had greater tolerance of heat pain than girls $(\mathrm{Z}=2.40 ; \mathrm{P}=0.02)$.

CONCLUSIONS: These findings suggest that sex differences in children are inconsistent across experimental pain methodologies, and that developmental stage may be relevant for understanding sex differences in pain.

\section{B}

NURSES' PERCEPTIONS OF MANAGING PAIN MEDICATIONS IN LONG TERM CARE

Sharon Kaasalainen, Gina Agarwal, Lisa Dolovich

McMaster University, Hamilton, Ontario

AIM: Nursing care in long-term care (LTC) homes is becoming more complex given the growing acuity of residents which coincides with more medications being administered to LTC residents. The purpose of this study was to explore LTC nurses' perceptions of their practices related to administering pain medications to LTC residents.

METHODS: A cross-sectional survey design was used that included both quantitative and open-ended questions. Data was collected from 165 licensed nurses (59\% response rate) at nine LTC homes in southern Ontario.

RESULTS: The majority (85\%) of all nurses felt that the medication administration system was adequate to help them manage residents' pain and $98 \%$ felt comfortable administering narcotics in LTC. In their decision to administer a narcotic to a LTC resident, nurses were influenced by pain assessments (ie, non-verbal and physical indications, verbal requests from residents or their family members); physician orders; diagnosis of resident (ie,, physical condition, resident was deemed palliative); past history; medical contraindications and allergies; effectiveness of non-narcotics; and fear of making dosage miscalculations or resident becoming addicted to 
narcotics. Finally, $63 \%$ of nurses reported being comfortable with giving analgesics to residents who were currently taking warfarin; most stating that they trusted the physicians and pharmacists to ensure that orders were safe and appropriate.

CONCLUSIONS: These study findings highlight the perceptions of nurses related to administering pain medications in LTC and related areas where continuing education initiatives are needed.

\section{C}

IMPAIRMENT OF CORTICOMOTOR CONTROL OF LUMBAR MULTIFIDUS IN CHRONIC LOW BACK PAIN

Hugo Massé-Alarie ${ }^{1}$, Louis-David Beaulieu' ${ }^{1}$, Richard Preuss ${ }^{2}$, Cyril Schneider ${ }^{3}$

${ }^{1}$ Centre de recherche du CHU de Québec, Université Laval, Laval;

${ }^{2}$ Centre de recherche Constance - CRIR, School of Physical and Occupational Therapy, McGill University, Montreal; ${ }^{3}$ Centre de recherche du CHU de Québec, Département de réadapation, Université Laval, Laval, Quebec

AIM: Chronic low back pain (CLBP) is often associated with impaired activation of deep trunk muscles. This may be linked to a maladaptive reorganization of the primary motor cortex (M1). Altered function of the lumbar multifidus (LMF) may be of particular importance, due to its clear role in maintaining spine stability. Our study aimed to examine the link between volitional activation of LMF and different parameters of M1 corticomotor control, using transcranial magnetic stimulation (TMS).

METHODS: Eleven individuals with CLBP and 13 healthy participants were tested with TMS of M1. The active motor threshold (AMT) and amplitude of motor evoked potentials of LMF, along with short-interval intracortical inhibition (SICI) and facilitation (SICF) - two mechanisms involved in motor programming - were assessed. Isometric contractions of LMF were also performed in prone lying, with the change in muscle thickness observed on ultrasound imaging used as a measure of volitional activation.

RESULTS: Two main differences were found between individuals with CLBP and healthy participants. First, the left M1 SICI was reduced in individuals with CLBP. Second, a correlation between M1 AMT and volitional activation of LMF that was observed in healthy participants was absent in individuals with CLBP.

CONCLUSIONS: These data provide evidence of altered M1 corticomotor control of the LMF in individuals with CLBP. Our main findings - a reduced SICI and the absence of a correlation between AMT and volitional activation - reflect changes in M1 function that may contribute directly to altered motor control and mechanical spine dysfunction in the CLBP population.

\section{D}

\section{PAIN, PSYCHOLOGICAL HEALTH AND EXECUTIVE FUNCTION IN A COLLEGE STUDENT SAMPLE}

Caitlin Murray ${ }^{1}$, Lorri Kais ${ }^{1}$, Tonya Palermo ${ }^{2}$, Grayson Holmbeck ${ }^{1}$, Rebecca Silton ${ }^{1}$

${ }^{1}$ Loyola University Chicago, Chicago, Illinois; ${ }^{2}$ Seattle Research Institute, Seattle, Washington, USA

AIM: Comorbid pain and depression lead to significant functional impairment. Pain and depression are known to influence specific brain regions that are associated with executive function (EF), yet little is known about their joint impact on EF. The primary aim of this study was to examine a model to test pain as a moderator of the relationship between psychological function and $\mathrm{EF}$ in an emerging adult population.

METHODS: Participants included 157 college students, 18 to 29 years of age $(66.2 \%$ female). Participants completed pain, EF, depression and worry questionnaires

RESULTS: One-half of the sample reported pain one to three times per week. Pain was reported to be of moderate intensity ( $M=4.04$; VAS 1-10). Regression analyses indicated that more frequent pain $(B=0.23, P<0.01)$ and greater symptoms of depression $(B=0.36, P<0.01)$ were associated with increased difficulties with inhibition. Greater symptoms of depression $(B=0.45, P<0.01)$ were associated with increased impairments in shifting attention. Pain frequency was a significant moderator of the relationship between psychological and EF. Greater symptoms of depression predicted greater difficulties with inhibition $(\mathrm{B}=-0.16, \mathrm{P}<0.05)$ and shifting $(B=-0.24, P<0.05)$, and this relationship was stronger in young adults who experienced frequent pain.

CONCLUSIONS: Pain and depression interact to negatively influence EF. Young adults with depression symptoms may be particularly vulnerable to develop EF impairments if they also experience frequent pain. Emerging adulthood represents a critical window of opportunity to optimize health behaviors to prevent future pain trajectories.

\section{$16 \mathrm{E}$ \\ VALIDATION OF THE PATIENT-REPORTED HAMILTON INVENTORY FOR COMPLEX REGIONAL PAIN SYNDROME}

Tara Packham, Joy C MacDermid

School of Rehabilitation Sciences, McMaster University, Hamilton, Ontario

AIM: The Patient-Reported Hamilton Inventory for Complex Regional Pain Syndrome (PR-HI-CRPS) is an outcome measure currently under development. There are currently no widely accepted self-report assessments specific to CRPS.

METHODS: Multi-faceted recruitment (flyers in pain centres, notices in a national CRPS patient support newsletter, and social network postings) was used to recruit 50 individuals with CRPS to participate in cognitive debriefing interviews. Readability of the scale and recommendations for additional scale items were judged by the participants as content experts. Interviews were recorded for qualitative analysis of emergent themes about the experience of living with CRPS.

RESULTS: Interm analysis $(\mathrm{n}=37)$ : The mean age of respondents was 48 years (range 15 to 81 years); M:F 33:4; mean duration of symptoms 69 months (four to 324 months); $89 \%$ had been working full-time before onset, but only $13.5 \%$ had returned full-time to their job. Items were highly correlated with Cronbach's alpha $=0.93$ for total scale and ranging from 0.72 to 0.87 for the subscales. Participants believed the questions gave a clear overall picture of their daily experience with CRPS but noted two key concepts (depression and temperature/colour changes) were underrepresented in the current iteration, and identified problematic wording in several items. Qualitative themes include pain as an invisible disability, the need for better CRPS education for both patients and health professionals, planning skills as essential for coping, and anxiety about the potential impact of their condition on their future health and function.

CONCLUSIONS: Preliminary results generally support the clarity and content of a proposed patient-reported assessment tool for CRPS. Further research is needed to examine the reliability and other forms of validity of the next iteration of the PR-HI-CRPS.

\section{$16 \mathrm{~F}$}

INDIVIDUALS WITH CHRONIC WHIPLASH DISPLAYING WIDESPREAD SENSORY HYPERSENSITIVITY SUCCESSFULLY RESPOND TO CERVICAL RADIOFREQUENCY NEUROTOMY Ashley Smith ${ }^{1}$, Gwendolen Jull ${ }^{1}$, Geoff Schneider ${ }^{2}$, Bevan Frizzell ${ }^{2}$, R Allen Hooper ${ }^{2}$, Michele Sterling ${ }^{3}$

${ }^{1}$ Division of Physiotherapy, NHMRC Centre of Clinical Excellence Spinal Pain, Injury and Health, University of Queensland, Brisbane, Australia; ${ }^{2}$ Department of Medicine, University of Calgary, Calgary, Alberta; ${ }^{3}$ Centre of National Research on Disability and

Rehabilitation Medicine, The University of Queensland, Brisbane, Australia

AIM: To determine if individuals with chronic whiplash symptoms displaying widespread sensory hypersensitivity (WSH) respond to cervical radiofrequency neurotomy (cRFN); and which clinical features predict success of RFN.

METHODS: This prospective cohort study investigated 53 individuals with chronic WAD (26 individuals with WSH; 27 without) who underwent cRFN. Measures included: quantitative sensory tests (pressure [PPT]; thermal pain thresholds; brachial plexus provocation test [BPPT]); nociceptive flexor reflex (NFR), and cervical range of movement. Self-report 
pain and disability measures were also collected. Questionnaires included the General Health Questionnaire (GHQ-28), Posttraumatic Stress Diagnostic Scale (PDS) and Pain Catastrophizing Scale (PCS).

RESULTS: Cervical RFN resulted in improvements in physical and psychological measures in both groups over time $(\mathrm{P}<0.0001)$, with similar duration of effect. Individuals without ' $\mathrm{WSH}$ ' demonstrated greater pain relief $(\mathrm{P}=0.016)$. The 'WSH' group demonstrated significant improvements pre/post-cRFN for all physical measures (except NFR), psychological distress and pain catastrophizing; however continued to demonstrate reduced PPT and BPPT post-cRFN in comparison to the non-WSH group. Low levels of pain catastrophizing predicted success of cRFN.

CONCLUSIONS: Individuals with chronic WAD respond to cRFN, (improving physical and psychological measures), irrespective of degree of sensitization present in their clinical features. Low levels of pain catastrophizing also predicted a successful response to RFN.

\section{G \\ PREVALENCE AND DETERMINANTS OF CANNABINOID PRESCRIPTION FOR THE MANAGEMENT OF CHRONIC NON-CANCER PAIN: A POSTAL SURVEY OF PHYSICIANS Huguette St-Amant $^{1}$, Mark Ware ${ }^{2}$, Nancy Julien ${ }^{1}$, Anaïs Lacasse ${ }^{1}$ ${ }^{1}$ Département des sciences de la santé, Université du Québec en Abitibi-Témiscamingue, Rouyn-Noranda; ${ }^{2}$ The Alan Edward Pain Management Unit, McGill University Health Centre, Montréal, Québec}

AIM: Measure prevalence and determinants of cannabinoid prescription for the management of chronic non-cancer pain (CNCP).

METHODS: In February 2013, a postal survey was sent to all physicians of the region of Abitibi-Témiscamingue, Quebec, using a modified Dillman method. Measures included the past-year prevalence of cannabinoid prescription for the management of $\mathrm{CNCP}$, characteristics of physicians' medical practice, and factors that could increase physicians' comfort level with cannabinoid prescribing. Univariate and multivariate logistic regression models were used to identify determinants of cannabinoids prescription.

RESULTS: The response rate was $52.2 \%$, for a total of 166 physicians (family physicians: $56 \%$ ). A majority of physicians (79.2\%) did not attend continuing medical education activities about cannabinoids in the past year. The prevalence of cannabinoid prescription for the management of CNCP was $23.0 \%(91.1 \%$ of these physicians prescribed cannabinoids to $\leq 5$ patients). Among prescribers, $92.1 \%$ reported having prescribed nabilone, $18.4 \%$ medical marijuana, $5.3 \%$ THC/cannabidiol, and 0\% dronabinol. Adjusting for physicians' years of practice, speciality, practice setting, caseload, and perceived $\mathrm{CNCP}$ prevalence, multivariate models showed that physicians' comfort level with cannabinoid prescribing for the management of CNCP (OR 1.25 [95\%CI 1.01 to 1.55]) was the only determinant of an increased likelihood of prescription. Prescribers and non-prescribers reported that most important factors that could increase their comfort level with cannabinoid prescribing for the management of $\mathrm{CNCP}$ were continuing medical education, guidelines/algorithms including cannabinoids, and clinical data/studies (reported by $\geq 50 \%$ of physicians).

CONCLUSIONS: Results suggest a low prevalence of cannabinoid prescribing for the management of $\mathrm{CNCP}$ and this study will help the planification of continuing medical education.

\section{$16 \mathrm{H}$ \\ THE EFFECT OF QUANTITATIVE SENSORY TESTING AND PATIENT FACTORS ON PAIN, FUNCTION AND HEALTH STATUS IN SHOULDER DISORDERS}

\section{Zakir Uddin, Joy C MacDermid, Jaydeep Moro}

McMaster University, Hamilton, Ontario

AIM: To estimate the extent to which quantitative sensory test (QST) and patient factors predict pain, function and health status in people with shoulder disorder. To determine if there are gender differences for QST measures in this patient population.

METHODS: The study protocol was approved by the Hamilton Integrated Research Ethics Board. Thirty-four surgical and 10 nonsurgical participants were recruited. Participants were asked to complete self outcome measures (pain, function and health status), and then underwent performance test and QST. Pearson's $r$ was computed to find out relationships between QST variables and patient factors with either pain, function or health status. Eight regression models were build to analysis QSTs and patient factors separately as predictors of either pain, function or health status. An independent sample t-test was done to evaluate the gender effect on QST.

RESULTS: Greater PP threshold and PP tolerance was significantly correlated with higher shoulder functional performance $(r=0.31$ to 0.44$)$ and lower self-reported shoulder disability $(\mathrm{r}=-0.32$ to -0.36$)$. VT and CPT were not significantly related to pain, function or health status, with the exception of VT on the little finger where lower threshold was correlated to a lower pain intensity $(\mathrm{r}=0.50)$ and better functional performance $(\mathrm{r}=-0.34)$. Higher comorbidity was consistently correlated $(\mathrm{r}=0.31$ to 0.46) with poorer pain, function and health status. Older age was correlated to more pain intensity and less function ( $r=0.31$ to 0.57$)$. In multivariate models, patient factors contributed significantly to pain, function or health status models ( $\mathrm{r}^{2}=0.19$ to 0.36 ); whereas QST did not. QST was significantly different between males and females (in PP threshold [3.9 to 6.2; $\mathrm{P}<0.001$ ] and PP tolerance [7.6 to $12.6 ; \mathrm{P}<0.001$ ] and $\mathrm{CPT}$ [1.6 to 2.3; $\mathrm{P}=0.02])$.

CONCLUSIONS: The study found a relationship between some QST measures and pain-function. QST failed to predict pain, function and health status; whereas patient factors (except gender) predicted pain or function and health status. Most of the QST measures demonstrated more sensitive to female.

\section{$16 I$}

PAIN IN EXTREMELY LOW BIRTH WEIGHT SURVIVORS: SEVERITY AND STABILITY FROM ADOLESCENCE TO ADULTHOOD

\ordana Waxman ${ }^{1}$, Ryan J Van Lieshout ${ }^{2}$, Saroj Saigal ${ }^{2}$, Michael H Boyle ${ }^{2}$, Louis A Schmidt ${ }^{2}$

${ }^{1}$ York University, Toronto; ${ }^{2}$ McMaster University, Hamilton, Ontario AIM: The objective of this study was to compare the level and stability of pain concerns among those born at extremely low birth weight (ELBW; $<1000 \mathrm{~g}$ ) and normal birth weight (NBW) from 12 to 16 years of age, to 30 to 35 years of age.

METHODS: Longitudinal methods were employed to follow 84 ELBW survivors born between 1977 and 1982, and 78 sociodemographically matched NBW controls. Participants were asked to rate pain concerns at 12 to 16 years of age (teen), 22 to 26 years of age (young adult), and 30 to 35 years of age (adult). A composite measure of physical pain was derived at each visit by summing five variables on the somatic scale of the Child Behaviour Checklist/Young Adult Self-Report. Items included, "aches and pains," "headaches," "nausea," "skin problems," and "stomach aches".

RESULTS: When using an ANOVA with repeated measures with a Greenhouse-Geisser correction, the mean scores for physical pain were statistically different across time, $\mathrm{F}(1.959,313.460)=3.967, \mathrm{P}=0.02$, but not between groups.

CONCLUSIONS: Individuals born at ELBW did not differ on a composite measure of physical pain concerns than their NBW controls. Overall, pain concerns decreased across the lifespan; however, there was a trend toward the ELBWs exhibiting an increase in pain at 30 to 35 years of age. This may reflect possible differences in physical pain that emerge as the ELBW survivors approach middle age. 
Session 202: 10:45 am - 12:15 pm

\section{7}

\section{SUCCESSES AND FAILURES IN THE IMPLEMENTATION OF BEST PRACTICES IN PAIN CARE}

Chair: Thomas Hadjistavropoulos

University of Regina, Centre on Aging and Health and Department of Psychology, Regina, Saskatchewan

Speakers: Mary E Lynch, Thomas Hadjistavropoulos, Jennifer Stinson

\section{WORKSHOP OBJECTIVE:}

Wide-scale implementation of best practices in pain assessment and management is lacking in many areas. The purpose of this symposium is to discuss successes and failures in the wide scale implementation of best practices and to identify barriers and facilitators of implementation success. The general public policy context will be considered.

Learning Objectives:

1. To familiarize participants with: a) the challenges of translating the science of pain into improved care for people living with it; b) major advocacy initiatives, including the outcomes of these initiatives, nationally and internationally; and c) the status of the Canadian Pain Strategy, including examples of successes in other jurisdictions.

2. To familiarize participants with: a) specific implementation attempts of pain assessment/management best practices in long-term care facilities; $b$ ) successes and failures of such implementation efforts; and c) recommendations to improve the odds of permanent best practice implementation in both long-term care and other settings (these recommendations are based both on past experiences with implementation efforts and on the scientific research).

3. To familiarize participants with: a) specific implementation attempts of pain assessment/management best practices in pediatrics; b) successes and failures of implementation efforts; and c) lessons learned and recommendations for ensuring implementation of best practices in the management of acute pain in children.

\section{A}

\section{GOALS, CURRENT OUTCOMES, AND FUTURE DIRECTIONS OF THE CANADIAN PAIN STRATEGY}

\section{Mary Lynch}

Pain Management Unit, Queen Elizabeth II Health Sciences Centre and Dalhousie University, Halifax, Nova Scotia

Chronic pain in Canada remains undertreated despite clear evidence that it has a greater negative impact on quality of life than other chronic conditions. Chronic pain also reduces ability to function with resultant major economic loss to individuals and to society. There is also strong evidence that acute pain is undertreated in emergency rooms and on postoperative hospital wards and that the situation has not improved over the past decade. Research, documenting both the problem and appropriate solutions for the implementation of cost effective treatments, has not addressed adequately the problem of undertreatment. Factors contributing to this situation and to the development of an international advocacy movement, supporting appropriate care of pain as a basic human right, will be discussed. Lessons learned from the Canadian Pain Strategy initiative along with an update regarding provincial successes will be presented. Critical movement forward in other nations, such as Australia, South America, and the US Military and contributors to progress in this area will be discussed.

\section{B}

MOVING PAIN ASSESSMENT FOR LONG-TERM CARE RESIDENTS WITH DEMENTIA INTO THE 21ST CENTURY: HAS ANYTHING CHANGED?

Thomas Hadjistavropoulos

Department of Psychology and Centre on Aging and Health, University of Regina, Regina, Saskatchewan

Effective pain assessment in older adults with dementia presents special challenges because cognitive decline impairs ability to express pain verbally. Consequently, in long-term care settings, pain problems often remaine unidentified, resulting in unnecessary suffering. Over the last 10 years there have been major advances in this area. That is, effective pain assessment approaches and related long-term care protocols have been developed. These methods not only help identify pain and fluctuations in the pain experience but their regular use leads to better pain management, more appropriate use of medication and reductions in staff stress. As documented in the peer reviewed literature, public policy experts and front-line health care workers have concluded that implementation of evidence-based pain assessment protocols in long-term care settings is both feasible and highly desirable. Yet best practices in this area have not been implemented on a wide scale, despite efforts to accomplish this goal. This presentation will include specific examples of pain assessment protocol implementation failure along with a systematic examination of the reasons and circumstances that led to unsuccessful implementation. Lessons learned and related recommendations will be included in the presentation.

\section{C}

\section{MOVING PAIN ASSESSMENT AND MANAGEMENT OF ACUTE PAIN IN CHILDREN INTO THE 21ST CENTURY: HAS ANYTHING CHANGED?}

\section{Jennifer Stinson}

Child Health Evaluative Sciences, Hospital for Sick Children and Lawrence S Bloomberg Faculty of Nursing, University of Toronto The field of pediatric pain has expanded immensely in the past two decades bringing forward considerable research progress. Despite this progress, the assessment and management of pain in children is often suboptimal. For example, in Canadian hospitals, children receive, on average, six painful procedures per day, most often with inadequate pain management. This occurs in spite of knowledge that unmanaged pain results in increased morbidity, mortality, and costs due to delayed healing and increased length of hospital stay. Untreated pain early in life affects brain development, is associated with health care avoidance, and negatively influences emotional and social functioning. Untreated acute pain can also lead to chronic pain. Furthermore, an increasing number of studies have focused on the way healthcare professionals manage children's pain. These studies indicate that practices do not always conform to current evidence-based best practice guidelines. This presentation includes discussion of an example of an innovative knowledge translation intervention that was successful in improving the assessment and management of acute pain in hospitalized children. A review of other efforts, that were not successful in improving clinical outcomes or in changing health care providers' attitudes/ behaviours, will also be presented. Lessons learned and related recommendations will be included in the presentation.

\section{Session 203: 10:45 am - 12:15 pm}

\section{8}

\section{INNOVATIONS IN CHRONIC PAIN AND ADDICTION: TELE-MENTORING THROUGH PROJECT ECHO ${ }^{\circledR}$ (EXTENDING COMMUNITY HEALTHCARE OUTCOMES) Chair: Ruth E Dubin}

Department of Family Medicine (adjunct), Queens University, Kingston, Ontario

Speakers: Jeannie Boyle, Daniel Duhigg, Ruth E Dubin WORKSHOP OBJECTIVE:

Overall aim of the symposium: To introduce participants to an innovative, validated, and replicable knowledge translation approach that results in positive practice change and increased system capacity.

Learning Objectives:

1. To understand how Project $E C H O \circledR$ differs from standard telemedicine

2. To recognize the benefits of the ECHO® model for chronic pain and addictions.

3. To consider how the model can improve primary care management and specialist access. 
18A

TELE-MENTORING MULTIPLIES PAIN EXPERTISE

Jeannie Boyle

The ECHO Institute, University of New Mexico, Albuquerque, New Mexico, USA

Project ECHO®, an interprofessional tele-mentoring program for health professionals, was developed at the University of New Mexico Health Sciences Center to fill a gap in specialty-care access for common, treatable conditions. Using both authentic cases and evidence-based didactics, primary care providers join a weekly video network to gain knowledge, skills and experience in managing conditions like addictions, hepatitis $\mathrm{C}$, rheumatology and chronic pain.

To measure the success of the Chronic Pain tele-ECHO clinic, three different approaches were used: 1) individual participant attendance and case presentations were aggregated; 2) continuing medical education (CME) evaluations were analyzed; and 3) practice change questions were asked in two focus groups of clinicians who participated for at least one year.

Between January 2010 through December 2012, 136 ECHO Pain clinics were held. There were 3835 total instances of participation, representing 753 unique individuals. $60 \%$ self-identified as advanced practice or other non-physician health professionals. More than $4500 \mathrm{CME}$ hours were issued and a total of 710 anonymous evaluations were assessed. Statistically significant improvements in participant self-reported knowledge, skills, and practice were demonstrated. Focus group analyses detailed specific practice improvements resulting from joining ECHO Pain.

There are substantial burdens on systems already plagued with too few primary care clinicians and specialists to meet current demand. The ECHO Pain model is a means to fill the considerable gap in pain expertise by implementing effective, evidence-based, work-based education and continuing professional development for health professionals.

18B

WHEN WORLDS COLLIDE: CO-STEWARDSHIP OF CHRONIC PAIN, ADDICTION, AND MENTAL HEALTH USING THE ECHO MODEL

Dan Duhigg

Department of Psychiatry, Department of Neurology, ECHO Institute, University of New Mexico, Albuquerque, New Mexico, USA

Project ECHO's Chronic Pain/Headache and Integrated Addictions/ Psychiatry tele-health clinics are a forum for primary care providers (PCPs) to present challenging cases to an inter-professional network of specialists. PCPs receive real-time feedback and recommendations on complex patients with unmet needs due to psychiatric, substance use, chronic pain, and/or disadvantageous social determinants of health. ECHO is a forum for the most difficult cases to be managed by the primary care provider with input from expert mentors in a supportive, accessible community of practice.

Project ECHO also provides training and certification for Suboxone use, as well as PCP education in evidence-based treatments for chronic pain, addiction and mental health issues including rational prescribing, nonpharmacological approaches, motivational interviewing, and harm reduction.

New Mexico has one of the highest rates of accidental opioid deaths in the United States, and the highest rate of alcohol-related mortality. It is largely rural and mostly medically underserved, with severely limited access to mental health, chronic pain and addiction services. Project ECHO eliminates the geographic divide by connecting primary care providers with specialists in order to deliver evidence-based care to patients where they live. The ECHO approach can mean that PCPs embrace these complex patients with confidence, and maintain the crucial supportive patientprimary care physician relationship.
$18 \mathrm{C}$

\section{HIGH HOPES: A CHRONIC PAIN/OPIOID STEWARDSHIP PROJECT ECHO IN ONTARIO?}

\section{Ruth E Dubin}

Department of Family Medicine (adjunct), Queens University, Kingston, Ontario; College of Family Physicians of Canada

New Mexico and Ontario share many barriers to quality care in chronic pain, addictions and mental health. These include: isolated and poorly trained primary care providers ( $\mathrm{PCPs}$ ), long distances, restricted access to specialty care, high rates of undertreated pain and opioid overdoses, and many socially disadvantaged ethno-cultural communities.

Project ECHO's mission includes replication and dissemination of complex chronic disease management programs both nationally and internationally. The ECHO University of New Mexico team follows the tenets of having each practitioner work at their highest proficiency level, increasing system capacity, leveraging scarce resources, rapid access to specialist mentors and disseminating evidenced-based, best practice knowledge. The ECHO originator, Dr. Sanjeev Arora operates from a public health perspective: our goal is to make the world a better place. Hence all ECHO content and methodologies are de-monopolized and shared freely with collaborators.

An Ontario-based inter-professional team assembled by Drs Dubin and Andrea Furlan attended the ECHO replication orientation session in Albuquerque (August 2013). As part of a wider chronic pain strategy we are working with the Ministry of Health of Ontario to deliver an ECHO chronic pain/opioid stewardship demonstration project to isolated PCP's, using existing telemedicine videoconferencing infrastructure.

Discussion of the replication process and early results from this initiative will be presented. If successful, an ECHO model may be highly appropriate in other Canadian provinces and applicable to other disease states.

\section{Session 204: 10:45 am - 12:15 pm}

\section{9}

\section{UPDATE ON BASIC HEADACHE MECHANISMS}

Chair: Brian Cairns

University of British Columbia, Vancouver, British Columbia

Speakers: Barry J Sessle, Dan Levy, Brian Cairns WORKSHOP OBJECTIVE:

Migraine headache is a very common pain condition, but the mechanisms underlying pain in this condition are still being unraveled. This session is intended to bring participants up to speed with current advances in our understanding of these mechanisms.

This topic will be of interest to research scientists interested in craniofacial pain mechanisms. This topic will also be of interest to clinicians who treat patients with migraine headache.

Learning Objectives:

Upon completion of this session, attendees will learn about:

1. The role of non-neuronal cells in trigeminal pain mechanisms.

2. The neural processes thought to initiate migraine headaches.

3. How botulinum neurotoxin may act to decrease chronic migraine headaches.

\section{A}

TRIGEMINAL PAIN MECHANISMS: THE ROLE OF NON-NEURAL CELLS

Barry Sessle

Faculties of Dentistry and Medicine, University of Toronto, Toronto, Ontario

Several acute and chronic pain conditions in the face or mouth are very common, and some are unique to the orofacial region. However, the etiology and pathogenesis of most orofacial chronic pain conditions are unresolved, and they are difficult to diagnose and manage. This talk will provide an overview of the neural mechanisms underlying orofacial pain with a focus on recent findings indicating that nonneural cells, specifically satellite cells in the sensory ganglia and astroglia and microglia cells in the central nervous system, are important players in both acute and chronic 
inflammatory and neuropathic orofacial pain conditions and may offer new targets for management of these conditions.

\section{B}

\section{MECHANISMS OF HEADACHE PAIN INITIATION}

\section{Dan Levy}

Harvard Medical School, Boston, Massachusetts, USA

Migraine is considered one of the most prevalent neurological disorders but its underlying pathophysiology is poorly understood. Over the past two decades, it became widely accepted that activation of primary afferent nociceptive neurons that innervate the intracranial meninges serves as a key process that mediates the throbbing head pain of migraine. Knowledge about the endogenous factors that play a role in promoting this neural process during a migraine attack slowly begins to increase, and a better understanding remains one of the holy grails in migraine research. This talk will review key experimental data implicating complex meningeal immuno-vascular interactions, in particular, the interplay between proinflammatory cytokines, the meningeal vasculature and immune cells, in enhancing the responses of meningeal nociceptors.

\section{C}

\section{HOW DOES BOTULINUM NEUROTOXIN WORK FOR HEADACHE?}

Brian Cairns

University of British Columbia, Vancouver, British Columbia

The mechanism by which intramuscular injection of BoNTA into the craniofacial muscles decreases migraine headaches is not known. Research suggests that injection of BoNTA into craniofacial muscles may have a fairly rapid analgesic action through a direct effect on muscle nociceptor excitability as well as an indirect effect on neurogenic inflammation. This talk will review evidence and current theories related to the mechanism of action of BoNTA when used to treat chronic headache.

\section{Session 205: 1:30 pm - 3:00 pm TRAINEE SESSION}

\section{0}

\section{BEYOND LIFE AS A PAIN RESEARCH TRAINEE: NAVIGATING WORKFORCE ENTRY AND MAINTAINING A HEALTHY WORK-LIFE BALANCE}

Chair: Whitney Scott

Clinical Psychology, McGill University, Montreal, Quebec

Speakers: Timothy H Wideman, Michael Hildebrand,

\section{Christine T Chambers}

A career in pain research can be extremely rewarding. Exploring your job options and planning accordingly may increase your chances of success. The aim of this trainee-oriented symposium is to provide clinical and basic science trainees with guidance for pursuing careers in pain research after graduate school. Presenters will highlight opportunities and challenges in the transition from graduate school to establishing a successful career in pain research. The symposium will also discuss insights and strategies for balancing a career in pain research with a healthy personal life. Active participation among attendees will be encouraged.

Learning Objectives:

1. Identify opportunities and discuss challenges in the transition from pain research in graduate school to successfully obtaining a job in academia.

2. Identify opportunities and challenges for establishing careers in clinical and basic science pain research.

3. Discuss strategies for successfully balancing a career in pain research with a healthy personal life.

\section{A}

HOW TO GET LUCKY AFTER GRAD SCHOOL AND OTHER CAREER ADVICE TO PAIN RESEARCH TRAINEES

Timothy $\mathbf{H}$ Wideman

Pain Science Division, Canadian Physiotherapy Association; Johns Hopkins University, Baltimore, Maryland, USA

Thomas Jefferson once wrote, "I am a great believer in luck, and I find the harder I work the more I have of it". Finding a career that is both enjoyable and meaningful requires luck and a lot of hard work. This presentation will reflect on the presence of these two elements throughout the presenter's training experiences and how they might be relevant for current trainees. The speaker will discuss generating, implementing, and sharing ideas as essential elements of research training. A theoretical model that relates these elements to the trainee, the environment, and luck will be proposed, drawing on experiences from the presenter's own training. Participants will be asked to reflect on whether their experiences are consistent with this model and whether they can generate their own personal models for success as pain researchers.

\section{B}

THE GOOD, THE BAD, AND THE UGLY: LESSONS LEARNED DURING THE PURSUIT OF A CAREER IN BASIC SCIENCE PAIN RESEARCH

Michael E Hildebrand

Department of Neuroscience, Carleton University, Ottawa, Ontario The pursuit of a career in academic research can be both very challenging and very rewarding, at both personal and professional levels. Hindsight is always 20:20, so this presentation will draw on the presenter's personal experiences during a professional and geographical journey from academia to industry and back to academia, with three children being born along the way. Based on these experiences, general principles and strategies will be presented for navigating an academic career path.

\section{C}

DATA, DEADLINES, DIAPERS AND DINNER: ONE PAIN RESEARCHER'S TAKE ON LIFE BALANCE

Christine T Chambers

Dalhousie University and IWK Health Centre, Halifax, Nova Scotia Trainees embarking on careers in pain research and practice often wonder how they will be able to balance both their professional and personal goals. This presentation will provide suggestions for trainees on how to achieve life balance and how to manage both 'work' (career and ambition) and 'lifestyle' (health, pleasure, leisure, family) goals. Stories will be shared from the perspective of a clinical pain researcher who is also a busy and proud mom of four young children.

\section{Session 206: 1:30 pm - 3:00 pm}

\section{1}

\section{THE CHALLENGES OF PEDIATRIC PAIN: WHAT IS THE ROLE OF BIOMARKERS?}

Chair: Catherine E Ferland

McGill University; Shriners Hospital for Children - Canada; Alan

Edwards Centre for Research on Pain, Montreal, Quebec

Speakers: Catherine E Ferland, Ruth E Grunau, Frank Symons

WORKSHOP OBJECTIVE:

Pain among children is a serious public health problem. There are a number of related clinical reasons for the development and application of biobehavioral assessment technologies built around a biomarker approach to assess pain in high-risk children (neonates born prematurely, children with cognitive and communicative impairments, children undergoing surgery). Such an approach would: 1) better inform a primary health care provider's diagnosis; 2) provide guidance toward rational treatment strategies; 3) provide a benchmark from which to evaluate treatments; and 4) assist the identification of pain correlates thereby providing more 
information about the occurrence of pain and the setting in which it occurs. Integrating biological (eg, heart rate, stress hormone, inflammatory or nociceptive biochemicals) and behavioral (eg, facial actions) signs or markers of pain are an intuitively appealing idea but a daunting scientific and clinical research challenge.

With these general objectives in mind, this symposium is built around three research groups representing on-going interdisciplinary, translational research programs developed to identify biophysical, biochemical and neuroanatomical changes associated with chronic pain in children and their predictive validity for pain etiology, severity and functional impairment. Because of the elusive nature of pediatric pain, particularly in preverbal or nonverbal children, therapeutic decisions are frequently based on trial and error, a situation frustrating to both physician and patient (and their families). To improve therapeutic outcomes and quality of life for sufferers of chronic pain associated with developmental disorders or children at risk for chronic pain problems because of early experience, there is an urgent clinical need to develop advanced diagnostic tools based on an expanded understanding of the mechanisms underlying chronic pain associated with early (aberrant) noxious experience or myriad factors associated with neurodevelopmental disorders.

The goal of this symposium is to describe biomarkers that may allow us to better understand and treat pediatric pain. To accomplish this goal, the symposium will describe complementary programs of research conducted in subclasses of the pediatric population with their specific challenges: neonates, children with developmental delays and adolescents.

Learning Objectives:

1. Identify the various pain outcomes within different age groups and developmental needs

2. Describe what is known about objective assessment of pediatric pain in the clinical world

3. Explain biomarkers of nociception and their impact in the clinical environment

\section{A}

\section{VALIDATION OF BIOMARKERS TO OPTIMIZE THE POST- OPERATIVE PAIN MANAGEMENT IN ADOLESCENTS UNDERGOING SPINAL SURGERY}

\section{Catherine E Ferland}

McGill University; Shriners Hospital for Children - Canada; Alan Edwards Centre for Research on Pain, Montreal, Quebec

Pro-nociceptive and pro-inflammatory mediators sensitize sensory fibres, leading to central changes in the dorsal horn of the spinal cord that alter the transmission and modulation of the afferent signal. Clinically important factors such as sensitivity or insensitivity to certain treatments could be reflected by a unique biochemical profile in the cerebrospinal fluid (CSF). Studying CSF allows the assessment of markers of abnormal central nervous system activity. However, its collection is invasive and impractical. Knowing that many biomarkers may eventually equilibrate in the blood, those encountered in the CSF may also be found in the blood and eventually in saliva. If blood and saliva co-vary with CSF, we would then negate the need to collect CSF in future studies. The validation of biomarkers could lead to an optimization of the post-operative pain management. Investigation of biomarkers simultaneously in CSF, blood and saliva in adolescents undergoing spinal surgery will be presented, and their relationship with validated self-reported pain perception pre- and post-surgery will be discussed.

\section{B}

\section{CHALLENGES OF PAIN INDICATORS IN PRETERM INFANTS Ruth E Grunau}

Developmental Neurosciences \& Child Health, Child \& Family Research Institute; Pediatrics, University of British Columbia, Vancouver, British Columbia

Infants are unable to express their pain using self-report. Therefore pain assessment relies on indirect or surrogate indicators, including pain behaviours such as facial actions, crying and body movements, that are typically considered concurrently with physiologic changes including increased heart rate, higher cortisol and lower oxygen saturation. Biomarkers and behaviours are modestly correlated, which is important but challenging in interpretation of pain. Developmental considerations are essential for assessing pain of very preterm infants who are hospitalized during a critical period of very rapid brain development. Currently, the use of measures of brain activity including the recording of electrical activity along the scalp indexed by electroencephalography (EEG, aEEG) and changes in cerebral hemodynamics and oxygenation detected by near infrared spectroscopy (NIRS) are of great interest in neonatal pain, however their contribution and meaning is unclear as yet in the understanding of pain. The role of biomarkers needs to be considered in the differing context of procedural and persistent pain.

\section{C}

\section{PAIN IN CHILDREN WITH NEURODEVELOPMENTAL DISABILITY - THE PROMISE OF BIOMARKERS}

\section{Frank Symons}

Department of Educational Psychology, Centre for Neurobehavioral Development, University of Minnesota, Minneapolis, Minnesota,

\section{USA}

The mechanisms that explain why some children with neurodevelopmental disorders (eg, severe intellectual disabilities, autism, etc.) experience analgesic failure after exposure to optimal pain treatment remain unclear. Predicting who will and who will not respond to a given analgesic regimen currently relies on clinical trial and error. This clinical problem is compounded further by the fact that children with neurodevelopmental disorders frequently have altered cognitive and communicative capacities making it difficult to distinguish pain from other stress/arousal. The problem is significant as children with neurodevelopmental disabilities experience markedly higher rates of pain (30\% to $50 \%)$ than their typically developing peers with enormous associated costs (extended hospital stays, emotional cost of suffering, reduced adaptive function). A major advance in pain assessment and treatment in this context could be made by translating basic research evaluating associations between pain etiologies and biomarker profiles to complement clinical assessments. To date, no work has systematically examined the validity of central and peripheral neuroendocrine biomarkers, their relationship to noxious (ie, painful) events and pain-related behaviors in children with neurodevelopmental disorders. Ultimately, such biomarkers should have predictive potential to improve outcomes and increase our understanding of basic mechanisms underlying analgesic response. Investigation of biomarkers simultaneously in CSF and saliva in children with cerebral palsy undergoing surgery for intrathecal pump implants will be presented, and their relationship proxy-reported pain perception pre- and post-surgery will be discussed.

\section{Session 207: 1:30 pm - 3:00 pm}

\section{2}

\section{THE CHANGING LANDSCAPE OF PRESCRIPTION OPIOID} ABUSE AND MISUSE: A NORTH AMERICAN EPIDEMIC

\section{Chair: Megan J Shram}

Altreos Research Partners Inc, Department of Pharmacology and

Toxicology, University of Toronto, Toronto, Ontario

Speakers: Megan J Shram, Richard Dart, Lorella Garofalo

WORKSHOP OBJECTIVE:

To provide an overview of recent data monitoring prescription opioid abuse in Canada and to provide insights to the regulatory and clinical approaches at mitigating risks

Learning Objectives:

1. Provide an overview of changing regulations in Canada and the United States in addressing prescription opioid abuse.

2. Discuss available Canadian data, methodological challenges and future efforts in assessing the magnitude of prescription opioid abuse.

3. Present various risk mitigation strategies ranging from the patient to the federal level and follow with an open-forum discussion of these topics. 


\section{A}

MITIGATING PRESCRIPTION OPIOID MISUSE AND ABUSE: REGULATORY INITIATIVES IN CANADA AND THE UNITED STATES

Megan Shram

Altreos Research Partners, Inc; Department of Pharmacology and Toxicology, University of Toronto, Toronto, Ontario

The misuse, abuse and diversion (MAD) of prescription opioids is a significant public health problem in North America. To address this crisis, several regulatory strategies have been or are being proposed for implementation. Recent changes to address prescription opioid MAD will be presented and discussed, including guidance for sponsors developing abuse-deterrent formulations (ADFs), post-marketing surveillance approaches and requirements, and prescription monitoring programs; challenges in the development of ADFs will be highlighted. The impact of generic availability and requirements for abuse-deterrent properties on patient access to opioid medications will also be described in the context of societal costs, such as drug abuse treatment, medical treatment of overdoses, and lost productivity. The presentation will be followed by a discussion on how to achieve an appropriate balance between risks and benefits in continuing to provide patients with adequate pain relief, while still achieving meaningful and important reductions in prescription opioid MAD.

\section{B}

\section{THE CHANGING LANDSCAPE OF PRESCRIPTION OPIOID} ABUSE AND MISUSE: A NORTH AMERICAN EPIDEMIC

\section{Richard Dart}

Rocky Mountain Poison and Drug Center;RADARS ${ }^{\circledR}$ System,

Denver Health; Emergency Medicine, University of Colorado,

Denver, Colorado, USA

Canada is the world's second largest per capita consumer of prescription opioids (NACPDM, First Do No Harm). Multiple studies have indicated that retail availability of a drug is directly related to its rate of abuse. Some First Nations in Canada have declared a community crisis owing to prescription drugs (Dell et al., 2012). One implication of the recommendations in First Do No Harm is the need for post-marketing surveillance of prescription opioids. Abuse and diversion data from three new systems will be presented to quantitate endorsement of abuse by Canadian respondents as well as street sales and price of prescription opioids in Canada. Finally an objective evaluation of media activity on the prescription opioids in Canada will be provided.

One important focus involves the introduction of abuse deterrent formulations in Canada and the United States. The initial results of this natural experiment will be evaluated by presenting new data on the abuse and diversion of oxycodone in Canada. Before 2010, the original, crushable formulation of oxycodone ER (OxyContin $\left.{ }^{\circledR}\right)$ was available in both countries. In 2010, the original crushable formulation of OxyContin was replaced in the US by a crush resistant formulation. The same formulation, named OxyNeo, was introduced in Canada in 2011/2012. However, the original crushable formulation of oxycodone ER has remained available in Canada through generic pharmaceutical companies. Initial analysis of the impact of the persistence of the original formulation will be presented.

\section{C}

\section{IDENTIFYING AND MITIGATING THE RISK OF PRESCRIPTION OPIOID ABUSE, MISUSE AND DIVERSION IN PAIN PATIENTS AND THE UNINTENDED USER}

\section{Lorella Garofalo}

\section{Pfizer Canada Inc}

Prescription opioid abuse continues to be a growing concern across Canada. Mitigation strategies to address the problem have included additional surveillance systems to monitor abuse as well as guidelines for the safe and effective use of opioids for chronic non-cancer pain. Recent Canadian data suggests that harm from nonmedical prescription opioid analgesic use has increased in past years. Data from limited studies in recreational opioid users have also indicated that drug diversion is a predominant source of obtaining prescription opioids for nonmedical use, a trend that has also been observed in the United States. Recent studies have also shown that aberrant drug-related behaviors have been reported in $40 \%$ to $50 \%$ of pain patients and that approximately one in 10 patients reported to have willingly or unwillingly engaged in prescription opioid diversion. Studies evaluating risk mitigation strategies have shown a tendency for physician's to underestimate the potential risk of patients for prescription opioid misuse, abuse, and diversion (MAD). Current risk assessment tools for clinical practice do not adequately risk of diversion and most do not evaluate motivations of behaviors that allow physician's to differentiate between abuse or misuse. An overview of available clinical risk assessment tools and clinical approaches to structured opioid therapy will presented and discussed. A discussion will be convened to assess how to enhance risk mitigation strategies to address prescription opioid MAD and how such strategies can be employed at the patient, prescriber and payer level.

Session 208: 1:30 pm - 3:00 pm

\section{3}

\section{SYMCHRONIC POSTSURGICAL PAIN: UTILIZING KNOWN PREDICTORS TO DEVELOP PREVENTIVE TREATMENT STRATEGIES}

Chair: Joel Katz

Department of Anesthesia \& Pain Management, Toronto General Hospital; Department of Anesthesia, University of Toronto; Department of Psychology, York University, Toronto, Ontario Speakers: Joel Katz, Michael Andreae, Hance Clarke WORKSHOP OBJECTIVE:

The practice of treating pain only after it has become well entrenched is slowly being supplanted by a preventive approach that aims to prevent peripheral and central sensitization brought about by incision and subsequent noxious perioperative factors. The transition of acute postoperative pain to chronic post surgical pain is a complex and poorly understood process involving biological, psychological and social-environmental factors. Dr Joel Katz and will review some of the risk and protective factors for the development of chronic postsurgical pain and will describe a biopsychosocial approach to CPSP prevention. Dr Michael Andreae will contrast the concepts of preventive versus preemptive analgesia, he will discuss perioperative regimens that in randomized trials demonstrated a reduction in the transition from an acute perioperative pain to a chronic postsurgical pain syndrome. Finally, Dr Hance Clarke will present rates and risk factors for prolonged postoperative opioid use in opioid naïve patients undergoing major surgery. Using a case based format, he will also discuss perioperative management strategies that might reduce the development of chronic postsurgical pain syndromes. A discussion of the implications and directions for future research follows the presentations.

Learning Objectives:

1. To discuss the risk and protective factors for the development of chronic postsurgical pain and present a biopsychosocial framework for prevention.

2. To present recent findings that suggest the ability to prevent/ decrease the incidence of chronic postsurgical pain.

3. To present recent findings which present rates and risk factors for prolonged postoperative opioid use in opioid naïve patients undergoing major elective surgery.

\section{A \\ BIOPSYCHOSOCIAL PREDICTORS OF CHRONIC POSTSURGICAL PAIN \\ Joel Katz}

Department of Anesthesia \& Pain Management, Toronto General Hospital; Department of Anesthesia, University of Toronto; Department of Psychology, York University, Toronto, Ontario Chronic postsurgical pain (CPSP) develops in an alarming proportion of patients. Dr Katz will review the epidemiology of chronic postsurgical pain. Risk and protective factors that predict the transition of acute pain to 
CPSP will then be presented, highlighting surgical, psychosocial, socioenvironmental, and patient-related factors that appear to confer a greater or lesser risk of developing chronic postsurgical pain. The rationale for a biopsychosocial approach to prevention will be presented emphasizing what can be done from a psychosocial perspective to minimize CPSP. Although we know more and more about the factors that facilitate and protect against the development of CPSP, the transition of acute postoperative pain to CPSP remains a complex and poorly understood developmental process involving biological, psychological and social-environmental factors.

\section{B}

\section{PREVENTIVE PERIOPERATIVE ANALGESIC STRATEGIES}

\section{Michael Andreae}

Montefiore Medical Center, Albert Einstein College of Medicine of Yeshiva University, Bronx; Mount Sinai Medical Center, Icahn School of Medicine, New York, New York, USA

Chronic postsurgical pain after surgery (CPSP) is a devastating condition with few treatment options. Dr. Andreae will summarize the evidence for local anesthetics and regional anesthesia for the prevention of chronic postsurgical pain. The effective nociceptive blockade afforded by local and regional anesthesia may inhibit the central sensitization that leads to hyperalgesia and CPSP. This presentation will focus on the many surgical interventions in which regional anesthesia techniques have shown to reduce the risk of CPSP. Dr. Andreae has recently published a meta-analysis of several RCTs which suggests that epidural anesthesia reduces the risk of persistent post- thoracotomy pain and that para-vertebral block prevents CPSP after breast surgery. Furthermore, the presenter will explain the systematic review process and address the limitations of his conclusions Other perioperative regimens from the literature that have demonstrated promise with respect to decreasing the transition from an acute pain to a chronic postsurgical pain will also be presented.

\section{C}

PUTTING THE PREDICTORS INTO PRACTICE: PREVENTIVE TREATMENT STRATEGIES

\section{Hance Clarke}

Department of Anesthesia \& Pain Management, Toronto General Hospital; Department of Anesthesia, Sunnybrook Health Sciences Centre; Department of Anesthesia, University of Toronto, Toronto, Ontario

Measuring pain intensity and analgesic use is not sufficient as outcomes for perioperative trials aimed at predicting/modifying the development of chronic postsurgical pain (CPSP). Proper evaluation and management of risk factors for CPSP require inter-professional teams to characterize a patient's experience of postoperative pain and to examine pain arising during functional activities. The development of CPSP may be related to the persistent use of opioid medications following surgery. This presentation will present novel information regarding rates and risk factors for prolonged postoperative opioid use in opioid naïve patients undergoing major surgery. Finally, a case based discussion of preventive treatment strategies will integrate information provided by the two previous presentations and stimulate discussion with respect to utilizing known predictors to decrease the development of CPSP in complex medical patients undergoing major surgery.
Session 209: 4:00 pm - 5:30 pm

\section{4}

\section{EARLY CHILDHOOD NONPHARMACOLOGICAL PAIN MANAGEMENT: A SYNTHESIS OF CURRENT RESEARCH AND CHALLENGES}

Chair: Rebecca Pillai Riddell

Department of Psychology, York University; Hospital for Sick Children; University of Toronto, Toronto, Ontario Speakers: Rebecca Pillai Riddell, Marsha Campbell-Yeo, Anna Taddio WORKSHOP OBJECTIVE:

Recent large scale reviews and studies document widespread unmanaged early childhood pain across (eg, Pillai Riddell et al, 2013; Taddio et al, 2010; Johnston et al, 2011) both primary and tertiary settings. The inability for these children to self-report pain, the difficulty with conducting analgesic and anesthetic trials for proper labeling in young children, and the move towards empowering parents in healthcare has resulted in a burgeoning interest innon-pharmacological approaches to early childhood pain management. An evolving paradigm shift embracing non-traditional approaches to pain management has precipitated a rapidly growing research body in using non-pharmacological strategies in medical settings. This sessioin sets out to review the current state of research in this area. Learning Objectives:

1. Update audience knowledge base of empirically supported nonpharmacological pain management strategies in infancy and early childhood via recent meta-analyses.

2. Discuss exemplars for both small-scale and large scale knowledge mobilization efforts.

3. Address challenges for future research and barriers for knowledge mobilization efforts outside established research contexts.

\section{A}

\section{EMPIRICALLY SUPPORTED STRATEGIES FOR PRIMARY CARE AND THE MYTH OF THE AVERAGE INFANT PAIN RESPONSE}

Rebecca Pillai Riddell

Department of Psychology, York University; The Hospital for Sick Children; University of Toronto, Toronto, Ontario

The first talk by Pillai Riddell will review the current state of the evidence in regards to non-pharmacological methods of pain management for infants and young children that occur in during the care of healthy infants and young children (eg, immunization). She will present research on evidence from an updated 2014 Cochrane Review. Moreover, based on recent work (Pillai Riddell et al, 2013), she will also discuss a conceptual conundrum regarding the use of averaged or mean pain responses as an outcome in early childhood Randomized Controlled Trials for pain management that needs to be addressed to move our field forward.

\section{B}

\section{MANAGING PAIN ON THE NEONATAL INTENSIVE CARE} UNIT: SUCCESSES AND CHALLENGES

Marsha Campbell-Yeo

Faculty of Health Professions, School of Nursing; Department of Pediatrics \& Centre for Pediatric Pain Research, Dalhousie University; IWK Health Centre, Halifax, Nova Scotia

Campbell-Yeo will provide state of the art evidence on nonpharmacological pain management techniques used within the hospital environment, specifically the neonatal intensive care and postbirth units. Concentrated focus will be placed on reviewing evidence for kangaroo care, co-bedding, breastfeeding and environmental modification based on recently published research (eg, Campbell-Yeo et al, 2013). Challenges related to knowledge uptake within the hospital environment will be addressed (eg, McNair, Campbell-Yeo et al, 2013) and results from recent work related to novel methods to facilitate practice change targeting both care providers and parents will be discussed. 
24C

IT TAKES A VILLAGE: CHANGING PRACTICE AT A INDIVIDUAL AND POLICY LEVEL

Anna Taddio

Leslie Dan Faculty of Pharmacy, University of Toronto, Hospital for Sick Children, Toronto, Ontario

Through the HelpINKids network, Taddio has led a multidisciplinary team of stakeholders through a successful guideline development project. This guideline for pediatric immunization pain management has been given accolades by a number of international and national organizations (Taddio et al, 2010) and is currently in the process of active knowledge mobilization. This presentation will review novel strategies in trying to mobilize and disseminate a guideline for practice change in pediatric pain management. Strategies in creating innovative mediums for pain management (eg, prenatal education workshops modules, parent training videos, and YouTube video clips) will be reviewed and team challenges shared. Taddio will also discuss barriers faced in building a knowledge mobilization network in terms of team development, team engagement, and dissemination challenges with clinicians and parents

\section{Session 210: 4:00 pm - 5:30 pm}

\section{5}

\section{DOES SEX MATTER? SEX $\times$ GENE INTERACTIONS IN HUMAN PAIN}

Chair: Luda Diatchenko

McGill University, Department of Anesthesia, Faculty of Medicine,

Faculty of Dentistry, Montreal, Quebec

Speakers: Luda Diatchenko, Inna Belfer, Jeffrey S Mogil WORKSHOP OBJECTIVE:

The future of personalized pain medicine will be based on individual difference factors contributing to variability in pain and analgesic responses, such as sex and genetic polymorphisms. Moreover, it is increasingly appreciated that these factors interact with each other to impact pain and analgesia. Sex differences in pain have been extensively documented, however the particular interactions between sex and genes regarding pain are still not fully uncovered. This session will provide current research insights on a wide spectrum of fundamental differences in the genetic contributions to variability in pain and analgesic responses within each sex, and mechanisms underlying these differences. A particular emphasis will be placed on the implications of the findings for clinical practice.

Learning Objectives:

1. Describe recent findings regarding sex-specific genetic influences on pain responses.

2. Enumerate particular genetic markers that are associated with pain and analgesia in a sex-specific manner.

3. Identify the importance of these findings for pain treatment and for clinical trial design.

\section{A}

\section{COMT SEX-SPECIFIC EFFECTS ON PAIN IN MICE AND \\ HUMANS}

\section{Luda Diatchenko}

\section{McGill University, Montreal, Quebec}

Dr Diatchenko will present recent findings from several mouse studies and human cohorts regarding contributions of the catechol-O-methyltransferase (COMT) gene to pain phenotypes. COMT is an enzyme that metabolizes catecholamine neurotransmitters and is a critical contributor to pain perception. Both human and mouse COMT genes possess functional polymorphisms contributing to interindividual variability in pain phenotypes. Dr Diatchenko will discuss how COMT genetic variations contributed to pain behaviors in mice and pain ratings in humans in a modality-specific and sex-specific manner.

\section{B}

SEX $\times$ GENE INTERACTIONS PREDICTING HUMAN PAIN

Inna Belfer

University of Pittsburgh, Pittsburgh, Pennsylvania, USA

Dr Belfer will describe and discuss the findings on sex $\times$ gene interactions from human association studies of acute and chronic post-surgical pain conditions, as well as the practical relevance of these findings.

\section{C}

\section{AVRP1 POLYMORPHISMS AND SEX IN CAPSAICIN PAIN AND VASOPRESSIN ANALGESIA}

\section{Jeffrey $S$ Mogil}

McGill University, Montreal, Quebec

Dr Mogil will review evidence from animal and translational pain research demonstrating how sex interacts with genes to influence analgesic responses. Future directions will be discussed.

\section{Session 211: 4:00 pm - 5:30 pm}

\section{6}

\section{PAIN MANAGEMENT IN PENAL INSTITUTIONS}

Chair: Ellen $\mathrm{N}$ Thompson

University of Ottawa (retired), CPM Ottawa, Ottawa, Ontario

Speakers: Ellen N Thompson, Eric Wasylenko, Ruth Dubin,

Jeannie Boyle

WORKSHOP OBJECTIVE:

To mount an interactive workshop with input from a number of stakeholders, and discussion amongst attendees, working towards the goal of creating guidelines for best practices regarding pain management of prison inmates. Learning Objectives:

1. To understand the special challenges of treating pain in incarcerated individuals.

2. To understand the ethical implications for health care in general, and pain management, in particular, in prison inmates.

3. To explore possible solutions to managing pain in prisons, including i) Implementing withdrawal protocols for inmates previously prescribed opioids. ii) Explore the use of tamper resistant or abuse deterrent opioids to continue in prison pain management. iii) Explore the use of prison peer education pain self management programs.

\section{A}

SPECIAL ISSUES REGARDING PAIN MANAGEMENT IN JAILS Ellen $\mathbf{N}$ Thompson

University of Ottawa (retired); CPM Clinic, Ottawa, Ontario

Treating chronic pain remains a challenge, in large part due to documented lack of education in medical school curriculae (Watt-Watson 2007). When individuals on long term opioid treatment are taken into custody this represents very difficult issues for the individual and for prison medical staff. Abrupt discontinuation of medications will precipitate abrupt withdrawal. This will not be life threatening, except for in medically 'fragile' individuals. It should be avoided if at all possible. To what extent do human rights issues apply? Can incarcerated individuals demand continuation of ongoing medical therapy? Are some drugs (insulin, antibiotics, antihypertensives) normally dispensed? Are there classes of drugs regularly excluded? While opioids remain the most problematic drug class in prison settings, other types of drugs are increasingly being abused. Practices and policies regarding medication dispensing vary between provincial and federal institutions. What policies rules and constraints apply to prison physicians? What is the role of provincial regulatory bodies, the Colleges of Physicians and Surgeons in ensuring good medical practices? The negative effect of sleep deprivation on pain, and possible solutions, will be discussed. Negative emotions, fear, anxiety, depression and catastrophizing also negatively impact the suffering from pain, and will need to be addressed. Distinguishing genuine pain patients from drug seekers or abusers is an extremely difficult problem in any setting, but particularly so in a detention or correctional facility. 
Input regarding these issues from Senior Medical Advsior to the Ministry of Public Safety and Corrections in Ontario, Dr Lori Kiefer, and from Dr Ruth Martin, Lead, Prison Medicine Committee, CFPC will be presented.

\section{B}

\section{ETHICS CONSIDERATIONS REGARDING INMATE PAIN MANAGEMENT}

Eric Wasylenko

Division of Palliative Medicine, Department of Oncology;

Department of Family Medicine, University of Calgary, Calgary;

John Dossetor Health Ethics Centre, University of Alberta,

Edmonton, Alberta; Joint Centre for Bioethics, University of

Toronto, Toronto, Ontario

Pain management for incarcerated individuals, whether in prisons, remand centres, forensic health assessment units or during episodic encounters in regular health facilities, is a challenge for clinical teams.

Normal ethical imperatives apply, including duties to care, confidentiality, non-maleficence, beneficence, respect for personhood, equivalency and least intrusiveness. But these are tempered by societal and individualfocussed notions of safety, duality, and security. Important issues of coercion, vulnerability, lack of care accessibility, lack of choice of provider, risk of diversion and prior addictions also influence the fulfilment of fiduciary physician-patient commitments.

Lack of national standards, general challenges in assessing pain and addiction - potentially heightened in the incarcerated population, and understandable conflicting commitments of providers place clinicians at risk of moral uncertainty. The result can be generation of disabling moral distress for clinicians, inconsistent practices and potential harm to incarcerated patients. Other countries and professional societies have begun to address these issues, publishing consensus guidelines that apply to their clinical, legal and regulatory environments.

Promulgation of Canadian standards, and provision of expert tools for best practices accounting for the unique clinical circumstances in caring for incarcerated patients, will be required. This work should involve at minimum, prison clinicians, acute, chronic and palliative pain experts, pharmacists, addiction specialists, ethicists and regulatory bodies. Ethics decision-making frameworks for general use, as well as the development of local ethics deliberative capacity for challenging circumstances should ideally supplement these standards and tools.

\section{C}

\section{PROJECT ECHO, THE PRISON PEER EDUCATION PROJECT}

Jeannie Boyle

The ECHO Institute; University of New Mexico Health Science

\section{Center, New Mexico, USA}

Pain and addictions management in correctional facilities can be difficult for a variety of reasons. Best pain and addictions practice recommends multimodal approaches from interdisciplinary teams to offer the most comprehensive means of care. Such approaches may be more challenging for this population.

Standards of practice for correctional health, pain and addictions exist but are not well defined:

The CDC has standards of practice materials dedicated to Correctional Health: http://www.cdc.gov/correctionalhealth/default.htm

Clinicians serving the correctional population in New Mexico cite need for more clinical knowledge related to pain and addictions.

What is Project ECHO® and what has it accomplished? :

Project ECHO® (Extension for Community Health Outcomes) has a history of success in training primary clinicians in the management of complex conditions using multipoint video teleconferencing and case consultation, together with measurement of outcomes for patients and clinicians (published in New England Journal of Medicine, 2011, Health Affairs, 201

Project ECHO® uses a Hub/Spoke design for the weekly TeleECHO ${ }^{\mathrm{TM}}$ clinics. Clinics usually convene during the noon hour and last $60 \mathrm{~min}$ to $120 \mathrm{~min}$. Surveys of participating clinicians cite the noon hour as the time most conducive to learning in a distraction free environment between morning and afternoon appointments.

Specialists at the "Hub" (University of New Mexico, Project ECHO®) communicate via telehealth technology with clinicians at "Spoke" sites in both urban and rural communities to present cases for consultation and to receive rich didactic education. The didactics often include interactive demonstrations such as 'How to do a focused examination' and 'How to utilize motivational interviewing to engage patient participation in self-management'.

\section{Session 212: 4:00 pm - 5:30 pm}

\section{7}

\section{TRACKING PAIN THROUGH TIME: FROM METHODOLOGY TO PRACTICE}

\section{Chair: Joel Katz}

Department of Psychology, York University, Department of Anesthesia and Pain Management, Toronto General Hospital and University of Toronto, Toronto, Ontario Speakers: M Gabrielle Pagé, Manon Choinière, Saifee Rashiq WORKSHOP OBJECTIVE:

Pain is dynamic and its inter-individual and intra-individual characteristics frequently change over time. The variability in pain within and across all stages, including sub-acute, acute and chronic, makes it difficult to predict, prevent and treat. The aim of this workshop is to explore some of the methodological and clinical challenges associated with pain variability over time. More specifically, the workshop aims to examine measurement and analysis issues with pain longitudinal data as well as to explore the clinical implications of pain variability.

Learning Objectives:

1. To understand methodological challenges associated with longitudinal data analysis and to learn various strategies to best model pain through time.

2. To illustrate with "real-life" data how the study of pain trajectories can help in understanding the clinical evolution of patients treated in tertiary care pain clinics.

3. To learn about the clinical challenges and implications of pain measurement in the broader context of chronic pain and disability.

\section{A}

\section{PAIN VARIABILITY OVER TIME: METHODOLOGICAL CHALLENGES AND STRATEGIES}

\section{Gabrielle Pagé}

Department of Psychology, Faculty of Health, York University, Toronto, Ontario

Research has consistently shown methodological issues associated with static pain measurement for both research and clinical practices. A limited number of studies have started to take into account intra-individual pain variability in the examination of the relationship between pain and outcomes of interest. This presentation will provide an overview of the advantages and limitations of various methodological approaches to pain variability and offer concrete recommendations to implement these measurement and statistical approaches. More specifically, this presentation has the following objectives: (1) to learn about the impact of pain variability on treatment responsiveness; (2) to understand the implications of measuring and ignoring pain variability in research outcomes; and (3) to learn about recent statistical developments in longitudinal data analysis.

\section{B}

\section{PAIN TRAJECTORIES IN PATIENTS TREATED IN TERTIARY CARE PAIN CLINICS}

\section{Manon Choinière}

Département d'anesthésiologie, Faculté de Médecine, Université de Montréal; Centre de recherche du Centre hospitalier de I'Université de Montréal (CRHUM), Montreal, Quebec

Results obtained from the Quebec Pain Registry in a large sample of patients attending multidisciplinary tertiary care pain clinics suggest significant but small improvement on various outcome measures six months after their initial visit. Furthermore, the observed improvement (or the absence of) at six months stayed the same 12 and 24 months later. These data were recently 
re-analyzed using growth mixture modeling techniques in order: 1) to identify homogeneous subgroups of patients based on their characteristic pain trajectories; and 2) to examine the relationships between these pain trajectories and health outcomes. Compared to conventional analytic practices, this approach is believed to provide better insight into the evolution of the clinical condition of chronic pain patients.

\section{C}

\section{IF I GET ANY BETTER, I'LL PROBABLY FEEL WORSE: THE CHALLENGES OF CHARTING THE PROGRESS OF CHRONIC PAIN OUTSIDE OF THE RESEARCH CONTEXT}

\section{Saifee Rashiq}

Department of Anesthesiology and Pain Medicine, University of Alberta, Edmonton, Alberta

Decreasing pain intensity, improving mood and reintegrating into life at home and at work ought to go hand in hand. So why is it that they so often do not? Does it matter if pain intensity goes down but function doesn't change? Or vice-versa? Come and bathe in the confusion and anguish as a clinical chronic pain therapist describes his Quixotic struggle to figure out if he is, in fact, doing his patients any good at all. Join the discussion about what markers of overall patient status in chronic pain we can defend as being valid to ourselves, to resource allocators and most importantly, to our patients. The objectives of this presentation are as follows: 1) to understand the potential for divergence between measures of pain and broader outcome measures in chronic pain and chronic pain syndrome; 2) o understand how the divergence between disease-specific and general outcome measures is handled in other chronic diseases; and 3) to learn about a range of clinically applicable outcome tools that might give useful information to the pain therapist.

\section{FRIDAY MAY 23, 2014
SCIENTIFIC PROGRAM, DAY THREE}

\section{8:00 am - 9:00 am SYMPOSIA}

\section{8}

\section{THE ENDOCANNABINOID SYSTEM AND THE EVIDENCE SUPPORTING A ROLE FOR CANNABINOIDS IN THE MANAGEMENT OF PAIN \\ Chair: Mary E Lynch}

Director of Research Pain Management Unit and Professor of Anesthesia, Pain Medicine and Perioperative Care, Psychiatry and Pharmacology, Dalhousie University, Halifax, Nova Scotia Speakers: Cecilia J Hillard, Andrea G Hohmann

The human body is equipped with a sophisticated endocannabinoid system that is involved in immune and pain defense as well as other activities (eg, stress buffering, anti-epileptic, bone metabolism, gut motility, spasticity). This session will start with an overview of the endocannabinoid system and the potential for exploiting this system in the treatment of human suffering, the possibility of an endocannabinoid deficiency syndrome in human pathology for example in depression and anxiety will also be presented. The second part of the symposium will focus on the therapeutic potential of cannabinoids and modulators of the endocannabinoid system in the treatment of chronic pain, including pathological pain produced by chemotherapeutic treatment. The growing literature examining the endocannabinoid system's role in modulation of pain and opportunity to exploit this system in the treatment of pain will be presented.

Learning Objectives:

Participants will have an appreciation of the components, mechanisms and actions of the endocannabinoid system.

At the end of this session participants will:

1. Be aware of evidence examining the endocannabinoid deficiency hypothesis.

2. Understand the role of cannabinoids in endogenous pain modulation.

3. Appreciate the potential for cannabinoids in treatment of chronic pain.

\section{9:15 am - 10:15 am}

\section{KEYNOTE SPEAKERS}

\section{9:15 am - 9:45 am}

\section{9}

\section{SMALL-FIBER POLYNEUROPATHY - BIG PLAYER IN CHRONIC PAIN?}

Chair: Jeffrey S Mogil

EP Taylor Professor of Pain Studies, Canada Research Chair in the Genetics of Pain (Tier I), Department of Psychology and Alan Edwards Centre for Pain Research, McGill University, Montreal, Quebec

Speaker: Anne Louise Oaklander

Syndromes involving unexplained chronic widespread pain such as fibromyalgia, chronic myalgic encephalitis, and seronegative Lyme are common and often a huge financial burden. When the patients are children, entire families can be disabled as parents leave work to care for children too sick for school. No pathological or pathophysiological causes had been discovered, leaving the presumption that the causes were psychological or ill-defined "central sensitization". Multiple labs are now reporting new pathological and physiological evidence that at least half of children and adults with these syndromes have SFPN. Diagnosing this when it is present offers the possibility of disease modifying treatment and new hope for patients and their families.

Learning Objectives:

1. To review the types of pain and other symptoms that suggest a patient might have SFPN.

2. To review the recommended diagnostic tests and treatment options for SFPN

3. To review new findings that SFPN is common in fibromyalgia and similar unexplained pain syndromes.

\section{9:45 am - 10:15 am}

\section{0}

HUSH HUSH, SWEET CHARLOTTE: REFLECTIONS ON THE CHALLENGES AND OPPORTUNITIES FOR DECREASING PAIN AND ENHANCING OUTCOMES IN INFANTS AND YOUNG CHILDREN

\section{CPS DISTINGUISHED CAREER AWARD RECIPIENT}

Chair: Christine T Chambers

Dalhousie University and IWK Health Centre, Departments of Pediatrics and Psychology and Centre for Pediatric Pain Research, Halifax, Nova Scotia

Speaker: Bonnie Stevens

Over the past few decades we have witnessed incredible growth in knowledge and expertise in preventing and managing pain in infants and young children. Although multiple and varied strategies are often supported by high quality research evidence, these are frequently not routinely implemented. Therefore, children continue to suffer from often preventable pain on a global basis. The consequences of this pain, especially in sick and hospitalized young children, are far reaching. This presentation will provide a critique of pain measurement approaches and management strategies to prevent and relieve pain in this vulnerable population and the challenges encountered in effectively implementing these solutions in everyday practice and life. This presentation will feature the research of $\mathrm{Dr}$ Stevens, her colleagues, and students over the past 20 years.

Learning Objectives:

After attending this session, participants will be able to:

1. Appreciate the nature and challenge of pain assessment in infants and young children in a changing landscape of novel opportunities and technologies. 
2. Understand the process of critical appraisal of pain management strategies and their immediate and long term impact on child health outcomes.

3. Distinguish between the challenges and opportunities for knowledge generation and knowledge translation across the spectrum of child global health.

\section{Session 301: 10:45 am - 12:15 pm}

\section{1}

\section{UNRAVELING THE IMPACT OF EARLY NEONATAL PAIN EXPOSURE - CHALLENGES IN TRANSLATION}

Chair: Marsha Campbell-Yeo

School of Nursing, Dalhousie University, Department of Pediatrics and Centre for Pediatric Pain Research, IWK Health Centre, Halifax, Nova Scotia

Speakers: Ruth E Grunau, Marsha Campbell-Yeo, Simon Beggs WORKSHOP OBJECTIVE:

Exposure to repetitive early pain and stress is inherit in the life of an infant born preterm and has been reported to be associated with altered brain development and thereby poorer neurodevelopmental outcomes. However, challenges remain as much of the research to date has overlooked various other clinical stressors found in modern neonatal intensive care units. These include hypoxemia, nutritional dysregulation, maternal separation, light, sound, sleep deprivation, the inflammatory conditions leading to preterm delivery and the inflammation brought on by modern therapies such as mechanical ventilation and antibiotics. We propose a symposium to discuss the difficulties encountered in previous research and propose a new paradigm in translational models. We will discuss methods to account for these various factors and how future research could be advanced. Our goal is to actively engage clinicians with pain neuroscientists to address how experimental conditions can be manipulated to study pain effects and management with more clinically relevant conditions.

Learning Objectives:

1. Describe the current knowledge of the impact of procedural pain in very preterm neonates hospitalized in intensive care.

2. Discuss the impact of adverse environmental and social context and possible links to pain-related outcomes.

3. Differentiate the overlooked factors influencing brain and neurodevelopment that may mask effects of neonatal pain and will be able to identify novel designs animal models and clinical studies to differentiate specific effects neonatal pain.

\section{$31 \mathrm{~A}$}

\section{PAIN, BRAIN AND NEURODEVELOPMENT IN PRETERM} NEONATES

\section{Ruth Grunau}

Department of Pediatrics, University of British Columbia;

\section{Developmental Neurosciences \& Child Health, Child \& Family}

Research Institute, Vancouver, Bristish Columbia

Very preterm neonates are vulnerable to repeated procedural pain at a time of rapid brain development and programming of stress systems. Current evidence suggests that repeated procedural pain contributes to long-term changes in stress regulation and brain development in these vulnerable children, after accounting for clinical confounders associated with prematurity. Complex bidirectional influences between the immune system, the central nervous system, and the hypothalamic-pituitary-adrenal (HPA) axis have received little attention in the context of effects of neonatal pain. Genetic variants that regulate inflammatory mechanisms appear to mediate the relationship between exposure to neonatal pain and altered axis function (cumulative cortisol levels) at age seven years, and may be sex specific. Evidence for the importance of multiple clinical factors in effects of early pain will be presented.

\section{$31 \mathrm{~B}$ \\ IMPACT OF SOCIAL CONTEXT AND ENVIROMENT ON NEONATAL PAIN RESPONSE}

Marsha Campbell-Yeo

School of Nursing, Dalhousie University, Department of Pediatrics

and Centre for Pediatric Pain Research, IWK Health Centre, Halifax, Nova Scotia

Pain, maternal separation, and adverse environmental context are inherent in the early life of infants born very preterm requiring prolonged hospitalization in the neonatal intensive care unit. Yet few studies examining long-term consequences of neonatal pain exposure have taken potential interactions between these factors into consideration. While it is known that maternal-centered interventions, such as skin-to-skin contact, diminish immediate pain reactivity and improves biobehavioral regulation, the potential neuroprotective influence is unknown.

\section{$31 \mathrm{C}$}

\section{MODELING EARLY PAIN EXPERIENCE - BRIDGING THE GAP}

Simon Beggs

The Hospital for Sick Children and University of Toronto, Toronto, Ontario

Animal research on neonatal pain typically assesses the effects of pain in the absence of other clinical factors known to affect long term neurodevelopment in infants born very preterm. Efforts are needed in experimental animal pain studies to model clinical features of prematurity. Differentiating effects of pain per se, pain treatment, versus other clinical factors is a major challenge. The extent to which factors such as prenatal and postnatal inflammation, hypotension and respiratory compromise can be addressed will be considered. Dialogue between bench scientists and clinicians is proposed in order to address advantages and limitations in animal models, and consider novel models to move forward the field of neonatal pain in this vulnerable population.

\section{Session 302: 10:45 am - 12:15 pm}

\section{2}

\section{RECENT ADVANCES IN UNDERSTANDING AND MANAGEMENT OF OROFACIAL PAIN \\ Chair: Barry J Sessle}

Faculties of Dentistry and Medicine, University of Toronto, Toronto, Ontario

Speakers: Barry J Sessle, Gilles Lavigne, Luda Diatchenko WORKSHOP OBJECTIVE:

There is a rapidly growing interest in orofacial pain, exemplified by this topic being the focus of the current IASP Global Year. Indeed, the face and mouth have special biological, emotional and psychological meaning to humans and most other animals, and represent sites of some of the most common pain states. The effects of chronic orofacial pain can be particularly problematic since it can be associated with emotional, psychological and social disturbances that can compromise the patient's quality of life and well-being, and can present a diagnostic and management challenge to clinicians. Nonetheless, several advances in our understanding of orofacial pain have been obtained over the past two decades from studies in laboratory animals and humans. This Symposium will address many of these advances, with new evidence-based approaches developed for the diagnosis and management of orofacial pain states and new evidence and insights into the biological, molecular and genetic processes underlying acute and chronic orofacial pain.

Dr Barry Sessle, who will chair the Symposium, will review the new insights into orofacial pain mechanisms that have been gained from recent studies using animal models of acute or chronic orofacial pain, Dr Gilles Lavigne will highlight the probable and debated relationship between orofacial-temporomandibular pain and breathing disorders or bruxism (tooth grinding) during sleep, and Dr Luda Diatchenko will review new findings bearing on genetic and phenotypic markers in orofacial pain conditions such as Temporomandibular Disorders. 
Learning Objectives:

1. Provide an improved understanding of the processes underlying orofacial pain and its control

2. Enhance understanding of the debated issues of the relationship between orofacial-temporomandibular pain and breathing disorders or bruxism during sleep

3. Improve understanding of the phenotypic and genetic signatures of TMD

\section{A}

\section{NEW INSIGHTS INTO MECHANISMS THAT MODULATE TRIGEMINAL CENTRAL SENSITIZATION IN NEUROPATHIC AND INFLAMMATORY OROFACIAL PAIN MODELS}

\section{Barry J Sessle}

Faculties of Dentistry and Medicine, University of Toronto, Toronto, Ontario

This presentation will review recent findings of processes that modulate trigeminal central sensitization following trigeminal nerve injury or inflammation and that relate to clinical features of orofacial neuropathic pain states and their management. Rodent models of trigeminal neuropathic or inflammatory pain may display mechanical and thermal hypersensitivities within or adjacent to the site innervated by the injured nerve as well as extraterritorially in other body sites innervated by other trigeminal nerves or by spinal nerves. These nociceptive behavioural changes reflecting allodynia, hyperalgesia and extraterratorial spread are accompanied by enhanced glutamate release in trigeminal subnucleus caudalis $\left(\mathrm{V}_{\mathrm{c}}\right.$, the 'medullary dorsal horn') and an increased excitability of Vc nociceptive neurons. This central sensitization appears to be a fundamental facilitatory process in the development and maintenance of chronic orofacial pain states manifesting allodynia, hyperalgesia and pain spread. The expressions of nociceptive behaviour and trigeminal central sensitization is modulated by several influences operating through a number of chemical mediators and receptor mechanisms (eg, NMDA, purinergic, GABA), and is also dependent on the functional integrity of non-neural cells (eg, Vc astroglia and microglia). Recent findings of variability in the expressions across genetically diverse rodent strains/lines suggest that genetic factors contribute significantly to the variation between patients in the clinical expression of neuropathic or inflammatory orofacial pain. Recent findings will also be presented that show that the $V_{c}$ central sensitization and associated nociceptive behaviour can also be attenuated by recently introduced drugs to manage orofacial pain states. Supported by NIH, CIHR, Pfizer Canada.

\section{B}

OROFACIAL AND TMD PAINS: SLEEP BREATHING, SLEEP BRUXISM AND PAIN INTERACTIONS IN OROFACIAL PAIN PATIENTS

Gilles J Lavigne

Faculté de médecine dentaire, Université de Montréal; Canada Research Chair Pain-Sleep-Trauma, Surgery Department, Hopital du Sacre Cour, Montreal, Quebec

Eight percent of the adult population report chronic orofacial pain and temporomandibular disorders (TMD), of which $25 \%$ to $50 \%$ also complain about poor sleep quality with few cases with insomnia, sleep breathing disturbances such as RERA (respiratory events related arousals) and sleep apnea syndrome. Morning Headache is also reported by general population in association with sleep bruxism (tooth grinding) or sleep apnea; causes are unknown (eg, poor breathing with low oxygenation-hypoxia, rise in $\mathrm{CO}_{2}$-hypercapnia).

In $25 \%$ to $50 \%$ of TMD cases, a circular relationship is suggested between a poor night's sleep and more pain the following day, and between too much pain during the day and a poor night's sleep. In fibromyalgia patients it is over $75 \%$ of cases that are presenting such a deleterious vicious circle. This simple relationship may be due to mood alterations, stress-related changes in the HPA axis, genetic predisposition, etc. To reduce overactivation during sleep, comorbidities must be excluded (sleep bruxism and sleep apnea) in sleep medicine. Patients should be instructed in good sleep hygiene strategies. It would also be useful for clinicians to work jointly with psychologists to correct certain beliefs and misunderstandings about pain and sleep. Medications can help normalize the propensity to stronger sleep arousal. Finally, oral appliances can reduce orofacial complaints and breathing problems in a sub-group of patients.

\section{$32 \mathrm{C}$}

\section{THE PHENOTYPIC AND GENETIC SIGNATURES OF TMD - LESSONS FROM THE OPPERA STUDY}

\section{Luda Diatchenko}

Department of Anesthesia, Faculty of Medicine, Faculty of

Dentistry, McGill University, Montreal, Quebec

Temporomandibular Disorders (TMD) tend to coexist with other chronic pain conditions in affected individuals and are characterized by a report of pain greater than expected based on the results of a standard physical evaluation. The pathophysiology of TMD is largely unknown, the scientific field lacks biological markers for accurate diagnosis, and conventional therapeutics have limited effectiveness. Growing evidence suggests that chronic pain conditions are associated with both physical and psychological triggers, which initiate pain amplification and psychological distress; thus, susceptibility is dictated by complex interactions between genetic and environmental factors. This presentation will review phenotypic and genetic markers of TMD as a result of a large study named OPPERA Orofacial Pain Prospective Evaluation and Risk Assessment - Study. The phenotypic markers of greatest interest include measures of pain amplification and 'psychological' measures (such as emotional distress, somatic awareness, psychosocial stress and catastrophizing). Genetic markers hve just started to be developed, however, polymorphisms reproducibly linked with musculoskeletal pain are found in genes contributing to serotonergic and adrenergic pathways. Elucidation of the biological mechanisms by which these markers contribute to the perception of pain in these patients will enable the development of novel effective drugs and methodologies that permit better diagnoses and approaches to personalized medicine.

\section{Session 303: 10:45 am - 12:15 pm}

\section{3}

COLLABORATION TEAM INITIATIVE BETWEEN TERTIARY AND PRIMARY CARE TO IMPROVE ACCESS AND DEVELOP ADAPTABLE MODELS OF PAIN MANAGEMENT PROGRAMS Chair: Lori Montgomery

Medical Director, AHS (Calgary) Chronic Pain Centre, Departments of Family Medicine and Anaesthesia, University of Calgary, Calgary, Alberta

Speakers: June S Bergman, Yolanda Martens Van Hilst WORKSHOP OBJECTIVE:

The population of chronic pain patients in the community is too large to be effectively managed in tertiary care alone. In addition, there is excellent evidence that patients prefer to see their primary care provider, and that behavioural change is most effectively achieved in this context. A team from Calgary will describe their models of care, challenges in implementation, and outcome data, in order to prompt discussion among our colleagues of best practices with regard to collaboration between tertiary and primary care pain clinicians. The team will facilitate a discussion in order to provide participants with hands on tools to apply principles and care models to their own environments.

Learning Objectives:

1. Be able to describe different models of primary care multi-disciplinary pain management teams and have the tools to apply it to their own setting.

2. Be able to describe potential challenges of these models and follow a method of problem solving to predict possible solutions.

3. Understand the importance of building partnerships between primary and tertiary care. 


\section{A}

\section{SHARED CARE MODELS IN PRIMARY CARE}

June Bergman

Associate Professor Emeritus, Department of Family Medicine, Faculty of Medicine, University of Calgary; Calgary Foothills Primary Care Network, Calgary, Alberta

Dr June Bergman is a family physician with a long history of program development and primary care development. She will speak to the underlying principles of local program design combined with tertiary expertise. She will explain the biopsychosocial model and integration of holistic and pain management approach in primary care and how this model has broadened the ability to manage a wide range of illness in the medical home. She will address partnerships and integrations between tertiary expertise and local program delivery; enhanced support for patients at the primary care level; and mechanisms of team integration as the patient moves from tertiary care to peripheral care to primary based care.

A particular emphasis will be on our experience of shared care models of chronic pain, mental health care, rheumatology, senior care and gastroenterology all employing similar models of tertiary expertise and local support for physicians and their teams as they care for complex patients.

\section{B}

\section{PAIN CAN BE WELL MANAGED IN PRIMARY CARE}

\section{Yolanda Martens Van Hilst}

\section{Calgary Foothills Primary Care Network, Calgary, Alberta}

Yolanda Martens-Van Hilst is the extended team manager for the Calgary Foothills Primary Care Network (CFPCN) and has been leading the collaborative pain team initiative in primary care since 2009. This pain initiative has led to a $20 \%$ decrease of referrals to tertiary care and has improved pain management in primary care. She will share her experience in exploring new and innovative ways of designing and delivering health care services in primary care. She will focus on building community partnerships and collaborating with specialist in tertiary care to ensure best practices. She will demonstrate the evolution from a multi-disciplinary team towards an interdisciplinary team and adapting varies models into the PCN medical home and communities. She will share the strategy of change, the implementation of the CFPCN pain initiative, challenges, lesson learnt and how the interprofessional, integrated chronic pain service delivery model impacts patients, providers, medical home and systems-level outcomes.

\section{Session 304: 10:45 am - 12:15 pm}

\section{4}

\section{NEUROTROPHINS AND PAIN}

Chair: Alfredo Ribeiro-da-Silva

Department of Pharmacology \& Therapeutics and Alan Edwards Centre for Research on Pain, McGill University, Montreal, Quebec Speakers: A Claudio Cuello, Alfredo Ribeiro-da-Silva,

Yves De Koninck WORKSHOP OBJECTIVE:

Understanding basic mechanisms that underlie the role of nerve growth factor (NGF) and brain-derived neurotrophic factor (BDNF) in arthritis and neuropathic pain.

Learning Objectives:

1. Understand how the knowledge of how NGF and BDNF are secreted from cells and metabolized can offer new therapeutic opportunities for the alleviation of pain

2. Understand the role of NGF in arthritis pain and how antagonizing it with antibodies or by reducing its endogenous formation from the precursor proNGF can alleviate pain

3. Understand the cascade of signalling events at the level of the spinal cord that can explain tactile allodynia

4. Identify components of signalling events that can be targeted for analgesia

5. Understand the role of the immune system in regulating excitability at the spinal level

\section{$34 \mathrm{~A}$}

\section{A NOVEL NGF METABOLIC PATHWAY AND ITS SIGNIFICANCE IN NEURODEGERATIVE DISORDERS AND PAIN}

\section{A Claudio Cuello}

Department of Pharmacology \& Therapeutics, McGill University, Montreal, Quebec

It was thought that the conversion of ProNGF to mature NGF (mNGF) occurred intracellularly and that this molecular form was released in an activity-dependent manner. Prior studies were performed in transfected cell lines or dissociated hippocampal cells utilizing ELISA techniques, an approach which does not differentiate proNGF from mNGF. Our lab instead resorted to the ex-vivo stimulation of superfused cortical tissue blocks. To our surprise, applying Western blotting, we observed that the material released in the extracellular space in an activity-dependent manner was consistently, ProNGF and not, as previously assumed, mNGF. Our observations supported the existence of a complex CNS metabolic pathway responsible for NGF conversion and degradation in the extracellular milieu (Bruno et al, PNAS, 2006). We have further validated this pathway in vivo by demonstrating that the inhibition of the Pro-to mature NGF conversion results in the brain accumulation of ProNGF concomitantly with a loss and atrophy of cortical cholinergic synapses (Allard et al, J. Neurosci. 2012). Furthermore, we have gathered neurochemical evidence for a compromise of this newly discovered NGF metabolic pathway in Alzheimer's disease (Bruno et al . J. Neuropathol. and Exp. Neurol. 2009) and in Down Syndrome (Iulita et al Brain, 2014), thus explaining the vulnerability of NGF-dependent forebrain cholinergic neurons in these neurodegenerative conditions, despite normal NGF synthesis and abundance of NGF precursor. Furthermore, we have proposed that this pathway might also be altered in peripheral inflammatory pain and that the knowledge of this novel metabolic pathway could offer new therapeutic opportunities.

\section{B}

\section{NGF AND ARTHRITIS PAIN}

Alfredo Ribeiro-da-Silva

Department of Pharmacology \& Therapeutics and Alan Edwards Centre for Research on Pain, McGill University, Montreal, Quebec

The role of NGF in arthritis pain has been well documented, and NGFsequestering antibodies were used in clinical trials to control pain in osteoarthritis patients. We have been investigating an alternative approach based on the discovery that NGF is released in its precursor form (proNGF) and then processed extracellularly into mature $\mathrm{NGF}(\mathrm{mNGF})$ by plasmin. We have been using a rat inflammatory monoarthritis model in which complete Freund's adjuvant (CFA) is injected into the ankle joint. At four weeks post-CFA, in the skin adjacent to the arthritic joint, there was an increase of mNGF levels and sprouting of sympathetic fibres. In the synovial membrane, both nociceptive peptidergic and sympathetic fibres sprouted. To assess whether sympathetic fibres played a role in the pain, we injected guanethidine at two and four weeks post-CFA. Guanethidine led to a partial alleviation of the pain-related behaviour at four weeks, but not at two weeks (a time-point in which there is no sympathetic sprouting). As a proof of principle, we injected an inhibitor of mNGF degradation into the hindpaw skin of naïve rats. This treatment induced sympathetic sprouting and hyperalgesia, similar to what is seen in our arthritis model. Finally, using $\alpha 2$-antiplasmin, we blocked the maturation of proNGF into mNGF in animals with monoarthritis of the ankle joint, and saw a reduction in mNGF levels and an alleviation of the hyperalgesia. Our observations indicate that the reduction of abnormally elevated levels of mNGF may represent a valid novel therapeutic approach for arthritis.

\section{$34 \mathrm{C}$}

\section{BDNF, CHLORIDE AND PAIN}

Yves De Koninck

Institut universitaire en santé mentale de Québec, Université Laval, Quebec

Weakened GABAA and glycine receptor-mediated inhibition is an important mechanisms underlying enhanced excitability in the dorsal horn of 
the spinal cord following peripheral nerve injury. An emerging mechanism by which the strength of inhibition is adjusted is via alterations in $\mathrm{Cl}-$ homeostasis. The resulting weakened inhibition enables a cross-talk between sensory pathways allowing the aberrant relay of innocuous input through normally nociceptive specific pathways, setting the stage for pain hypersensitivity. The identification of the signalling events and ionic mechanisms involved in neuron-glia-neuron interactions after nerve injury offers novel avenues for treatment of neuropathic pain.

It now emerges that the transmembrane gradient for $\mathrm{Cl}-$ ions in adult dorsal horn neurons is actively regulated by endogenous neuromodulators, such as BDNF. The source of BDNF at the spinal level, after peripheral nerve injury, appears to be microglia. Indeed, nerve injury causes the release of the chemokine MCP-1 (or CCl2) from sensory terminals in the spinal dorsal horn, which triggers activation and chemotaxis of microglia via activation of the CCR2 receptor. Activated microglia begin to express P2X4 receptors. Spinal ATP release then causes microglia to secrete BDNF, which acts on $\operatorname{TrkB}$ receptors located on superficial dorsal horn neurons to cause a downregulation of the $\mathrm{K}+-\mathrm{Cl}-\mathrm{co}$-transporter $\mathrm{KCC} 2$, weakening $\mathrm{Cl}-$ extrusion in cells with the end effect of impairing inhibition. Importantly, blocking BDNF-TrkB signalling reverses the deficit in $\mathrm{Cl}-$ transport and tactile allodynia. Thus, sustained secretion of BDNF is necessary to maintain weakened $\mathrm{Cl}-$ extrusion in neurons indicating that targeting this signalling pathway may be an efficient strategy to reverse established pathological pain.

\section{Session 305: 1:00 pm - 2:30 pm}

\section{5}

\section{PAIN MEMORY - THE CONTRIBUTION OF EARLY LIFE PAINFUL EXPERIENCE TO ADULT PAIN SENSITIVITY}

Chair: Simon Beggs

Programmes in Brain and Behaviour, The Hospital for Sick Children; Faculty of Dentistry, University of Toronto, Toronto, Ontario

Speakers: Simon Beggs, Mark Baccei, Mike Costigan WORKSHOP OBJECTIVE:

Tissue injury in early postnatal life has long-term consequences on subsequent pain processing that can persist into adult life. Using genetic, electrophysiological and behavioural techniques this symposium will explore the mechanisms and interactions that underlie this at the molecular, cellular and systems level.

Learning Objectives:

1. To understand the importance of the contribution of the immune system to nociceptive processing in young and adult animals.

2. Gain insight into the long-term consequences of neonatal tissue damage for synaptic signaling in nociceptive circuits of the adult CNS.

3. Gain an understanding of the molecular mechanisms that control the processes that lead to neuropathic pain.

\section{A}

\section{NEUROIMMUNE INTERACTIONS UNDERLYING THE LONG-TERM CONSEQUENCES OF EARLY LIFE INJURY}

\section{Simon Beggs}

Programmes in Brain and Behaviour, The Hospital for Sick Children; Faculty of Dentistry, University of Toronto, Toronto, Ontario

Peripheral nerve injury in the early postnatal period has profound effects on pain sensitivity to subsequent injury in later life. A complex interaction between the developing immune and nervous systems contributes to these long-term effects. In this symposium the effects of peripheral nerve damage on immune cells within the CNS will be explored and how these cells mediate the behavioural response to subsequent injury.

\section{B}

LONG-TERM CONSEQUENCES OF NEONATAL TISSUE DAMAGE ON NOCICEPTIVE SYNAPTIC SIGNALING IN THE ADULT CNS

Mark Baccei

Department of Anesthesiology, University of Cincinnati, Cincinnati, Ohio, USA

Tissue injury during a critical period of early postnatal development can alter pain sensitivity throughout life. However, the degree to which neonatal tissue damage exerts prolonged effects on synaptic transmission within adult spinal nociceptive circuits remains unclear. This seminar will review recent evidence suggesting that transient tissue injury during the neonatal period can evoke long-term deficits in inhibitory signaling within the adult mouse superficial dorsal horn $(\mathrm{SDH})$, which represents a key relay station along the pain pathway. We will also examine the persistent effects of early tissue damage on the strength and plasticity of primary afferent inputs to adult lamina I projection neurons, which serve as an important output of the spinal pain network and are known to be essential for the generation of inflammatory and neuropathic hypersensitivity. Overall, the data suggest that aberrant sensory input during early life has permanent consequences for the functional organization of nociceptive synaptic circuits within the adult spinal cord.

\section{$35 \mathrm{C}$}

\section{WHOLE GENOME EXPRESSION ANALYSIS TO IDENTIFY THE MOLECULAR MEDIATORS OF NEUROPATHIC PAIN Mike Costigan \\ Department of Anaesthesia, Boston Children's Hospital, Boston, Massachusetts, USA}

In this symposium the molecular response of peripheral neurons to injury will be explored. Using expression microarrays to identify regulated genes in damaged sensory neurons en masse, we aim to define the changes in the molecular mechanisms that underlie neuropathic pain in young and old animals which, as in humans, develop chronic pain post nerve injury when mature but to a much lesser degree in neonates. These changes can be broadly split into adaptive alterations including neuronal survival and regeneration and maladaptive changes which include chronic pain. The aim of the research outlined in this symposium is to find and characterize the molecules that control these processes. Understanding the negative consequences of nerve injury, particularly neuropathic pain is a major research goal towards developing novel pain therapeutics. Current genetic profiling work includes performing genome-wide single nucleotide polymorphism (SNP) association studies in patient cohorts, these extremely exciting studies offer a window for the first time on the actual molecules important to the development of neuropathic pain. In ongoing work we are confirming our primary genome wide association (GWAS) data in novel patient cohorts. These studies promise, once done, to be the first encyclopedia of human chronic pain genes.

\section{Session 306: 1:00 pm - 2:30 pm}

\section{6}

\section{A DEBATE AROUND THE ETHICS OF ONLINE TREATMENTS FOR PAIN}

Chair: Patrick McGrath

IWK Health Centre, Capital District Health Authority, Dalhousie

University, Halifax, Nova Scotia

Speakers: Ellen M Henderson, Brian McGuire

WORKSHOP OBJECTIVE:

To debate the ethics of online treatments for pain with respect to the role of the therapist in research and in standard care

Learning Objectives:

1. To present the debate for greater added regulations and accountability criterion for therapy delivered online in one 20 minute presentation.

2. To present the debate for no added regulation for therapy presented online in one 20 minute presentation. 
3. To come to consensus regarding the regulation of pain therapy presented online.

\section{A}

\section{THE CASE FOR REGULATION}

\section{Ellen $M$ Henderson}

Louis Dundas Centre for Children's Palliative Care, University College London, London, United Kingdom

Offline ethical guidance for the presentation of therapy has clear boundaries in place for the delivery of care. These guidelines oblige the therapist with a responsibility to the patient should they present as if to harm themselves or others, to provide a good standard of care to the patient within a specific time frame and to be licensed to practice as their patient's therapist in a specific geographical location. When therapy is presented through the Internet the therapist can through the very organization of the delivery of care not always decipher if their patient will harm themselves or others and may not be able to act as quickly in the geography in which the patient is placed. Indeed the therapist may not even be licensed to practice in the geographical locations where the therapy is taking place - in the patient's own home, perhaps in another state or country. The implications for both patient and therapist should something go wrong are unexplored. Increased regulation should be set out to protect both sides in the patient client relationship and be clear on what the governance expects of this relationship before the field should be allowed to advance.

\section{B}

\section{THE CASE AGAINST}

\section{Brian McGuire}

School of Psychology and Centre for Pain Research, The National University of Ireland, Galway, Ireland

It is argued that current ethical guidelines are adaptable to diverse contexts including the use of online treatments for pain. Ethical standards developed in advance of the internet have undergone several revisions in the years since researchers began engaging in research methodologies utilizing information and communication technology. While the ethical research guidance in place does not directly refer to the ethical issues of therapy delivered online, prominent ethical concerns in internet-based research can be understood in terms of established ethical research concepts. The application of these concepts to prominent issues in internet research will be discussed in relation to several multi-phase, mixed-methods studies carried out at our centre, including both qualitative studies which involve assessing consumer perspectives about online therapy and quantitative studies aimed at evaluating the feasibility, acceptability and effectiveness of online pain management programs. An argument is made that, with due regard to current ethical guidelines, there is a strong imperative to make effective treatments available to as many people as possible.

\section{Session 307: 1:00 pm - 2:30 pm}

\section{7}

\section{OPIOIDS FOR PAIN 2014 - AN UPDATE}

\section{Chair: Roman D Jovey}

CPM Centres for Pain Management, Mississauga, Ontario

Speakers: Roman D Jovey, Allan S Gordon

WORKSHOP OBJECTIVE:

The attendees will critically appraise and put into perspective the recent publicity regarding opioid related harms and enhance their strategies for safely prescribing opioids to patients who may benefit from opioids for chronic pain management.

Learning Objectives:

1. Critically appraise the recent adverse publicity regarding opioid-related harms.

2. Develop a clinical strategy to optimize outcome and reduce the harms of opioid therapy.

3. Maintain a balanced view of the pros and cons of prescribing opioids for CNCP.

\section{A}

OPIOIDS - ASSESSING HARMS, RESTORING BALANCE

Roman D Jovey

CPM Centres for Pain Management, Mississauga, Ontario

There has been much recent adverse publicity focused on the risks of opioids and the adverse effects related to therapeutic use as well as the harms related to diversion, misuse and addiction. The net result has been the reluctance of a growing number of primary care practitioners to take on patients with chronic pain and the outright refusal to prescribe long-term opioid therapy - even in modest amounts. This presentation will critically examine some of the publicized harms of opioids for pain management in an attempt to restore balance to the discussion.

\section{B}

\section{THE SEVEN STAGES OF OPIOID PRESCRIBING (REVISITED)}

\section{Allan S Gordon}

Wasser Pain Management Centre, Mount Sinai Hospital, University of Toronto, Centre for the Study of Pain, Toronto, Ontario

In the late 90's and early 2000's, it was thought that opioid prescribing for chronic non-cancer pain was fairly simple. That belief did not last long and the concept of the Seven Stages was originated in 2005 and first presented in 2007, as a description of opioid prescribing habits in the early part of the 2000-2010 decade. The Seven Stages included: 1, Opioid Naïve; 2, Opiophobic; 3, Opiophilic; 4, Opioid Expert; 5, Opioid Disaster; 6, Acquired Opiophobia; and 7, Opioid Balance. With appropriate precautions, opioids can still be very effective medications for treating chronic pain. This presentation will utilize case presentations to illustrate an updated version of the Seven Stages. The goal is to optimize the benefits and reduce the risks of opioid therapy for chronic pain.

\section{Session 308: 1:00 pm - 2:30 pm}

\section{8}

\section{BIOPSYCHOSOCIAL INFLUENCES ON CHRONIC PAIN FOLLOWING WHIPLASH INJURY: WHAT DO EVIDENCE AND EXPERIENCE TEACH US ABOUT MODULATING THE SYMPTOMS OF CHRONIC WHIPLASH?}

Chair: Ashley Smith

Division of Physiotherapy, NHMRC Centre of Clinical Excellence in Spinal Pain, Injury and Health, University of Queensland, Brisbane, Australia

Speakers: Ashley Smith, Whitney Scott, Pamela M Barton WORKSHOP OBJECTIVE:

Whiplash is a heterogeneous condition with subgroups of patients presenting with a poor prognosis. Prospective studies indicate that approximately $20 \%$ to $30 \%$ of patients continue to experience significant pain two to three years following the initial injury. Accumulating evidence suggests that chronic pain and disability associated with whiplash injury may be best understood from a biopsychosocial perspective. This symposium will provide attendees with clinical research findings pertaining to the physical and psychosocial correlates of chronic pain following whiplash injury. Knowledge from recent scientific evidence translated to clinical experience will be provided to help clinicians effectively manage patients with persistent pain and disability following whiplash injury.

Learning Objectives:

1. To provide an overview of the physical manifestations of individuals with persistent pain following whiplash injury, and identify current treatment options that have been demonstrated to improve outcomes in these patients.

2. To identify psychosocial factors that have been shown to influence the persistence of pain following whiplash injury. Discuss research on interventions targeting these psychosocial factors to improve pain-related outcomes in these patients.

3. To describe pragmatic pharmacological strategies for the management of individuals with persistent pain following whiplash injury with a focus on identifying and treating those persons with central sensitivity. 
38A

CAN THE PHYSICAL MANIFESTATIONS OF WAD BE MODULATED?

Ashley Smith

Division of Physiotherapy, NHMRC Centre of Clinical Excellence in Spinal Pain, Injury and Health, University of Queensland, Brisbane, Australia

Various treatments for individuals with persistent WAD have only shown modest effectiveness. Literature identifying the cervical facet joint as a source of nociception in individuals with persistent WAD highlights the importance of 'biological' in the biopsychosocial model of WAD management. Emerging evidence suggests that reducing peripheral nociception, (cervical facet joint pain), results in improvements in pain and disability, sensory hypersensitivity and psychological manifestations. As such, the early assessment and identification of peripheral pain mechanisms, such as the cervical facet joint is necessary for both targeted intervention strategies and/or appropriate referral.

Implications: Recognizing specific impairments that can be modulated is essential to reduce pain and disability in individuals with persistent WAD. Awareness of validated treatment options by healthcare providers assists in reduction of costs associated with managing WAD.

\section{B}

PSYCHOSOCIAL PREDICTORS OF PERSISTENT PAIN AND DISABILITY FOLLOWING WHIPLASH INJURY: RESEARCH AND CLINICAL IMPLICATIONS

Whitney Scott McGill University, Montreal, Quebec

Research indicates that psychosocial variables have an important influence on the persistence of pain and disability following whiplash injury. This presentation will review research on the role of pain catastrophizing and perceived injustice in recovery following whiplash injury. Research examining the impact of mental health symptoms will also be reviewed. Psychosocial interventions that may be useful for targeting these risk factors will be described. Data will be presented supporting the usefulness of psychosocial interventions for improving pain-related outcomes in patients with whiplash injuries. Implications: Early screening for psychosocial risk factors and subsequent implementation of risk factor targeted interventions may contribute to improved pain-related outcomes following whiplash injury.

\section{$38 \mathrm{C}$}

MEDICATION STRATEGIES FOR INDIVIDUALS WITH PERSISTENT PAIN FOLLOWING WHIPLASH INJURY

\section{Pamela Barton}

Alberta Health Services Chronic Pain Centre; Hotchkiss Brain Institute; Division of Physical Medicine and Rehabilitation, University of Calgary, Calgary, Alberta

Astute clinical history and physical examination techniques can help identify potential central sensitization and neuropathic pain components in individuals with chronic WAD. Once identified, there are rational and pragmatic pharmacologic strategies which may contribute to improvement in pain symptoms. The choice, role and sequencing of medication in facilitating other components of interdisciplinary management will be explored. Implications: Medications, appropriately chosen and monitored, can augment interdisciplinary care and reduce the overall pain experience. 
CPS POSTERS, 2014

WEDNESDAY MAY 21, 2014

\section{P1}

\section{WITHDRAWN}

\section{P2}

\section{BEHAVIORAL SIGNS OF PAIN IN A MOUSE MODEL OF OSTEOGENESIS IMPERFECTA}

Dareen M Abdelaziz ${ }^{1}$, Sami Abdullah'², Robert Samberg ${ }^{3}$, Svetlana V Komarova ${ }^{2,4}$, Frank Rauch ${ }^{2,5,6}$, Laura S Stone ${ }^{3,6,7,8}$ ${ }^{1}$ McGill University; ${ }^{2}$ Shriners Hospitals for Children ${ }^{\circledR}$-Canada; ${ }^{3}$ Alan Edwards Centre for Research on Pain; ${ }^{4}$ Faculty of Dentistry; ${ }^{5}$ Department of Pediatrics; ${ }^{6}$ Department of Anesthesiology; ${ }^{7}$ Department of Pharmacology \& Therapeutics, ${ }^{8}$ Department of Neurology \& Neurosurgery, Faculty of Medicine, McGill University, Montreal, Quebec

AIM: Osteogenesis imperfecta (OI) is a congenital disorder primarily affecting bone. Pain is common in OI patients which impedes their lives. Managing OI pain remains suboptimal even with available approaches. Our aim is to better understand the sources of pain in this syndrome and to question the effectiveness of possible treatments.

METHODS: The recently developed OI model (Colla1Jrt/+) was assessed for weight, skeletal events and behavioral signs of pain compared to their wild type (WT) littermates. Two- to three-month-old 14 Colla1Jrt/+ and 15 WT males were tested for mechanical, radiant heat and cold sensitivities. Motor activity and anxiety-like behavior were evaluated using home cage running wheels for $1 \mathrm{~h}$ and open field for $5 \mathrm{~min}$, respectively. Additionally, the effect of bisphosphonate (a current treatment for OI patients) on sensory thresholds and motor abilities was examined. Three injections of Zometa $(100 \mu \mathrm{g} / \mathrm{kg})$ or saline were given i.p. to Colla1Jrt/+ ( $\mathrm{n}=7$ per group) and WT ( $n=5$ to 10 per group) over three weeks.

RESULTS: Our data demonstrated significant differences between the two groups in weight progression and skeletal abnormalities including undersized long bones, joint displacement, vertebral column misalignment and small caudal vertebrae. Statistically significant differences were observed between the groups in all tests of sensory hypersensitivity and motor ability. Preliminary results of bisphosphonate treatment reveal insignificant effects on mechanical or cold thresholds but strong reduction in heat hypersensitivity.

CONCLUSIONS: Increased sensitivity in Colla1Jrt/+ might be due to several factors including bone dislocations, joint hyperlaxity and nerve compression. Bisphosphonate treatment may be beneficial in reducing sensitivity associated with skeletal deformities but their analgesic efficacy requires further investigation.

\section{P3}

\section{INTRATHECAL MULTIDRUG INFUSION FOR PAIN CONTROL IN ELDERLY PATIENTS AND END-STAGE MALIGNANCIES - THREE CASE REPORT SERIES}

\section{Sadegh Abdolmohammadi, Gilbert Blaise}

Anesthesiology and Pain Medicine, CHUM, Montreal University, Montreal, Quebec

AIM: To present an intrathecal infusion method for pain relief in elderly and end stage malignancies

METHODS: Three elderly patients (64 to 94 years of age), admitted in hospital for intractable pain due to metastatic malignancy or fracture -A preservative-free mixture of bupivacaine $1 \mathrm{mg} / \mathrm{mL}$, naloxone $0.02 \mathrm{ng} / \mathrm{mL}$, ketamine $100 \mu \mathrm{g} / \mathrm{mL}$, morphine $0.01 \mathrm{mg} / \mathrm{mL}$ and clonidine $0.75 \mu \mathrm{g} / \mathrm{mL}$ was infused by an external pomp with a rate of $1 \mathrm{~mL} / \mathrm{h}$ to $2 \mathrm{~mL} / \mathrm{h}$ via an intrathecal catheter

-Decreasing rate during the following days due to patients' requirement. -Mixture stability was assessed during five days

RESULTS: -In all patients, pain was successfully controlled without any major complication (lower limb muscles weakness, sphincter dysfunction, constipation and cognitive or mood dysfunction)
-In two patients, catheter was removed after four weeks before leaving hospital

-The third one with cancer still benefices from the treatment after six months at home

CONCLUSIONS: Naloxone in ultra low-dose helps controlling pain and prevents from hyperalgesia $(1,2)$. Ketamine could be used as intrathecal in end-stage cancer related pain. Our doses are much less than what have been already recommended (3). Absorption and systemic effect could not explain analgesic effect of the mixture. Synergic effect and different mechanisms of action could explain sufficient analgesia. The other advantage of our mixture is lower risk of neurotoxicity. We succeeded to control severe pain with a very low daily intrathecal dose without major complication

Footnotes/References:

1. J Opioid Manag 2008;4:251-4.

2. Expert Opin Investig Drugs 2013;22:517.

3. Med Clin North Am 2007;91:251-70.

\section{P4}

NON DERMATOMAL SENSORY ABNORMALITIES ASSOCIATED WITH PARALYSIS AND INTRACTABLE PAIN: TWO EXTREME CASE REPORTS

Maha Alkokani, Nivan Zoheiry, Angela Mailis Gagnon

Comprehensive Pain Program, Toronto Western Hospital/University Health Network, Toronto, Ontario

AIM: To understand complex non-dermatomal sensory abnormalities in the presence of pain and emotional distress

METHODS: We present two extreme cases seen in a tertiary care pain clinic RESULTS: Case presentation: 1) A 28-year-old woman suffered immediate left leg pain and loss of sensation/power in a frightening car accident which caused soft tissue injuries. $\mathrm{O} / \mathrm{E}$ three years later, the left foot was anesthetic with permanent inversion contractures, complete lack of voluntary movements, and colder by $4^{\circ} \mathrm{C}$ compared to the right. Under IV sodium pentobarbital she had minimal improvement in sensation but no return of movement. 2) A 43-year-old woman suffered a left wrist fracture in a car/pedestrian accident. She experienced intense feelings the arm was not hers and refused to look at it. O/E 1.5 year later the left arm was motionless and powerless, with intense hyperpathia in the left face, neck and arm. IV sodium pentobarbital resulted in dramatic but temporary resolution of allodynia and less so hyperalgesia, but no return of movement. In both cases investigations failed to disclose structural peripheral or central neurological lesion. Both patients were diagnosed with severe post traumatic stress disorder (PTSD,) major depression (MD) and conversion disorder (CD).

CONCLUSIONS: While psychotraumatic experiences associated with variable degrees of physical trauma, anesthesia, and paralysis are the hallmark of CD, our cases demonstrate that both hyperesthesiae and hypoesthesiae of the paralyzed limb can be associated with $\mathrm{CD}$ in conjunction with PTSD and MD. Functional imaging data on exiting literature will be discussed.

\section{P5}

\section{THE PREDICTIVE ROLE OF PAIN IN PTSD SYMPTOMS}

Nelly Amariglio, Rika ML Meyer, Jeffery I Gold

Children's Hospital Los Angeles, Los Angeles, California, USA

AIM: This study investigated the role of pain in predicting the development and maintenance of PTSD symptoms among adult patients with cancer.

METHODS: A total of 285 adult patients with cancer who were recruited from VA medical centers completed the PTSD Checklist - Civilian Version (PCL-C), the Brief Pain Inventory, and the bodily pain concept from the Functional Health Status measure (SF-36).

RESULTS: All six domains of pain predicted significantly greater PTSD symptoms ( $\mathrm{b}$ range $=0.070$ to 1.83 , P range $=0.002$ to 0.06 ). Additionally, differences between patients who met criteria for full PTSD versus no PTSD were found in five of the six pain domains: pain interferes with work $(\mathrm{P}=0.002)$, worst pain in the past seven days $(\mathrm{P}=0.002)$, least pain in the 
past seven days $(\mathrm{P}=0.02)$, average pain in the past seven days $(\mathrm{P}=0.002)$, and pain right now $(\mathrm{P}=0.02)$. All three DSM-IV PTSD clusters (reexperiencing, avoidance/numbing, and hyper-arousal) were significantly related to five of the six pain domains. Similarly, according to DSM-V criteria for PTSD, there was a positive correlation between pain and three out of four PTSD clusters (intrusion, negative mood, and arousal). No correlation was found between the avoidance cluster and pain.

CONCLUSIONS: Pain appears to be a strong predictor of PTSD symptoms among adult patients with cancer who are undergoing medical treatment. Pain measures may be utilized as an effective screener for PTSD among medically fragile populations.

\section{P6}

\section{ONLINE MINDFULNESS-BASED STRESS REDUCTION FOR THE MANAGEMENT OF CHRONIC HEADACHE PAIN IN ADULTS}

Brian McGuire ${ }^{1,2}$, Jonathan Egan ${ }^{1}$, Angeline Traynor ${ }^{1}$

${ }^{1}$ School of Psychology; ${ }^{2}$ Centre for Pain Research, National University of Ireland, Galway, Ireland

AIM: To evaluate the efficacy of an online mindfulness based stress management program tailored for adults with chronic headache.

METHODS: A total of 221 participants (18 to 65 years of age) took part in a one group, pre-test to post-test internet based intervention for chronic headache management. An internet based program offering tailored mindfulness-based techniques and practical advice for self-management of chronic headache was developed (http://www.headachemanagement.org). A different theme and mindfulness exercise was introduced in each week of the six week program including: information about chronic headache, progressive muscle relaxation, guided visualization, sleep and headache onset relaxation. The primary treatment outcomes were pain severity and pain interference (Brief Pain Inventory), anxiety and depression (Hospital Anxiety and Depression Scale), headache impact (Headache Impact Test6), medication intake and programme uptake. All participants selfreported their chronic headache diagnosis, they were predominantly tension-type headache and chronic migraine. Recruitment, data collection, data analyses were all performed online.

RESULTS: Of those completing the program $(n=71), 78 \%$ were female. Findings demonstrated clinically significant pre-to-post reductions in pain severity $(r=0.69)$, pain interference $(r=0.59)$, anxiety $(r=0.66)$, depression $(\mathrm{r}=0.51)$, headache impact $(\mathrm{r}=0.53)$ and medication intake $(\mathrm{r}=0.54)$. Preto-post reduction in healthcare service treatment-seeking behaviour was not statistically significant. Attrition was considerably higher than expected at $70 \%$.

CONCLUSIONS: If the problem of participant attrition can be addressed, the results suggest that a relatively short online mindfulness-based selfmanagement program may be clinically effective for people with chronic headache, with benefits observed in pain severity and interference, anxiety and depression, and medication usage. Further research to assess these effects in a larger sample is underway. Indications of efficacy must be considered with caution, because they are based on an uncontrolled pre-post design.

\section{P7}

CAN FLUCTUATIONS IN VITAL SIGNS BE USED FOR PAIN ASSESSMENT IN CRITICALLY ILL PATIENTS WITH A TRAUMATIC BRAIN INJURY?

Caroline Arbour $^{1}$, Manon Choinière ${ }^{2,3}$, Jane Topolovec-Vranic ${ }^{4}$, Mélody Ross ${ }^{1}$, Carmen G Loiselle ${ }^{1,5}$, Céline Gélinas ${ }^{1,5}$

${ }^{1}$ Ingram School of Nursing, McGill University; ${ }^{2}$ Centre de recherche de Centre hospitalier de I'Université de Montréal (CRCHUM);

${ }^{3}$ Université de Montréal, Department of Anaesthesiology, Montreal, Quebec; ${ }^{4}$ Trauma and Neurosurgery Program and Keenan Research Center of the Li Ka Shing Knowledge Institute, St Michael's Hospital, Toronto, Ontario; ${ }^{5}$ Centre for Nursing Research and Lady Davis Institute - Jewish General Hospital, Montreal, Quebec

AIM: Many patients with a traumatic brain injury (TBI) are not able to communicate in the intensive care unit (ICU). While behaviors are recommended for pain assessment in nonverbal patients, they are unusable in TBI patients who are under neuroblocking agents. In this situation, vital signs are the only cues left for pain assessment. This study described vital signs fluctuations in TBI patients during common procedures in the ICU. METHODS: A repeated-measure, within-subject design was used. Participants ( $n=45$ ) were observed for 1 min before (baseline), during, and 15 min after two procedures: 1) non-nociceptive (non-invasive blood pressure-NIBP), and 2) nociceptive (turning). At each assessment, vital signs (ie, systolic, diastolic, mean arterial pressure (MAP), heart rate (HR), respiratory rate $(\mathrm{RR})$, capillary saturation $(\mathrm{SpO} 2)$, end-tidal $\mathrm{CO}_{2}$, and intracranial pressure (ICP) were recorded continuously using a data collection computer (Moberg-CNS monitor, PA, USA).

RESULTS: Overall, most vital signs had slightly higher mean values during turning than at baseline or during NIBP. Although significant fluctuations $(\mathrm{P}<0.05)$ in diastolic, $\mathrm{SpO}_{2}$, and ICP were found across assessments, they were similar during NIBP and turning and were not always correlated to participants' self-report. In contrast, RR was found to increase exclusively $(\mathrm{P}<0.05)$ during turning and was positively correlated to participants' self-report ( $\mathrm{rpb}=0.736)$.

CONCLUSIONS: Findings from this study support previous ones that vital signs could be of little utility for the detection of pain in critical care. While RR could be an indicator of pain in TBI patients, further research is warranted to examine its validity.

\section{P8}

\section{DETECTING PAIN IN TRAUMATIC BRAIN INJURED PATIENTS WITH DIFFERENT LEVEL OF CONSCIOUSNESS DURING COMMON PROCEDURES IN THE ICU: TYPICAL OR ATYPICAL BEHAVIORS?}

Caroline Arbour ${ }^{1}$, Manon Choinière ${ }^{2,3}$, Jane Topolovec-Vranic ${ }^{4}$, Mélody Ross ${ }^{1}$, Carmen G Loiselle ${ }^{1,5}$, Kathleen A Puntillo ${ }^{6}$, Céline Gélinas ${ }^{1,5}$

${ }^{1}$ Ingram School of Nursing, McGill University; ${ }^{2}$ Centre de recherche de Centre hospitalier de I'Université de Montréal (CRCHUM); ${ }^{3}$ Université de Montréal, Department of Anaesthesiology, Montreal, Quebec; ${ }^{4}$ Trauma and Neurosurgery Program and Keenan Research Center of the Li Ka Shing Knowledge Institute, St Michael's Hospital, Toronto, Ontario; ${ }^{5}$ Centre for Nursing Research and Lady Davis Institute, Jewish General Hospital, Montreal, Quebec; ${ }^{6}$ University of California San Francisco, School of Nursing, San Francisco, California, USA

AIM: Pain behaviors such as grimacing and frowning are recommended for pain assessment in nonverbal populations. However, these behaviors may not be appropriate for critically ill patients with a traumatic brain injury (TBI) depending on their level of consciousness (LOC). This study aimed to validate the use of pain behaviors for pain assessment in critically ill TBI adults with different LOC.

METHODS: Using a repeated measure within subject design, participants $(\mathrm{n}=45)$ were observed for 1 minute before (baseline), during, and $15 \mathrm{~min}$ after two procedures: 1) non-invasive blood pressure: NIBP (non-nociceptive), and 2) turning (nociceptive). A 50 -item behavioral checklist and video recording were used to document participants' behaviors. Intra and inter-rater agreement of observed behaviors was examined.

RESULTS: Pain behaviors were observed more frequently during turning ( median=4; $\mathrm{T}=-5.336 ; \mathrm{P}<0.001$ ) than at baseline (median=1), or during NIBP (median=0). Pain behaviors were mostly 'atypical' and included flushing, sudden eye opening, eye weeping, and flexion of lower limbs. These behaviors were noted in $\leq 25 \%$ of unconscious TBI participants and in up to $66.7 \%$ of conscious ones who reported the presence of pain. Interestingly, grimacing - a pain behavior included in clinical guidelines for pain assessment - was documented in less than $12.0 \%$ of participants. Agreements were $>92 \%$ among and between raters.

CONCLUSIONS: This study support emerging evidence that critically ill TBI patients exhibit atypical behaviors during nociceptive procedures. As such, use of current recommended pain behaviors and behavioral pain scales may not be optimal for assessing the analgesic needs of TBI patients independent of their LOC. 


\section{P9}

GREY MATTER DENSITY AND CHRONIC PAIN IN THE MONTHS FOLLOWING MODERATE TO SEVERE TRAUMATIC BRAIN INJURY: INSIGHTS FROM PRELIMINARY FINDINGS

Caroline Arbour ${ }^{1}$, Andrée-Ann Baril ${ }^{1,2}$, Pierre-Olivier Gaudreault ${ }^{1,3}$, Catherine Duclos $^{1,3}$, Gilles J Lavigne ${ }^{1,4}$, Nadia Gosselin ${ }^{1,3}$

${ }^{1}$ Center for Advanced Research in Sleep Medicine (CARSM), Hôpital du Sacré-Coeur de Montréal; ${ }^{2}$ Department of Psychiatry; ${ }^{3}$ Department of Psychology; ${ }^{4}$ Faculty of Dental Medicine; Université de Montréal, Montreal, Quebec

AIM: Chronic pain is common after a traumatic brain injury (TBI). While the role of the brain in chronic pain remains poorly understood, reduction of grey matter density was observed in the thalamus and prefrontal cortex in non-brain injured adults with persistent pain. Here, we present preliminary findings from an ongoing study aimed to explore the associations between grey matter density and chronic pain among TBI survivors.

METHODS: Magnetic resonance imaging (MRI) data were acquired with a $3 \mathrm{~T}$ scanner. Grey matter density was estimated using voxel-based morphometry (VBM). Age-corrected correlations between regional grey matter density and average pain intensity experienced in the last $24 \mathrm{~h}$ (reported on a 0 to 10 visual analogue scale) were assessed using VBM8 toolbox for statistical parametrical mapping software (SPM8).

RESULTS: Nine adults (eight males, one female) with a mean age of $34.33 \pm 14.44$ years were recruited 10 to 36 months after moderate to severe TBI. Eight participants reported the presence of pain in the last $24 \mathrm{~h}$ at a mean intensity of $3.25 \pm 1.25$. A significant negative correlation $(r=-0.96$; $\mathrm{P}=0.012$ ) was found between pain intensity and grey matter density in the right uncus (Brodmann area: 28). A trend for a negative correlation $(\mathrm{r}=-0.93 ; \mathrm{P}=0.06)$ was also found between pain intensity and grey matter density in the left uncus (Brodmann areas: 28, 36).

CONCLUSIONS: Our preliminary results suggest that chronic pain after moderate/severe TBI is accompanied by bilateral reduction of gray matter density in the uncus - a region of the limbic lobe. Thus, the pathophysiology of chronic pain after TBI could involve dysfunctions in the affective self-regulatory mechanisms.

\section{P10}

\section{INCIDENCE OF INTRAVENOUS PATIENT CONTROLLED ANALGESIA AND CONTROLLED RELEASE OPIOIDS INITIATION FOLLOWING TOTAL KNEE ARTHROPLASTY}

Arlene Buzon-Tan, Susan Walker, Kyle Kirkham

Acute Pain Service, Department of Anesthesia \& Pain Management, Toronto Western Hospital, University of Toronto, Toronto, Ontario AIM: The objective of this quality assurance review is to describe the postoperative pain management practices of the Acute Pain Service at our tertiary care academic hospital (Toronto Western Hospital) in the total knee arthroplasty (TKA) population. In particular, we sought to determine the percentage of patients who required initiation of intravenous patient controlled analgesia (IV PCA) and controlled release (CR) opiates.

METHODS: A prospective quality assurance audit of 500 consecutive patients undergoing TKA was conducted. Current clinical practice includes spinal anesthesia with intrathecal morphine, intra-articular local anesthetic infiltration, oral opioids as needed, acetaminophen and NSAIDS. We examined the frequency of initiation of rescue IV PCA and CR opiate use. Preoperative opiate medication use was determined using patient records and interviews. Opioid tolerance was defined as the daily consumption of $30 \mathrm{mg}$ oral morphine or more.

RESULTS: Of the opiate naïve patients, 58 of 451 (12.9\%) required IV PCA, and CR opiates were administered in 35 of 451 (7.8\%). In the opioid tolerant patients, 28 of $49(57.1 \%)$ had IV PCA initiated, and 34 of $49(69.4 \%)$ received long acting opioids.

CONCLUSIONS: Given the extremely low requirement for IV PCA and $\mathrm{CR}$ opiate initiation in the opiate naïve patients observed, the data supports our institutional standard of using principally oral immediate release opiate therapy as part of a multimodal regimen. While valuable, the use of IV PCA and CR opiates may be avoided on a routine basis for opiate naïve patients from a safety standpoint. The opiate tolerant population may require increased flexibility in the initiation of these modalities.

\section{P11}

A RANDOMIZED CROSSOVER STUDY OF A

COMBINATION OF TARGIN ${ }^{\circledR}$ [OXYCODONE HCL AND NALOXONE HCL CONTROLLED-RELEASE (CR) TABLETS] AND CR OXYCODONE HCL TABLETS IN PATIENTS WITH CHRONIC NON-CANCER PAIN (CNCP) REQUIRING HIGH-DOSE OPIOIDS

P Arsenault ${ }^{1}$, J Balon ${ }^{2}$, P Bletcher ${ }^{3}$, G Chouinard ${ }^{4}$, C Cloutier ${ }^{5}$, M Donworth ${ }^{6}$, J Gillis ${ }^{7}, \mathrm{~S} \mathrm{Henein}^{8}$, A Kelly ${ }^{9}$, P MacDougall ${ }^{10}$, M Mille ${ }^{11}$, W O'Mahony ${ }^{12}$, P Rheault ${ }^{13}$, G Schacter ${ }^{14}$,

J Eisenhoffer ${ }^{15}$, Z Harsanyi ${ }^{15}$, K Michalko ${ }^{15}$, P Piraino ${ }^{15}$, P Sadri ${ }^{15}$

${ }^{1}$ Diex Recherche Sherbrooke Inc, Sherbrooke, Quebec; ${ }^{2} \mathrm{CPM} / \mathrm{AIM}$ Health Group, Trainyards, Ottawa; ${ }^{3}$ CPM Oshawa, Oshawa, Ontario; ${ }^{4}$ Recherche Clinique Sigma, Quebec City; ${ }^{5} \mathrm{CHUS}$ Hopital Fleurimont, Sherbrooke, Quebec; ${ }^{6} \mathrm{CPM} / \mathrm{AIM}$ Health Group, London, Ontario; ${ }^{7}$ CPM Dartmouth, Dartmouth, Nova Scotia; ${ }^{8}$ SKDS Research Inc, Newmarket, Ontario; ${ }^{9}$ Alberta Clinical Research Inc, Edmonton, Alberta; ${ }^{10} \mathrm{QEII}$ Health Sciences Centre, Halifax, Nova Scotia; ${ }^{11}$ Jarvis Family Medical Centre, Jarvis; ${ }^{12}$ Corunna Medical Research Centre, Corunna; ${ }^{13}$ Medicor Research Inc, Sudbury; ${ }^{14}$ London; ${ }^{15}$ Purdue Pharma, Pickering, Ontario AIM: To compare symptoms of opioid-induced constipation (OIC) and analgesic efficacy and safety of Targin ${ }^{\circledR}(\mathrm{OXN})$ to CR-oxycodone in a randomized, double-blind (DB), crossover study in CNCP subjects requiring high-dose opioids (60 $\mathrm{mg}$ or $80 \mathrm{mg}$ CR-oxycodone $\mathrm{q} 12 \mathrm{~h}$ )

METHODS: Subjects with CNCP $\geq 3$ months were enrolled. During a two-week run-in, the CR-oxycodone daily dose was optimized (moderate pain and $\leq 2$ rescue IR-oxycodone doses/day) to $60 \mathrm{mg}$ or $80 \mathrm{mg} \mathrm{q} 12 \mathrm{~h}$. Subjects who also had $<3$ complete spontaneous bowel movements (CSBM) in the last seven days of the Run-In were randomized. While receiving a static dose of CR-oxycodone, the 10-week DB period involved a crossover between stepped dose increases of naloxone or placebo, followed by a six-month open-label (OL) period.

RESULTS: The ITT population included 59 subjects. After five weeks of treatment, more subjects had $\geq 3$ CSBM on OXN than on CR oxycodone ( $29.5 \%$ versus $15.6 \% ; \mathrm{P}<0.0001)$. The mean VAS pain score at week 5 was equivalent for $\mathrm{OXN}$ and $\mathrm{CR}$-oxycodone $(54.9 \pm 18.7 \mathrm{~mm}$ versus $53.4 \pm 19.1 \mathrm{~mm} ;$ LS mean difference for CR-oxycodone $-\mathrm{OXN}=2.6[95 \%$ CI 0.2 to 5.4]). Improvements in bowel function and pain control were maintained throughout the OL period. The OXN AE profile was generally consistent with AEs reported at lower doses. The rate of withdrawal from treatment due to AEs was relatively low. No SAEs occurred during the DB period. Only one OL SAE (of six SAEs in five subjects) was deemed to have any causal relationship to study medication.

CONCLUSIONS: OXN $60 \mathrm{mg} / 30 \mathrm{mg}$ and $80 \mathrm{mg} / 40 \mathrm{mg}$ q12h significantly reduced the OIC burden without loss of analgesia in CNCP subjects

\section{P12}

DOES MORE PREPARATION FOR PARENTAL PRESENCE AT ANESTHESIA INDUCTION RESULT IN BETTER PEDIATRIC POST-OPERATIVE PAIN OUTCOMES?

Kristen Bailey ${ }^{1,2}$, Sally Bird ${ }^{3}$, Jessica MacKay ${ }^{4}$, Patrick McGrath $2,5,6,7,8,9$, Jill Chorney $2,9,10$

${ }^{1}$ Dalhousie University; ${ }^{2}$ Centre for Pediatric Pain Research; ${ }^{3}$ Department of Anesthesiology; ${ }^{4}$ Child Life Services, IWK Health Centre; ${ }^{5}$ Capital District Health Authority; ${ }^{6}$ Research \& Innovation, IWK Health Centre; ${ }^{7}$ Department of Pediatrics, ${ }^{8}$ Department of Psychiatry; ${ }^{9}$ Department of Psychology, Dalhousie University; ${ }^{10}$ Department of Anesthesiology, IWK Health Centre, Halifax, Nova Scotia

AIM: Preoperative anxiety is a significant predictor of postoperative pain and postoperative analgesic use (Kain, Mayes et al, 2006). Thus, managing children's preoperative anxiety is an important aspect of children's 
perioperative care. Parental presence during induction of anesthesia (PPIA) is a commonly used ways to help children manage their anxiety, but a recent Cochrane review concluded that PPIA did not change child or parent outcomes as compared to no PPIA (Yip et al, 2009). However, studies included in this review did not prepare parents to be in the operating room, which is thought to be related to children's preoperative anxiety (Kain et al, 2006; 2007). Objective: The purpose of this study is to compare the effectiveness of a brief video-based preparation intervention for PPIA (PPIA prep) to PPIA with typical logistical preparation (PPIA std).

METHODS: Participants \& Setting: The present study recruited 93 children (two to 10 years of age) scheduled to undergo elective surgery at the IWK Health Centre and their parents. Design \& Procedure: Parents were randomized to receive either PPIA prep or PPIA std just prior to their child's surgery. Children of parent's randomized to each group, were compared on measures of anxiety, pain, analgesic use and time required to recover from the surgery in hospital.

RESULTS: Children did not differ on measures of anxiety, pain or time to recover but children in the PPIA prep group had fewer doses of acetaminophen and had parents who felt more confident than children in the PPIA std group.

CONCLUSIONS: Parents may need more time and more support in order to learn and perform the behaviors they are instructed to perform in the intervention.

Footnotes/References:

Kain ZN, Caldwell-Andrews AA, Mayes LC, et al. Family-centered preparation for surgery improves perioperative outcomes in children: A randomized controlled trial. Anesthesiology 2007;106:65-74.

Kain ZN, Caldwell-Andrews AA, Maranets I, Nelson W, Mayes LC. Predicting which child-parent pair will benefit from parental presence during induction of anesthesia: A decision-making approach. Anesthesia and analgesia 2006;102:81-4.

Kain ZN, Mayes LC, Caldwell-Andrews AA, Karas DE, McClain, BC. Preoperative anxiety, postoperative pain, and behavioral recovery in young children undergoing surgery. Pediatrics 2006;118:651-8.

Yip P, Middleton P, Cyna A, Carlyle A. Non-pharmacological interventions for assisting the induction of anaesthesia in children (Review). The Cochrane Collaboration 2010(11):1-61.

\section{P13}

\section{SURVEY OF STAFF CONCERNS ABOUT PEDIATRIC PAIN MANAGEMENT}

\section{Breanna Balaton ${ }^{1}$, Krista Baerg ${ }^{2}$}

${ }^{1}$ Department of Anesthesiology, Perioperative Medicine and Pain Management; ${ }^{2}$ Department of Pediatrics, University of Saskatchewan, Saskatoon, Saskatchewan

AIM: Pain experienced by children impacts future pain experiences and changes neuroanatomical pathways for pain. Despite ethical considerations and improved knowledge about simple strategies to reduce distress related to painful procedures, pain is undermanaged. The aim of this survey is to identify types of patients and procedures where pediatric pain management is a concern for staff and their suggestions to improve pain management.

METHODS: Institutional ethics approval was obtained. The invitation to participate and 24-question anonymous survey were distributed to 1001 staff (July to August 2010) in ambulatory (including radiology and the test centre), inpatient, operative and emergency areas and to physicians and surgeons. Survey questions referred to observations of uncontrolled pain over the past month and experience with children from birth to 17 years of age. RESULTS: Two hundred and thirty five health professionals participated and $80 \%$ reported observing uncontrolled pain in a child over the month prior to the study period. Staff reported concern regarding intensity (32\%), duration $(25 \%)$ and frequency $(20 \%)$ of uncontrolled pain they observed in children. Only $60 \%$ of participants reported that pain is measured in their setting. Needle procedures such as blood draws and IV starts, fractures and dislocations, urethral catheterizations, spinal taps and nasogastric tube placements were most frequently identified by staff as procedures of concern. Surgical, trauma and pediatric medical patients were the most frequently identified patient populations of concern. Participants suggested policy or institutional guideline development, education for staff and parents, and improved access to resources to improve paediatric pain management.

CONCLUSIONS: Health professionals are concerned about the pain children experience with common procedures in acute care and ambulatory care settings. Many of these procedures can be managed with simple pharmacologic and nonpharmacologic methods. Future research is required to determine barriers to implementation of simple strategies known to reduce pain and improve coping. In keeping with suggestions from participants, evidence supports a multifaceted approach involving policy, education (parent and staff; ongoing), assessment, protocol development and self-monitoring to improve pain management.

\section{P14}

\section{EFFECT OF DIFFERENT VOLUMES OF EPIDURAL STEROID INJECTION ON PAIN RELIEF IN PATIENT RECIEVING FLUOROSCOPIC GUIDED INTERALAMINAR LUMBAR EPIDURAL STEROID INJECTION FOR LOW BACK PAIN}

Balbir Kumar, Jeetinder Kaur Makkar, Kajal Jain, YK Batra, Saravdeep S Dhutt

Post Graduate Institute Of Medical Education and Research, Chandigarh, India

AIM: We hypothesize that increase in volume of ESI has better effect on pain relief as an independent outcome. The primary outcome was to compare effect of different volumes of lumbar ESI on therapeutic efficacy ( $>50 \%$ pain relief) in LBP patients. The spread of iodinated contrast on fluoroscopy was the secondary outcome.

METHODS: Patients were randomized to receive $2 \mathrm{~mL}$ ( $80 \mathrm{mg}$ ) methylprednisolone acetate and $2 \mathrm{~mL} 2 \%$ lignocain in different volumes (by adding normal saline) into three groups: Group I, $4 \mathrm{~mL}$; group II, $6 \mathrm{~mL}$, group III, $8 \mathrm{~mL}$. Effective pain relief ( $\geq 50 \%$ from baseline) was evaluated by visual analogue scale (VAS) and improvement in disability by Modified Oswestry Disability Questionnaire (MODQ) at two and four weeks. Patients having $<50 \%$ pain relief received additional ESI of same dosage volume.

RESULTS: An interim analysis of patients with minimum one month follow up was performed to calculate sample size (18 patients analysed; group I, 6; group II, 7; group III, 5). Mean ( \pm SD) VAS scores at inception, two and four weeks were $9.0 \pm 0.632,4.33 \pm 2.33$ and $3.33 \pm 1.03$ in group I, $8.57 \pm 1.13,4.86 \pm 1.67$ and $3.57 \pm 0.78$ in group II, and $7.8 \pm 1.09$, $5.8 \pm 2.16$ and $4.4 \pm 2.3$ in group III, respectively. VAS were significantly lower at two and four weeks compared with baseline in three groups $(\mathrm{P}<0.05)$. Effective pain relief was $83.3 \%$ in group I, $57.1 \%$ in group II and $20 \%$ in group III at two weeks and $83.3 \%, 85.7 \%$ and $60 \%$ at four weeks, respectively. There was no significant decrease in MODQ.

CONCLUSIONS: Significant reduction in VAS underlines efficacy of ESI in LBP, but there was no statistically significant difference in effective pain relief achieved between three groups. However, this being an interim analysis further study with larger sample size is being carried out.

\section{P15}

PREDICTING SATISFACTION WITH SPOUSE RESPONSES AMONG PATIENTS WITH RHEUMATOID ARTHRITIS $\underline{\text { R Thomas Beggs }}{ }^{1}$, Susan Holtzman ${ }^{2}$, Anita DeLongis ${ }^{2}$ 1Department of Psychology, University of Guelph, Guelph, Ontario; 2Department of Psychology, University of British Columbia, Vancouver, British Columbia

AIM: A large body of literature has demonstrated that satisfaction with social support is associated with better emotional and physical adjustment to chronic pain. However, little is known about what causes a person to feel supported, and in particular, what might be responsible for withinperson fluctuations in satisfaction with support over time. The current study used a daily diary methodology to investigate factors associated with both within- and between-person variability in satisfaction with spouse responses.

METHODS: A total of 69 married patients with rheumatoid arthritis completed a background telephone interview, followed by twice-daily interviews for one week assessing pain intensity, positive and negative mood, specific spouse responses, and satisfaction with spouse responses. 
RESULTS: Results indicated that $>40 \%$ of the variance in satisfaction with spouse responses was within-patients. Multilevel modeling revealed that on mornings when patients reported esteem support (eg, spouse made them feel loved and accepted), they reported significant increases in satisfaction with support over the day. Although instrumental support (eg, spouse helped them take care of things) was associated with greater satisfaction, the effects did not last over the course of the day. Positive affect and lower pain were also associated with greater satisfaction, but only within the same timepoint. At the between-person level, older age, higher marital satisfaction, and lower fatigue were associated with greater satisfaction with spouse responses.

CONCLUSIONS: Findings suggest the potential clinical utility of encouraging the provision of esteem support among couples, and helps to identify RA patients who may be at risk for low satisfaction with painrelated support.

\section{P16}

"PAIN102" FOR TEENS WITH CHRONIC AND RECURRENT PAIN AND THEIR PARENTS: PRELIMINARY EVALUATION OF A MODIFIED VERSION OF PAIN 101

Susan M Bennett ${ }^{1,2,3}$, Erin C Moon ${ }^{1}$, Penny Sneddon ${ }^{1,2,3}$, Bruce D Dick $^{4,5}$, Anne-Claire Larochette ${ }^{6}$, Alexandra de Levie ${ }^{1}$ ${ }^{1}$ Department of Psychology, BC Childrens Hospital; ${ }^{2}$ Department of Psychology, University of British Columbia; ${ }^{3}$ Child and Family Research Institute, Vancouver, British Columbia; ${ }^{4}$ Departments of Anesthesiology and Pain Medicine, Psychiatry and Pediatrics, Multidisciplinary Pain Centre, University of Alberta; ${ }^{5}$ Pediatric Chronic Pain Clinic, Stollery Children's Hospital, Edmonton, Alberta; ${ }^{6}$ Department of Psychology, Queens University, Kingston, Ontario

AIM: This study explored the efficacy of a condensed and modified version of Pain101, a cognitive-behavioural group treatment program for teens with chronic and recurrent pain developed and evaluated at the University of Alberta.

METHODS: A four-session group program was implemented over a seven-week period at BC Children's Hospital. Sessions 1 and 4 involved both combined and concurrent programs for parents and teens. Sessions 2 and 3 involved teens only. Session content included: mind-body connection, goal-setting, sleep hygiene, pacing, cognitive-behavioural coping methods, parent-teen communication, and building a community care team. Session format included didactic, behavioural and experiential exercises.

Parent and teen self-report questionnaires consistent with those used at the University of Alberta were administered on first and last session days, and sent via mail at three months post-treatment. Outcome measures of pain, anxiety, depression, functional disability, sleep, quality of life, and hope were included.

Participants were recruited from the waitlist for outpatient psychology services. The 10 participants (seven female, three male) ranged from 13 to 17 years of age with chronic or recurrent pain. Each teen's mother, as well as one teen's father, also participated.

RESULTS: Data from sessions 1 and 4, as well as three month follow-up, have been collected to date. Preliminary results indicate non-significant trends by the end of session 4 , and significant improvements in teens' functional ability and depression by three months post-treatment. CONCLUSIONS: Preliminary results are consistent with outcome data from Pain101 (Dick et al, 2013). Longer-term follow up data (six and 12 months post-treatment) remain to be collected.

\section{P17}

\section{STAFF NURSE ADOPTION OF KANGAROO MOTHER CARE AS AN INTERVENTION FOR MANAGEMENT OF PROCEDURAL PAIN IN PRETERM INFANTS}

Britney Benoit ${ }^{1}$, Marsha Campbell-Yeo ${ }^{1,2,3,4}$, Celeste Johnston ${ }^{3,4}$, Margot Latimer ${ }^{1,2,3}$, Kim Caddell ${ }^{4}$, Talia Orr ${ }^{2}$

${ }^{1}$ Centre for Pediatric Pain Research, IWK Health Centre; ${ }^{2}$ School of Nursing, Dalhousie University; ${ }^{3}$ Department of Pediatrics;

${ }^{4}$ Maternal Newborn Program, IWK Health Centre, Halifax, Nova

Scotia

AIM: Skin-to-skin contact between mother and infant, commonly referred to as Kangaroo Mother Care (KMC), has demonstrated efficacy in reducing pain for a single procedure. Despite known benefits, and practice guidelines recommending $\mathrm{KMC}$ as a pain management strategy, this intervention remains underutilized. The purpose of this study was to examine nurse attitudes concerning the effectiveness, challenges, and adoption of $\mathrm{KMC}$ as an intervention for pain management in the Neonatal Intensive Care Unit (NICU).

METHODS: Nurses working in the NICU site of a trial examining the sustained efficacy of KMC were asked to complete an investigator developed questionnaire at two time points: one month and six months following trial initiation. Identified benefits, expectations, frequency of use, and challenges of KMC-related practice change were analyzed using descriptive statistics. Survey data from the two time points were compared using chisquare analysis.

RESULTS: Forty nurses completed surveys. Independent of group, 95\% of participants indicated they expected KMC to provide good or very good pain relief. Nurses reported less expected difficulty $\left(\chi^{2}=22.68 ; \mathrm{P}<0.001\right)$ and fewer concerns $\left(\chi^{2}=21.10 ; \mathrm{P}<0.001\right)$ regarding $\mathrm{KMC}$ for procedural pain between time points. However, the frequency of nurses who consistently used $\mathrm{KMC}$ as an intervention was $7.5 \%$, with no significant differences between the time points.

CONCLUSIONS: Despite positive expectations and reduced concerns related to $\mathrm{KMC}$ as a pain relieving intervention, frequency of $\mathrm{KMC}$ use remained unchanged. Research addressing ways to overcome contextual and systems related barriers to utilizing $\mathrm{KMC}$ as an intervention for procedural pain the NICU is warranted.

\section{P18}

WHAT MAKES A GOOD DISTRACTER? EXPLORING THE CHARACTERISTICS OF EFFECTIVE DISTRACTION INTERVENTIONS FOR NEEDLE-RELATED PAIN AND DISTRESS IN CHILDREN

Kathryn A Birnie ${ }^{1}$, Melanie Noel ${ }^{2}$, Jennifer A Parker ${ }^{3}$, Christine T Chambers ${ }^{1,3}$, Lindsay S Uman ${ }^{4}$, Steve R Kisely, Patrick J McGrath ${ }^{1,3}$

${ }^{1}$ Department of Psychology and Neuroscience, Dalhousie University, Halifax, Nova Scotia; ${ }^{2}$ Seattle Children's Research Institute, Seattle, Washington, USA; ${ }^{3}$ IWK Health Centre; ${ }^{4}$ Community Mental Health, IWK Health Centre, Halifax, Nova Scotia; ${ }^{5}$ School of Medicine, University of Queensland, Queensland, Australia

AIM: Distraction is the most studied and empirically supported psychological intervention for needle pain and distress in children. However, distraction interventions use very heterogeneous strategies and distracters, making it difficult to identify effective treatment components. This analysis explored characteristics of effective distraction interventions.

METHODS: Twenty-six randomized controlled trials of distraction were identified in a recent systematic review of needle-related pain and distress in children two to 19 years of age. Two authors independently coded all studies for four intervention characteristics identified in previous research as potentially relevant to treatment efficacy: adult versus no adult involvement, no/low versus high technology distracters, interactive versus passive distraction, and child choice versus no choice. Self-report, observer-report, and behavioural measures of pain and distress were examined. Chi-square tests of subgroup differences examined treatment efficacy based on distraction characteristics. 
RESULTS: Distraction effectively reduced self-reported pain irrespective of intervention characteristics. Interventions with no adult involvement or using passive distraction were marginally more efficacious in reducing selfreported distress than those with adult involvement $\left(\chi^{2}=3.31 ; \mathrm{P}=0.07\right)$ or interactive $\left(\chi^{2}=3.31 ; \mathrm{P}=0.07\right)$. Interactive interventions were marginally more efficacious in reducing observer-reported distress $\left(\chi^{2}=3.55 ; \mathrm{P}=0.06\right)$. No differences were observed based on the use of no/low versus high technology distracters or availability of child choice of distracters.

CONCLUSIONS: Findings support the robustness of distraction for reducing self-reported pain, regardless of distraction intervention characteristics. The degree of adult involvement and interactive nature of distraction may contribute to treatment efficacy; however, future studies directly comparing different distraction techniques are needed.

\section{P19}

PAIN MANAGEMENT CONTINUING EDUCATION ACTIVITY TAILORED FOR INFO-SANTÉ NURSES: CONTINUING EDUCATION NEEDS ASSESSMENT BY THE NOMINAL GROUP TECHNIQUE

Marc Boisvert, Patricia Bourgault, Frances Gallagher

École des sciences infirmières de l'Université de Sherbrooke, Sherbrooke, Quebec

AIM: Context: Approximately $68 \%$ of all calls at the Info-Santé nurse telephone service are for a health problem with a pain component. It is well recognised that pain is often poorly assessed by health care professionals. Considering the complexity of pain assessment by telephone and the many consequences of pain, it is essential that nurses in this service develop their pain management skills through continuing education. The goal of this study was to assess learnings needs of Info-Santé nurses regarding pain management before a continuing education activity.

METHODS: The nominal group technique was used to assess the nurses' pain management learning needs. Participants: The study took place in a Info-Santé call center in Sherbrooke (Québec). All the nurses of this center were eligible $(n=31)$. An opportunistic sample of 19 nurses participated to the study.

RESULTS: Through the nominal group technique sessions, the nurses prioritized some topics that relate to pain management. Each learning need has a global weighted score: Complete pain assessment (3.25), abdominal pain assessment (2.75), neurophysiology of pain (2.15), pain assessment of children and the elderly (2.15), non pharmacological treatment (2.00) and pharmacological treatment (1.50).

CONCLUSIONS: Nurses had various learning needs that were answered by a full day training and individual coaching sessions at the workplace. The effect of this tailored training activity on nurses' knowledge of pain and their practice regarding pain management were assessed through the study. Finally, all Info-Santé call centers of Québec are interested by this training.

\section{P20}

EVALUATION OF THE PRELIMINARY EFFECTIVENESS OF HAND-MASSAGE THERAPY ON POSTOPERATIVE PAIN OF CARDIAC SURGERY ADULTS IN THE INTENSIVE CARE UNIT

Madalina Boitor ${ }^{1}$, Geraldine Martorella ${ }^{1,2,3}$, Caroline Arbour ${ }^{1,3,4}$, Cécile Michaud $^{3}, 5$, Céline Gélinas ${ }^{1,3,4,6}$

${ }^{1}$ Ingram School of Nursing, McGill University; ${ }^{2}$ Faculty of Nursing, Université de Montréal; ${ }^{3}$ Quebec Nursing Interventions Research Network (RRISIQ); ${ }^{4}$ Alan Edwards Centre for Research on Pain, McGill University, Montreal; ${ }^{5}$ School of Nursing, Université de Sherbrooke, Sherbrooke; ${ }^{6}$ Centre for Nursing Research and Lady Davis Institute, Jewish General Hospital, Montreal, Quebec

AIM: This study aimed to evaluate the preliminary effectiveness of hand massage on postoperative pain in the ICU.

METHODS: A pilot randomized controlled trial (RCT) was conducted with 40 adult ICU patients post cardiac surgery. Patients were randomized to either experimental (EG) $(n=21)$ or control group (CG) $(n=19)$. The EG received a 15 min hand-massage with lavender cream followed by a 30 min rest period. The CG had the cream applied to their hands without massage followed by the rest period. All participants received at least two interventions (massage or control), and 12 of them, a third one from a trained nurse within the first $24 \mathrm{~h}$ postoperative period. Pain variables ( 0 to 10 intensity, 0 to 8 Critical-Care Pain Observation Tool for behaviors) were collected before and after each intervention. Participants were also interviewed within $24 \mathrm{~h}$ post-ICU discharge with the Brief Pain Inventory (BPI).

RESULTS: A trend towards significance for the second intervention $(\mathrm{P}=0.088)$, and a treatment effect for the third intervention $(\mathrm{F}[2,20]=6.30$; $\mathrm{P}=0.008)$ were found for the $\mathrm{EG}$ who reported a greater decrease in pain intensity post-massage. Significantly less pain behaviors (ie, lower CPOT scores) post-massage were found for the EG for the first intervention (Mann Whitney=83.5; $\mathrm{P}=0.047$ ). No significant results were obtained using the BPI.

CONCLUSIONS: This pilot RCT showed promising results supporting potential benefits of hand-massage as a non-pharmacological intervention for pain management in postoperative ICU patients. A larger RCT is warranted to further explore its effects on pain, but until then, this low-cost intervention can be safely administered.

\section{P21}

\section{POTENTIAL HEALTH BENEFITS OF SELF-DISCLOSURE IN A CHRONIC PAIN SELF-MANAGEMENT PROGRAM: A PILOT STUDY}

Mandy Bouchard ${ }^{1}$, Barbara Adams ${ }^{2}$, Lynne Robinson ${ }^{3}$

${ }^{1}$ Interdisciplinary PhD Program, Dalhousie University, Halifax;

${ }^{2}$ Atlantic Balance and Dizziness Centre, Dartmouth; ${ }^{3}$ Department of Health and Human Performance, Dalhousie University, Halifax, Nova Scotia

AIM: Psychosocial interventions, including self-management programs, are showing a clear role in the care of chronic pain patients and techniques such as self-disclosure show some evidence of health benefits. Exploring self-disclosure in a peer setting has the potential to be accessible, without long wait times, and extensive cost to the patient and may improve outcomes in chronic pain patients. This study examined a group self-management program with an experimental intervention, a distress-disclosure component, to encourage patients to process negative emotions as an additional aspect of multidisciplinary care.

METHODS: A prospective randomized experimental study was conducted using a sample of chronic pain patients from Halifax, NS. The sample consisted of $n=9$ in the treatment group and $n=10$ in the control group. Participants attended one session of a group out-patient physiotherapy self-management program every week for six weeks. The treatment group had an additional 30 min within each session, consisting of $15 \mathrm{~min}$ of one on one self-disclosure and 15 min of group self-disclosure.

RESULTS: Distress disclosure was found to be significantly higher in the treatment as compared to the control group post program. For both treatment and control groups, higher distress disclosure was associated with improved physical well-being and a higher pedometer count. Improvements in pedometer count in the control group and improvements in pain scores in the treatment group were found post program.

CONCLUSIONS: This study demonstrated that attending this selfmanagement program while increasing one's distress disclosure can potentially help to reduce the intensity of chronic pain. Further research is needed.

\section{P22}

POSTOP MODERATE PAIN IS STILL PREVALENT IN HOSPITALIZED ADULTS: CLAIMING FOR A MULTIMODAL ANALGESIC ALGORITHM

Patricia Bourgault ${ }^{1}$, Sandeep Mayer ${ }^{2}$, Johanne Lapré ${ }^{2}$, Robert Thiffault ${ }^{2}$, Marie-Andrée Lesage ${ }^{2}$, Christian Cloutier ${ }^{2}$, Sylvie Lafrenaye ${ }^{2}$

${ }^{1}$ Université de Sherbrooke; ${ }^{2}$ Centre hospitalier universitaire de Sherbrooke, Université de Sherbrooke, Sherbrooke, Quebec Multimodal approach combining different analgesics results in optimal pain relief (ANZCA, 2010). Since 2001 our institution has pioneered the 
use of a multimodal approach in pediatric pain management based on a nurse administered VAS algorithm (Falanga et al, 2006).

AIM: To describe the actual pain management state of our adult patient population.

METHODS: Patients undergoing elective surgery (general, urologic or vascular) were recruited. During the first two postop days, they completed a pain diary and rated mean pain at the end of each day. Analgesic medications were recorded.

RESULTS: A total of 152 patients completed at least two days of pain diary: analgesic regimen by usual care (UC) $(n=56)$, patient-controlled analgesia (PCA) $(n=54)$ and epidural (EPI) $(n=32)$. Patients under UC received gabapentin (16\%), NSAID (50\%), opioids (89\%) and acetaminophen $(91 \%)$. Medication pattern for PCA and EPI was similar except for higher use of gabapentin for patients using PCA (30\%) and EPI (34\%). Mean $( \pm$ SD) pain rating (maximum pain $=10$ ) on day 1 was $4.4 \pm 2.6$ for UC, $4.9 \pm 2.1$ for PCA and $4.2 \pm 2.9$ for EPI. Pain decreased on day 2 with a mean pain rating of $3.3 \pm 2.4$ for $\mathrm{UC}$ but stayed the same for PCA $(4.5 \pm 1.8)$ and EPI $(4.1 \pm 3.6)$.

CONCLUSIONS: In adult surgical patients, pain remains in the moderate range regardless of the analgesic regimen used. The next step of our study is to implement the multimodal algorithm. Based on these results, improvement on pain management is still of interest.

Footnotes/References: Falanga I, Lafrenaye S, Mayer SK, Tétreault JP. Management of acute pain in children: Safety and efficacy of a nurse-controlled algorithm for pain relief. Acute Pain 2006;8:45-54.

\section{P23}

\section{SOCIAL FUNCTIONING OF YOUNG ADULTS DIAGNOSED WITH JUVENILE-ONSET FIBROMYALGIA SYNDROME COMPARED TO HEALTHY CONTROLS: A SIX-YEAR FOLLOW-UP}

Maggie H Bromberg ${ }^{1}$, Soumitri Sil ${ }^{2}$, Natoshia Cunningham ${ }^{3}$, Anne Lynch Jordan ${ }^{3}$, Tracy V Ting ${ }^{3}$, Daniel Strotman ${ }^{3}$, James Peugh ${ }^{3}$, Susmita Kashikar-Zuck ${ }^{3}$

${ }^{1}$ Seattle Children's Research Institute, Seattle, Washington; ${ }^{2}$ Children's Healthcare of Atlanta, Atlanta, Georgia; ${ }^{3}$ Cincinnati Children's Hospital Medical Center, Cincinnati, Ohio, USA

AIM: Adolescents with juvenile-onset fibromyalgia (JFM) are more isolated, withdrawn, and less popular than healthy peers. It is unknown whether difficulties with social functioning persist among youth with JFM as they transition into young adulthood. As part of a prospective study, we examined developmentally salient social functioning six years later in young adults (YA) with JFM and healthy peers (HP), and the relationship of depressive symptoms and pain intensity to social functioning.

METHODS: Participants were individuals (current mean $[ \pm \mathrm{SD}]$ age 21.6 \pm 1.9$)$ diagnosed with JFM in their adolescent years $(n=94)$ and demographically matched HP $(n=33)$ participating in a longitudinal study of JFM. Participants completed electronic measures of current social ties (Social Network Inventory), general social functioning (SF-36 Social Functioning), social support (Medical Outcomes Study Social Support Survey, MOS), average pain intensity (0 to 10 scale), and depressive symptoms (Beck Depression Inventory).

RESULTS: YA with JFM reported lower SF-36 social functioning and lower MOS social support than peers ( $\mathrm{P}$ values $<0.05$ ) but no differences in number of social ties. Within the JFM group, higher depressive symptoms correlated with lower social support on MOS subscales and lower SF-36 social functioning; higher average pain intensity was only associated with lower SF-36 social functioning.

CONCLUSIONS: YA with JFM continue experiencing poorer social functioning than HP. Despite similar quantity of social contacts, perceived quality of social support and general social functioning were lower for YA with JFM and were associated with higher depressive symptoms. Social problems in adolescent-onset JFM persist into young adulthood, suggesting a need for early psychosocial assessment and intervention.

\section{P24} THE INFLUENCE OF CATASTROPHIZING ON EXPRESSION
AND SELF-PERCEPTION OF PAIN

Erin Browne, Kimberley Kaseweter, Kenneth Prkachin

University of Northern British Columbia, Prince George, British

Columbia

AIM: The present study investigated individual differences in communication of pain experience. Specifically, we examined the influence of pain catastrophizing on pain experience, pain expressiveness, and self-perceptual accuracy.

METHODS: 62 participants completed a covertly videotaped cold-pressor task, during which they made ratings of their pain experience (unpleasantness and intensity) using visual analogue scales. In a second session, participants completed the Pain Catastrophizing Scale (PCS) and viewed their own recorded facial expressions, rating their pain using the same VAS scales. An expert in facial coding also viewed each video, completed the VAS ratings, and used a modified version of FACS (Facial Action Coding System) to identify and score pain-related facial expressions.

RESULTS: PCS scores positively correlated with VAS ratings during the cold pressor, third-person ratings of pain intensity, and accuracy in selfperception of pain. PCS scores significantly predicted accuracy in selfperception of pain unpleasantness and intensity, accounting for $8 \%$ and $13 \%$ of variance, respectively. This effect remains significant with the inclusion of average FACS scores as a control variable, but does not with inclusion of average third-person pain ratings.

CONCLUSIONS: Evidence from this study suggests that individuals high in pain catastrophizing rate their pain experience higher, are more expressive during the experience, and perceive more accurately in the role of self-observer. This phenomenon is not explained by an increased presence of typical pain-related facial expressions. It seems that individuals high in catastrophizing effectively communicate a heightened pain experience using alternative behavioural cues visible to themselves and to thirdperson observers.

\section{P25}

\section{WHAT PEOPLE WITH CHRONIC PAIN WANT: STRATEGIES TO SUPPORT RESUMPTION OF WORK}

Danielle Burgsma, Duncan Boothby, Kristine Germain, Alison Senior, Lisa Klinger, Lynn Shaw

School of Occupational Therapy, Western University, London, Ontario

AIM: The purpose of the research was to discover the strategies and barriers that have enabled or impeded return to work from the perspectives of individuals who suffer from chronic pain.

METHODS: A metasynthesis of qualitative literature was conducted to identify available research offering direct quotes of persons living with chronic pain. A pragmatic approach guided the methodology. Articles from the database search were identified based on strict inclusion and exclusion criteria. Selected articles then underwent title, abstract and full article screens, as well as received a two-stage quality appraisal before being approved for data extraction and synthesis. Relevant quotes were extracted and synthesized to identify central themes at the conceptual level.

RESULTS: Eight articles underwent data extraction and synthesis, which exposed five themes for strategies for return to work, such as 'negotiating balance' and three themes for barriers to return to work, such as 'lack of accommodations'.

CONCLUSIONS: The return to work process is complex and the development of return to work strategies requires the participation of persons with chronic pain, as they can provide insights into the unique barriers or strategies that exist. Enabling greater participation of persons with chronic pain in strategy development and problem solving may also achieve better long-term success in coping with symptoms and limitations while participating in productive occupations. The results will be used to develop clinical best practice guidelines for occupational therapists assisting persons with chronic pain in the return to work process. 


\section{P26}

CHARACTERISTICS OF PATIENTS ON LONG-TERM OPIOID THERAPY FOR CHRONIC NON-CANCER PAIN: A SURVEY

Jason W Busse ${ }^{1}$, Hamza Mahmood ${ }^{1}$, Bilal Maqbool' ${ }^{1}$,

Amna Maqbool' ${ }^{1}$, Ali Zahran' ${ }^{1}$, Adnan Mohammed',

Eshaq Alshaqaq ${ }^{1}$, Lynn Cooper ${ }^{2}$, Angela Carol ${ }^{1}$, Janice Sumpton ${ }^{3}$,

Erin McGinnis ${ }^{4}$, Melinda J Fowler ${ }^{1}$, Daniel Rosenbaum ${ }^{1}$,

Natalie Lidster ${ }^{1}$, Norm Buckley ${ }^{1}$

${ }^{1}$ McMaster University, Hamilton; ${ }^{2}$ Canadian Pain Coalition;

${ }^{3}$ London Health Sciences Centre, London; ${ }^{4}$ Niagara Region Public Health, Thorold, Ontario

AIM: We surveyed patients attending a hospital-based chronic pain clinic to explore characteristics of patients pursuing long-term ( $>6$ months) opioid therapy for chronic non-cancer pain.

METHODS: We administered a 21 -item survey to 168 patients presenting to the McMaster University chronic pain clinic that inquired about demographic variables, pain relief, functional improvement, side-effects, and impressions regarding an educational pamphlet (Are You Thinking About Taking Opioids for Your Pain?).

RESULTS: Ninety-one percent of respondents were receiving long-term opioid therapy and, of these patients, $59 \%$ had been taking opioids for $>5$ years. Most patients prescribed long-term opioid therapy were female $(62 \%)$, the mean $( \pm$ SD) age was $53 \pm 13$ years, and $16 \%$ had completed a university degree. The most common complaint for which opioid therapy was prescribed was chronic low back pain (65\% of patients). Most patients were receiving disability benefits or involved in litigation (68\%) and only $3 \%$ were working full-time at unmodified duties. Greater pain relief was reported versus functional improvement; $40 \%$ reported $>60 \%$ pain relief, and $32 \%$ reported $>60 \%$ functional improvement. Forty-seven percent endorsed that side-effects associated with opioid use were problematic. With regards to the educational pamphlet, $13 \%$ were unaware of the information before staring long-term opioid use, and $17 \%$ felt it provided too little information.

CONCLUSIONS: Long-term opioid use is common among patients attending the McMaster chronic pain clinic. Adequate pain relief is more common than functional improvement, and despite long-term opioid use the large majority of patients remain unemployed.

\section{P27}

INDEPENDENT MEDICAL EVALUATIONS - IMPORTANT, NEGLECTED, IN NEED OF REFORM: A SYSTEMATIC REVIEW Jason W Busse ${ }^{1}$, Shanil Ebrahim ${ }^{1}$, John J Riva ${ }^{1}$, Sheena Bance ${ }^{2}$, Gordon H Guyatt ${ }^{1}$, Michael Bagby ${ }^{2}$, Regina Kunz ${ }^{3}$

${ }^{1}$ McMaster University, Hamilton; ${ }^{2}$ University of Toronto, Toronto, Ontario; ${ }^{3}$ University Hospital Basel, Basel, Switzerland

AIM: Independent medical evaluations (IMEs) are a common and influential form of assessment, often influencing whether patients receive compensation for an injury or illness. To inform the evidence-base underlying IMEs, we conducted a systematic review of all primary literature conducted in North America.

METHODS: We searched CINAHL, EMBASE, MEDLINE and PsycINFO and other sources for studies published through to Sept. 20, 2011. We included all primary literature on the topic of IMEs from a North American perspective. Assessment for study inclusion, data extraction and risk-of-bias analyses were performed in duplicate.

RESULTS: We included 52 studies, all of which were observational in design and most of which focussed on determining the rate of malingering among examinees. Estimates of non-credible symptom over-reporting among patients presenting for IMEs ranged from $16 \%$ to $56 \%$, with studies at lower risk of bias finding higher estimates that ranged from $41 \%$ to $56 \%$. Instruments used to detect malingering generally demonstrated high specificity but low sensitivity, which suggests that reliance on psychometric testing alone is likely to underreport the true prevalence of malingering. Inter-rater reliability among IME assessors was poor and examinees were largely dissatisfied with the assessment process and outcomes. Other studies found that inter-rater reliability among IME assessors for assigning degree of impairment to the same IME report was poor, and that patients presenting for an IME with external incentive (eg, litigation, disability benefits) perform systematically worse across a range of psychometric tests versus patients presenting with similar illness/injury but without external incentive.

CONCLUSIONS: Symptom exaggeration is common among patients presenting for IMEs, and particularly among those patients with external incentive. IME assessors reviewing the same case demonstrate little agreement regarding the degree of impairment that should be assigned. Standards for IME assessment and reporting are urgently needed to ensure greater reliability and validity of this common form of assessment.

\section{P28}

\section{PREDICTORS OF WORKERS COMPENSATION CLAIM DURATION AMONG WORKERS DISABLED DUE TO LOW BACK PAIN}

Jason W Busse ${ }^{1}$, Ivan Steenstra ${ }^{2}$, Shanil Ebrahim ${ }^{1}$, Diane Heels-Ansdell ${ }^{1}$, Stephen D Walter ${ }^{1}$, Gordon H Guyatt ${ }^{1}$ ${ }^{1}$ McMaster University, Hamilton; ${ }^{2}$ Institute for Work \& Health, Toronto, Ontario

AIM: Low back pain (LBP) is a common complaint among workers receiving Workers Compensation wage replacement benefits. We used the administrative data from the Ontario Workplace Safety and Insurance Board (WSIB) to explore the association between baseline characteristics and commonly reimbursed therapies and time to claim closure among workers disabled due to LBP.

METHODS: Using the WSIB administrative database, we acquired a random sample of 6,665 injured workers who reported an uncomplicated back injury (strain or sprain) with a date of injury between January 1 and June 30, 2005. We selected, a priori, 11 variables from the database that we judged may be associated with claim closure and predicted the direction of anticipated effects. We performed a time-to-event analysis using Cox proportional hazards regression to assess the association between time to claim closure and the independent variables. Receipt of WSIB-reimbursed chiropractic care or physiotherapy were treated as a time-dependent covariate to account for when treatment was initiated during the course of the disability claim.

RESULTS: Our adjusted regression analysis showed that older age and opioid prescription (adjusted HR 0.69 [99\% CI 0.53 to 0.89]) in the first four weeks of claim reimbursed by the WSIB were associated with prolonged claim closure, whereas working for an employer that had a returnto-work program was associated with shorter claim duration (adjusted HR 1.73 [99\% CI 1.42 to 2.12]). Neither reimbursement for chiropractic or physiotherapy were associated time to claim closure.

CONCLUSIONS: Our analysis suggests that commonly reimbursed treatment for Workers Compensation LBP claimants may be ineffective or even harmful.

\section{P29}

\section{PAIN OR GAIN? UNDERSTANDING THE RELATIONSHIP BETWEEN PAIN AND REWARDS IN CHILDREN AND ADOLESCENTS: A PILOT STUDY}

Line Caes ${ }^{1}$, David Ritcey ${ }^{1,2}$, Wiebke Gandhi ${ }^{3}$, Jennifer A Parker ${ }^{1}$, Petra Schweinhardt ${ }^{3}$, Christine T Chambers ${ }^{1,2,4,5}$

${ }^{1}$ Centre for Pediatric Pain Research, IWK Health Centre;

${ }^{2}$ Department of Pediatrics, Dalhousie University, Halifax; ${ }^{3}$ Alan Edwards Centre for Research on Pain \& Faculty of Dentistry, McGill University; ${ }^{4}$ Department of Psychology and Neurology; ${ }^{5}$ Department of Psychiatry, Dalhousie University, Halifax, Nova Scotia

AIM: Reward processes play an important role in understanding several health conditions but their role in pain experiences have only recently been investigated. In adults, increased motivation to obtain rewards in painful situations, without experiencing these rewards as more pleasant was observed. However, no research has investigated these pain-reward interactions in a pediatric sample. The purpose of the project is to modify a pain-reward paradigm successfully used in adults for use with children in which a computerized monetary reward task (MRT) is performed twice; once while performing the pain-inducing cold pressor task (CPT) and once 
with their hand in non-painful cold water. As the MRT takes 4 min to complete, a pilot study was deemed necessary to select a water temperature for the CPT that is considered painful but not too painful so that participants are unable to complete the MRT.

METHODS: Ten children (eight to 16 years of age) performed the CPT three times $\left(12^{\circ} \mathrm{C}, 15^{\circ} \mathrm{C}\right.$ and $25^{\circ} \mathrm{C}$ water) in a randomized order. During each CPT they completed a computerized reaction time task similar to the MRT but without receiving rewards.

RESULTS: No significant differences were found in the immersion time between $12^{\circ} \mathrm{C}$ versus $15^{\circ} \mathrm{C}$ water, but $12^{\circ} \mathrm{C}$ water was experienced as significantly more painful (M pain intensity $=5.2$ ). Water of $25^{\circ} \mathrm{C}$ was found to be not painful. Reaction times did not differ significantly for all three tasks. CONCLUSIONS: These results are valuable for the main project as they indicate that $12^{\circ} \mathrm{C}$ is the best water temperature to use in our adjusted pain-reward paradigm applicable in children.

\section{P30}

\section{IMPACT OF A QUALITY IMPROVEMENT STRATEGY FOR IMPROVING PAIN OUTCOMES IN A PEDIATRIC HOSPITAL SETTING}

Fiona Campbell ${ }^{1,2}$, Jennifer Peleshok ${ }^{1}$, Janet Yamada ${ }^{1,2}$, Anne-Marie Krancevic ${ }^{1}$, Jennifer Pepper ${ }^{1}$, Monica Dhaliwal ${ }^{1}$, Charles Victor $^{2}$, Jennifer Stinson ${ }^{1,2}$, Bonnie Stevens ${ }^{1,2}$

${ }^{1}$ The Hospital for Sick Children; ${ }^{2}$ University of Toronto, Toronto, Ontario

AIM: Pain is a priority indicator at The Hospital for Sick Children. Accordingly, a Quality Improvement Plan (QIP) was developed encompassing monitoring and feedback, reminders and skills development to improve organizational targets for inpatient pain satisfaction as captured by the National Research Corporation Canada (NRCC) survey.

METHODS: Monitoring and feedback: A paper-based audit tool was disseminated quarterly to capture clinical (ie, prevalence of moderate to severe pain) and process (frequency of pain assessments, interventions for mod-sev pain) outcomes over $24 \mathrm{~h}$ on 15 inpatient units. Feedback reports were generated comprising hospital-wide averages and unit outcomes.

Reminders and skills development: Those units with outcomes below hospital averages received customized reminders and web-based educational modules.

RESULTS: The QIP results indicated that frequency of pain assessments improved from 91\% (Q3; 2012/2013) to 99\% (Q2; 2013/2014). Patients with moderate to severe pain receiving any intervention fluctuated between 91\% (Q3; 2012/2013) and 86\% (Q2; 2013/2014). The percentage of patients with at least one moderate to severe pain intensity score fluctuated between 22\% (Q3; 2012/2013) and 26\% (Q3; 2013/2014). Positive trends in pain assessment frequency correlated with improved NRCC inpatient pain satisfaction: $80.67 \%$ (Q3; 2012/2013) to $85.5 \%$ (Q1; 2013/2014).

CONCLUSIONS: Implementation of the QIP has been successful in generating information regarding clinical and process outcomes, and is correlated with improved pain satisfaction among inpatients. We have shown improvements in pain assessment practices and, in future, will direct our attention towards pain prevention, and improved interventions for moderate to severe pain.

\section{P31}

AN INTEGRATIVE REVIEW OF BARRIERS TO EFFECTIVE PAIN MANAGEMENT: IDENTIFYING GAPS IN RESEARCH, POLICY, AND PRACTICE

Eloise Carr $^{1,2}$, Mia Ortiz $^{2}$, Stacy Dikareva ${ }^{2}$

${ }^{1}$ Faculty of Graduate Studies; ${ }^{2}$ Faculty of Nursing, University of Calgary, Calgary, Alberta

AIM: The purpose of this integrative literature review was to provide an analysis and synthesis of contemporary barriers to effective pain management.

METHODS: A search was performed using three electronic databases: CINAHL, PubMed, and Health Source (2003 to 2013). Databases were searched using terms: "nursing", "pain management", "pain education", and "barrier". Records were screened for: 1) original research articles whose primary or secondary research outcomes focused on barriers to effective pain management; and 2) published in the English language from 2003 to 2013. Included articles were then subjected to thematic analysis.

RESULTS: Twenty-four articles met inclusion criteria. Thematic analysis revealed three inter-related barriers: patient, professional, and organizational barriers. These barriers rarely occurred in isolation, with many studies reporting all three. Study methods included quantitative and qualitative approaches but lacked observational methods that provide insight into the complexities of clinical practice. Another gap to emerge related to a lack of public pain education.

CONCLUSIONS: To improve pain management, novel approaches, that address the multifactorial nature of pain management with a focus on knowledge translation, is required. Recommendations for future avenues of investigation include: 1) development of public health pain education initiatives that inform and facilitate patient engagement, 2) implementation of mandatory health professional undergraduate/pre-licensure pain education, and 3) focusing on workplace team cohesion through interprofessional and leadership education.

\section{P32}

\section{THE MEDIATING ROLE OF EXPECTANCIES ON THE RELATION BETWEEN DEPRESSION AND RETURN TO WORK OUTCOMES}

Junie S Carrière, Michael JL Sullivan

McGill University, Montreal, Quebec

AIM: To examine whether outcome expectancies mediate the relation between depression and return-to-work in individuals with work-related musculoskeletal disorders (WRMDs). WRMDs account for $50 \%$ of all time loss claims in Québec alone. Depressive symptoms have been identified as a significant risk factor for prolonged disability, however, little is known about the process by which depression impacts recovery following WRMDs.

METHODS: A sample of 164 patients with WRMDs were recruited from one of six physiotherapy clinics. Participants completed measures of pain severity, depression and return to work expectancies. Return to work status was assessed by telephone interview one year after the initial assessment.

RESULTS: Consistent with previous research, more severe depressive symptoms were associated with a lower probability of return to work. Regression analysis revealed that return to work expectancies completely mediated the relation between depression and return to work.

CONCLUSIONS: The results suggest that interventions specifically targeting outcome expectancies in individuals with WRMDs and depressive symptoms might improve return to work outcomes.

\section{P33}

\section{NEONATAL PAIN AND COMT RS4680 GENOTYPE IN RELATION TO SEROTONIN TRANSPORTER (SLC6A4) PROMOTER METHYLATION IN VERY PRETERM CHILDREN AT SCHOOL AGE}

Cecil MY Chau ${ }^{1}$, Manon Ranger ${ }^{1,2}$, Angela Devlin ${ }^{2,3}$, Tim F Oberlander ${ }^{1,2}$, Ruth E Grunau ${ }^{1,2}$

${ }^{1}$ Developmental Neurosciences \& Child Health, Child \& Family Research Institute; ${ }^{2}$ Pediatrics, University of British Columbia; ${ }^{3}$ Diabetes, Nutrition \& Metabolism, Child \& Family Research Institute, Vancouver, British Columbia

AIM: Children born very preterm are exposed to repeated neonatal procedural pain-related stress during hospitalization in the neonatal intensive care unit (NICU). The COMT rs4680 genotype is involved with pain sensitivity, and early life stress is implicated in altered methylation level of the serotonin transporter. We examined: (1) whether methylation of serotonin transporter (SLC6A4) promoter differs between very preterm children and full-term controls at school age, (2) relationships with child behavior problems, and (3) the extent of COMT rs4680 genotypes modulated the association between neonatal pain exposure and SLC6A4 methylation at seven years in the very preterm children.

METHODS: Participants comprised $n=111$ children, 61 born very preterm (24 to 32 weeks gestation), and 50 control children born full-term, all 
seen at mean age 7.8 years (SD 0.65 years). SLC6A4 and COMT rs4680 were genotyped from saliva DNA. SLC6A4 methylation was quantified by bisulfite pyrosequencing. Generalized linear modeling was used to examine associations between the COMT genotypes, neonatal pain exposure (adjusted for medical confounders), SLC6A4 methylation, and child behavioral problems.

RESULTS: Very preterm children had higher methylation at 7 of $10 \mathrm{CpG}$ sites in the SLC6A4 promoter compared to full-terms at age seven years. Greater neonatal pain (adjusted for medical confounders) was associated with higher Total child behavior problem score on the Child Behavior Checklist (CBCL) questionnaire (adjusted for concurrent stressors and 5HTTLPR genotype) $(\mathrm{P}=0.035)$. Higher $\mathrm{CBCL}$ total problem was associated with greater SLC6A4 methylation in very preterm children $(\mathrm{P}=0.01)$. In COMT Met/Met children, greater neonatal pain (adjusted for medical confounders) was associated with reduced methylation of SLC6A4 pro$\operatorname{moter}(\mathrm{P}=0.001)$.

CONCLUSIONS: We demonstrated a complex relationship between early exposure to highly stressful environmental events that induce repeated pain, child genotype reflecting pain sensitivity, and epigenetic modifications in children born during a critically sensitive developmental period. These findings provide evidence that both genetic predisposition and early environment need to be considered in understanding susceptibility for developing behavioral problems in this vulnerable population. Funding: Eunice Kennedy Shriver Institute of Child Health and Human Development RO1 HD039783

\section{P34}

PERIOPERATIVE GABAPENTIN REDUCES 24 H OPIOID CONSUMPTION AND IMPROVES IN-HOSPITAL REHABILITATION BUT NOT POST-DISCHARGE OUTCOMES FOLLOWING TOTAL KNEE ARTHROPLASTY

Hance Clarke $^{1}$, Joel Katz ${ }^{2}$, Colin McCartney ${ }^{3}$, Paul Stratford ${ }^{4}$, Deborah Kennedy ${ }^{4}$, M Gabrielle Pagé ${ }^{2}$, Imad T Awad ${ }^{3}$, Jeffrey Gollish ${ }^{5}$, Joseph Kay ${ }^{6}$

${ }^{1}$ Department of Anaesthesia, Toronto General Hospital; ${ }^{2}$ Department of Psychology, York University; ${ }^{3}$ Sunnybrook Health Sciences Centre, Toronto; ${ }^{4}$ School of Rehabilitation Science, McMaster University, Hamilton; ${ }^{5}$ Holland Orthopaedic and Arthritic Centre, Sunnybrook Health Sciences Centre, Toronto; ${ }^{6}$ Oakville Trafalgar Memorial Hospital, Oakville, Ontario

AIM: This study was designed to determine whether a four day perioperative regimen of gabapentin added to celecoxib improved in-hospital rehabilitation and physical function on postoperative day 4 , six weeks, and three months after total knee arthroplasty (TKA).

METHODS: After REB approval and informed consent, 184 patients were enrolled in a randomized, double-blind, placebo-controlled study. Two hours before surgery, patients received celecoxib $400 \mathrm{mg}$ po and were randomly assigned to receive either gabapentin $600 \mathrm{mg}$ po or placebo. Two hours later, patients received femoral, sciatic nerve blocks, and spinal anesthesia. Postoperatively, patients received gabapentin $200 \mathrm{mg}$ or placebo TID for four days. All patients also received celecoxib $200 \mathrm{mg}$ q12h for $72 \mathrm{~h}$ and i.v. PCA for $24 \mathrm{~h}$. Pain and function were assessed at baseline, during hospitalization, on postoperative day 4 , six weeks and three months after surgery.

RESULTS: The gabapentin group used less morphine in the first $24 \mathrm{~h}$ after surgery $(\mathrm{G}=38.3 \pm 29.5 \mathrm{mg}, \mathrm{P}=48.2 \pm 29.4 \mathrm{mg})$ and had increased knee range-of-motion compared to the placebo group in-hospital (both $\mathrm{P}<0.05)$. There were no differences between groups in pain or physical function on POD 4, six weeks or three months after TKA.

CONCLUSIONS: In the context of celecoxib, spinal anesthesia, femoral and sciatic nerve blocks, a dose of gabapentin $600 \mathrm{mg}$ preoperatively followed by four days of gabapentin $200 \mathrm{mg}$ TID decreased postoperative analgesic requirements and improved knee range of motion after TKA. Gabapentin provided no improvement in pain or physical function on postoperative day 4 , six weeks or three months following surgery.

\section{P35}

PREGABALIN REDUCES 24 H OPIOID CONSUMPTION AND IMPROVES DAILY PAIN FOR ONE WEEK AFTER DISCHARGE, BUT NOT FUNCTION AT SIX WEEKS, THREE MONTHS OR SIX MONTHS FOLLOWING TOTAL HIP ARTHROPLASTY

Hance Clarke $^{1,2}$, Colin McCartney ${ }^{3}$, Gabrielle Page ${ }^{4}$, Deborah Kennedy ${ }^{5}$, Paul Stratford ${ }^{5}$, Imad Awad ${ }^{3}$, Joseph Kay, ${ }^{6,7}$, Jeffrey Gollish ${ }^{3}$, Joel Katz ${ }^{1,2,4}$

${ }^{1}$ Department of Anesthesia and Pain Management, Toronto General Hospital; ${ }^{2}$ University Health Network; ${ }^{3}$ Sunnybrook Health Sciences Centre, Holland Orthopedic and Arthritic Centre; ${ }^{4}$ Department of Psychology, York University, Toronto; ${ }^{5}$ School of Rehabilitation Science, McMaster University, Hamilton; ${ }^{6}$ Oakville Trafalgar Memorial Hospital, Department of Anesthesia;

7McMaster University, Hamilton, Ontario

AIM: This study examined whether a perioperative regimen of pregabalin added to celecoxib improved pain scores and functional outcomes post discharge up to six months following total hip arthroplasty (THA).

METHODS: Following REB approval, 184 patients participated in a randomized, double-blind, placebo-controlled study. Two hours before receiving spinal anesthetic and undergoing surgery, patients received celecoxib $400 \mathrm{mg}$ po and were randomly assigned to receive either pregabalin (Prg) $150 \mathrm{mg}$ po or placebo (Pl). Postoperatively, patients received Prg75 mg or $\mathrm{Pl}$ in hospital and for one week following discharge. Patients also received celecoxib $200 \mathrm{mg} \mathrm{q} 12 \mathrm{~h}$ for $72 \mathrm{~h}$ and i.v. PCA for $24 \mathrm{~h}$. Pain and function were assessed at baseline, postoperative day 2 and 4 , six weeks, three months and six months (telephone follow-up) after surgery.

RESULTS: The pregabalin group used less morphine in the first $24 \mathrm{~h}$ postoperatively ( $\mathrm{Prg}, 39.85 \pm 28.1 \mathrm{mg} ; \mathrm{Pl}, 54.01 \pm 31.2 \mathrm{mg}$; $\mathrm{P}<0.01$ ). Pain scores were consistently lower in the pregabalin versus the control group every day to one week following discharge and the pregabalin group required less breakthrough opioid medications $(\mathrm{P}<0.05)$. There was no difference between groups on physical function or incidence and intensity of chronic pain at three and six months.

CONCLUSIONS: Perioperative pregabalin did not improve pain or physical function at six weeks, three or six months following THA. A postoperative pregabalin regimen decreased opioid consumption in-hospital, and following discharge improved daily pain scores and reduced adjunctive pain medications for seven days.

\section{P36}

THE DIFFERENTIAL ROLES OF OPTIMISM AND PESSIMISM IN YOUTH WITH CHRONIC PAIN

Laura Cousins $^{1}$, Lindsey Cohen ${ }^{1}$, Claudia Venable ${ }^{2}$

${ }^{1}$ Georgia State University; ${ }^{2}$ Children's Healthcare of Atlanta, Emory University School of Medicine, Atlanta, Georgia, USA

AIM: Optimism and pessimism are key constructs in predicting health outcomes; however, no studies to date have investigated these constructs in pediatric chronic pain. The current data compare the relations of optimism and pessimism with pain-related risk factors and functioning outcomes in a sample of children and adolescents with chronic pain.

METHODS: Participants included 58 children and adolescents with chronic pain who were between eight and 17 years of age (mean [ \pm SD] age $14.60 \pm 2.27$ years) and predominantly female $(n=46[79.3 \%])$. Participants completed questionnaires during an outpatient pain clinic visit. For regression analyses, pain intensity variables were controlled for in the first step and optimism and pessimism scores were entered simultaneously in the second step to predict fear of pain, catastrophizing, functional disability, and quality of life.

RESULTS: There was a negative correlation between children's optimism and pessimism $(\mathrm{r}=-0.773 ; \mathrm{P}<0.001)$. Although optimism and pessimism were not associated with average pain intensity, optimism was negatively correlated with worst pain intensity $(\mathrm{r}=-0.275 ; \mathrm{P}=038)$ and current pain intensity $(r=-0.259 ; \mathrm{P}=0.05)$. Regression analyses revealed that only optimism predicted quality of life, $\beta=0.599 ; \mathrm{P}=0.001$, and fear of pain, $\beta=-0.588, P=0.001$, and approached significance in predicting functional disability and pain catastrophizing (P values 0.08 to 0.09 ). 
CONCLUSIONS: Consistent with previous research in other pediatric populations, results indicate that optimism and pessimism are related but distinct constructs. Findings suggest that optimism - but not pessimism - is predictive of relevant clinical variables in pediatric chronic pain.

\section{P37}

IMPACT OF RESPONSIBILITY ATTRIBUTIONS AND PATIENT GENDER ON NURSES' AND NURSING STUDENTS' EMOTIONAL RESPONSES AND WILLINGNESS TO HELP PATIENTS WITH CHRONIC LOW BACK PAIN

$\underline{\text { L Crump }}^{1}$, DL LaChapelle${ }^{1}$, NC Higgins ${ }^{2}$, K Harman ${ }^{3}$, T Hadjistavropoulos ${ }^{4}$

${ }^{1}$ Department of Psychology, University of New Brunswick;

2Department of Psychology, St Thomas University, Fredericton, New Brunswick; ${ }^{3}$ School of Physiotherapy, Dalhousie University, Halifax, Nova Scotia; ${ }^{4}$ Department of Psychology, University of Regina, Regina, Saskatchewan

AIM: To evaluate Weiner's model of helping behaviour among nurses and nursing students making judgments about patients with low back pain (LBP). According to Weiner, attributions about patients' responsibility for their plight leads to emotional responses, which in turn contribute to helping behaviour.

METHODS: Observers ( 75 female nurses and 75 female nursing students) viewed videos of two male and two female patients performing a physiotherapy exercise. Accompanying patient descriptions indicated whether there was medical evidence of pathology and whether the patient used passive or active coping. Active copers with medical evidence were conceptualized as not responsible for their LBP while passive copers without medical evidence were conceptualized as responsible. Observers rated their feelings (anger, annoyance, sympathy) and willingness to help each patient.

RESULTS: There was only one significant main effect of responsibility: Observers felt more anger/annoyance towards patients deemed responsible. There were significant responsibility $\times$ observer effects for sympathy and helping behaviour: Nurses expressed more sympathy/willingness to help patients deemed not responsible but nursing students' sympathy/helping were not affected by responsibility. There were also significant responsibility $\times$ patient gender effects for sympathy and helping behaviour: Observers expressed more sympathy/willingness to help responsible male patients but non-responsible female patients. Moreover, sympathy (alone) significantly predicted helping regardless of gender or responsibility.

CONCLUSIONS: Weiner's model was supported: Responsibility predicted emotions, and sympathy was the only emotion that predicted helping. However, two potentially important moderators of these relationships were identified: patient gender and degree of nursing training.

Footnotes/References: Weiner B. A cognitive (attribution)-emotionaction model of motivated behavior: An analysis of judgments of helpgiving. J Pers Soc Psychol 1980;39:186.

\section{P38}

FACTORS RELATED TO PROCEDURAL PAIN MANAGEMENT IN NEONATAL INTENSIVE CARE UNITS: A SYSTEMATIC REVIEW

Maria Dulce Damas Cruz ${ }^{1}$, Ananda Maria Fernandes ${ }^{2}$, Catarina Resende de Oliveira ${ }^{3}$

${ }^{1}$ School of Nursing, University of Évora, Évora; ${ }^{2}$ Nursing School of Coimbra; ${ }^{3}$ Faculty of Medicine, University of Coimbra, Coimbra, Portugal

AIM: To identify associations between the frequencies of painful procedures performed in neonates and procedural pain management based on epidemiological observational studies.

METHODS: Electronic databases were searched (Medline, CINAHL, eLilacs, ScieLo) for primary studies focused on neonates until 28 days of age, admitted to neonatal or paediatric intensive care units, reporting the nature, frequency of three or more types of painful procedures and pain management interventions.

RESULTS: Twelve studies were included in this review. The selected studies were conducted between the years 1995 to 2012 in different countries.
In spite of the high frequency of procedures pain management is inconsistent. The frequency of procedures is associated with gestational age, first days of hospitalization, preterm neonates requiring mechanical ventilation, nasal oxygen and at the highest risk for neurological impairment. The use of specific pre-procedural analgesia was associated with prematurity, parental presence during procedures, daytime performance and day of hospitalization. Neonates with lower Clinical Risk Index for Babies scores, who were on high-frequency ventilation, who had undergone surgery, receive more pharmacologic interventions. The use of analgesia was also associated with the category of procedure, physician pain assessment, and for sweet taste or non-pharmacologic analgesia with parental presence. There was little relationship between protocols and clinical practice.

CONCLUSIONS: Preterm infants, neonates at high risk of neurologic impairment are more vulnerable to receive more painful procedures and less analgesia. Health professionals should be aware of the factors related to procedural pain management.

\section{P39}

PAIN TOLERANCE OF PARENTS AND CHILDREN DURING NEEDLE PROCEDURES

Jessica Dalley ${ }^{1}$, C Meghan McMurtry ${ }^{2}$, Patricia Creary ${ }^{2}$

${ }^{1}$ University of Guelph; ${ }^{2}$ Psychology, University of Guelph, Guelph, Ontario

AIM: To determine the level of pain parents and children report being able to tolerate during needle procedures before desiring pain management.

METHODS: Eighty-six parents and 79 children (five to 14 years of age; mean age 8.13 years) completed a questionnaire on needle fear and their tolerance for pain during needle procedures using 0 to 10 intensity scales. Adults also reported their beliefs regarding how much pain children could tolerate before requiring treatment.

RESULTS: While children report being able to tolerate a higher level of pain (mean $(4.32 \pm 0.38)$ during needles than adults expect $(3.28 \pm 0.19)$, $t(252)=-5.54, P=0.016, d=0.42$, children report needing treatment for moderate levels of pain and higher. 54\% of participants reported a current or previous fear of needles. For participants $>13$ years of age, as fear regarding needle procedures increased, their ability to tolerate pain without treatment decreased, $r s=-0.21, P=0.024$. Adults who reported higher pain tolerance also believed children could tolerate higher levels of pain before requiring treatment, $\mathrm{rs}=0.65, \mathrm{P}<0.001$.

CONCLUSIONS: Parents and children are motivated to ensure a child's pain is managed during needles, as both believe that children require treatment for moderate pain. Needle fear in adults and teenagers is associated with requiring pain management at lower levels of pain. Interventions aimed at reducing needle fear may be effective in improving a patient's pain tolerance. If parents have high pain tolerance, their children may be at risk for under management of pain.

\section{P40}

\section{WITHDRAWN}

\section{P41}

\section{URINARY DRUG SCREENING IN THE MANAGEMENT OF} PAIN: IS IT HELPFUL IN MANAGEMENT?

Marc Andre DEntrement ${ }^{1}$, Mary E Lynch ${ }^{2}$, Alexander J Clark ${ }^{2}$ ${ }^{1}$ Université de Sherbrooke, Sherbrooke, Quebec; ${ }^{2}$ Dalhousie University, Halifax, Nova Scotia

INTRODUCTION: Opioids are important tools in the management of pain but also pose a significant addiction risk in a subgroup of individuals. The National Opioid Use Guideline Group (NOUGG) guidelines acknowledge that "there is no compelling evidence to guide physicians on identifying patients with chronic non cancer pain (CNCP) who should have a urine drug screen (UDS) or how often".

AIM: To study the prevalence of unexpected UDS results in patients presenting to a chronic pain centre for an initial consultation and to interview the treating physicians regarding the clinical implications of an unexpected result. 
METHODS: A total of 594 new patients with CNCP were examined at a chronic pain centre in 2012/2013. The charts of all new patients were reviewed, a database containing the sex, age and UDS results was created. A short systematic interview was conducted with the treating physician when UDS results were incongruent with the self-report history.

RESULTS: The percentage of patients with at least one unexpected result was $5.49 \%$. The treating physicians found UDS testing helpful in $72.22 \%$ of these cases, and the result influenced patient care in $50 \%$ of unexpected results.

CONCLUSIONS: Approximately 5.49\% of UDS results are inconsistent with the patient's history and physicians found this information helpful in the majority of cases but only influenced care half the time. This information will be useful as clinicians and decision makers identify how best to use resources in care of people presenting with chronic pain.

\section{P42}

THE EPIDURAL PERFORMANCE IMPROVEMENT PROJECT RIDE WITH US ON THE EPIDURAL CAMPAIN: NO PAIN IS THE PATIENT'S GAIN

\section{Kristine Desjardine, Amanda Burcher}

\section{Queensway Carleton Hospital, Ottawa, Ontario}

AIM: For years, any patient who required an epidural for the management of their post-operative pain was cared for in a special 4-bed room. Bed flow and room availability became significant barriers to optimal post-operative pain management.

Surgery had a vision that all patients should have access to the most appropriate and effective method of post-operative analgesia, regardless of bed availability, patient flow or nursing assignment.

METHODS: Using Lean methodology, the front line staff were called upon to develop a current state map outlining the process of caring for a patient with an epidural.

During the map creation, the fundamental goal was to examine current processes through the eyes of the patient, with the aim of identifying opportunities to enhance their recovery after surgery. After making the step-bystep map, the staff then identified many ways in which care/processes could be streamlined.

A go live date was set for April 4, 2013 to launch the new processes.

RESULTS: Current best practices in post-operative pain management, staff feedback from surveys and the future state process map, several key changes were required to improve performance:

All registered staff were offered the opportunity to learn about epidurals and post-op pain management best practices

Requirement of Annual Certification-Refining nursing policies and procedures to support the change in practice

Leveraging an expert physician partner and revised the current medication orders to reflect current standards

Increases in equipment offering more epidural solutions from the Department of Pharmacy \& the ability to deliver a Registered Nurse Controlled Bolus Visual Cues were incorporated. Optimizing bedboard teletracking system to flag patients with an epidural

CONCLUSIONS: A future state map illustrating the steps involved in caring for a patient with an epidural post-operatively was created.This resulted in clinically redesigning processes on the unit and changing our care model of how patietns with an Epidural are managed. In the diagnostic phase data collection methods were used to analyze the results of the project which showed improvement. Epidural Metrics reporting tool was created and targets set based on the baseline data collected. metrics are reported monthly and the targets have been meet within the first three months of the Project.

\section{P43}

CAREGIVERS' ATTITUDES ABOUT PAIN MEDICATION FOR CHILDREN

Fernanda Nascimento Pereira Doca ${ }^{1}$, Áderson Luiz Costa Junior ${ }^{1}$, Allen Finley $^{2}$, Maria Beatriz Martins Linhares ${ }^{3}$

${ }^{1}$ Institute of Psychology, University of Brasília, Brasília, Brazil;

${ }^{2}$ Anesthesia and Psychology, Pediatric Pain Service, Centre for Pediatric Pain Research, Dalhousie University, Halifax, Nova Scotia; ${ }^{3}$ Ribeirão Preto Medical School, University of São Paulo, Ribeirão Preto, Brazil

AIM: The aim of the present study was to examine the caregivers' attitudes about using pain medication for pediatric pain treatment.

METHODS: The sample consisted of 291 family-caregivers (88\% mothers) and 142 health professionals (62 physicians/80 nurses), who care pediatric inpatients in six public hospitals (Brasília, capital of Brazil). The Medication Attitude Questionnaire -MAQ (Brazilian version) was applied in the caregivers.

RESULTS: The MAQ mean scores were $71( \pm 9)$ for family-caregivers and $66( \pm 12)$ for health professionals. Eight-six percent of family-caregivers and $65 \%$ of health professionals believed that children should be given pain medication as little as possible because of the side effects. Pain medication is addictive in the opinion of 79\% family-caregivers and $91 \%$ health professionals. Furthermore, $85 \%$ of health professionals disagreed with the affirmative that pain medication works the same no matter how often it is used. The comparison between groups of caregivers showed that familycaregivers presented more negative attitudes than health professionals ( $\mathrm{t}=9.4$; CI 3.9 to $7.9 ; \mathrm{P} \leq 0.001)$.

CONCLUSIONS: Both family-caregivers and health professionals groups showed negative attitudes about medication for pediatric pain relief. The negative attitudes are more pronounced in the parents than the health professionals. These attitudes could be a great barrier to the adequate pain management in vulnerable population of children.

\section{P44}

\section{PERCEPTIONS OF PEDIATRIC INPATIENTS' CAREGIVERS ABOUT PAIN EXPRESSION}

Fernanda Nascimento Pereira Doca ${ }^{1}$, Áderson Luiz Costa Junior ${ }^{1}$, Allen Finley $^{2}$, Maria Beatriz Martins Linhares ${ }^{3}$

${ }^{1}$ Institute of Psychology, University of Brasília, Brasília, Brazil; ${ }^{2}$ Anesthesia and Psychology, Pediatric Pain Service, Centre for Pediatric Pain Research, Dalhousie University, Halifax, Nova Scotia; ${ }^{3}$ Ribeirão Preto Medical School, University of São Paulo, Ribeirão Preto, Brazil

AIM: The aim of the present study was to examine the perceptions about pain expression in pediatric inpatients' caregivers.

METHODS: The sample was comprised of 291 family-caregivers (88\% mothers) and 143 health professionals (62 physicians/81 nurses), who care pediatric inpatients in six public hospitals (Brasília, capital of Brazil). The caregivers answered the Parental Pain Perception Expression-PPPE (Brazilian version).

RESULTS: The mean of PPPE score in family-caregivers was $42( \pm 6.7)$, which indicate misconceptions about pediatric pain expressions. Among them, $72 \%$ believed that children who are playing are not in pain, $57 \%$ believed that children exaggerate pain, and $24 \%$ considered that children feel less pain than adults. The mean of PPPE score in health professionals was $35( \pm 7.3)$. Among them, $96 \%$ believed that children in pain have trouble sleeping, $64 \%$ considered that children complain about pain to get attention, and $59 \%$ believed that children always express pain by crying or whining. Furthermore, $96 \%$ and $93 \%$ of family-caregivers and $95 \%$ and $97 \%$ of health professionals believed that untreated pain cause physical and psychological damage, respectively. The comparison between groups of caregivers showed statistically significant difference in the PPPE scores $(\mathrm{t}=9.4$; CI $5.3 ; \mathrm{P} \leq 0.001)$; the family-caregivers presented more misconceptions about children's pain expressions than the health professionals.

CONCLUSIONS: Despite the improvement in the scientific knowledge about pediatric pain, the caregivers still have misconceptions about children's pain expressions, which probably have a negative impact in identification and management of pain. 
P45

ASSESSING RISK OF BIAS IN CLINICAL TRIALS OF KANGAROO MOTHER CARE AS AN INTERVENTION FOR PROCEDURAL PAIN IN PRETERM INFANTS

Lustine Dol ${ }^{1}$, Britney Benoit ${ }^{1}$, Talia Orr ${ }^{2}$, Celeste Johnston ${ }^{1,3}$, Rebekah Zee ${ }^{4}$, Marsha Campbell-Yeo ${ }^{1,2,5,6}$

${ }^{1}$ Centre for Pediatric Pain Research, IWK Health Centre; ${ }^{2}$ School of Nursing, Dalhousie University, Halifax, Nova Scotia; ${ }^{3}$ McGill University, Montreal, Quebec; ${ }^{4}$ Faculty of Medicine, Dalhousie University; ${ }^{5}$ Department of Pediatrics, Dalhousie University and IWK Health Centre; ${ }^{6}$ Women's and Newborn Health Program, IWK Health Centre, Halifax, Nova Scotia

AIM: Clinical trials have the potential to overestimate treatment effect based on methodological limitations. Such limitations may exist in the areas of selection, performance, detection, attrition, and reporting. Trials with high or unclear risk of bias have been associated with exaggeration of treatment effects.

METHODS: As part of a recent Cochrane Review on Skin-to-Skin Care (SSC), Evidence Based Reviews, CENTRAL, MEDLINE, PubMed, Embase, CINAHL, Web of Science, LILACS, SciELO, PsychINFO, AMED, and Dissertation Abstracts International databases were searched. Nineteen studies meet the inclusion criteria. Using the Cochrane guidelines, we examined the risk of bias in: 1) sequence generation; 2) allocation concealment; 3) blinding of participants, personnel, assessors; 4) incomplete outcome data; 5) selective outcome reporting; and 6) other sources of bias. All trials were assigned a low, high, or unclear risk of bias for each category. Four reviewers scored each study independently, and an expert in methodology verified each score.

RESULTS: Out of 19 studies, low risk of bias was identified for: sequence generation $(\mathrm{n}=11)$, incomplete outcome data $(\mathrm{n}=15)$, reporting bias $(n=16)$, and other bias $(n=12)$. Only three categories had high risk of bias: blinding $(n=3)$, reporting bias $(n=1)$, and other bias $(n=2)$. The two areas most likely to have unclear bias were blinding $(n=11)$ and allocation concealment $(\mathrm{n}=11)$.

CONCLUSIONS: Overall, studies demonstrated a low risk of bias, suggesting conclusions accurately represent treatment effects. Strategies to minimize unclear risk of bias remain warranted, yet given the nature of the SSC intervention, blinding and concealment may be difficult to achieve.

\section{P46}

\section{A SYSTEMATIC REVIEW OF KNOWLEDGE TRANSLATION IN PEDIATRIC PAIN}

Michelle M Gagnon ${ }^{1}$, Thomas Hadjistavropoulos ${ }^{1}$, Amy Hampton ${ }^{1}$, Jennifer Stinson ${ }^{2}$

${ }^{1}$ University of Regina, Regina, Saskatchewan; ${ }^{2}$ The Hospital for Sick Children, Lawrence S Bloomberg Faculty of Nursing, University of Toronto, Toronto, Ontario

AIM: Pain is inadequately managed in pediatric populations across healthcare settings. Childhood pain leads to unnecessary suffering and other negative consequences. Although training programs to improve healthcare provider knowledge and skills have been developed and evaluated, clinical practices have not always kept pace with advancing knowledge. Our goal was to systematically review the literature in order to determine the effectiveness of existing knowledge translation (KT) programs targeting healthcare providers and factors influencing successful outcomes.

METHODS: Systematic searches of Medline, Web of Science, and CINAHL were undertaken. Primary inclusion criteria were: Englishlanguage articles focusing on KT directed to healthcare providers, primary focus on pediatric pain as well as consideration of process (knowledge changes) and/or clinical pain-related outcomes (pain intensity).

RESULTS: A total of 1658 abstracts were screened for inclusion with 47 articles retained. Across studies, KT approaches varied widely from reading materials to simulation training. Staff satisfaction was high across programs. Knowledge or practice changes resulted from many, but not all programs. Sufficient research on the long-term effects of pediatric pain KT programs is lacking.
CONCLUSIONS: KT programs vary in quality and impact. Although several successful programs have been developed, pediatric pain remains a significant concern. Evidence-based KT program implementation and a focus on sustainability of outcomes must be given greater consideration in the field of pediatric pain.

\section{P47}

METHODS OF CALCULATING CLINICAL SIGNIFICANCE IN PAIN RESEARCH

Samantha R Fashler, Erin Ross, Joel Katz

York University, Toronto, Ontario

AIM: The most popular way to determine treatment effectiveness in pain research is statistical significance, but this fails to capture the real-life impact of pain management. This paper describes current methods of calculating clinical significance in pain research.

METHODS: A range of treatment outcome measures were identified: statistical significance, minimum clinically significant score differences (MCSDs), effect sizes (eg, Cohen's d, Pearson's r, risk potency measures, number needed to treat), reliable change indices (RCIs; eg, GulliksenLord-Novik method, Edwards-Nunnally method, Hageman-Arrindel method), and hierarchical linear modeling (HLM).

RESULTS: MCSDs provide patient indices of how much change is considered to be subjectively significant. Effect sizes measure the magnitude or association between treatment and control conditions, but not the impact on the patient. Measures of risk potency provide indices of success and failure of a treatment, but most suffer from arbitrary cut-off values that may skew the interpretation of results. The RCI compares an individual's pretreatment and post-treatment scores to determine if a patient has moved from the dysfunctional to the functional range. Measures of RCI are limited to a maximum of two data points, whereas HLM provides more reliable estimates when three or more data points are available.

CONCLUSIONS: Statistical significance is not sufficient to draw conclusions regarding the patient-level efficacy of pain management strategies. Using statistical methods can lead to erroneous conclusions and poor treatment outcomes as a result. To assess pre- to post-treatment change, RCIs should be used for two data points and HLM for three or more data points.

\section{P50}

\section{EPIDEMIOLOGY OF PAIN IN A PORTUGUESE CHILDREN'S HOSPITAL}

Diana Catarina Ferreira de Campos, Maria João Caldeira Pais, Ananda Fernandes

\section{Nursing School of Coimbra, Coimbra, Portugal}

AIM: To determine pain prevalence, intensity and location in hospitalized children and the impact of pain on daily activities.

METHODS: A descriptive study was conducted in one of three Portuguese pediatric hospitals (108 beds). Demographic and clinical data were collected by interview. Children above five years old and parents were asked about current pain and worst pain in the last $24 \mathrm{~h}$. Pain was assessed with Faces Pain Scale - Revised or Numeric Rating Scale, by self or proxy report.

RESULTS: One hundred and fourteen children (mean age $8.04 \pm 5.65$ years) were hospitalized due to surgical or medical problems and $63 \mathrm{had}$ surgery. More than one-half (52.6\%) reported pain during the last $24 \mathrm{~h}$. Moderate to severe pain was reported by $90 \%$ of the patients (mean intensity $6.33 \pm 2.21$ ). They reported abdomen and legs as the most common locations of pain. Pain had impact on daily activities (38\% to 53\%). Sleep patterns alteration was the problem most frequently reported. Analgesic prescription was present in $64 \%$ of the children. Of those who were in pain, $17 \%$ had received no pain medication. Present pain was reported by $26 \%$ and of these, $57 \%$ reported moderate to severe pain (mean intensity 4.24 1.96$)$.

CONCLUSIONS: As reported in other similar studies, pain prevalence in hospitalized children is high. Some children had moderate or severe pain and not all children in pain received pain relief medication. Pain management practices should be revised. Children's response to pain medications deserves further research. 
P51

\section{WITHDRAWN}

\section{P52}

\section{WITHDRAWN}

\section{P53}

\section{LOWER SOCIOECONOMIC STATUS ASSOCIATES WITH INCREASED SYMPTOM SEVERITY AND FUNCTIONAL IMPAIRMENT IN FIBROMYALGIA}

Mary-Ann Fitzcharles ${ }^{1}$, Rampakakis $\mathrm{E}^{2}$, Ste-Marie PA ${ }^{3}$, Sampalis $\mathrm{JS}^{4}$, Shir $\mathrm{Y}^{1}$

${ }^{1}$ Montreal General Hospital; ${ }^{2}$ JSS Medical Research; ${ }^{3}$ University of Montreal; ${ }^{4}$ Jewish General Hospital, Montreal, Quebec

AIM: Persons with lower socioeconomic status (SES) have poorer health status for many medical conditions, but has never been reported for fibromyalgia (FM). The effect of education level, a validated measurement of SES, on disease severity in FM was examined.

METHODS: A prospective cohort of FM patients was stratified according to education level: high school or less (Group 1; n=99), college (Group 2; $\mathrm{n}=84$ ), university (Group 3; $\mathrm{n}=63$ ). Demographic and disease severity measures were examined. Between-group differences were assessed and linear regression was used to assess differences while adjusting for confounders.

RESULTS: There were no significant differences between groups for the following parameters: disease duration, current medication categories, and total number of medications per patient. Higher education was associated with greater use of alternative medicines $(\mathrm{P}<0.001)$ and alternative practitioner treatments $(\mathrm{P}=0.021)$. Lower education was associated with older age $(\mathrm{P}=0.039)$, previous drug abuse $(\mathrm{P}=0.016)$, current unemployment $(\mathrm{P}<0.001)$ and lower score for: patient global assessment (PGA) $(\mathrm{P}=0.019)$, Fibromyalgia Impact Questionnaire (FIQ) $(\mathrm{P}=0.002)$, Health Assessment Questionnaire (HAQ) $(\mathrm{P}=0.001)$, McGill Pain Questionnaire (MPQ) $(\mathrm{P}=0.026)$, Pain Disability Index (PDI) $(\mathrm{P}=0.031)$, and Pain Catastrophizing Scale (PCS), $(\mathrm{P}=0.015)$, and remained significant when adjusting for age and gender differences. Pain severity, anxiety, and depression did not differ between groups.

CONCLUSIONS: FM patients with lower SES reported greater symptom severity, functional impairment and unemployment, but not mood disorder. Although FM spans all socioeconomic groups, societal factors, rather than specific disease characteristics or mental status, appear to play an important role in patients' perception of illness.

\section{P54}

RHEUMATOLOGISTS LACK CONFIDENCE IN KNOWLEDGE OF CANNABINOIDS IN THE MANAGEMENT OF RHEUMATIC CONDITIONS: A NEEDS ASSESSMENT OF CANADIAN RHEUMATOLOGISTS

Mary-Ann Fitzcharles ${ }^{1}$, Peter A Ste-Marie ${ }^{2}$, Daniel J Clauw ${ }^{3}$, Shahin Jamal ${ }^{4}$, Jacob Karsh ${ }^{5}$, Sharon LeClercq ${ }^{6}$, Jason J McDougall', Yoram Shir ${ }^{1}$, Kam Shojania ${ }^{4}$, Zach Walsh ${ }^{4}$

${ }^{1}$ Montreal General Hospital; ${ }^{2}$ University of Montreal, Montreal, Quebec; ${ }^{3}$ University of Michigan Medical Center, Ann Arbor, Michigan, USA; ${ }^{4}$ University of British Columbia, Vancouver, British Columbia; ${ }^{5}$ The Ottawa Hospital, Ottawa, Ontario; ${ }^{6}$ University of Calgary, Calgary, Alberta; ${ }^{7}$ Dalhousie University, Halifax, Nova Scotia

AIM: Pharmacologic treatment of chronic rheumatic pain is sub-optimal, leading patients to seek alternate treatments. Up to two-thirds of persons using medicinal herbal cannabis report rheumatic pain. We assessed rheumatologists' self-reported confidence in their knowledge of therapeutic cannabinoids.

METHODS: A 19-question survey was sent to the entire Canadian Rheumatology Association membership to examine confidence in: 1) knowledge of cannabinoids 2) perceived competence to counsel patients regarding cannabinoids in general and herbal cannabis specifically.
RESULTS: 128 (25\%) of 510 members responded to questions pertinent to rheumatology practice: $75 \%$ lacked confidence in cannabinoid knowledge, $45 \%$ believed no role for any cannabinoid, $70 \%$ no role for medical cannabis, $13 \%$ had previously recommended marihuana, 90\% lacked confidence to prescribe medical cannabis. When grouped according to cannabinoid knowledge confidence, "Confident" $(\mathrm{n}=33$ ) versus "Not-Confident" $(n=95)$, the following were reported: current role for medical cannabis $48 \%$ versus $23 \%$; previous prescription of pharmacological cannabinoid $33 \%$ versus $12 \%$; previous recommendation for medical cannabis $27 \%$ versus $7 \%$; never recommended either $39 \%$ versus $81 \%$; would not recommend either in future $33 \%$ versus $67 \% .33 \%$ of "Confident" group reported competence in prescribing medical cannabis. Concerns about risks of marihuana were in line with current literature.

CONCLUSIONS: Rheumatologists lack confidence in their knowledge of cannabinoids, and doubt their competence to prescribe cannabinoids, especially herbal cannabis. There is a disconnect between patients' needs, policy makers and physician ability to provide competent patient care. Guidance is required to inform rheumatologists on the prevailing evidence for the safe and effective use of cannabinoids.

\section{P55}

TO BEFRIEND OR NOT: NATURALLY DEVELOPING FRIENDSHIPS AMONG A GROUP OF ADOLESCENTS WITH CHRONIC PAIN

$\underline{\text { Paula A Forgeron }}^{1}$, Jill Chorney ${ }^{2}$, Tori Carlson ${ }^{3}$, Erica Plante ${ }^{1}$, Bruce Dick ${ }^{4}$

${ }^{1}$ School of Nursing University of Ottawa, Ottawa, Ontario; ${ }^{2}$ IWK Health Centre, Dalhousie University, Halifax, Nova Scotia; ${ }^{3}$ Alberta Children's Hospital, Calgary; ${ }^{4}$ University of Alberta, Stollery Children's Hospital, Edmonton, Alberta

AIM: To describe the frequency and characteristics of naturally occurring peer friendships among a group of adolescents with chronic pain and reasons for and against interest in befriending another.

METHODS: Adolescents with chronic pain who had attended a 10-week self-management program from three sites were invited to participate. An online survey captured internalizing behaviors, pain-related data, and perceived support from friends using standardized measures. Study-specific questions captured data on befriending another with chronic pain and specifics about these friendship interactions.

RESULTS: Forty participants completed the survey. Ninety-five percent (38) were girls; $32 \%$ (13) befriended another; $52 \%$ (21) were interested in befriending another; $15 \%$ (6) were not. Over half (62\%) of the friendships lasted at least one year (8), 38\% lasted under six months, and only two intermingled these with their regular friendships. Pain was discussed frequently during interactions. The most common reasons for not forming friendships were no time to exchange contact information during group and nothing in common with others. Reason for not being interested in forming a friendship also included nothing in common apart from pain. CONCLUSIONS: The majority of adolescents with chronic pain were interested in befriending another. Emotional support by feeling understood, encouraging each other, and discussing pain without fear that the other is disinterested was the main peer support provided. However, without common interests, this form of friendship is unlikely to last and is at risk for being overly solicitous by focusing on pain. It remains unclear whether the benefits of peer support translate into improved function or decreased loneliness.

\section{P56}

FUNCTION AND QUALITY OF LIFE AFTER LONG-TERM USE OF OPIOIDS FOR THE TREATMENT OF CHRONIC NON-CANCER PAIN (CNCP): A SYSTEMATIC REVIEW

Andrea Furlan 1,2,3, Emma Irvin'1, Dwayne Van Eerd ${ }^{1}$,

Nancy Carnide $^{1}$, Claire Munhall ${ }^{1}$, Melanie Fortune ${ }^{1}$, Jaemin Kim ${ }^{1}$, Norm Buckley ${ }^{4}$

${ }^{1}$ Institute for Work \& Health, ${ }^{2}$ Toronto Rehabilitation Institute, ${ }^{3}$ University of Toronto, Toronto; ${ }^{4}$ McMaster University, Hamilton, Ontario

AIM: While there is evidence from previous reviews of the short-term effectiveness of opioids for treating CNCP, little is still known about the 
long-term effects of opioids for this condition. The aim of this study was to conduct a systematic review of the published scientific literature of the impact of long-term opioid therapy on function and quality of life (QoL) among patients with CNCP.

METHODS: A systematic search was conducted in Medline, EMBASE, CINAHL, PsycINFO, Central and Business Source Premier. References were screened for relevance by the review team to identify studies with the population of interest (non-cancer pain greater than three months) relevant intervention (opioid use longer than three months), and with function and QoL outcomes. Both experimental and observational studies were considered. The quality of included studies was assessed and their findings extracted using standardized quality appraisal and data extraction forms. Relevancy screening, quality appraisal and data extraction were conducted independently by two reviewers and conflicting responses were resolved RESULTS: The initial search retrieved 16,288 references. We extracted data from 30 studies with sufficient detail for evidence synthesis. Extracted data from each study were synthesized by study design. Effects on function and QoL outcomes were summarized by comparing pre/post or intervention versus control group estimates.

CONCLUSIONS: Preliminary findings from randomized controlled trials showed function and QoL of patients improved or did not change after long-term opioid use. There is some evidence from observational studies that the same outcomes deteriorated.

\section{P57}

\section{A SYSTEMATIC REVIEW OF KNOWLEDGE TRANSLATION IN PEDIATRIC PAIN}

Michelle M Gagnon ${ }^{1}$, Thomas Hadjistavropoulos ${ }^{1}$, Amy Hampton ${ }^{1}$, Jennifer Stinson ${ }^{2}$

${ }^{1}$ University of Regina, Regina, Saskatchewan; ${ }^{2}$ The Hospital for Sick Children, Lawrence S Bloomberg Faculty of Nursing, University of Toronto, Toronto, Ontario

AIM: Pain is inadequately managed in pediatric populations across healthcare settings. Childhood pain leads to unnecessary suffering and other negative consequences. Although training programs to improve healthcare provider knowledge and skills have been developed and evaluated, clinical practices have not always kept pace with advancing knowledge. Our goal was to systematically review the literature in order to determine the effectiveness of existing knowledge translation (KT) programs targeting healthcare providers and factors influencing successful outcomes.

METHODS: Systematic searches of Medline, Web of Science, and CINAHL were undertaken. Primary inclusion criteria were: Englishlanguage articles focusing on $\mathrm{KT}$ directed to healthcare providers, primary focus on pediatric pain as well as consideration of process (knowledge changes) and/or clinical pain-related outcomes (pain intensity).

RESULTS: A total of 1658 abstracts were screened for inclusion with 47 articles retained. Across studies, KT approaches varied widely from reading materials to simulation training. Staff satisfaction was high across programs. Knowledge or practice changes resulted from many, but not all programs. Sufficient research on the long-term effects of pediatric pain KT programs is lacking.

CONCLUSIONS: KT programs vary in quality and impact. Although several successful programs have been developed, pediatric pain remains a significant concern. Evidence-based KT program implementation and a focus on sustainability of outcomes must be given greater consideration in the field of pediatric pain.

\section{P58}

AGE-RELATED PATTERNS IN THE MULTIDIMENSIONAL EXPERIENCE OF CANCER PAIN

Lynn R Gauthier ${ }^{1,2}$, Robert H Dworkin ${ }^{3}$, Camilla Zimmermann ${ }^{2,4}$, Gary Rodin ${ }^{2,4}$, David Warr ${ }^{2,4}$, S Lawrence Librach ${ }^{4,5}$,

Malcolm Moore ${ }^{2,4}$, Frances A Shepherd ${ }^{2,4}$, Rebecca Pillai Riddell ${ }^{1,6}$, Alison Macpherson ${ }^{1}$, Ronald Melzack ${ }^{7}$, Lucia Gagliese ${ }^{1,2,4}$

${ }^{1}$ York University; ${ }^{2}$ University Health Network, Toronto, Ontario;

${ }^{3}$ University of Rochester, Rochester, New York, USA; ${ }^{4}$ University of Toronto; ${ }^{5}$ Mount Sinai Hospital; ${ }^{6}$ Hospital for Sick Children, Toronto, Ontario; ${ }^{7}$ McGill University, Montreal, Quebec

AIM: Although many older cancer patients experience pain, our understanding of age-related patterns in cancer pain remains limited. The aim of this study was to identify unique and shared risk factors for cancer pain in older and younger patients.

METHODS: A total of 105 older (68.3 \pm 6.6 years of age) and 139 younger $(49.8 \pm 7.2$ years of age) patients with advanced cancer and pain, matched for sex and tumor group, completed measures of pain, and physical and psychosocial wellbeing. Demographic and clinical information was collected.

RESULTS: There were no age differences in pain intensity or pain qualities $(\mathrm{P} \geq 0.11)$. Backward multivariate linear regression identified significant $(\mathrm{P} \leq 0.05)$ correlates of pain intensity and pain qualities in older and younger patients, separately. In older and younger patients, pain intensity was associated with cancer treatment $(\beta=-0.40 ; \beta=-0.21)$, symptom severity $(\beta=0.19 ; \beta=0.22)$ and pain anxiety $(\beta=0.15 ; \beta=-0.19)$. It was also associated with functional status $(\beta=-0.20)$ in older patients, and comorbidity $(\beta=0.16)$, pain willingness $(\beta=-0.26)$ and intrusive thoughts $(\beta=0.09)$ in younger patients. Pain qualities were associated with symptom severity $(\beta=0.35)$ and pain willingness $(\beta=-0.23)$ in older patients, and comorbidity $(\beta=0.22)$, functional status $(\beta=-0.21)$, physical health quality of life (QOL; $\beta=-0.21)$, intrusive thoughts $(\beta=0.26)$ and catastrophizing $(\beta=0.18)$ in younger patients.

CONCLUSIONS: Several risk factors for cancer pain were the same across age groups, including treatment, functional status, symptom severity, pain willingness, and pain anxiety. Unique risks factors in younger patients included comorbidities, physical health QOL, intrusive thoughts, and catastrophizing, suggesting important age-related patterns that could inform the development of age-tailored treatments.

\section{P59}

\section{RESPITE WORKERS' OPINIONS ON PAIN IN CHILDREN WITH COGNITIVE IMPAIRMENTS: SENSATION, REACTION AND COMMUNICATION}

Lara Genik' ${ }^{1}$, C Meghan McMurtry1, Lynn Breau ${ }^{2}$

${ }^{1}$ University of Guelph, Guelph, Ontario; ${ }^{2}$ Glenrose Rehabilitation Hospital, Edmonton, Alberta

AIM: To compare pain-related beliefs of respite workers (RW) working directly with children with cognitive impairments (CI) to a sample of undergraduate students (UG) with minimal experience with children with CI.

METHODS: Participants were 217 UG (mean age 19.63 years), and 56 RW (mean age 33.37 years) who responded to the Pain Opinion Questionnaire measuring pain beliefs regarding children with CI versus 'typically developing' children (Breau et al, 2003) as part of a larger study. RESULTS: Results included a significant interaction between participant groups and perceptions regarding pain sensation in children with $\mathrm{CI}$, $\mathrm{F}(1.61,417.98)=3.43, \mathrm{P}=0.04$. Specifically, compared to UG, RW believed that a higher percentage of children with severe CI sense less pain than 'typically developing' children, $t(262)=2.63, P=0.009$. A significant interaction was also found between participant groups and their perceptions regarding children with Cl's emotional reaction to pain, $\mathrm{F}(1.66,429.20)=3.55, \mathrm{P}=0.04$. Compared to $\mathrm{UG}, \mathrm{RW}$ believed that a higher percentage of children with both moderate, $\mathrm{t}(263)=2.08, \mathrm{P}=0.04$, and severe $\mathrm{CI}, \mathrm{t}(261)=2.51, \mathrm{P}=0.01$, demonstrate a lower emotional reaction to pain than 'typically developing' children.

CONCLUSIONS: Compared to UG, RW believe a larger percentage of children with CI sense less pain and demonstrate lower emotional 
reactions to pain; these beliefs may not accurately reflect pain experience in children with CI. These beliefs could impact pain assessment and management in respite care settings, and ultimately, children's health-related quality of life.

Footnotes/References: Breau LM, MacLaren J, McGrath PJ, Camfield CS, Finley AF. Caregivers' beliefs regarding pain in children with cognitive impairment: Relation between pain sensation and reaction increases with severity of impairment. Clin J Pain 2003;19:335-44.

\section{P60}

A PROSPECTIVE, RANDOMIZED, DOUBLE-BLIND CLINICAL TRIAL COMPARING BUPIVACAINE VERSUS SALINE FOR LUMBAR TRIGGER POINT INJECTION THERAPY

Hili Giladi ${ }^{1}$, Louise Lamb ${ }^{1}$, Paul Etheridge ${ }^{1}$, Xianming Tan ${ }^{2}$, Yoram Shir ${ }^{1}$

${ }^{1}$ The Alan Edwards Pain Management Unit, McGill University Health Centre; ${ }^{2}$ Biostatistics Core Facility, McGill University Health Center, Montreal, Quebec

AIM: To examine the effects of lumbar myofascial trigger point injection with either local anesthetic or saline in patients with chronic low back pain (CLBP).

METHODS: This was a single-centre, parallel group, prospective, randomized, double-blind clinical trial of patients diagnosed with myofascial low back pain. Patients were assigned for monthly trigger point injections with either bupivacaine or normal saline during a four month period. Repeated pressure algometery measurements and health and wellbeing questionnaires were applied to evaluate the outcome of the treatment.

RESULTS: Forty-three patients were included in the final analysis, of which 22 were treated with bupivacaine and 21 with saline. The two groups showed comparable outcomes of pain intensity and wellbeing indices for their low back pain at the end of the study. Overall, patients who receiving bupivacaine did not report a benefit at the end of the study compared to patients receiving saline. However, when comparing the repeated outcomes from all visits, patients receiving saline showed less pain compared to patients receiving bupivacaine $(\mathrm{P}=0.06)$. A trend over time was found towards decrease in the number of trigger points $(\mathrm{P}=0.004)$ and towards increase in their median pressure algometry measurements $(\mathrm{P}=0.07)$ in both arms.

CONCLUSIONS: In patients suffering from myofascial CLBP, there is no meaningful clinical advantage in performing trigger point injections using either bupivacaine or saline. Further studies are warranted to evaluate the clinical benefit of this procedure in specific sub-populations of patients with CLBP.

\section{P61 \\ DEPRESSIVE SYMPTOMS PREDICT UNEMPLOYMENT STATUS ACROSS VARIOUS CHRONIC PAIN DIAGNOSES Hili Giladi $^{1}$, Whitney Scott ${ }^{2}$, Yoram Shir ${ }^{1}$, Michael JL Sullivan ${ }^{2}$ ${ }^{1}$ The Alan Edwards Pain Management Unit, McGill University Health Centre; ${ }^{2}$ Department of Psychology, McGill University, Montreal, Quebec}

AIM: No previous study has systematically examined whether depressive symptoms predict employment status in patients with chronic pain of various aetiologies. The purpose of this study was to examine the relationship between depressive symptoms and unemployment across a range of common chronic pain diagnoses.

METHODS: Subjects were recruited from the Quebec Pain Registry (QPR), a registry of chronic pain patients, which contains data from three tertiary pain centers in Quebec. Patients were grouped into the following diagnostic categories based on their primary pain diagnosis recorded by the QPR: low back pain (LBP); non-LBP musculoskeletal pain; myofascial pain; neuropathic pain; and visceral pain. Statistical analyses examined the associations between patient demographics, pain intensity, depressive symptoms, and employment status within each diagnostic group.

RESULTS: Of 3478 screened patients, 2382 were found eligible. The prevalence of pain diagnoses was: non-LBP musculoskeletal pain $-29.4 \%$, LBP $-29.3 \%$, neuropathic pain $-23.1 \%$, myofascial pain $-12.7 \%$ and visceral pain $-5.4 \%$. Depressive symptomatology was the only significant unique predictor of unemployment status across all of the diagnostic categories. The predictive utility of demographic factors and pain intensity varied as a function of pain diagnosis.

CONCLUSIONS: Depressive symptoms appear to predict unemployment across a number of common chronic pain conditions. Interventions targeting depressive symptoms may thus reduce rates of unemployment in patients with a range of chronic pain diagnoses.

\section{P62}

PHANTOM LIMB PAIN IN CHILDREN AND ADOLESCENTS WITH OSTEOSARCOMA: REVEALING THE INVISIBLE

Carla Goncalves Dias, Maria Gaby de Rivero Gutierrez

Escola Paulista de Enfermagem, Universidade Federal de Sao Paulo, Sao Paulo, Brazil

AIM: To describe the patients' ability in showing phantom limb pain (PLP) and sensation through a body diagram tool, in order to improve the pain control and management.

METHODS: A case study with two oncology amputees' patients. The diagram was presented to them after surgery and at every ambulatory visit during their treatment. They were asked to draw in the institutional body diagram the experienced position and shape of the phantom limb, and the sensation and pain intensity and its characteristics.

RESULTS: The two male patients, 10 and 21 years of age, were followed up from five to seven months after amputation. The body diagram was very useful to assess the phantom limb pain and sensation, improving its control and management in both cases.

CONCLUSIONS: The case study illustrates that it is possible to provide a practical clinical tool to describe and measure phantom limb pain in pediatric patients. In addition, it allows pediatric patients and their families to participate on their own pain control improving their quality of life during the cancer treatment.

Footnotes/References: Schott GD. Revealing the invisible: The paradox of picturing a phantom limb. Brain 2013;1-10.

Burgoyne LL, Billups CA, Jirón Jr JL, et al. Phantom limb pain in young cancer-related amputees. Recent experience at st Jude Children's Research Hospital. Clin J Pain 2012;28:222-5.

\section{P63}

CHILD SELF-REPORT OF PAIN CATASTROPHIZING DOES NOT SEEM TO INFLUENCE DEGREE OF ANALGESIC USE FOR PEDIATRIC POST-OPERATIVE PAIN

Ayala Y Gorodzinsky ${ }^{1}$, Jill Chorney ${ }^{1,2}$, PORSCHE Study Group ${ }^{3}$

${ }^{1}$ Centre for Pediatric Pain Research, IWK Health Centre;

${ }^{2}$ Departments of Anesthesiology, Pain Management and

Perioperative Medicine and Psychology and Neuroscience, Dalhousie University; ${ }^{3}$ IWK Health Centre, Halifax, Nova Scotia

AIM: Pediatric post-operative pain is not well managed, especially at home where less than optimal pharmacological interventions are used (1). Individuals with higher pain catastrophizing often report high levels of fear of pain (2), so it is hypothesized that children who have higher pain catastrophizing will utilize more pain medications, as has been seen in other populations $(3,4)$

METHODS: Sixty-three 11- to 18-year-old children (89\% female) who underwent spinal fusion for adolescent idiopathic scoliosis completed the Pain Catastrophizing Scale (PCS) before surgery. Children rated their pain using a numerical rating scale and reported on number of doses of analgesics used in first week after discharge.

RESULTS: Children used a mean ( \pm SD) of $20.45 \pm 14.23$ doses of analgesics (range 0 to 59) and reported average pain ratings of $3.82 \pm 1.60$. A hierarchical regression indicated that pain ratings significantly predicted number of analgesic doses in Step 1, R2 $=0.11, \beta=0.34, \mathrm{t}(61)=2.80, \mathrm{P}<0.01$, but the addition of PCS scores in Step 2 did not significantly contribute to the model above and beyond pain scores $(\mathrm{R} 2=0.03, \beta=-0.17, \mathrm{t}(60)=-1.46, \mathrm{P}=0.15)$.

CONCLUSIONS: Children's pain scores predicted number of doses of analgesics provided; however, pain catastrophizing did not contribute to this prediction. It may be that compared to other pain populations $(3,4)$, 
there are factors associated with undergoing a planned surgery, including expected pain as a result of a scheduled surgery, that reduce the influence of pain catastrophizing on pain behaviours.

Footnotes/References:

1. Fortier MA, MacLaren JE, Martin SR, Perret-Karimi D, Kain ZN. Pediatric pain after ambulatory surgery: Where's the medication? Pediatrics 2009; 124:e588-e595.

2. Vervoort T, Goubert L, Eccleston C, Bijttebier P, Crombez G. Catastrophic thinking about pain is independently associated with pain severity, disability, and somatic complaints in school children and children with chronic pain. J Pediatr Psychol 2006;31:674-83.

3. Jacobsen PB, Butler RW. Relation of cognitive coping and catastrophizing to acute pain and analgesic use following breast cancer surgery. J Behav Med 1996;19:17-29.

4. Bédard GBV, Reid GJ, McGrath PJ, Chambers CT. Coping and selfmedication in a community sample of high school students. Pain Res Manag 1997;2:151-6.

\section{P64}

BRIDGING THE GAP BETWEEN RESEARCH AND CLINIC: A TAILORED TRAINING FOR PAIN MANAGEMENT BY ICU NURSES

Émilie Gosselin $^{1,2}$, Robin-Marie Coleman ${ }^{1,2}$, Stéphan Lavoie ${ }^{1}$, Patricia Bourgault ${ }^{1}$

${ }^{1}$ Université de Sherbrooke; ${ }^{2}$ Centre hospitalier universitaire de Sherbrooke, Sherbrooke, Quebec

AIM: Pain management in the intensive care unit is inadequate. However, specific strengths and weaknesses regarding pain management practice of intensive care unit (ICU) nurses are unknown. The aim is to evaluate the particular needs of the ICU nurses regarding pain management in order to create an adapted training for their practice.

METHODS: The particular needs of the ICU nursing staff regarding pain management were explored. To describe the practice, the Nursing Observation Tool for Pain Management (NOTPaM) was completed by three observers during 26 discrete standardized simulations. Four subscales were evaluated: Subjective assessment of pain, objective assessment of pain, intervention for pain relief and revaluation of relief and medication side effects.

RESULTS: Out of the four subscales, the objective assessment of pain and revaluation were the subscale that needed the most attention. Moreover, a large variability between the nurses' scores was noted, supporting that nursing practice is inconsistent regarding pain management. Those elements confirmed the necessity of the introduction of a behavioural pain assessment tool to facilitate nursing evaluation of non verbal patients. It also reveals the importance of a tailored training to improve evaluation.

CONCLUSIONS: Based on the results, a training was elaborated for ICU nurses. This study demonstrated that a close collaboration between researchers and clinical teachers is essential to promote enhanced nursing practice regarding pain management in the ICU. Further studies should be conducted to evaluate the impact of this association on the number and quality of pain evaluations completed by the nursing staff following the training.

\section{P65}

COMPLEMENTARY AND ALTERNATIVE MEDICINE (CAM) USE AND PERCEIVED HELPFULNESS IN A PEDIATRIC CHRONIC PAIN SAMPLE

Sophia Grewal ${ }^{1,2}$, Carlene Deits ${ }^{2}$, Tracy Anastas ${ }^{2}$, Anna C Wilson ${ }^{2}$ ${ }^{1}$ Pacific University, Forest Grove; ${ }^{2}$ Oregon Health \& Science University, Portland, Oregon, USA

AIM: This study aimed to: 1) describe rate and correlates of CAM use reported in a pediatric chronic pain sample, and 2) describe perceived helpfulness of CAM.

METHODS: Survey data was collected through a multidisciplinary outpatient pain management clinic in a university medical center children's hospital in the Pacific Northwest of the United States. The sample consisted of 240 youth with chronic pain, six to 18 years of age (mean [ \pm SD] age $4.51 \pm 2.73$ years; $65 \%$ female, $76 \%$ Caucasian), and their parents.
Children and their parents completed paper and pencil questionnaires at an initial clinic visit.

RESULTS: Most frequent primary pain locations were headache and abdominal pain, and $72 \%$ of youth reported experiencing daily pain. Most youth $(65 \%)$ had used at least one CAM modality. Fifty percent of the sample used manipulative and body based therapies (chiropractic care, massage), $42 \%$ used alternative medical systems (acupuncture, naturopathy), and 55\% used biologically-based therapies (herbal remedies, nutritional supplements). Contrary to hypotheses, CAM use was not related to child or pain characteristics, including pain intensity, duration, location, pain-related disability, child age, or child gender. Increased use of CAM treatments was related to participants' reports of increased ability to cope with their pain $(r=0.14, \mathrm{P}<0.05)$. Of those using CAM treatments $52 \%$ reported them to be helpful, while the remainder reported no benefit.

CONCLUSIONS: Providers should be aware that youth with chronic pain utilize CAM treatments, although additional research is needed to determine what predicts CAM use. Assessment of CAM use and whether patients perceive it to be helpful might inform clinical decision-making.

\section{P66 \\ THE ECONOMIC COSTS OF CHRONIC PAIN AMONG A COHORT OF TREATMENT-SEEKING ADOLESCENTS IN THE UNITED STATES}

Cornelius B Groenewald ${ }^{1}$, Bonnie Essner ${ }^{2}$, Davene Wright ${ }^{2,3}$, Megan Fesinmeyer ${ }^{2}$, Tonya Palermo 4

${ }^{1}$ Department of Anesthesiology and Pain Medicine, Seattle Children's Hospital, University of Washington School of Medicine; ${ }^{2}$ Center for Child Health, Behavior, and Development, Seattle Children's Research Institute; ${ }^{3}$ Department of Pediatrics, University of Washington School of Medicine; ${ }^{4}$ Departments of Anesthesiology and Pain Medicine, Pediatrics, and Psychiatry, Seattle Children's Hospital, University of Washington School of Medicine, Washington, USA

AIM: Adolescents with chronic pain are high consumers of health care services; however comprehensive data on the associated economic costs related to pain is limited. We therefore conducted a cost of illness study, documenting direct medical expenses and costs associated with productivity losses in families of adolescents with chronic pain.

METHODS: Participants included 149 adolescents (mean age 14.2 years; $74.5 \%$ female) and their parents recruited from multidisciplinary pain clinics across the U.S. as part of a randomized controlled trial investigating the efficacy of a web-based cognitive behavioral therapy program for chronic pain. Prior to randomization to a treatment condition, parents completed a validated measure of health and social care services, the Client Service Receipt Inventory, to report on health service use and productivity losses due to their child's chronic pain retrospectively over 12 months. Costs were calculated by multiplying reported utilization estimates by unit cost data from the 2010 Medical Expenditure Panel Survey.

RESULTS: The estimated mean and median costs per participant were $\$ 11,787$ and $\$ 6,770$ respectively. Costs were concentrated in a small group of participants; the top $5 \%$ of those patients incurring the highest costs accounted for $30 \%$ of total costs, while the lower half of participants accounted for only $15.4 \%$ of costs. Total costs to society for adolescents seeking treatment for chronic pain were extrapolated to $\$ 15.7$ billion annually in the U.S.

CONCLUSIONS: The cost of childhood chronic pain presents a substantial economic burden to families and society. Future research should focus on predictors of increased health services use and costs in children with chronic pain. 


\section{P67}

EXPLORING THE RELATIONSHIP BETWEEN SOCIAL SUPPORT, CHRONIC PAIN ACCEPTANCE, AND PSYCHOLOGICAL OUTCOMES IN INDIVIDUALS WITH ARTHRITIS

Kirsten M Gullickson, Diane L LaChapelle

University of New Brunswick, Fredericton, New Brunswick

AIM: To examine the contributions of pain acceptance and social support to depression, anxiety, and daily hassles.

METHODS: Eighty-two individuals with arthritis completed a survey measuring demographic, pain, and mental health variables. Hierarchal regressions examined the extent to which 1) demographic/pain variables (age, education level, pain severity), 2) pain acceptance (pain willingness, activity engagement), and 3) social support (punishing, distracting, solicitous) predicted depression, anxiety, and daily hassles. Significant regression results were followed up by bootstrapping mediations to examine whether acceptance mediated the relationship between social support and outcome.

RESULTS: Each group of variables contributed significantly to the prediction of depression: 1) age, education, and pain level accounted for $39.5 \%$ of the variance; 2) pain willingness and activity engagement accounted for an additional $12 \%$ of the variance; and 3) punishing responses an additional $6 \%$ of the variance. Similarly, each variable group significantly predicted anxiety: 1) education and pain levels accounted for $30.4 \%$ of the variance; 2) pain willingness and activity engagement an additional $13 \%$ of the variance; and 3) punishing responses an additional $7.5 \%$ of the variance. In the prediction of daily hassles, age and pain level accounted for $28.9 \%$ of the variance. Activity engagement and punishing responses were also significant predictors of daily hassles, but neither step accounted for a significant amount of additional variance. Activity engagement mediated the relationship between punishing social responses and each outcome. CONCLUSIONS: The mediation analyses suggest that involvement in previously enjoyed activities despite pain levels helps reduce the potential negative consequences of an unsupportive relationship.

\section{P68}

NON-STEROIDAL ANTI-INFLAMMATORY DRUG USE IN UNIVERSITY ATHLETES: KNOWLEDGE AND ATTITUDES OF RISK

Jonathon Hagel ${ }^{1}$, Dominic Hegarty ${ }^{2,3,4}$

${ }^{1}$ University College Cork School of Medicine; ${ }^{2}$ Cork University Hospital and Lee Clinic; ${ }^{3}$ University College Cork, Cork; ${ }^{4}$ Guys \& St Thomas Hospital London, London, United Kingdom

AIM: Quantify the difference in frequency of NSAID use among university athletes and non-athletes and examine differences in attitudes and behaviours associated with reported frequency of use.

METHODS: A cross-sectional study design was used whereby a validated questionnaire was distributed to all UCC medical students via email. Participation was voluntary.

RESULTS: The response rate was $24.6 \%(n=220)$. Seventy-five percent $(n=165)$ of those surveyed reported NSAID use in the past three months and of these there was no difference in the frequency of use between university athletes and non-athletes $(\mathrm{P}=0.570)$. A multinomial logistic regression in a model for the overall reason for taking NSAIDs on the frequency of use was significant $(\mathrm{P}=0.003)$. The most common reason for taking NSAIDs was pain relief. No NSAID users reported daily use.

CONCLUSIONS: There is a high prevalence of NSAID consumption in the university population and the only predictor of frequency of use was the overall reason for taking the NSAID.

\section{P69}

BEHAVIOURAL MEASURES IN A RAT MODEL OF KNEE OSTEOARTHRITIS FOR PRECLINICAL STUDY OF KNEE OSTEOARTHRITIS IN HUMAN

Clayon B Hamilton ${ }^{1}$, Micheal Pest ${ }^{2}$, Frank Beier ${ }^{3}$, Vasek Pitelka ${ }^{2}$, Anusha Ratneswaran ${ }^{2}$, Bert Chesworth ${ }^{4,5}$

${ }^{1}$ Graduate Program in Health and Rehabilitation Sciences; ${ }^{2}$ Schulich School of Medicine and Dentistry; ${ }^{3}$ Department of Physiology and Pharmacology; ${ }^{4}$ School of Physical Therapy; ${ }^{5}$ Department of Epidemiology and Biostatistics, Western University, London, Ontario

AIM: To determine pain-related behaviour and variables of exploratory behaviour in rats that distinguish between sham and a surgically-induced knee OA rat model used for preclinical study of the human condition.

METHODS: Subjects were two groups of male Sprague-Dawley rats, a sham surgery $(n=5)$ and a knee OA rat model $(n=6)$ - right knee joint destabilized by anterior cruciate ligament transection and partial medial meniscectomy as described (Appleton ART 2007). For pain-related behaviour, \% weight-bearing by the right hind limb was captured at week one, two, four, six, and eight post-surgery using an Incapacitance Tester. Exploratory behaviour (18 different variables) was assessed at week four, six, and eight post-surgery using an Open Field Tester. Micro-CT and histology were performed post-mortem at week 8. First, two-way repeated measures Analysis of Variance tested for significant difference (two-tailed $\mathrm{P}<0.05)$ in pain-related behaviour and each exploratory behaviour between the study groups and overtime. Second, eight exploratory behaviour variables were selected and tested as predictors of study group status using logistic regression.

RESULTS: Micro-CT confirmed knee OA. Weight-bearing was significant as a main effect $(F 1,9)]=24.591 ; P=0.001)$, and at week two, six, and eight postsurgery. Exploratory behaviour variables showed no main effect by group, nine variables had a main effect by time, and three variables had an interaction effect. Logistic regression revealed weight-bearing and only the same three exploratory variables (ambulatory episode, vertical episode, and counterclockwise revolution) were significant predictors of study group status.

CONCLUSIONS: These variables may be useful measures in preclinical pharmacological studies using this knee OA model.

Footnotes/References: Appleton CT, McErlain DD, Pitelka V, et al. Forced mobilization accelerates pathogenesis: Characterization of a preclinical surgical model of osteoarthritis. Arthritis Res Ther 2007;9:R13. doi: $10.1186 /$ ar2120.

\section{P70}

\section{KNOWLEDGE TRANSLATION AND CHRONIC PAIN IN OLDER ADULTS: A SYSTEMATIC REVIEW}

Amy DI Hampton, Michelle M Gagnon, Thomas Hadjistavropoulos University of Regina, Regina, Saskatchewan

AIM: Given the aging population and the high prevalence of chronic pain in older persons, the under-management of pain is a significant health and economic concern. The inadequate implementation of many pain assessment/management procedures suggests that there may be a failure of translating knowledge into practice. Our objective was to conduct a systematic literature review of knowledge translation $(\mathrm{KT})$ programs pertinent to chronic pain in older persons with the aim of identifying effective KT initiatives.

METHODS: Searches of MEDLINE, CINAHL, Web of Science ${ }^{\circledR}$, and Cochrane Library were conducted using key search terms. Original articles written in English with a primary focus on evaluating pain assessment/ management KT initiatives pertinent to older persons were included.

RESULTS: A total of $16 \mathrm{KT}$ initiatives targeting health care providers (HCP) and $27 \mathrm{KT}$ initiatives targeting patients were identified. Education for HCP resulted in gains in knowledge as well as increased staff-reported use of pain assessment tools and management strategies. Although there has been an insufficient focus on patient outcomes resulting from HCP KT initiatives, the few studies that examined patient outcomes demonstrated reductions in the prevalence and severity of pain. Although KT initiatives targeting patients have not consistently demonstrated patient gains, there 
have been improvements in pain levels, mood, and use of coping strategies.

CONCLUSIONS: KT initiatives have resulted in positive gains when directed at both HCP and older adults with chronic pain. Given the undermanagement of pain in older adults, there is a pressing need to increase the systematic implementation of large-scale KT initiatives.

\section{P71}

\section{PSYCHOLOGICAL DETERMINANTS OF EXPECTANCIES FOR RETURN TO WORK AND RECOVERY IN WHIPLASH- ASSOCIATED DISORDERS}

Johanna Harrison ${ }^{1}$, Zina Trost ${ }^{2}$, Michael Sullivan ${ }^{1}$

${ }^{1}$ McGill University, Montreal, Quebec; ${ }^{2}$ University of North Texas, Denton, Texas, USA

AIM: Psychosocial factors, including expectancies, have emerged as prognostic factors for a variety of musculoskeletal disorders, including WAD. Our current understanding of the role that patient expectancies play in recovery from musculoskeletal pain conditions, including WAD, is preliminary and expanding. However the determinants of expectancies remain unclear, as does the impact of the determinants on the changes in patient expectancies. The aim of the present study was to prospectively examine patients' specific expectancies for recovery during a rehabilitation intervention for WAD in order to investigate the determinants of expectancies, and the influence of psychological factors on the change in expectancy measures. We were particularly interested in understanding how the changes in various psychological factors over the course of the rehabilitation program would influence the change in expectancies.

METHODS: The study sample consisted of 153 individuals who had sustained whiplash injuries (grade 1 or 2) in rear-collision motor vehicle accidents. Participants were enrolled in a standardized 7-week multidisciplinary functional restoration rehabilitation programs aimed at fostering recovery from whiplash injury. Measures of pain severity, Self-rated disability, pain catastrophizing, pain-related fear of movement, and expectancies for recovery were completed at initial assessment, mid-way through the program, and during the last week of the program.

RESULTS: Analyses revealed that the expectancy for decreased pain at admission was significantly correlated to patients' ratings of return to work and resumption of social-recreational activities at the end of treatment. Univariate analysis revealed that patient expectancies for decreased pain, improved sleep, and resumption of social-recreational activities were significantly negatively correlated with patients' levels of pain catastrophizing (PCS), and pain-related fear of movement (TSK), but not with expectancies for RTW. Further multivariate analyses revealed only PCS account for unique variance in the expectancy for decreased pain, improved sleep, and return to social and recreational activities. Additional analyses of the changes in psychological factors over the course of the rehabilitation program revealed that they were predictive of the changes in the patients' expectancies over the same period.

CONCLUSIONS: The findings of this study provide some evidence regarding the psychological determinants of patient expectancies for recovery, including RTW, and provide potential targets for interventions to modify patients' expectancies.

\section{P72}

\section{ATTENTION BIAS TO FACIAL EXPRESSIONS OF PAIN IN ADOLESCENTS: EXAMINING EFFECTS OF PAIN-RELATED ANXIETY AT EARLY AND LATE STAGES OF ATTENTION PROCESSING}

Lauren C Heathcote ${ }^{1}$, Elaine Fox ${ }^{1}$, Christopher Eccleston ${ }^{2}$, Jennifer YF Lau ${ }^{3}$

${ }^{1}$ University of Oxford, Oxford; ${ }^{2}$ University of Bath, Bath; ${ }^{3}$ Institute of Psychiatry, Kings College London, London, United Kingdom

AIM: A significant number of children and adolescents report distressing and disabling pain. Moreover, onset of chronic pain disorders early in life may be predictive of chronicity. In adult populations, attention biases toward pain-related stimuli are thought to contribute to pain and pain behaviours in both acute and chronic pain conditions. However, the precise nature of the association between attention biases and pain experiences is poorly understood, especially in paediatric samples, for which there are only a handful of studies to date. Therefore, the present study investigated the relationship between pain-related anxiety and selective attention to pain in a sample of adolescents.

METHODS: Eighty pain-free adolescents (16 to 18 years of age) performed a dot-probe task with pictorial stimuli displaying pain or neutral facial expressions, presented for $100 \mathrm{~ms}$ and $1250 \mathrm{~ms}$, thought to reflect early and later stages of processing respectively. Participants also completed fear of pain, general anxiety, and attention control measures.

RESULTS: There was a significant difference in attention bias between adolescents with low and high fear of pain. Adolescents with low fear of pain were significantly slower on congruent trials at $100 \mathrm{~ms}$, indicating avoidance of pain faces, however this bias was absent in adolescents with high fear of pain. Moreover, attention control was significantly associated with fear of pain and attention bias at $1250 \mathrm{~ms}$.

CONCLUSIONS: The present data speak to the linkage between attention biases and pain experiences. Targeting attention biases could be a useful therapeutic tool for chronic pain disorders, for example through Attention Bias Modification training.

\section{P73}

NEW DEDICATED COURSE ON PAIN IN A MASTER'S LEVEL PHYSIOTHERAPY PROGRAM

Kadija Perreault ${ }^{1}$, Hélène Moffet ${ }^{1,2}$, Nathalie Mathieu ${ }^{1,3}$, Jean-François Roux ${ }^{1,3}$, Isabelle Savard ${ }^{1}$, Richard Debigaré ${ }^{1,4}$ ${ }^{1}$ Faculty of Medicine, Université Laval; ${ }^{2}$ Centre interdisciplinaire de recherche en réadaptation et intégration sociale (CIRRIS), Institut de réadaptation en déficience physique de Québec, Quebec; ${ }^{3}$ Centre Hospitalier Universitaire Affilié (CHAU) Hôtel-Dieu de Lévis, Alphonse-Desjardins Health and Social Services Centre, Lévis; ${ }^{4}$ Research Center of the Quebec Heart and Lung Institute, Laval, Quebec

AIM: Research in the last decades has put forward the lack of time devoted to pain in healthcare providers' basic training, including physiotherapists, to whom people experiencing pain often turn for relief. At Université Laval, a new course dedicated to pain has been integrated into the master's physiotherapy program. This presentation describes its development, implementation and evaluation.

METHODS: The $30 \mathrm{~h}$ mandatory course aims to enable physiotherapy students to plan strategies for pain prevention and management for people of all ages, considering its multidimensional nature. The course includes a mix of lectures and workshops given by practicing clinicians and was developed based on IASP curricula and program needs. The course was evaluated using student surveys, discussions with educators and students' results on an endof-training Objective Structured Clinical Examination (OSCE) station.

RESULTS: In the two years the course has been given, students appreciated the course $(98 \% ; n=80)$ and reported on the value of acquiring painrelated knowledge, notably psychosocial issues and how to address them in practice. Challenges identified by students and educators included covering this complex subject within time-constraints and integrating practical learning activities. Students' results on the OSCE station demonstrated that most students were successful in demonstrating the expected skills and behaviours (mean performance $72 \pm 12 \%$ ) in a simulation environment. CONCLUSIONS: The integration of a dedicated course on pain in a master's physiotherapy program fills a gap in the training of future physiotherapists. Work is underway to continue improving this training, assess its impact, and adapt it to emerging evidence in the field. 


\section{P74}

INTERPROFESSIONAL INTERACTIONS FOR LOW BACK PAIN MANAGEMENT: PERCEPTIONS OF PRIVATE SECTOR PHYSIOTHERAPISTS

Kadija Perreaultt, ${ }^{1,2}$, Clermont E Dionne ${ }^{1,2,3}$, Michel Rossignol ${ }^{4,5}$, Stéphane Poitras ${ }^{6}$, Diane Morin ${ }^{2,7}$

${ }^{1}$ Centre for Interdisciplinary Research in Rehabilitation and Social Integration (CIRRIS), Institut de réadaptation en déficience physique de Québec; ${ }^{2}$ Université Laval; ${ }^{3}$ Centre de recherche FRQS du Centre hospitalier universitaire (CHU) de Québec; ${ }^{4}$ Institut national d'excellence en santé et en services sociaux; ${ }^{5}$ Department of Epidemiology, Biostatistics and Occupational Health, McGill University; ${ }^{6}$ School of Rehabilitation Science, Faculty of Health Sciences, University of Ottawa; ${ }^{7}$ Institut universitaire de formation et de recherche en soins, Université de Lausanne, Lausanne, Switzerland

AIM: Research on interventions for low back pain (LBP) supports the involvement of multiple healthcare providers. Physiotherapists often contribute to the management of LBP, especially in the private sector, but little is known on their interprofessional interactions. The aim of this study was to identify private sector physiotherapists' perceptions regarding the need for interprofessional interactions for LBP management.

METHODS: Data were collected through a mixed-methods study, combining semi-structured interviews $(n=13)$ followed by a province-wide online survey of private sector physiotherapists in Québec ( $n=327$; participation $67.7 \%$ ), and analysed using qualitative content analysis and descriptive statistics.

RESULTS: Interviewed physiotherapists explained they needed interprofessional interactions depending on stage of the condition (persistent rather than acute), course of recovery, presence of adverse psychosocial factors, and financial compensation issues. For some of the physiotherapists, LBP was a standout condition for which interprofessional interactions are very important. A majority of surveyed physiotherapists (54.4\%) also agreed that they needed to interact with other providers to adequately intervene with individuals with LBP, while $30.3 \%$ felt that such interactions were more important for these individuals than for those consulting for other conditions.

CONCLUSIONS: Physiotherapists' perceived need for interprofessional interactions for LBP management is influenced by different aspects of the individuals' conditions. These results are important for the development of strategies aiming to increase interprofessionalism in the management of LBP. Further studies are warranted to specify the types of interactions to promote, their timing, frequency and impact on patient and provider outcomes.

\section{POSTERS THURSDAY MAY 22, 2014}

\section{P80}

CULTURAL ADAPTATION OF OBSERVATIONAL PAIN ASSESSMENT MEASURES: A METHODOLOGICAL EXAMPLE USING THE COMFORT BEHAVIORAL SCALE

Randi Dovland Andersen ${ }^{1,2,3}$, Leena Jylli ${ }^{4}$, Bruce Ambuel ${ }^{5}$ ${ }^{1}$ Department of Child and Adolescent Health Services, Telemark Hospital, Skien, Norway; ${ }^{2}$ Department of Neurobiology, Care Sciences and Society, Karolinska Institutet, Stockholm, Sweden; ${ }^{3} \mathrm{CIHR}$ Strategic Training Program on Pain in Child Health (PICH); ${ }^{4}$ Department of Clinical Science, Intervention and Technology, Karolinska Institutet, Stockholm, Sweden; ${ }^{5}$ Department of Family \& Community Medicine, Medical College of Wisconsin, Wisconsin, USA

AIM: There is little empirical evidence regarding the translation and cultural adaptation of observational pain assessment measures. Studies that evaluate and further develop existing practices are needed. This study explores the use of cognitive interviews in the translation and cultural adaptation process, using the COMFORT behavioral scale as an example, and demonstrates a structured approach to the analysis of data from these interviews.
METHODS: Qualitative study; individual cognitive interviews based on assessment with the COMFORT behavioral scale. $n=12$; eight nurses, three physicians and one nurse assistant. The clinician first read and then used the COMFORT behavioral scale to assess pain based on information gained from a film vignette. Two interview techniques were applied; Thinking Aloud, where the participant verbalized his/her thought process, and Verbal Probing where the interviewer asked specific questions related to understanding of the measure, information recall and the decision process. A structured, iterative method to analyze interviews and tabulate results was applied.

RESULTS: Our analysis revealed two categories of problems: 1) Scale problems, warranting a change in the wording of the scale, including a) translation errors, b) content not understood as intended, and c) differences between the original COMFORT scale and the revised COMFORT behavioral scale; and, 2) Rater-context problems caused by a) unfamiliarity with the scale, b) lack of knowledge and experience, and c) assessments based on a film vignette.

CONCLUSIONS: Identified scale problems were used to refine the translated measure. Cognitive interviews seem a valuable complement to existing translation and cultural adaptation guidelines.

\section{P81}

EXAMINING THE RELATIONSHIP BETWEEN CHRONIC PAIN AND FUNCTIONING ACROSS TIME - DO IMPROVEMENTS IN FUNCTIONAL DISABILITY PRECEDE REDUCTIONS IN PAIN?

Lenny R Evans ${ }^{1,2}$, Keri R Hainsworth ${ }^{1,2}$, Yumei Cao ${ }^{1}$, Kim Anderson Khan ${ }^{1,2}$, Renee Ladwig', Kristen E Jastrowski Mano ${ }^{3}$, W Hobart Davies ${ }^{4}$, Steven J Weisman ${ }^{1,2}$

${ }^{1}$ Medical College of Wisconsin; ${ }^{2}$ Childrens Hospital Wisconsin, Milwaukee, Wisconsin; ${ }^{3}$ Alliant International University, California School of Professional Psychology, Alhambra, California;

${ }^{4}$ University of Wisconsin - Milwaukee, Milwaukee, Wisconsin, USA AIM: Clinical wisdom dictates that functioning returns before chronic pain improves. Although this principle is a cornerstone of pain treatment, few empirical investigations have been published on this important question. The purpose of this study was to examine the relationship of pain and functioning across time, specifically testing whether changes in functioning precede future reductions in pain among youth aged eight to 18 presenting for treatment in an outpatient chronic pain clinic.

METHODS: Structural equation modeling via M-Plus was utilized to examine the relationships between worst pain intensity, as measured by the NRS-11, and functional disability, as measured by the Child Activity Limitations Questionnaire, at intake and one-, three- and 12-month follow-up.

RESULTS: Direct effects suggested early improvements in functional disability preceded small but statistically and clinically meaningful decreases in pain at three months. Decreases in pain at three months preceded large improvements in functional disability at one-year follow-up. Indirect effects suggested pain was not associated with changes in functioning while improvements in early functioning (intake, one-month follow-up) preceded later reductions in pain (three-, 12-month follow up).

CONCLUSIONS: This study provides preliminary support for the premise that functioning begins to improve before pain. It also suggests that mid-treatment reductions in pain precede subsequent decreases in functional disability. These findings lend support to a hallmark principle of pediatric chronic pain treatment - focusing on reducing functional disability first - with the caveat that although early gains in functioning may be more clearly evident than clinically meaningful decreases in pain, pain reductions precede important late-treatment gains in functioning. 
P82

EVALUATING PAIN BEHAVIORS IN THE ADULT INTENSIVE CARE UNIT WITH VIDEO RECORDING: A RELIABLE RESEARCH METHOD

Céline Gélinas ${ }^{1}$, Jane Topolovec-Vranic ${ }^{2,3}$, Caroline Arbour ${ }^{4}$, Melody Ross $^{1}$, Daniel Shripts ${ }^{5}$, Laureanne Khouri ${ }^{6}$, Darina Tsoller ${ }^{6}$ ${ }^{1}$ McGill University, Montreal, Quebec; ${ }^{2}$ St Michael's Hospital; ${ }^{3}$ University of Toronto, Toronto, Ontario; ${ }^{4}$ Hôpital du Sacré-Coeur de Montréal, Montreal, Quebec; ${ }^{5}$ York University, Toronto, Ontario; ${ }^{6}$ Jewish General Hospital, Montreal, Quebec

AIM: Although video recording is common in pain research, this method has only more recently been applied to the adult intensive care unit (ICU). This study aimed to examine interrater and intrarater reliability of coded pain behaviors of video-recorded ICU patients with a traumatic brain injury (TBI) by trained raters.

METHODS: Four raters underwent a $3 \mathrm{~h}$ training session and practiced video coding of pain behaviors using a comprehensive checklist derived from two existing pain assessment tools (ie, Critical-Care Pain Observation Tool, and Pain Behavioral Assessment Tool). Then, they independently coded pain behaviors of 20 critically ill TBI patients who were video recorded during two types of procedures: a) non-nociceptive: non-invasive blood pressure measurement (NIBP), and b) nociceptive: turning. Two raters recoded the videos one month later for intrarater reliability.

RESULTS: Most of the 20 ICU patients were males ( $n=13$ [65\%]), with an average age of 52 years (range 18 to 84 years of age), and suffered from moderate to severe TBI (85\%). Intraclass correlation coefficients (ICC) of coded total pain behaviors between the four raters were 0.86 and 0.91 during NIBP and turning, respectively. The lowest ICC (0.52) was found for muscle tension during NIBP. In terms of intrarater reliability, ICC varied from 0.65 to 0.93 for each rater.

CONCLUSIONS: Although video recording appears to be a reliable method for the evaluation of pain behaviors, some of them may be more difficult to observe consistently on video. Supplementing video-recorded data with real-time observations may help to capture more robust data.

\section{P83}

TOO MANY SCREAMING BABIES: A SYSTEMATIC REVIEW OF PAIN MANAGEMENT PRACTICES DURING IMMUNIZATIONS ON YOUTUBE

Denise Harrison $^{1,2,3}$, Margaret Sampson ${ }^{4}$, Jessica Reszel ${ }^{1}$, Jordi Cumber ${ }^{1}$, Catherine Pound ${ }^{4}$, Ann Fuller ${ }^{4}$, Kowsar Abdulla ${ }^{1}$, Nick Barrowman ${ }^{1}$, Stuart Nicholls ${ }^{5}$

${ }^{1}$ Children's Hospital of Eastern Ontario Research Institute; ${ }^{2}$ School of Nursing, University of Ottawa; ${ }^{3}$ Clinical Epidemiology Program, Ottawa Hospital Research Institute; ${ }^{4}$ Children's Hospital of Eastern Ontario; ${ }^{5}$ Department of Epidemiology and Community Medicine, University of Ottawa, Ottawa, Ontario

AIM: Despite the abundance of effective procedural pain management strategies for infants, these strategies are not consistently used during immunizations. The aim of this study was to conduct a systematic review of YouTube videos showing intramuscular injections in infants to evaluate use of pain management strategies and infants' pain responses.

METHODS: A systematic review of YouTube was completed between July 2012 and January 2013. Using set inclusion and exclusion criteria and the discontinuation rule, 142 videos were included. Each video was coded by two viewers for variables including age of infant, number of injections, crying incidence and duration, observable pain management, and pain score using the FLACC (Face, Legs, Activity, Cry, Consolability) tool.

RESULTS: Most infants received one injection (range of one to six). 95\% of infants cried during and following injections, and the average crying time was $40 \mathrm{~s}$. No videos showed breastfeeding or use of sucrose/any sweet solutions during the procedure. Observable positioning and distraction techniques during injections were evident in $38 \%$ and $11 \%$ of videos, respectively. FLACC scores at time of injection were high, with a median of $10(\mathrm{IQR}=3)$.

CONCLUSIONS: This systematic review of YouTube videos of infant immunizations highlighted that infants were highly distressed yet use of recommended pain management strategies were rare. Results will be used as a baseline to evaluate the impact of future knowledge translation interventions to improve pain management practices via the medium of YouTube.

\section{P84}

TOO MUCH EVIDENCE? SWEET SOLUTIONS FOR NEWBORN PAIN MANAGEMENT

Denise Harrison $^{1,2,3}$, Mariana Bueno ${ }^{4}$, Jessica Reszel ${ }^{1}$, Yehudis Stokes $^{1}$, Lucy Turner ${ }^{5}$

${ }^{1}$ Children's Hospital of Eastern Ontario Research Institute; ${ }^{2}$ School of Nursing, University of Ottawa; ${ }^{3}$ Clinical Epidemiology Program, Ottawa Hospital Research Institute, Ottawa, Ontario; ${ }^{4}$ Department of Maternal-Child and Public Health, School of Nursing, Federal University of Minas Gerais, Belo Horizonte, Brazil; ${ }^{5}$ Knowledge Synthesis Group, Ottawa Hospital Research Institute, Ottawa, Ontario

AIM: To systematically review published studies evaluating sweet solutions for procedural pain reduction in infants to ascertain when there was sufficient evidence of analgesic effectiveness.

METHODS: Databases searched included Medline, Embase, Cumulative Index to Nursing and Allied Health Literature, and PsycINFO. Cumulative meta-analyses using outcome measures of crying duration (s) and standardized mean differences (SMD) in composite pain scores were conducted. RESULTS: 200 studies were identified, of which 179 (90\%) had placebo/ no-treatment groups. Cumulative meta-analysis for crying time included 22 trials and showed that from the first included RCT in 1995, the mean reduction in cry time for sweet solutions compared to placebo was $-14.14 \mathrm{~s}$ ( $95 \%$ CI -17.23 s to $-11.05 \mathrm{~s}$ ). Cumulative meta-analysis of the 22 RCTs shows a 22.40 s reduction ( $95 \%$ CI -37.98 s to -18.82 s). Cumulative meta-analysis for SMD for pain scores included 45 trials and showed that from the second included RCT in 1999, the SMD in pain scores for sweet solutions compared to placebo during painful procedures was -1.01 ( $95 \%$ CI -1.94 to -0.07 ) Cumulative meta-analysis of the 45 RCTs conducted up to 2013 shows a -0.99 point-reduction in SMD pain scores (95\% CI -1.21 to -0.76 ).

CONCLUSIONS: A state of equipoise relating to sweet-taste analgesia during single painful procedures in newborns has not existed since 1995 for crying time, and 1999 for pain scores. It is unethical to conduct RCTs of sweet solutions with a placebo/non-treatment group. Sucrose or glucose should be considered standard of care for neonatal procedural pain trials.

\section{P85}

SAFETY PROFILE OF DOXEPIN 3 MG AND 6 MG IN ELDERLY INSOMNIA PATIENTS: AN EXAMINATION OF THE DATA THAT INFLUENCED RECENT UPDATES TO THE BEERS CRITERIA

\section{H Heith Durrence}

\section{San Diego State University, San Diego, California, USA}

AIM: Pain and insomnia are two common health-related complaints that are often comorbid and that increase in prevalence with age. The American Geriatrics Society updated the Beers Criteria for potentially inappropriate medication use in older adults in 2012. These changes clarified that doxepin (DXP) doses $\leq 6 \mathrm{mg}$ are considered appropriate for use in the elderly population. This report reviews data of DXP (Silenor) $3 \mathrm{mg}$ and $6 \mathrm{mg}$ in elderly insomnia patients that were the basis for these changes. METHODS: Safety endpoints from two double-blind placebo-controlled trials of elderly insomnia patients are reported. Study A was a 12-week trial and Study B was a four-week trial. Safety endpoints assessed include adverse event (AE) reporting, next-day residual effects, and sleep architecture.

RESULTS: Overall, DXP 3 mg and 6 mg were well-tolerated in both studies, with no apparent dose-related effects compared with placebo (PBO). Rates of treatment-emergent AEs for DXP $3 \mathrm{mg}$ and $6 \mathrm{mg}$ were similar to $\mathrm{PBO}$ in both studies. In terms of next-day residual effects, there were no significant differences between PBO and DXP $3 \mathrm{mg}$ at any time point in Study A. There were no reports of complex sleep behaviors, memory impairment or cognitive disorder in any DXP-treated patient. 
CONCLUSIONS: Data indicate that DXP $3 \mathrm{mg}$ and $6 \mathrm{mg}$ were highly efficacious and well-tolerated, with no evidence of REM suppression or anticholinergic effects. Most relevant for this patient population, DXP at these doses significantly improved endpoints associated with sleep maintenance and early morning awakenings without evidence of next-day residual effects.

\section{P86}

\section{WITHDRAWN}

\section{P87}

\section{DOES AGING AFFECT THE RELATIONSHIP BETWEEN LOW BACK PAIN AND DISABILITY?}

Francis Houde $^{1}$, François Cabana ${ }^{1,2}$, Guillaume Léonard ${ }^{1}$ ${ }^{1}$ Centre de recherche sur le vieillissement du CSSS-IUGS; ${ }^{2}$ Centre hospitalier universitaire de Sherbrooke, Sherbrooke, Quebec

AIM: Previous studies have revealed a moderate association between pain intensity and physical disability in patient with low back pain. To our knowledge, however, no studies have evaluated if this association is different between young and old individuals. The aim of the present study was to evaluate the relationship between pain intensity and physical disability in a population of young and old patients presenting low back pain.

METHODS: Pain intensity (visual analog scale) and physical disability (Oswestry Disability Index) taken from the medical record of 164 chronic low back pain patients were analyzed. The sample was divided into two groups, using a median split approach (young group: 22 to 48 years of age, $n=82$; old group: 49 to 90 years of age, $n=82$ ). For each group, correlational analyses were used to determine the association between pain intensity and physical disability. A Fisher r-to-z transformation was performed to test for between-group difference.

RESULTS: There was a significant and positive association between pain intensity and physical disability for both young and old individuals. However, the correlation was stronger in the younger group $(r=0.66 ; \mathrm{P}<0.01)$ compared to the older group $(r=0.44 ; \mathrm{P}<0.01)$ (Fisher $\mathrm{Z}=2.03 ; \mathrm{P}<0.05)$. CONCLUSIONS: Although both young and old individuals showed a significant association between pain intensity and physical disability, current results indicate that the relationship between these two factors is more tenuous in older individuals than in young individuals. Future research is essential to identify the factors underlying this age-related difference.

\section{P88}

PREVALENCE AND DETERMINANTS OF CANNABINOID PRESCRIPTION FOR THE MANAGEMENT OF CHRONIC NON-CANCER PAIN: A POSTAL SURVEY OF PHYSICIANS Huguette St-Amant ${ }^{1}$, Mark Ware ${ }^{2}$, Nancy Julien ${ }^{1}$, Anaïs Lacasse ${ }^{1}$ ${ }^{1}$ Département des sciences de la santé, Université du Québec en Abitibi-Témiscamingue (UQAT), Rouyn-Noranda; ${ }^{2}$ The Alan Edward Pain Management Unit, McGill University Health Centre, Montreal, Quebec

AIM: Measure prevalence and determinants of cannabinoid prescription for the management of chronic non-cancer pain (CNCP).

METHODS: : In February 2013, a postal survey was sent to all physicians of the region of Abitibi-Témiscamingue, Quebec, using a modified Dillman method. Measures included the past-year prevalence of cannabinoid prescription for the management of CNCP, characteristics of physicians' medical practice, and factors that could increase physicians' comfort level with cannabinoid prescribing. Univariate and multivariate logistic regression models were used to identify determinants of cannabinoids prescription. RESULTS: The response rate was $52.2 \%$, for a total of 166 physicians (family physicians: $56 \%$ ). A majority of physicians (79.2\%) did not attend continuing medical education activities about cannabinoids in the past year. The prevalence of cannabinoid prescription for the management of CNCP was $23.0 \%(91.1 \%$ of these physicians prescribed cannabinoids to $\leq 5$ patients). Among prescribers, $92.1 \%$ reported having prescribed nabilone, $18.4 \%$ medical marijuana, $5.3 \% \mathrm{THC} /$ cannabidiol, and $0 \%$ dronabinol. Adjusting for physicians' years of practice, speciality, practice setting, caseload, and perceived $\mathrm{CNCP}$ prevalence, multivariate models showed that physicians' comfort level with cannabinoid prescribing for the management of CNCP (OR 1.25 [95\% CI 1.01 to 1.55]) was the only determinant of an increased likelihood of prescription. Prescribers and non-prescribers reported that most important factors that could increase their comfort level with cannabinoid prescribing for the management of $\mathrm{CNCP}$ were continuing medical education, guidelines/algorithms including cannabinoids, and clinical data/ studies (reported by $\geq 50 \%$ of physicians).

CONCLUSIONS: : Results suggest a low prevalence of cannabinoid prescribing for the management of $\mathrm{CNCP}$ and this study will help the planification of continuing medical education.

\section{P89}

\section{CHARACTERISTICS AND IMPACT OF CHRONIC PAIN IN ADULTS WITH CYSTIC FIBROSIS}

Ange C lliza ${ }^{1}$, Yves Berthiaume ${ }^{2}$, Alphonse Jeanneret ${ }^{2}$, Anne-Marie Lavoie-Pilote ${ }^{2}$, Manon Choinière ${ }^{1,3}$

${ }^{1}$ Centre de recherche du Centre hospitalier de l'Université de Montréal (CHUM); ${ }^{2}$ Clinique de fibrose kystique du CHUM; ${ }^{3}$ Groupe de recherche sur la douleur chronique, Montreal, Quebec AIM: Cystic fibrosis (CF) is an inherited chronic disease which affects respiratory and gastrointestinal systems. CF management has greatly improved in recent decades, thereby increasing life expectancy and improving quality of life (QOL) of affected people. However, few studies have addressed the problem of persistent pain in CF patients and its impact on their QOL. The aims of this study were 1) to assess the prevalence and characteristics of the pain associated with CF, 2) to evaluate and compare health-related QOL and emotional well-being in CF patients with and without pain problems, and 3) to

METHODS: A descriptive cross-sectional study was conducted among adult patients followed at the CF Clinic of the CHUM. This study used a set of validated self-administered questionnaires: Brief Pain Inventory, Cystic Fibrosis Questionnaire-Revised (CFQ-R) to measure QOL, and Hospital Anxiety and Depression Scale. Patients' medical charts were also reviewed to assess the quality of analgesic practices.

RESULTS: Of the 295 patients enrolled in this study, 205 patients $(69.5 \%)$ completed the questionnaires. Average age was $31.9 \pm 9.2$ years, and $51.2 \%$ were females. Sixty percent $(n=123)$ of the participants reported having experienced CF-related pain in the past 12 months. Close to $20.0 \%$ of them had pain continuously. Pain intensity in the past seven days was rated $\geq 4 / 10$ by $44.7 \%$ of patients. The impact of pain on various aspects of daily living was moderate or severe in $29.3 \%$ of the patients. The mean scores on all CFQ-R QOL subscales (except for weight disturbance) were significantly lower in patients with pain compared to those without pain $(\mathrm{P}<0.001)$. A significantly greater $\%$ of patients with pain also exhibited signs of anxiety $(\mathrm{P}=0.001)$ and depression $(\mathrm{P}=0.003)$.

CONCLUSIONS: Persistent pain is prevalent in adult CF patients, which pain can have a significant impact on their daily activities, QOL, and emotional well-being. This type of CF-related complication is nonnegligible, and should be properly assessed and managed to provide $\mathrm{CF}$ patients with optimal care.

\section{P90}

\section{"THERE'S A PAIN APP FOR THAT": REVIEW OF PATIENT- TARGETED SMARTPHONE APPLICATIONS FOR PAIN MANAGEMENT}

$\underline{\text { Lindsay Jibb }}^{1}$, Chitra Lalloo ${ }^{2}$, Arnav Agarwal ${ }^{2}$, Jennifer Stinson ${ }^{1}$ ${ }^{1}$ Hospital for Sick Children, Toronto; ${ }^{2}$ McMaster University, Hamilton, Ontario

AIM: To characterize currently available smartphone apps for people with pain and critically appraise app content and functionality with respect to the ability of the app to support pain self-management.

METHODS: An electronic search was conducted between March and May 2013 of the official app stores for the four major smartphone operating systems. Two authors independently identified patient-focused apps with a stated goal of pain treatment. Discrepancies regarding selection were resolved through discussion with a third party. Metadata from all included apps were abstracted into a standard form. The content and functionality of each app as it pertained to pain self-management was rated. 
RESULTS: A total of 221 apps met the inclusion criteria. Self-care skill support was the self-management function most frequently incorporated in the identified apps (81\%). Apps also purported providing patients with the ability to engage in pain education (61\%), self-monitoring (14\%), social support (3\%) and goal-setting (1\%). No apps were comprehensive in terms of pain self-management functionality, with the majority of apps including only a single self-management function. Additionally, $<10 \%$ of apps including any healthcare professional in their development, no apps reported a theoretical rationale for features or design and only one app had been the subject a scientific effectiveness testing.

CONCLUSIONS: Currently available pain self-management apps are simplistic, lack the involvement of healthcare professionals in their development and have not been rigorously tested for effectiveness on painrelated health outcomes. There is a need to develop and test theoretically- and evidence-based apps to better support patients with remote and real-time pain self-management.

\section{P91}

\section{SUCCESSFULLY NAVIGATING THE STRESS AND PAIN OF SURGERY: PARENTS' EXPERIENCES OF CARING FOR THEIR CHILDREN IN THE PERIOPERATIVE PERIOD}

Kailyn Jones ${ }^{1,2}$, Jill Chorney ${ }^{2,3}$, Karen Archibald ${ }^{2}$, Paula Forgeron ${ }^{4}$ ${ }^{1}$ Mount Saint Vincent University; ${ }^{2}$ IWK Health Centre; ${ }^{3}$ Dalhousie University, Halifax, Nova Scotia; ${ }^{4}$ University of Ottawa, Ottawa, Ontario

AIM: The objective of the current study was to investigate parents' perceptions of their preparation for their child's day surgery.

METHODS: This descriptive qualitative study used semi-structured interviews to capture perceptions of parent preparedness to care for their child before, during and after day surgery at the IWK Health Centre in Halifax, Nova Scotia. Semantic thematic analysis was used to analyze participant experiences.

RESULTS: Three themes emerged. Caring for and Supporting Personal Needs describes the ways in which parents address their own personal needs caused by stress and worry about their child's surgery. Caring for and Supporting Childs Needs describes the ways in which parents manage and address the needs of their child. Pain care was a major focus within this theme. While parents discussed engaging in pain management strategies, they voiced feeling underprepared to manage their child's pain and felt uncertain about how to prepare their child for pain following surgery. Building Trusting Relationships describes the critical importance parents identified in establishing rapport with the healthcare staff they encounter. CONCLUSIONS: The current study examined supports parents received and areas in which parents felt support was lacking throughout their preparation experience. Although they received information on perioperative care they faced challenges in caring for themselves and their child. Pain care remains a primary concern. Clinicians need to take a holistic approach in preparing parents to care for their child throughout their surgical experience to ensure they are adequately equipped to care for themselves and their child.

\section{P92}

PAIN IN EXTREMELY LOW BIRTH WEIGHT SURVIVORS: SEVERITY AND STABILITY FROM ADOLESCENCE TO ADULTHOOD

Jordana Waxman ${ }^{1}$, Ryan J Van Lieshout ${ }^{2}$, Saroj Saigal ${ }^{2}$, Michael H Boyle ${ }^{2}$, Louis A Schmidt ${ }^{2}$

${ }^{1}$ York University, Toronto; ${ }^{2}$ McMaster University, Hamilton, Ontario AIM: The objective of this study was to compare the level and stability of pain concerns in those born at extremely low birth weight (ELBW; $<1000 \mathrm{~g}$ ) and normal birth weight (NBW) from 12 to 16 years of age, to 30 to 35 years of age.

METHODS: Longitudinal methods were employed to follow 84 ELBW survivors born between 1977 and 1982 and 78 socio-demographically matched NBW controls. Participants were asked to rate pain concerns at 12 to 16 (teen), 22 to 26 (young Adult), and 30 to 35 (adult) years of age. A composite measure of physical pain was derived at each visit by summing five variables on the somatic scale of the Child Behaviour Checklist/Young Adult Self-Report. Items included "aches and pains," "headaches," "nausea," "skin problems," and "stomach aches."

RESULTS: When using an ANOVA with repeated measures with a Greenhouse-Geisser correction, the mean scores for physical pain were statistically different across time, $\mathrm{F}(1.959,313.460)=3.967, \mathrm{P}=0.02$, but not between groups.

CONCLUSIONS: Individuals born at ELBW did not differ on a composite measure of physical pain concerns than their NBW controls. Overall, pain concerns decreased across the lifespan; however, there was a trend towards the ELBWs exhibiting an increase in pain at 30 to 35 years of age. This may reflect possible differences in physical pain that emerge as the ELBW survivors approach middle age.

\section{P93}

\section{ARE POSTAL SURVEYS STILL EFFECTIVE TO ASSESS ATTITUDES, PRACTICES AND EDUCATIONAL NEEDS OF PHYSICIANS?}

(Regular Research Poster)

Nancy Julien ${ }^{1}$, Huguette St-Amant ${ }^{1}$, Mark Ware ${ }^{2}$, Anaïs Lacasse ${ }^{1}$ Département des sciences de la santé, Université du Québec en Abitibi-Témiscamingue, Rouyn-Noranda; ${ }^{2}$ The Alan Edwards Pain Management Unit, McGill University Health Centre, Montreal, Quebec

AIM: Obtaining high response rates to postal surveys of physicians remains a challenge. Recent postal surveys conducted among Canadian physicians achieved $14.1 \%$ to $65.8 \%$ response rates. We aimed to describe strategies and response rates obtained during a study conducted among physicians in a northern region of the province of Quebec.

METHODS: In 2013, a postal survey about cannabinoid prescription for the management of chronic non-cancer pain was sent to all physicians in the region of Abitibi-Témiscamingue $(n=318)$. We used an adapted Dillman Total Design Method, which implies four mailing rounds: the initial mailing of a cover letter, the questionnaire and a stamped return envelope; a reminder postcard one week after the initial mailing; and questionnaire duplicates three and seven weeks after the initial mailing (we did not use registered mail for the fourth mailing). Other strategies included: a cover letter with a blue handwritten signature and direct telephone contact from the principal investigator, personalized correspondences, a $<12$ page colour-printed respondent-friendly questionnaire, and a teaser sentence on the mailing envelope.

RESULTS: A total of 166 physicians responded to the survey (family physicians: $56.0 \%$ ). Responders and non-responders were similar regarding medical specialty (family physicians: $56.0 \%$ versus $57.2 \%$ ) and sex (women: $53.6 \%$ versus $46.7 \%$ ). Individual response rates to each of the mailing rounds were respectively: $15.7 \%, 15.4 \%, 13.2 \%, 7.9 \%$. Cumulative response rates following these mailing rounds were respectively: $15.7 \%$, $31.1 \%, 44.3 \%, 52.2 \%$.

CONCLUSIONS: Although we cannot identify which of our strategies is the most effective in enhancing physician response rates, a substantial response rate was nevertheless achieved without the use of registered mail or monetary incentives.

\section{P94}

ILLNESS PERCEPTIONS MEDIATE THE RELATIONSHIP BETWEEN PAIN AND DEPRESSION IN WOMEN WITH IC/BPS Laura Katz ${ }^{1}$, Dean A Tripp ${ }^{1,2,3}$, Curtis J Nickel ${ }^{2}$, Adrijana Krsmanovic ${ }^{1}$, Abigail Muere $^{1}$, Lesley K Carr ${ }^{4}$, Robert Mayer ${ }^{5}$

${ }^{1}$ Department of Psychology; ${ }^{2}$ Department of Urology; ${ }^{3}$ Department of Anesthesiology, Queen's University, Kingston; ${ }^{4}$ Sunnybrook Health Sciences Centre, Toronto, Ontario; ${ }^{5}$ University of Rochester, Rochester, New York, USA

AIM: Interstitial Cystitis / Bladder Pain Syndrome (IC/BPS) is a chronic pelvic pain syndrome associated with pain, urological symptoms and disability (Nickel et al., 2010), and pain is a robust predictor of depression in women with IC/BPS (Tripp et al., 2012; Katz et al., 2012). Illness perceptions (IP) have been identified as important precursors to depression, but 
this has yet to be evaluated in chronic pelvic pain. The aim of this study is to evaluate IP as mediators on the relationship between pain and depression.

METHODS: Female patients with IC/BPS $(n=79)$ from urology clinics completed questionnaires (McGill Pain Questionnaire, Brief-Illness Perceptions Questionnaire, Patient Health Questionnaire-9). Multiple mediation modeling was used to examine the effects of three components of IP (cognitive, emotional and comprehension) on the relationship between pain and depression.

RESULTS: The omnibus effect of the three components of IP were significant in mediating the relationship between pain and depression $(R 2=0.48$, $F(4,79)=16.96, P<0.01)$. More specifically, indirect effects show that cognitive $(Z=0.10, P=0.05)$ and emotional $(Z=0.13, P=0.01)$ IP (but not illness comprehension) produced a significant partial mediation effect.

CONCLUSIONS: Results from this study show that IP were significant in mediation the relationship between pain and depression. Cognitive (eg, perceptions of how much your illness affects your life, control over your illness) and emotional (eg, concerns and emotional upset from illness) IP may be important precursors to depression in women with IC/BPS. Understanding these illness schemas is essential to developing psychological treatments in order to decrease depression and improve patient quality of life.

\section{P95}

\section{SURGICALLY PLACED ABDOMINAL WALL CATHETERS IMPROVE POSTOPERATIVE PAIN MANAGEMENT AFTER LIVE LIVER DONOR RESECTIONS}

James Khan $^{1,2}$, Joel Katz ${ }^{1,2}$, Salima Ladak ${ }^{1,2}$, Stuart McCluskey ${ }^{1,2}$, Coimbatore Srinivas ${ }^{1,2}$, Raynauld Ko ${ }^{1,2}$, David Grant ${ }^{2,3}$, Hance Clarke ${ }^{1,2}$

${ }^{1}$ Department of Anesthesia and Pain Management, Toronto General Hospital, ${ }^{2}$ University Health Network; ${ }^{3}$ Multi-organ Transplant Program, Toronto General Hospital, Toronto, Ontario

AIM: Liver resections utilizing a subcostal or chevron incision which produces moderate to severe postoperative pain. Epidural analgesia is the gold standard for postoperative pain management, but it is often refused or contraindicated. The aim was to conduct a retrospective chart review to compare abdominal wall catheters (AWC) plus intravensous (IV) patient controlled analgesia (PCA) with IV PCA or epidural analgesia on postoperative pain and recovery after live liver donor surgery.

METHODS: Following REB approval, a retrospective review was performed on 319 live liver donors. Sixty-eight patients received a thoracic epidural, 78 bilateral AWC plus IV PCA, and 171 IV PCA alone. One catheter was placed between the internal oblique and transversus abdominis fascia and the other between the rectus muscle and the posterior sheath.

RESULTS: Morphine consumption was significantly lower in the AWC group $(17.5 \pm 1.68 \mathrm{mg})$ vs the PCA group $(24.0 \pm 2.04 \mathrm{mg})$ on day 2 $(\mathrm{P}=0.03)$ and pain scores were lower on day 1 . Pain scores were significantly lower in the epidural group compared to the AWC group during some, but not all time periods. There were no group differences in time to drinking fluids, full diet, or ambulation among TAP and epidural or PCA groups. Length of stay (days) was significantly $(\mathrm{P}=0.007)$ shorter for the TAP $(5.95 \pm 0.128)$ vs PCA $(6.44 \pm 0.125)$ and epidural $(6.63 \pm 0.215)$ groups.

CONCLUSIONS: AWC provide better postoperative pain management compared to PCA. TAP catheters potentially improve postoperative recovery and reduce time to hospital discharge. Prospective trials are needed to verify these benefits.

\section{P96}

PSYCHOTHERAPEUTIC FACTORS AND TREATMENT OUTCOMES IN CHRONIC PAIN REHABILITATION

\section{John Kowal ${ }^{1}$, Keith G Wilson ${ }^{1}$, Peter R Henderson ${ }^{1}$,} Lachlan A McWilliams²

1The Ottawa Hospital Rehabilitation Centre, Ottawa, Ontario; ${ }^{2}$ University of Saskatchewan, Saskatoon, Saskatchewan

AIM: Interdisciplinary, group-based chronic pain management programs are effective for enhancing patients' functioning. In other areas of group therapy research, non-specific psychotherapeutic factors have been found to play an important role in mediating treatment outcomes. This study examined relationships among psychotherapeutic process variables and treatment outcomes for chronic pain.

METHODS: Consecutive patients $(\mathrm{n}=250)$ admitted to an interdisciplinary, group-based, outpatient, chronic pain management program at a rehabilitation hospital were recruited. They completed a battery of questionnaires at pre- and post-treatment. This included measures of pain intensity, functional limitations, pain catastrophizing, self-efficacy, fear of re-injury, depressive symptoms, and overall distress. At post-treatment, they also completed the Therapeutic Factors Inventory-Short Form, a wellvalidated 23 -item scale that assesses four factors relevant to successful outcome in psychotherapy (viz., the instillation of hope, secure emotional expression, awareness of interpersonal impact, and social learning).

RESULTS: In a series of hierarchical multiple regression analyses, the instillation of hope was significantly associated with decreased catastrophizing $(b=-0.27 ; \mathrm{P}=0.003)$, depressive symptoms $(\mathrm{b}=-0.41 ; \mathrm{P}<0.001)$, and overall distress $(b=-0.20 ; P=0.023)$, as well as increased pain self-efficacy $(b=0.23 ; P=0.015)$, after controlling for age, gender, and pre-treatment levels of the variable of interest. None of the therapeutic factors were associated with pain intensity.

CONCLUSIONS: Psychotherapeutic factors, most notably the instillation of hope, were associated with treatment outcomes following chronic pain rehabilitation. The findings highlight the importance of the therapeutic process, suggesting that enhanced outcomes can be derived by attending to these factors in the design and delivery of interdisciplinary, group-based programs.

\section{P97}

PSYCHOSOCIAL MEDIATORS OF THE PAIN-DEPRESSION RELATIONSHIP IN CP/CPPS

Adrijana Krsmanovic ${ }^{1}$, Dean A Tripp $p^{1,2,3}$, J Curtis Nickel ${ }^{3}$, Laura Katz ${ }^{1}$, Abigail Muere ${ }^{1}$, Daniel Shoskes ${ }^{4}$, Michel Pontari ${ }^{5}$, Mark S Litwin ${ }^{6}$, Mary F McNaughton-Collins ${ }^{7}$

${ }^{1}$ Psychology; ${ }^{2}$ Anesthesiology; ${ }^{3}$ Urology, Queen's University, Kingston, Ontario; ${ }^{4}$ Urology, Glickman Urological and Kidney Institute, Cleveland, Ohio, USA; ${ }^{5}$ Urology, Temple University, Philadelphia, Pennsylvania; ${ }^{6}$ Urology, David Geffen School of Medicine at UCLA, Los Angeles, California; ${ }^{7}$ Massachusetts General Hospital, Boston, Massachusetts, USA

AIM: Chronic Prostatitis/Chronic Pelvic Pain Syndrome (CP/CPPS) is a prevalent pelvic pain condition characterized by pain in the perineum, pelvic area and/or genitalia, and is refractory to medical interventions. Patients with CP/CPPS report higher depression rates than matched controls (Clemens et al., 2008), and higher depression scores predict greater overall pain (Tripp et al., 2004). The present study aimed to identify pain appraisals and behavioural coping strategies as mediators of the paindepression relationship, in order to better understand the CP/CPPS patient pain experience.

METHODS: A total of 175 men diagnosed with CP/CPPS were recruited from tertiary care urology clinics and completed packages consisting of demographic questions (age, education, ethnicity, employment status), and questionnaires on pain (Short-Form McGill Pain Questionnaire), depression (Center for Epidemiologic Studies Depression Scale), control over pain (Survey of Pain Attitudes - Control), catastrophizing (Pain Catastrophizing Scale), and behavioural coping (Chronic Pain Coping Inventory).

RESULTS: The group effect of psychosocial variables (illness-focused behavioural coping, wellness-focused behavioural coping, other behavioural coping, control over pain, catastrophizing) produced a full 
mediation effect $(\mathrm{R} 2=0.25, \mathrm{~F}(6,168)=9.25 ; \mathrm{P}<0.01)$ of the pain and depression relationship. Specific indirect effects show that catastrophizing is the only significant mediator $(Z=3.49 ; \mathrm{P}<0.01)$, with helpless catastrophizing specifically mediating this relationship $(Z=2.34 ; P<0.05)$.

CONCLUSIONS: Results suggest that helpless catastrophic thinking is the mechanism that explains the relationship between pain and depression in CP/CPPS patients. Targeting helplessness through psychotherapy interventions may reduce rates of depression in this patient population.

Footnotes/References: Clemens JQ, Brown SO, Calhoun EA. Mental health diagnoses in patients with interstitial cystitis/painful bladder syndrome and chronic prostatitis/chronic pelvic pain syndrome: A case/ control study. J Urol 2008;180:1378-82.

Tripp DA, Nickel JC, Landis JR, Wang YL, Knauss JS. Predictors of quality of life and pain in chronic prostatitis/chronic pelvic pain syndrome: findings from the National Institutes of Health Chronic Prostatitis Cohort Study. Br J Urol Int 2004;94:1279-82.

\section{P98}

\section{ARE GAS EXCHANGES MODIFIED IN CHRONIC PAIN} SUFFERERS?

Denis Laberge $^{1}$, Kadija Perreault ${ }^{2}$, Catherine Mercier ${ }^{2,3}$ ${ }_{1}^{1}$ IRDPQ, Chronic Pain Rehabilitation Expertise Program (Programme d'expertise de réadaptation en douleur chronique); ${ }^{2}$ Department of Rehabilitation, Université Laval, Laval; ${ }^{3}$ Centre interdisciplinaire de recherche en réadaptation et en intégration sociale (CIRRIS - Interdisciplinary Research Centre in Rehabilitation and Social Integration), Quebec, Quebec

AIM: Chronic pain sufferers may exhibit respiratory changes, which may influence their symptoms. For example, changes in respiratory movement patterns have been documented in individuals with chronic cervicalgia and low back pain. The aim of this literature review was to determine whether gas exchange measurement can objectify such respiratory changes. METHODS: Articles in French or English were searched in five databases (Pubmed, Embase, CINHAL, PsycInfo and Scopus), using the following key words: chronic pain, breathing, respiratory dysregulation, hyperventilation, hypocapnia, capnography, dyspnea. To be chosen, the studies had to measure the modification of gas exchanges (directly or indirectly) in human chronic pain sufferers. Animal studies and studies evaluating the effect of medications were excluded.

RESULTS: Nine studies were identified. The chronic conditions targeted in these studies were lumbar/lumbopelvic pain, cervicalgia, fibromyalgia and migraines/headaches. Eight studies dealt with the evaluation of gas exchanges and one with intervention. Most of the studies revealed a disturbance of the gas exchanges, translating into a decrease in carbon dioxide in chronic pain sufferers.

CONCLUSIONS: In light of the results obtained, disturbances of gas exchanges are found in different clienteles exhibiting chronic pain. However, given the few scientific articles obtained and their variable methodological qualities, additional studies are necessary on the subject.

\section{P99}

PERCEIVED CHRONIC PAIN MANAGEMENT SELF-EFFICACY: VALIDITY OF A FRENCH-CANADIAN SCALE

Anaïs Lacasse ${ }^{1}$, Patricia Bourgault ${ }^{2,3}$, Roxanne CourtemancheHarel $^{3}$, Manon Choinière ${ }^{4,5}$

${ }^{1}$ Département des sciences de la santé, Université du Québec en Abitibi-Témiscamingue, Rouyn-Noranda; ${ }^{2}$ École des sciences infirmières, Faculté de médecine et des sciences de la santé, Université de Sherbrooke; ${ }^{3}$ Centre de recherche clinique Étienne-Le Bel, Centre hospitalier universitaire de Sherbrooke, Sherbrooke; ${ }^{4}$ Département d'anesthésiologie, Faculté de médecine, Université de Montréal; ${ }^{5}$ Centre de recherche du Centre hospitalier de I'Université de Montréal, Montreal, Quebec

AIM: Perceived self-efficacy is a non-negligible outcome when evaluating the efficacy of interventions for the self-management of chronic pain. We aimed to establish the validity of a French-Canadian Chronic Pain Selfefficacy Scale (FC-CPSES).
METHODS: The Chronic Disease Self-Efficacy Scale is a validated 33-item (or 6-item) self-administered questionnaire that measures selfefficacy to perform self-management behaviors, manage disease in general, and achieve outcomes (Lorig et al.,1996). We adapted this scale to the context of chronic pain patients and used a double back translation method. The FC-CPSES was administered to 109 fibromyalgia patients who participated in an evidence-based self-management intervention offered in nine healthcare centers of the province of Quebec (PASSAGE Program). Cronbach alpha coefficients $(\alpha)$ were calculated as the measures of the internal consistency of the 33- and 6-item versions of the FC-CPSES. With respect to construct validity, we measured the association between the FC-CPSES baseline scores and related clinical outcomes. We also compared pre- and post-intervention FC-CPSES scores.

RESULTS: Internal consistency was high for both versions of the FC-CPSES (33-item: $\alpha=0.93$; 6-item: $\alpha=0.86$ ). FC-CPSES scores were significantly associated with lower pain intensity, better mental quality of life, and greater coping strategies $(\mathrm{P}<0.05)$. There was a statistically significant increase in FC-CPSES scores between pre- and post-intervention measures for both versions of the FC-CPSES $(\mathrm{P}<0.003)$ which support their sensitivity to clinical changes during an intervention.

CONCLUSIONS: Our data suggest that both versions of the FC-CPSES are reliable and valid scales to measure chronic pain management selfefficacy among fibromyalgia patients.

\section{P100}

\section{CAN TDCS ENHANCE THE THERAPEUTIC EFFECT OF GRADED MOTOR IMAGERY IN PATIENTS PRESENTING CHRONIC CRPS TYPE I? PRELIMINARY RESULTS}

Emilie Lagueux $^{1}$, Laurence Beaulieu-Boire ${ }^{2}$,

Roxanne Courtemanche-Harel ${ }^{1}$, Patricia Bourgault ${ }^{3}$, Yannick Tousignant-Laflamme ${ }^{2}$

${ }^{1}$ Clinical Research Center Etienne-Le Bel, Centre hospitalier universitaire de Sherbrooke (CHUS); ${ }^{2}$ School of Rehabilitation, Faculty of Medicine and Health Sciences; ${ }^{3}$ School of Nursing, Faculty of Medicine and Health Sciences, Université de Sherbrooke, Sherbrooke, Quebec

AIM: Although graded motor imagery (GMI) has been shown to help reduce pain in complex regional pain syndrome (CRPS), chronic CRPS is a major challenge in rehabilitation. A potential solution to enhance clinical outcomes would be to add an electrotherapeutic procedure, such as transcranial direct current stimulation (tDCS). The goal of this clinical research project was to investigate the clinical efficacy of the combination of GMI and tDCS versus GMI combined with sham tDCS in patients presenting chronic CRPS.

METHODS: Data was collected from six participants presenting CRPS type I for a mean of 2.2 years. They were randomly assign to experimental $(\mathrm{GMI}+\mathrm{tDCS})$ or control (GMI + sham $\mathrm{tDCS})$ group. The GMI treatments were performed over six weeks using well-established procedures (www. noigroup.com). The tDCS was applied for five consecutive days during the first two weeks and once a week during the four following weeks. The outcome measures were assessed before and after the treatments: i) The global impression of change regarding pain, ii) Clinical pain intensity and overall physical function; iii) Quality of life; iv) Pain catastrophizing.

RESULTS: Patients in the experimental group reported greater impression of pain relief ( $13 \%$ versus $6 \%$ ) and a clinically significant reduction in clinical pain intensity and overall physical function (reduction $>2 / 40$ ). However, we observed no significant difference in quality of life and pain catastrophizing.

CONCLUSIONS: Although our preliminary results tend to show an enhanced clinical effect for patients that received the tDCS as an add-on treatment, the observed changes remain clinically marginal. 
P101

PAIN-QUILT $^{\mathrm{TM}}$ : COMPARISON OF A WEB-BASED METHOD FOR THE VISUAL EXPRESSION OF PAIN WITH THE MCGILL PAIN QUESTIONNAIRE AND BRIEF PAIN INVENTORY AMONG ADULTS WITH CHRONIC PAIN

Chitra Lalloo ${ }^{1}$, Dinesh Kumbhare ${ }^{2}$, James L Henry ${ }^{1}$

${ }^{1}$ Faculty of Health Sciences, McMaster University, Hamilton; ${ }^{2}$ University of Toronto, Toronto, Ontario

AIM: Pain-QuILT is a web-based tool for the visual expression and electronic tracking of pain quality, intensity, and location that can be tracked over time. Previous work has described content validation, usability, and clinical feasibility testing in adolescents and adults with chronic pain. This study compared Pain-QuILT with the McGill Pain Questionnaire (MPQ) and Brief Pain Inventory (BPI) in terms of: ease of use, time to complete, and patient preferences.

METHODS: Participants were recruited from a hospital-affiliated adult pain management and physical rehabilitation outpatient clinic in Southwestern Ontario. Participants self-reported their pain using PainQuILT, MPQ, and BPI (randomized order). A semi-structured interview was used to discuss preferences for pain self-report. A 0-10 scale ranging from "not easy at all" to "very easy" was used to rate each tool.

RESULTS: The sample was comprised of $n=50$ adults with chronic pain ( $54 \%$ female; mean age 50$)$. Mean $( \pm$ SD) scores for ease of use were: 5.9 \pm 2.6 for MPQ, $7.0 \pm 2.6$ for BPI, and $8.3 \pm 2.0$ for Pain-QuILT. Mean $( \pm \mathrm{SD})$ time required for tool completion was: $4.2 \pm 1.5 \mathrm{~min}$ for MPQ, 4.0 $\pm 1.4 \mathrm{~min}$ for BPI, and 4.1 $\pm 2.2 \mathrm{~min}$ for Pain-QuILT. Overall, participant preferences were: 59\% for Pain-QuILT, 16\% for MPQ, 14\% for BPI, 8\% for verbal report, and $2 \%$ for BPI and Pain-QuILT equally.

CONCLUSIONS: In comparison with two of the most commonly used pain assessment tools, data indicate that Pain-QuILT is: easier to use, comparable in terms of time to complete, and preferred by a majority of patients. A free demo is available at: http://painquilt.mcmaster.ca

\section{P102}

\section{EXPLORATION OF PHYSICIAN-SPECIFIC RELATIONSHIP FACTORS INFLUENCING MANAGEMENT OF CHRONIC PAIN PATIENTS}

Thi Thu Hang Le ${ }^{1}$, Marc Dumas ${ }^{2}$, Sylvie Lafrenaye ${ }^{3}$

${ }^{1}$ Faculty of Medicine and Health Sciences; ${ }^{2}$ Faculty of Theology and Religious Studies, Université de Sherbrooke; ${ }^{3}$ Université de Sherbrooke and CHU Sherbrooke, Sherbrooke, Quebec

AIM: Chronic pain is difficult, both for the care recipient and the caregiver. It is suggested that a person-focused approach is the best way to help these patients. However, little is known about how physicians adapt their practice to the failure represented by chronic pain. The goal is to explore the factors influencing physicians in a chronic pain context, in order to understand how their identity, spirituality and relationship characteristics orient their care approach.

METHODS: Qualitative research in grounded theory, based on the conceptual frameworks of the narrative identity (Ricœur) and of time (Tillich). Individual interviews were conducted with seven physicians in various specialties, four of whom were interviewed a second time to deepen the theme of identity.

RESULTS: Our approach allowed us to identify "recognition" as an emerging process to explain the progression of the physician's professional and personal identity. Although not researched explicitly, mutual recognition is a source of motivation and unsuspected energy to maintain a quality relationship. The French word "reconnaissance" (recognition) thus can be broken down into "re-co-naissance" (re-co-birth), the co-rebirth of a relationship to define oneself anew as a therapist and to not experience medical failure. CONCLUSIONS: The new relationship competence acquired, added to professional competence, allows individual physicians to enrich their knowledge but also to progress over time, moving from professional identity to spiritual recognition and a dynamic relationship recognition ("re-co-naissance") with their pain patient so that they no longer experience medical failure.

\section{P103}

USE OF DISTRACTION KITS FOR PEDIATRIC PROCEDURAL PAIN MANAGEMENT IN EMERGENCY: A PILOT STUDY

Sylvie Le May ${ }^{1,2}$, Sylvie Charette ${ }^{1}$, Marie-Claude Charest ${ }^{1}$, Edith Villeneuve ${ }^{1}$, Benoit Bailey ${ }^{1}$, Christelle Khadr ${ }^{3}$, Jacinthe Lachance Fiola ${ }^{4}$

${ }^{1} \mathrm{CHU}$ Ste Justine, ${ }^{2}$ University of Montreal; ${ }^{3}$ McGill University Health Centre; ${ }^{4}$ Université du Québec à Montréal, Montreal, Quebec

AIM: A recent retrospective study on procedural pain management showed that less than $30 \%$ of children received an intervention to control their pain prior to the procedure. Distress felt during these procedures, may increase anxiety and pain for subsequent procedures. This study aimed to evaluate the usability and effect of distraction kits on procedural pain management in the Emergency Department (ED).

METHODS: Pilot study with a pre-post design. Children between zero and five years of age ( 0 to 2 years and 3 to 5 years groups), presenting to the ED and requiring a needle-related procedure were recruited. Pain was evaluated with the FLACC scale and parents and nurses were required to respond to two questionnaires: Usability and Satisfaction related to the Distraction Kits (DK).

RESULTS: Overall, 51 patients/parents participated ( 26 in 0 to 2 years; 25 in 3 to 5 years) and 44 nurses. An average of two procedures were done per child. Children were mainly lying down during the procedure (96\%). The most utilized toy for the 0 to 2 group was the Propeller (lights/sound) $(85 \%)$ and the Bubbles for the 3 to 5 years $(68 \%)$. Satisfaction regarding the DK was $30.9 / 40(+5.0)$ for nurses and $32.4 / 40(+5.5)$ for parents. Pain score was $5.9+3.3$ (per-procedure) and 2.2 +2.9 (post-procedure).

CONCLUSIONS: The study showed that use of the DK in ED was feasible and a useful method of distraction. Parents and nurses suggest to use toys that produce sounds and lights, and also to use applications (DVD or iPad) with same features.

\section{P104}

\section{ASSESSING PAIN: THE DEAF COMMUNITY EXPERIENCE}

Sandra LeFort ${ }^{1}$, Victor Maddelena ${ }^{2}$, Myles Murphy ${ }^{3}$

${ }^{1}$ School of Nursing; ${ }^{2}$ Faculty of Medicine, Memorial University;

${ }^{3}$ Newfoundland and Labrador Association for the Deaf, St John's,

Newfoundland and Labrador

AIM: There is little research examining Deaf peoples perceptions of pain and their ability to communicate their pain to others. Two studies $(1,2)$ have investigated the problem of pain in the Deaf at end-of-life and palliative care and one study (3) assessed four uni-dimensional pain scales with Deaf patients in a general hospital environment. These three studies identified similar concerns expressed by Deaf patients in relation to pain assessment and communication. The challenges are compounded by the need for interpreters who translate American Sign Language (ASL) into English when the Deaf seek medical treatment. The purpose of this study was to examine Deaf peoples perceptions of pain and its communication, the ASL interpreters experience with communicating pain from the Deaf person to a health provider, and the best standardized pain intensity assessment tool for use with Deaf people.

METHODS: Focus groups with Deaf people and ASL interpreters were conducted using audio recordings and videotaping to better understand their perspectives in pain assessment with health care providers. In addition, a new faces-type pain scale that attempts to be more appropriate to the Deaf population was developed. The new scale was compared to other commonly used uni-dimensional pain scales (eg, Iowa Pain Thermometer Scale, Wong-Baker FACES Pain Rating Scale, the 0-10 scale, etc). IREB approval by the provincial ethics board was obtained.

RESULTS: ASL interpreters $(n=4)$ and Deaf people $(n=7)$ reported multiple issues related to pain communication. The interpreters were often challenged to appropriately represent the perspective of the Deaf person to the health care provider and described the complexity of pain communication. Both the interpreters and Deaf people were comfortable in describing the location of the pain and its impact on function. However, descriptions of intensity and what the pain feels like proved difficult. When asked to compare various standard pain tools with the new Deaf faces tool, 
participants appeared to prefer the new tool which included six expressive faces of a Deaf man and a Deaf woman with numerical ratings from 0 to 5 with colour gradients.

CONCLUSIONS: Pain assessment in the Deaf is a neglected area of study. The results of this pilot qualitative project highlight the concerns and difficulties that Deaf people and their ASL interpreters face in communicating pain in health care settings. A first attempt at designing a more Deaf-appropriate pain assessment tool may be useful for assessing pain in the Deaf community. More research needs to be done.

Footnotes/references:

1. Maddelena V, OShea F, Murphy M Palliative and end-of-life care in Newfoundlands Deaf community. J Palliative Care, 2012;28:105-12.

2. Allen B, Meyers N, Sullivan J, Sullivan M American sign language and end-of-life care: Research in the Deaf community. HEC Forum 2002;14:197-208.

3. Palese A, Salvador L, Cozzi D. One-dimensional scales for pain evaluation adopted in Italian nursing practice: Giving preference to Deaf patients. J Nurs Meas 2011;19:91-104.

\section{P105}

THE RELATIONSHIP BETWEEN PARENT PAIN MANAGEMENT TECHNIQUES AND INFANT PAIN-RELATED DISTRESS

Diana Lisi ${ }^{1}$, Lauren Campbell ${ }^{1}$, Rebecca Pillai Riddell ${ }^{1,2,3}$,

Hartley Garfield ${ }^{2,3}$, Saul Greenberg ${ }^{2,3}$

${ }^{1}$ York University; ${ }^{2}$ The Hospital for Sick Children; ${ }^{3}$ University of Toronto, Toronto, Ontario

AIM: To examine the relationships between pharmacological and nonpharmacological pain management techniques and infant pain-related distress.

METHODS: Caregivers and infants were part of a longitudinal cohort (the OUCH cohort) and were followed and videotaped during their two-, four-, six-, and 12-month immunizations $(\mathrm{n}=760)$. Videotapes were coded for non-pharmacological pain management techniques (MAISD; Cohen et al., 2005) and infant pain-related distress (NFCS; Grunau \& Craig, 1987). Parents provided self-report as to whether they had administered pharmacological analgesics to their infants (ie, EMLA, Tylenol).

RESULTS: Across all four ages, the use of pharmacological techniques was minimal. Given their limited use, pharmacological techniques were not included in any subsequent analyses. Controlling for preceding infant pain levels, parent non-pharmacological pain management techniques accounted for, at most, $13 \%$ of the variance in infant pain scores. Multiple regression analyses showed that pacifying and distraction were negatively related to pain scores at two and six months, respectively. All other predictors positively predicted infant pain scores.

CONCLUSIONS: Parent non-pharmacological pain management techniques appear to minimally predict infant pain-related distress over the first year of life. Specific strategies known to reduce pain (eg, EMLA) were the most infrequently used strategies. Despite the lack of direct efficacy on lowering infant pain-related distress, it is crucial to continue to underscore the importance of non-pharmacological soothing behaviors when an infant is in distress. The relationships between parental soothing of infant distress and more psychosocial measures (such as the formation of a secure attachment bond) remain imperative to an infant's emotional development.

\section{P106}

\section{A LONGITUDINAL, DESCRIPTIVE EXAMINATION OF NATURALLY OCCURRING PAIN MANAGEMENT TECHNIQUES CURRENTLY USED IN INFANT IMMUNIZATIONS}

Diana Lisi ${ }^{1}$, Lauren Campbell ${ }^{1}$, Rebecca Pillai Riddell ${ }^{1,2,3}$, Hartley Garfield $^{2,3}$, Saul Greenberg ${ }^{2,3}$, Priyanjali Mithal ${ }^{1}$ ${ }^{1}$ York University; ${ }^{2}$ The Hospital for Sick Children; ${ }^{3}$ University of Toronto, Toronto, Ontario

AIM: To provide descriptive information about naturally occurring pain management techniques currently used during the first year of life during infant immunizations.
METHODS: Caregivers and infants were part of a longitudinal cohort (the OUCH cohort) and were followed and videotaped during their two-, four-, six-, and 12-month immunizations $(\mathrm{n}=760)$. Videotapes were coded for parent soothing behaviors (MAISD; Cohen et al., 2005).

RESULTS: Pharmacological techniques: Across all four ages, the use of pharmacological techniques was minimal (with Tylenol/Tempra used 6.9\% of the time at two months and $11.7 \%$ of the time at four months, and EMLA used $<1 \%$ of the time at all ages). Non-pharmacological techniques: Across all ages and 1 min periods post-needle, physical comfort, rocking, and verbal reassurance were the most commonly occurring behaviours (between $8 \%$ and $47.5 \%$ of the time). There was a $10 \%$ decrease in physical comfort and rocking from two to 12 months of age, at both 1 min and $2 \mathrm{~min}$ post-needle, and $2 \mathrm{~min}$ and $3 \mathrm{~min}$ post-needle, respectively. Regardless of age, offering food, offering a toy, and nursing were the least frequently used techniques $(<5 \%$ of the time).

CONCLUSIONS: Overall, pharmacological techniques to reduce pain are not being used by parents, despite previous research supporting their effectiveness. Comparatively, parents used non-pharmacological techniques much more often. These findings provide a framework in which we can begin to design and implement strategies to encourage parents to use evidence-based pain reduction techniques.

\section{P107}

\section{PLASMIN: A NOVEL THERAPEUTIC TARGET FOR THE TREATMENT OF NEUROPATHIC PAIN}

Claire Magnussen, Francisney Nascimento, A Claudio Cuello, Alfredo Ribeiro-da-Silva

Department of Pharmacology and Therapeutics and Alan Edwards Centre for Research on Pain, McGill University, Montreal, Quebec AIM: Nerve growth factor (NGF) is essential for the development and maintenance of sensory and sympathetic fibers but is also a pain mediator. NGF is secreted from cells in its precursor form (proNGF) and is converted to mature-NGF by the enzyme plasmin (Bruno and Cuello, PNAS, 2006). Alterations in NGF levels are thought to underlie the abnormal sensory and sympathetic innervation in the skin and the pain in neuropathic models. The aim of this study was to assess the effects of plasmin inhibition on 1) pain-related behaviour 2) skin innervation 3) NGF levels in a mental nerve chronic constriction injury (CCI) model.

METHODS: CCI rats received daily subcutaneous injections of vehicle or $1 \mu \mathrm{g} / 20 \mu \mathrm{L}, 8 \mu \mathrm{g} / 20 \mu \mathrm{L}$ or $20 \mu \mathrm{g} / 20 \mu \mathrm{L}$ of $\alpha 2$-antiplasmin (plasmin inhibitor), into the skin of the lip for two weeks, starting immediately after surgery. Mechanical allodynia was tested by von Frey and lip skin was processed by immunohistochemistry using antibodies against calcitonin gene-related peptide (CGRP) and dopamine beta hydroxylase (DBH), labeling peptidergic and sympathetic fibers, respectively. Western blotting was used to measure levels of pro- and mature-NGF.

RESULTS: The significant sensitization to mechanical stimuli caused by CCI was prevented by antiplasmin $(8 \mu \mathrm{g}$ and $20 \mu \mathrm{g})$. The reduction of CGRP sensory fibers was normalized by $8 \mu$ antiplasmin, only. Surprisingly, there was a dose-dependent sprouting of sympathetic fibers into the upper dermis of the skin, a region where they are not normally found.

CONCLUSIONS: Plasmin could represent a novel therapeutic target for treating the symptoms of neuropathic pain.

\section{P108}

\section{PARENTS' AND NURSES' PERSPECTIVES OF INFANTS', PRESCHOOLERS', AND PREADOLESCENTS' INJECTION DISTRESS}

Sarah Martin, Lindsey Cohen

\section{Georgia State University, Atlanta, Georgia, USA}

AIM: Children's self-report is central in pain assessment; however, parents' and healthcare providers' reports are also critical. Unfortunately, research has revealed inconsistency in perspectives. We aim to examine associations among adult report and child report of distress during immunizations in infants, preschoolers, and preadolescents.

METHODS: Participants included 82 infants (mean age 32 weeks; $47 \%$ female), 29 preschoolers (mean age 4.7 years; $42 \%$ female), 
and 33 preadolescents (mean age 10 years; 58\% female) undergoing immunizations.

RESULTS: Parent and nurse report of infant distress were correlated $(\mathrm{rs}=0.24 ; \mathrm{P}=0.07)$ and both were associated with observational distress $(\mathrm{rs}=0.24 ; \mathrm{P}=0.04)$. Parents' report of their own distress was significantly associated with their report of infant distress ( $r s=0.34 ; \mathrm{P}<0.01)$. With preschoolers, parent report was higher than nurse-report $(z=-2.50 ; \mathrm{P}=0.04)$ and no differences existed between child self-report and nurse- or parentreport. Parents' own distress was significantly associated with parent-report of preschooler distress $(\mathrm{rs}=0.53 ; \mathrm{P}<0.01)$. With preadolescents, nursereport was significantly higher than child self-report $(\mathrm{Z}=-1.48 ; \mathrm{P}=0.02)$ and nurse-report of child distress was associated with nurses' own distress during the injection $(\mathrm{rs}=0.35 ; \mathrm{P}=0.049)$.

CONCLUSIONS: Results suggest that parent- and nurse-report of pediatric injection distress is more inter-correlated with infants than with older patients, which might be due to distress becoming less overt with age. Further, adults' own distress during pediatric injections appears to influence their perception of the patient's pain.

\section{P109 \\ SOMATIZATION AND PAIN IN PATIENTS IN A PEDIATRIC EMERGENCY DEPARTMENT}

Rika Meyer, Iris Beltran Gonzalez, Jeffrey Gold

Children's Hospital of Los Angeles/University of Southern

California, Los Angeles, California, USA

AIM: We examined whether level of global pain experienced in the past week and chronicity of the presenting complaint predicted somatization in patients receiving services in a pediatric emergency department. Gender differences were also examined.

METHODS: Ninety-nine participants ( 53 girls, 46 boys; eight to 18 years of age) and their caregivers were recruited from the pediatric emergency department at an urban hospital. Parents were asked whether the patients' complaint was chronic (persisted the past three months). Patients were also asked to rate pain using a $10 \mathrm{~cm}$ visual analogue scale ranging from "no pain" to "worst pain imaginable." Somatization was assessed using the Children's Somatization Inventory (CSI).

RESULTS: The patient's chronic status significantly predicted increased somatization ( $b=11.51, \mathrm{SE}=2.63 ; \mathrm{P}=0.00$ ). Higher levels of subjective pain in the past week significantly predicted more somatization $(b=0.95$, $\mathrm{SE}=0.37 ; \mathrm{P}=0.01$ ). No significant interaction was found between chronicity and pain on somatization. Eighteen percent boys and $18 \%$ girls had a chronic complaint and $28 \%$ boys and $35 \%$ girls did not have a chronic complaint $(\times 2(1)=0.28 ; P=0.59)$. There were no significant gender differences in somatization $(\mathrm{t}(97)=-0.03 ; \mathrm{P}=0.97)$ and pain $(\mathrm{t}(97)=0.41$; $\mathrm{P}=0.69$ ).

CONCLUSIONS: Results suggest that for children who present to the $\mathrm{ED}$, regardless of gender, pain experienced in the past week and chronicity of condition significantly predicted higher levels of somatization. It is important for future research to examine the mechanisms through which global level of pain and persistence of a medical condition impact children's somatization symptoms.

\section{P110 \\ COPING IN CHILDREN AND ADOLESCENTS WITH PRIMARY AND SECONDARY PAIN DISORDERS}

Rika ML Meyer, Katie Fleischman, Jeffrey I Gold Children's Hospital of Los Angeles, Los Angeles, California, USA

AIM: We examined group differences in patients with primary (eg, arthritis) versus secondary (eg, cancer pain) pain disorders on type of coping (eg, primary control - eg, problems solving, secondary control - eg, distraction, and disengagement coping - eg, avoidance).

METHODS: Patients seeking pain management services for chronic pain complaints at a multidisciplinary outpatient clinic in an urban children's hospital were screened for participation. Fifty-five children and adolescents (eight to 18 years of age; Mage $=13.48, \mathrm{SD}=2.78$ ) with chronic pain and their parents completed measures assessing type of pain disorder. The Responses to Stress Questionnaire, a measure on coping and involuntary responses to stress associated with pain was completed. Pain was assessed with a $10-\mathrm{cm}$ Visual Analog Scale ranging from "no pain" to "worst pain imaginable."

RESULTS: A significant difference was found between patients with a primary versus secondary pain disorder on the parent's report of disengagement coping $(\mathrm{t}[46.51]=-2.51 ; \mathrm{P}=0.02)$. Those with a secondary pain disorder displayed more disengagement coping compared to those with a primary pain disorder. Additionally, there was a significant difference in parent's report of most pain experienced, where those with a primary pain disorder had more pain than those with a secondary pain disorder $(\mathrm{t}[53]=$ $-2.04 ; \mathrm{P}=0.04)$.

CONCLUSIONS: Understanding the implications of children with various chronic pain conditions coping behaviors can aid in the development of specific therapeutic interventions.

\section{P111}

\section{ACUPUNCTURE SATISFACTION IN CHILDREN AND ADOLESCENTS WITH CHRONIC PAIN}

Rika ML Meyer, Monika Kobylecka, Jeffrey I Gold

Children's Hospital Los Angeles, Los Angeles, California, USA

AIM: We examined patient satisfaction of acupuncture provided for children and adolescents with chronic pain.

METHODS: A sample of 239 children and adolescents (M[age] $=15.81$, $\mathrm{SD}=5.08)$ receiving acupuncture for the first time at a pediatric pain clinic participated. Participants completed a brief satisfaction questionnaire immediately after treatment using $10-\mathrm{cm}$ visual analogue scales ranging from 0 indicated "strongly disagree" to 10 indicated "strong agree." The FACES Pain Scale Revised (FPS-R) was also used to assess pain intensity. RESULTS: Patients rated whether they thought acupuncture was "good" $(\mathrm{M}=8.36, \mathrm{SD}=2.22)$, whether acupuncture helped them to feel "better and relaxed" $(M=8.20, S D=2.30)$, and whether acupuncture helped them to feel "less anxious" $(\mathrm{M}=7.68, \mathrm{SD}=2.64)$. One hundred sixty seven patients reported that acupuncture decreased their pain $(67.7 \%)$, eight $(3.4 \%)$ reported that it did not decrease pain, and 67 (29\%) reported "not sure" if acupuncture decreased pain. Patients reported minimal pain associated with acupuncture needling $(\mathrm{M}=1.38$ out of $10, \mathrm{SD}=2.02)$ using the FPS-R. Finally, patients reported high satisfaction with their acupuncturist $(\mathrm{M}=9.11, \mathrm{SD}=5.08)$.

CONCLUSIONS: Overall, patients were satisfied with their first treatment, reported minimal pain associated with acupuncture, and positive outcomes. Acupuncture may be a feasible and useful treatment for children and adolescents with chronic pain.

\section{P112}

VALIDATION OF THE NECKPIX@: A PICTORIAL INSTRUMENT FOR RATING FEAR OF MOVEMENT IN ACTIVITIES OF DAILY LIVING IN NECK PAIN PATIENTS Marco Monticone $^{1,2}$, Howard Vernon ${ }^{3}$, S Ferrante ${ }^{1}$, R Brunati $^{1}$, B Rocca ${ }^{1}$

${ }^{1}$ Physical Medicine and Rehabilitation Unit, Scientific Institute of Lissone, Milan; ${ }^{2}$ Institute of Care and Research, Salvatore Maugeri Foundation, IRCCS, Pavia, Italy; ${ }^{3}$ Canadian Memorial Chiropractic College, Toronto, Ontario

AIM: Development and validation of a set of neck-stressing images (NeckPix@) to assess fear-of-movement related to neck pain (NP).

METHODS: NP-related activities were reviewed from several validated sources and a final set of 10 items was developed by means of item generation and item reduction/selection. These were converted into images: the NeckPix@ collection. 50 adult males and females with chronic mechanical NP ( $>3$ months) were included in the validation study. Subjects rated their expectation of pain for each item on a 0-10 scale. They also provided data about the content validity of the items (including face validity, target population, relevance and completeness) and the feasibility of the scale. After summarizing clinical and demographic data, internal consistency (Cronbach's alpha) and test-retest reliability (Intraclass Correlation Coefficient [ICC]) were assessed; construct validity was investigated by means of Pearson's correlations between the NeckPix and the Italian 
versions of the Neck Disability Index (NDI), a pain severity scale (NRS), the Pain Catastrophising Scale (PCS), and the Tampa Scale for Kinesiophobia (TSK).

RESULTS: 50 subjects (36 females) of mean age 46.2(15.1) participated. The mean NeckPix@ score was $48.9 \%(17.7)$. Participants found the NeckPix@ to be highly appropriate and feasible. Cronbach's alpha was 0.89 [0.84-0.93] and ICC was 0.982 [0.968-0.990]. Correlations with the other instruments ranged from 0.542 (NDI) to 0.657 (NRS). No floor or ceiling effects were noted.

CONCLUSIONS: The NeckPix@ has good internal psychometrics and is fair-moderately highly correlated with other pain-related measures, indicating that it makes a distinct contribution to the analysis of chronic NP.

\section{P113}

DEMOGRAPHIC AND TREATMENT FACTORS RELATED TO ANALGESIA USE POST MAJOR LOWER LIMB AMPUTATION Janice Montbriand $^{1,2}$, Oliver GS Ayling ${ }^{3}$, Jiao Jiang1, Salima Ladak', Lindsay Love ${ }^{3}$, Naomi Eisenberg ${ }^{3}$, Hance Clarke ${ }^{2,4}$, Joel Katz ${ }^{2,4,5}$, Graham Roche-Nagle ${ }^{3}$

${ }^{1}$ Department of Psychology, York University; ${ }^{2}$ Department of Anesthesia and Pain Management, Pain Research Unit, University Health Network, Toronto General Hospital, University of Toronto; ${ }^{3}$ Division of Vascular Surgery, Toronto General Hospital, Peter Munk Cardiac Centre, University Health Network, University of Toronto; ${ }^{4}$ Department of Anesthesia, University of Toronto; ${ }^{5}$ Department of Psychology, York University, Toronto, Ontario AIM: The aim of this study was to investigate demographic, medical and treatment factors related to opioid consumption after a major lower limb amputation. This includes an investigation of whether there is a morphinesparing effect seen in the use of perineural stump catheter after controlling for major medical and demographic variables.

METHODS: 198 participants, having had lower limb amputation at the Toronto General Hospital, were identified through a retrospective chart review. Multiple regression analyses were used to build a model, identifying variables important in predicting post-operative morphine consumption during the $72 \mathrm{~h}$ post-surgical period.

RESULTS: In the final model, four variables related to total $72 \mathrm{~h}$ morphine use. A previous history of chronic pain and use of patient-controlled analgesia were associated with an increase in morphine use, while increasing age and the use of a perineural stump catheter were associated with a morphine- sparing effect.

CONCLUSIONS: The use of a perineural stump catheter was associated with a morphine-sparing effect during the first $72 \mathrm{~h}$ post-operative period after major lower limb amputation. Increasing age was associated with a smaller decrease in morphine use, while previous chronic pain was a risk factor for higher morphine use. This suggests that further research into the use of perineural stump catheter is warranted.

\section{P114}

PHARMACOLOGICAL MANAGEMENT OF CHRONIC NEUROPATHIC PAIN - REVISED CONSENSUS STATEMENT FROM THE CANADIAN PAIN SOCIETY

DE Moulin ${ }^{1}$, A Boulander ${ }^{2}$, AJ Clark ${ }^{3}$, H Clarke ${ }^{4}$, T Dao ${ }^{5}$, GA Finley $^{6}$, A Furlan ${ }^{7}$, A Gilron ${ }^{8}$, A Gordon ${ }^{9}$, PK Morley-Forster ${ }^{10}$, BJ Sessle ${ }^{9}$, P Squire ${ }^{11}$, J Stinson ${ }^{12}$, P Taenzer ${ }^{13}$, C Toth $^{14}$, A Velly ${ }^{15}$, MA Ware ${ }^{16}$, EL Weinberg ${ }^{17}$, OD Williamson ${ }^{18}$

${ }^{1}$ Departments of Clinical Neurological Sciences \& Oncology, Western University, London, Ontario; ${ }^{2}$ Anesthesiology, Montreal University, Montreal, Quebec; ${ }^{3}$ Anesthesia, Pain Management and Perioperative Medicine, Dalhousie University, Halifax, Nova Scotia; ${ }^{4}$ Department Anesthesia; ${ }^{5}$ Faculty of Dentistry, University of Toronto, Toronto, Ontario; ${ }^{6}$ Anesthesia \& Psychology, Dalhousie University, Halifax, Nova Scotia; ${ }^{7}$ Division of Physiatry, Department of Medicine, University of Toronto, Toronto; ${ }^{8}$ Anesthesiology \& Perioperative Medicine, Queen's University, Kingston; ${ }^{9}$ University of Toronto, Toronto; ${ }^{10}$ Department Anesthesiology \& Perioperative Medicine, Western University, London, Ontario; ${ }^{11}$ University of British Columbia, Vancouver, British Columbia; ${ }^{12}$ Lawrence S Bloomberg Faculty of Nursing, University of Toronto, Toronto, Ontario; ${ }^{13}$ Departments of Psychiatry, Medicine \& Oncology; ${ }^{14}$ Department of Neurosciences, University of Calgary, Calgary, Alberta; ${ }^{15}$ Faculty of Dentistry; ${ }^{16}$ Anesthesia and Family Medicine, McGill University, Montreal, Quebec; ${ }^{17}$ Toronto, Ontario; ${ }^{18}$ JPOCSC Pain Management Clinic, Fraser Health Authority, Surrey, British Columbia

AIM: To update the consensus statement from the Canadian Pain Society on the pharmacological management of neuropathic pain.

METHODS: Randomized, controlled trials, systematic reviews and existing guidelines focusing on the pharmacological management of $\mathrm{NeP}$ were evaluated at a consensus meeting in May 2012. Medications were recommended in the consensus statement if their analgesic efficacy was supported by at least one methodologically sound randomized, controlled trial (Class I or Class II) showing significant benefit relative to placebo or another relevant control group. Recommendations for treatment were based on degree of evidence of analgesic efficacy, safety, and ease of use. RESULTS: Analgesic agents recommended for first-line treatments are gabapentinoids (gabapentin and pregabalin), tricyclic antidepressants and serotonin noradrenaline reuptake inhibitors. Tramadol and controlledrelease opioid analgesics are recommended as second-line treatments for moderate to severe pain. Cannabinoids are now recommended as third-line treatments based on recent evidence of analgesic efficacy in multiple neuropathic conditions. Recommended fourth-line treatments include methadone, anticonvulsants with lesser evidence of efficacy (eg, lamotrigine, lacosamide), tapentadol and botulinum toxin. There is support for some analgesic combinations in selected neuropathic pain conditions.

CONCLUSIONS: Pharmacologic management of neuropathic pain remains challenging but an evidence-based stepwise approach optimizes outcome. Treatment should be individualized for each patient based on efficacy, side-effect profile and drug accessibility including cost. Further studies are required to examine head-to-head comparisons among analgesics, combinations of analgesics, long-term outcomes and treatment of pediatric, geriatric and central $\mathrm{NeP}$.

\section{P115}

\section{PSYCHOSOCIAL PREDICTORS OF MENTAL QUALITY OF} LIFE IN IC/BPS

Abigail Muere ${ }^{1}$, Dean A Tripp ${ }^{1,2,3}$, Jess Ginting1, J Curtis Nickel ${ }^{2}$, Laura Katz ${ }^{1}$, Adrijana Krsmanovic ${ }^{1}$, Lesley K Carr ${ }^{4}$, Ragi Doggweiler ${ }^{4}$, Robert Moldwin ${ }^{5}$, Robert Mayer ${ }^{6}$

${ }^{1}$ Department of Psychology; ${ }^{2}$ Department of Urology; ${ }^{3}$ Department of Anesthesiology; ${ }^{4}$ Department of Psychology, Queen's University, Kingston; ${ }^{4}$ Sunnybrook Health Sciences Centre, Toronto, Ontario; ${ }^{5}$ Arthur Smith Institute for Urology, New Hyde Park; ${ }^{6}$ University of Rochester Medical Centre, Rochester, New York, USA

AIM: Interstitial Cystitis/Bladder Pain Syndrome (IC/BPS) is a prevalent chronic pelvic pain syndrome whose symptoms include urogenital pain, 
urgency, and frequency (Nickel, 2002). Patients with IC/BPS report lower rates of mental quality of life (QoL) than matched controls (Nickel et al., 2010). The present study aimed to examine the predictors of mental QoL within a biopsychosocial model.

METHODS: 113 women diagnosed with IC/BPS were recruited from tertiary care urology clinics and completed questionnaires (demographics, Medical Outcomes Healthy Survey, McGill Pain Questionnaire, O'Leary Sant, Pain Catastrophizing Scale, Multidimensional Scale of Perceived Social Support, Marital Adjustment Test). A hierarchical multivariate regression analysis was run in the prediction of mental QoL. Step 1 included age and length of marriage; step 2 included pain, IC symptoms, and IC problems; step 3 included catastrophizing, social support, and marital satisfaction.

RESULTS: In step 1 of the regression, age $(\beta=0.378, P=0.007)$ was a significant predictor of mental $\mathrm{QoL}(\mathrm{F}=10.53, \mathrm{P}<0.001)$. In step 2, pain $(\beta=-0.401, P=0.002)$ was a significant predictor of mental $\mathrm{QoL}(\mathrm{F}=9.90$, $\mathrm{P}<0.001)$. In step 3 , social support $(\beta=0.289, \mathrm{P}=0.005)$ and catastrophizing $(\beta=-0.337, P=0.003)$ were predictors of mental $\mathrm{QoL}(\mathrm{F}=10.57, \mathrm{P}<0.001)$, over and above age and pain. Subsequent sub-analyses revealed friends to be the largest contributing source of social support $(\beta=0.192, P=0.083)$ in predicting mental QoL.

CONCLUSIONS: Results show that social support and catastrophizing were significant in predicting mental QoL, over and above the effects of age and pain. Strengthening social support systems and targeting catastrophizing tendencies through psychotherapy interventions may improve IC/ BPS mental QoL.

\section{P116 \\ PAIN AND ANXIETY IN RURAL ACUTE CORONARY SYNDROME PATIENTS AWAITING TRANSFER FOR DIAGNOSTIC CARDIAC CATHETERIZATION}

Sheila O'Keefe-McCarthy' ${ }^{1}$, Michael McGillion ${ }^{2}$, Sioban Nelson ${ }^{3}$, Sean Clarke ${ }^{4}$, Judith McFetridge-Durdle ${ }^{5}$

${ }^{1}$ Lawrence S Bloomberg, Faculty of Nursing, University of Toronto, Toronto, Ross Memorial Hospital, Lindsay, Ontario; ${ }^{2}$ School of Nursing, Faculty of Health Sciences, McMaster University, Hamilton; ${ }^{3}$ University of Toronto, Toronto, Ontario; ${ }^{4}$ The Ingram School of Nursing, McGill University, Montreal, Quebec; ${ }^{5}$ Florida State University, College of Nursing, Tallahassee, Florida, USA

BACKGROUND: In rural areas people suffering from acute coronary syndromes (ACS) wait up to $32 \mathrm{~h}$ for transfer for diagnostic cardiac catheterization (CATH). In lieu of rapid access to CATH, it is critical that pain and anxiety management be optimal in order to preserve myocardial muscle and minimize the risk of further deterioration.

AIM: We examined the relationship between pain management, cardiac pain intensity and state anxiety for rural ACS patients awaiting diagnostic CATH.

METHODS: A prospective, descriptive-correlational repeated-measure design was used to examine the association of analgesic and nitroglycerin administration with cardiac pain intensity (numeric rating scale-NRS) and state anxiety (Speilberger State Anxiety Inventory-SAI) and nurses' pain knowledge and attitudes (Toronto Pain Management Inventory-ACS Version [TPMI-ACS]; Knowledge and Attitudes Regarding Pain Survey [KASP]) via multiple variable, mixed model regression.

RESULTS: The mean age of ACS patients $(n=121)$ was $67.6 \pm 13,50 \%$ were male, $60 \%$ had unstable angina and $40 \%$ had Non-ST-Elevated myocardial infarction. During follow up, cardiac pain intensity scores remained in the mild range from $1.1 \pm 2.2$ to $2.4 \pm 2.7$. State anxiety ranged from $44.0 \pm 7.2$ to $46.2 \pm 6$.6. Cumulative analgesic dose was associated with a reduction in cardiac pain by 1.0 points (NRS, $0-10 ; \mathrm{t}(108)=-2.5$, $\mathrm{SE}=-0.25$, CI $[-0.45,-0.06] ; \mathrm{P}=0.013)$. Analgesic administration was not associated with state anxiety. Throughout the course of follow up, ACS patients reported high anxiety scores indicating a persistent anxious state. CONCLUSIONS: A larger prognostic study is required to determine whether high levels of anxiety for rural patients are predictive of major adverse cardiac events. In the absence of guidelines that address painrelated anxiety assessment and management, development and testing of protocols for effective anxiety management are needed. More urgently, the development and examination of a treatment intervention, early on in the ACS trajectory is warranted that targets pain and anxiety for those who continue to suffer from cardiac pain and persistent high levels of anxiety.

\section{P117}

IMPACT OF PRODROMAL SYMPTOMS ON FUTURE
ADVERSE CARDIAC EVENTS: A SYSTEMATIC REVIEW

Sheila O'Keefe-McCarthy ${ }^{1,2}$, Linda Ready ${ }^{3,4}$

${ }^{1}$ University of Toronto, Toronto; ${ }^{2}$ Ross Memorial Hospital, Lindsay, Ontario; ${ }^{3}$ Athabasca University, Athabasca, Alberta; ${ }^{4}$ City of Kawartha Lakes Family Health Team, Kawartha Lakes, Ontario BACKGROUND: Early recognition of non-specific cardiac prodromal symptoms (CPS), indicative of ischemic cardiac pain, is critical for preemptive coronary artery disease (CAD) screening, effective diagnosis and treatment.

AIM: This systematic review examined whether cardiac prodromal symptoms were predictive of acute painful symptom presentation, cardiac event or intervention.

METHODS: We conducted a systematic review of English language research articles on prodromal symptoms published between1990 and 2013.

RESULTS: Seven studies examined 6716 individuals with CPS $(65.7 \%$ women). Mean average age was $68 \pm 13$ and $58.5 \pm 9$ years for women and men, respectively. Cardiac prodromal symptoms were predictive of patients' cardiac pain and associated adverse events from three to 24 months. Prodromal symptoms consistently reported prior to cardiac event were: arm pain/discomfort, jaw pain, back/shoulder blade pain, fatigue, shortness of breath $(\mathrm{SOB})$, and sleep disturbance. Patients with prodromal arm, jaw, back pain, fatigue and $\mathrm{SOB}$ had increased risk of experiencing similar symptoms during an ACS episode. For men, prodromal symptoms were predictive of effective transfer for cardiac intervention but not for women. There is some preliminary evidence to suggest that prodromal symptoms of headache, sleep disturbance and anxiety may predict acute coronary syndrome pain during an acute cardiac event.

CONCLUSIONS: Future research is warranted that would examine the predictive value of prodromal headache, sleep disturbance and anxiety within this cardiovascular population. Pre-emptive screening for cardiacrelated prodromal symptoms in men and women should be considered as a standard in clinical practice. This would potentiate early diagnosis, effective risk modification, timely pain management and decrease CAD-related morbidity and mortality.

\section{P118}

\section{PAIN PROCESSING, EMPATHY AND CATASTROPHIZING IN PEOPLE WITH MULTIPLE SCLEROSIS FOLLOWING ACUTE PAIN INDUCTION}

Camille Olechowski ${ }^{1}$, Michelle Verrier $^{1}$, Fabrizio Giuliani ${ }^{2}$, Bruce Dick ${ }^{1,2}$

${ }^{1}$ Department of Anesthesiology and Pain Medicine; ${ }^{2}$ Centre for Neuroscience, University of Alberta, Edmonton, Alberta

AIM: While pain has long been known to be a common symptom among Multiple Sclerosis (MS) patients, it has received shockingly little attention in the research literature. MS pain affects as many as one in five patients at disease onset, and as many as half of patients at any given point in their disease. The aims of this study were to determine how MS patients with and without chronic pain process acute pain.

METHODS: MS patients and matched healthy controls watched a video of an actor undergoing the cold pressor test (CPT) and were randomized either to watching a video where the actor demonstrated pain behaviour reflective of a catastrophic response to CPT or another video where the actor showed a stoic response. Participants then underwent CPT for $2 \mathrm{~min}$ with pain ratings recorded every $30 \mathrm{~s}$.

RESULTS: MS patients demonstrated increased sensitivity to acute CPT pain. MS patients in the progressive stages of the disease were significantly more sensitive to the CPT test. MS patients with existing chronic pain reported a significant decrease in their primary pain location after completing the CPT, consistent with central inhibitory pain processes (eg, DNIC). 
CONCLUSIONS: Regardless of whether or not MS patients presented with overt pain symptoms, increased sensitivity to a painful cold stimulus was observed. This finding may be suggestive of central alterations in somatosensory processing in this population that are likely critical to study and treat.

\section{P119}

\section{A DISTRACTION TECHNIQUE FOR PAIN RELIEF IN HOSPITALIZED CHILDREN: A RANDOMIZED CONTROLLED STUDY}

Nátali Castro Antunes Caprini Oliveira, Maria Beatriz Martins Linhares Ribeirão Preto Medical School, University of São Paulo, São Paulo,

\section{Brazil}

AIM: To evaluate the effectiveness of an intervention of distraction for pain relief in hospitalized children undergoing acute painful procedures. METHODS: Design study: randomized control cross-over study. Methods: the sample was 40 children at school-age (six to 12 years) admitted in a Pediatric ward, who undergoing venipuncture procedures. The sample was randomized into two groups, Distraction group (DG) and Control group (CG). In the intervention, the psychologist offered to children audiovisual distraction (short films) before and during the painful procedure. The pain assessment after the procedure used the child's reports in the Faces Scale Revised (FPS-R) and the Visual Analogic Scale (VAS).

RESULTS: There were statistically significant differences between groups in both pain scales; the IDG presented lower pain scores in FPS-R (mean, $\mathrm{DG}=1.91 \pm 1.68$ and $\mathrm{CG}=6.78 \pm 3.15 ; \mathrm{P} \leq 0.05$ ) and VAS (mean, $\mathrm{DG}=1.50 \pm 1.87$ and $\mathrm{CG}=6.28 \pm 2.86 ; \mathrm{P} \leq 0.05$ ), in comparison to $\mathrm{CG}$.

CONCLUSIONS: The intervention of distraction reduced the pain perception during routine acute-pain procedure in hospitalized children.

\section{P120 \\ NON-PHARMACOLOGICAL INTERVENTIONS FOR PAIN RELIEF IN CHILDREN: A SYSTEMATIC REVIEW}

Nátali Castro Antunes Caprini Oliveira, Maria Beatriz Martins Linhares Ribeirão Preto Medical School, University of São Paulo, São Paulo, Brazil

AIM: The present study systematically reviewed the updated literature that has been published in the last five years (2008 to 2013) regarding the effectiveness of non-pharmacological management for acute pain relief in sick children of preschool and school age.

METHODS: The literature review was performed by selecting scientific articles indexed in main databases. The keywords used in the search were the following: pain and non-pharmacological and management; pain and non-pharmacological and intervention; acute and procedural and pain; pain and intervention and distraction.

RESULTS: We analyzed 12 empirical articles. Seven were randomized controlled trials, and five were clinical trials with no randomization. The pain outcomes of the studies included validity measures by self-report, hetero-report, and behavioral observation. All 12 studies included at least one intervention that used a distraction strategy. Pain management that used audiovisual distraction, virtual reality, distraction with objects (eg, cards, a kaleidoscope, and a soft ball), distraction performed by parents or professionals, and multimodal distraction device interventions significantly reduced pain scores in children who underwent different painful procedures.

CONCLUSIONS: The findings of the present review suggest that distraction may be recommended as a simple and efficient non-pharmacological acute pain relief strategy to be implemented in clinical practice in pediatric care settings. Future studies should be conducted to assess different medical procedures and settings to better generalize the results.

\section{P121}

INFANT NEGATIVE FACIAL EXPRESSIONS OF PAIN FOLLOWING IMMUNIZATION PROCEDURES OVER THE FIRST YEAR OF LIFE

Monica $\mathrm{O}^{\prime}$ Neill $^{1}$, Sara Ahola Kohut ${ }^{2}$, Rebecca Pillai Riddell1,2, Harriet Oster ${ }^{3}$

${ }^{1}$ York University; ${ }^{2}$ The Hospital for Sick Children, University of Toronto, Toronto, Ontario

AIM: To determine if the average time spent displaying five different negative facial expression categories over $1 \mathrm{~min}$ post-needle differs at two, four, six and 12 months of age.

METHODS: Video footage of infants' facial expressions over 1 min post needle was coded using the Facial Action Coding System for Infants and Young Children (BabyFACS; Oster, 2010). Five negative facial expressions categories were developed according to the most frequently co-occurring negative facial expressions identified.

RESULTS: The average time spent in each of the facial expression categories was calculated for four $15 \mathrm{~s}$ epochs over $1 \mathrm{~min}$ post-needle (ie, $0 \mathrm{~s}$ to $14 \mathrm{~s}, 15 \mathrm{~s}$ to $29 \mathrm{~s}, 30 \mathrm{~s}$ to $44 \mathrm{~s}, 45 \mathrm{~s}$ to $59 \mathrm{~s}$ ) at each immunization appointment (two, four, six and 12 months). A set of factorial repeated measures ANOVAs were performed to compare the amount of time that infants displayed each of the negative facial expression categories within four $15 \mathrm{~s}$ epochs over $1 \mathrm{~min}$ post needle at each immunization appointment. There was a significant interaction between the $15 \mathrm{~s}$ epochs and the mean amount of time that the negative facial expression categories were displayed at two months, $\mathrm{F}(6.18,612.21)=25.19, \mathrm{P}<0.001$, partial $\eta^{2}=0.20$, four months, $F(5.53,547.42)=24.29, P<0.001$, partial $\eta^{2}=0.20$, six months, $\mathrm{F}(6.14,607.72)=16.32, \mathrm{P}<0.001$, partial $\eta^{2}=0.14$, and 12 months of age, $\mathrm{F}(5.71,565.59)=7.84, \mathrm{P}<0.001$, partial $\eta^{2}=0.07$.

CONCLUSIONS: Infant negative facial expressions are displayed for a significantly different amount of time within four $15 \mathrm{~s}$ epochs over $1 \mathrm{~min}$ post needle for each age group.

\section{P122}

\section{SMARTPHONE AND INTERNET PATTERNS OF PARENTS (SIPP): PREFERENCES AND IMPLICATIONS FOR KNOWLEDGE UPTAKE IN THE NICU}

$\underline{\text { Talia Orr }^{1}}{ }^{1}$ Brenda Hewitt ${ }^{1}$, Jennifer Stinson ${ }^{2}$, Patrick McGrath ${ }^{1}$, Marsha Campbell-Yeo ${ }^{1}$

1'IWK Health Centre and Dalhousie University, Halifax, Nova Scotia; ${ }^{2}$ The Hospital for Sick Children, Toronto, Ontario

AIM: To understand the patterns of smartphone and Internet use by parents in the NICU, to learn more about parent information seeking behaviours, and to discover new strategies to increase parent involvement in infant pain management

METHODS: A descriptive study using a paper-based survey distributed to parents in a tertiary level NICU from September-November, 2013.

RESULTS: Response rate was $80 \%$ (72 of 90 ). Almost all parents reported that they would like to have more information about how they can provide general comfort (96\%), as well as how to be more actively involved in comforting their baby during painful procedures (94\%). The majority of participants $(n=70[97 \%])$ access the Internet on a daily basis, $87 \%$ of those access using their smartphone (100\% of fathers, $82 \%$ of mothers). The Internet was ranked as an important source of NICU information by $81 \%$ of parents, followed by books $(56 \%)$ and brochures $(33 \%)$. NICU nurses (96\%), NICU doctors (89\%) and family doctors (82\%) were also ranked as important information resources. Of those who researched helping their baby cope with pain, $56 \%$ used the Internet as their primary source.

CONCLUSIONS: Parents report a desire to have more information and be more involved in infant pain management. Parents in the NICU place higher value on the Internet compared to books or brochures, which are the education tools most commonly used in the IWK. It is imperative that health facilities take advantage of the Internet as an accessible tool to enhance health professional teaching and increase parent involvement 
P123

ACUTE PAIN TRAJECTORIES AND THE DEVELOPMENT OF CHRONIC POST-SURGICAL PAIN: A LONGITUDINAL STUDY OF TOTAL HIP ARTHROPLASTY

M Gabrielle Pagé ${ }^{1}$, Joel Katz ${ }^{1,2,3}$, Kathryn Curtis ${ }^{1}$, Noga Cohen ${ }^{1}$, E Manolo Romero Escobar ${ }^{1}$, Hance Clarke ${ }^{2,3}$

${ }^{1}$ Department of Psychology, Faculty of Health, York University;

${ }^{2}$ Department of Anesthesia and Pain Management, Toronto General Hospital; ${ }^{3}$ Department of Anesthesia, Faculty of Medicine,

University of Toronto, Toronto, Ontario

AIM: The aim of this study was to explore acute post-operative pain intensity trajectories over the first four days after surgery and examine how these trajectories are associated with the presence of chronic pain and pain disability up to six months after total hip arthroplasty (THA).

METHODS: One hundred forty-seven adults ( $\mathrm{n}=80$ female [49.4\%]; mean [ $\pm \mathrm{SD}$ ] age $60.2 \pm 9.1$ years) completed the 11-point Numerical Rating Scale for pain intensity and other pain-related questionnaires preoperatively, several times daily until hospital discharge, daily over the first week post-hospital discharge as well as six weeks and six months after surgery.

RESULTS: Using Growth Mixture Model, the best fitting model (AIC = 8808.37) had four acute post-operative pain intensity trajectories (Trajectory 1: initially low/moderate pain with a steady decline over time; Trajectory 2: initially low/moderate pain that remains constant over time; Trajectory 3: initially moderate/high pain with a rapid and steady decline over time; Trajectory 4 : initially moderate/high pain with a slow and steady decline over time). Multivariate ANOVA showed that individuals in Trajectory 2 six weeks post-operatively and in Trajectory 4, six months post-operatively had higher levels of pain disability compared to individuals in Trajectories 1 and 3 and Trajectories 2 and 3, respectively.

CONCLUSIONS: These results highlight the need to consider the heterogeneity of patients' post-operative pain experience and suggest that pain variations across the first few days after surgery might help identify individuals who are at higher risk for pain disability in the weeks and months after THA.

\section{P124}

NOT ALL POST-OPERATIVE PAIN IS ALIKE: A PAIN TRAJECTORY ANALYSIS IN TOTAL KNEE ARTHROPLASTY PATIENTS

M Gabrielle Pagé ${ }^{1}$, Joel Katz ${ }^{1,2,3}$, Kathryn Curtis ${ }^{1}$, Samantha Fuss ${ }^{1}$, Noga Cohen', E Manolo Romero Escobar ${ }^{1}$, Hance Clarke ${ }^{2,3}$

${ }^{1}$ Department of Psychology, Faculty of Health, York University;

${ }^{2}$ Department of Anesthesia and Pain Management, Toronto General

Hospital; ${ }^{3}$ Department of Anesthesia, Faculty of Medicine,

University of Toronto, Toronto, Ontario

AIM: Examining pain trajectories from acute to chronic post-surgical pain (CPSP) can provide important information about a patient's risk of developing CPSP. The goal of this study was to examine individuals' pain trajectories pre-operatively up to 12 months after surgery.

METHODS: One hundred eighty four participants completed pain and psychological questionnaires and functional performance tests pre-operatively and four days, six weeks, and three and 12 months after total knee arthroplasty (TKA).

RESULTS: Using a Growth Mixture Model, results showed that a fourclass model $(\mathrm{AIC}=2772.27)$ with a quadratic slope and baseline data as a predictor of class membership best fit the pain data (WOMAC pain subscale). Trajectories 1 to 3 represent patients with either low or high preoperative pain but experience a decrease in pain (or maintenance of low pain levels) over the first few days post-operatively. These individuals also exhibit lower levels of pain 12 months after surgery. In contrast, individuals in Trajectory 4 experience moderate pre-operative pain that remains constant over the first few days after surgery. These individuals do not recover from their pain and instead continue to experience moderate pain 12 months after surgery.

CONCLUSIONS: This model suggests that moderate pre-operative pain, as opposed to low or high pain, is a risk factor for a neutral/positive pain trajectory post-operatively. Consistent with previous studies, these results show that post-operative pain is heterogeneous and point to the importance of examining fluctuations in pain levels as opposed to static pain scores (eg, preoperative pain score) in assessing one's risk of developing CPSP.

\section{P125}

\section{A NEW OBSERVATION SCALE FOR ASSESSING PATIENT-} CENTERED CARE IN CHRONIC PAIN PATIENTS

Emilie Paul-Savoie $^{1}$, Sylvie Lafrenaye ${ }^{2}$, Stéphane Potvin ${ }^{3}$, Patricia Bourgault ${ }^{1}$

${ }^{1}$ School of Nursing, Faculty of Medicine and Health Sciences; ${ }^{2}$ Department of Pediatrics, Faculty of Medicine and Health Sciences, Université de Sherbrooke; ${ }^{3}$ Centre de recherche de I'Institut Universitaire en Santé Mentale de Montréal, Department of Psychiatry, Faculty of Medicine, Université de Montréal, Montreal, Quebec

AIM: Patient-centered care (PCC) has been associated with good clinical outcomes for chronic pain patients. Various methods have been used for measuring PCC in healthcare providers, such as self-rating and observer rating. Self-assessment instruments are more subjective and susceptible to social desirability bias. Observation instruments are more objective and can be used in clinical situations. Few observation instruments exist to measure PCC, but there has been no scale specifically designed to assess PCC in the context of chronic pain using real patients videos. The aim of this study was to develop and validate an observation scale (OS) for the assessment of PCC in physicians and nurses, using standardized videos of chronic pain patients.

METHODS: A 11-items preliminary French version of the OS was developed based on a review of the literature. After a revision by a group of seven experts in the healthcare field of pain, a revised nine-item version of the instrument was tested in a sample of 21 physicians and 21 nurses. These participants watched five videos of real patients with chronic pain. Participants had to explain the treatment plan that they would provide. Three independent observers evaluated the use of PCC demonstrated by the participants using the OS.

RESULTS: The expert panel was satisfied with the content validity of the scale. The results showed good inter-rater reliability.

CONCLUSIONS: Psychometric findings provided support for the content and the inter-rater reliability. The OS has good potential for future research in French populations and will be validated in English.

\section{P126 \\ LINKING CUSTOMIZED KNOWLEDGE TRANSLATION INITIATIVES TO PEDIATRIC PAIN OUTCOMES}

Jennifer Peleshok, Janet Yamada, Anne-Marie Krancevic, Carol McNair, Munira Nanji, Andrea LeBlanc, Jacqueline Hanley, Bonnie Stevens, Fiona Campbell

University of Toronto, The Hospital for Sick Children, Toronto, Ontario

AIM: To determine the effect of an inpatient hospital unit pain resource group (PRG) at a single Canadian tertiary care pediatric hospital on pediatric pain process and clinical outcomes.

METHODS: Three units developed different models of PRGs for customizing knowledge translation initiatives to raise awareness, provide education and deliver feedback to nursing staff. Unit A developed a new PRG (established Q2; 2013/2014). Unit B relied on unit leaders and a pain champion. Unit C utilized their Pain Committee (establised 2005). Clinical and process outcomes were measured using a $24 \mathrm{~h}$ snap-shot audit tool administered quarterly.

RESULTS: Unit A: Pain assessments were completed on $92 \%$ of patients (Q1) and increased to $100 \%$ (Q2). At Q1, 60\% of patients with moderate to severe pain had no interventions; this decreased to $0 \%$ (Q2/Q3). The prevalence of moderate to severe pain fluctuated between 20\% (Q1) and 16\% (Q3). Unit B: Pain assessments were completed on $100 \%$ of patients. The number of patients with interventions following assessment of moderate to severe pain remained stable at $86 \%(\mathrm{Q} 1)$ to $87 \%(\mathrm{Q} 3)$. The prevalence of moderate to severe pain fluctuated over time (39\% Q1 to 36\% Q3). 
Unit C: Pain assessments were done on $100 \%$ of patients. All patients with moderate to severe pain received an intervention. Prevalence of moderate to severe pain fluctuated: $12 \%(\mathrm{Q} 1)$ to $16 \%(\mathrm{Q} 2 / \mathrm{Q} 3)$.

CONCLUSIONS: The implementation and duration of a dedicated pain resource group positively affects pain process outcomes. However, clinical outcomes appear to be independent of the model of resource group.

\section{P127}

\section{WITHDRAWN}

\section{P128}

DEVELOPMENT OF CHILD ANTICIPATORY DISTRESS TO PAINFUL MEDICAL PROCEDURES: A SYSTEMATIC REVIEW Nicole Racine ${ }^{1}$, Rebecca Pillai Riddell ${ }^{1,2}$, Anna Taddio ${ }^{2}$, Elizabeth Uleryk ${ }^{2}$

${ }^{1}$ York University; ${ }^{2}$ The Hospital for Sick Children, University of Toronto, Toronto, Ontario

AIM: Unmanaged distress during painful medical procedures have been associated with fear of medical procedures and avoidance of preventative medical care (Wright et al., 2009). Child anticipatory anxiety is associated with higher pain-related distress after a painful medical procedure (Bijttebier \& Vertommen, 1998). Little empirical work has investigated the predictors of child anticipatory distress to pain. The aim of the current study is to conduct a systematic review of the factors that predict anticipatory distress to painful medical procedures in children.

METHODS: A systematic search was conducted in MEDLINE, EMBASE, and EBM Reviews - Cochrane Central Register of Controlled Trials (CCTR) and PsycINFO to include articles indexed as of November 20, 2013. Additional hand searches were also performed. The search strategy retrieved a total of 8936 references. A total of 1875 duplicates were identified and the remaining 7061 articles were reviewed against in the inclusion criteria. Search results were limited to evidence-based study design methodologies, publication years $(2001+)$ and age group (children zero to 18 years of age). Studies were screened for eligibility and reliability was achieved among reviewers.

RESULTS: Studies were identified as falling under three main domains: child-related factors, parent-related factors, and contextual-related factors. Child genetics and temperament, anxious parental disposition, parent behaviour, child threat appraisal, and experience during previous painful events are all hypothesized to contribute to the development of anticipatory distress in children.

CONCLUSIONS: Longitudinal research is needed to prospectively investigate the factors that contribute to the development of anticipatory distress to painful medical procedures in children.

\section{P129 \\ DISABILITY IN FIBROMYALGIA IS ASSOCIATED WITH GREATER SELF-REPORTED SYMPTOMS AND FUNCTIONAL IMPAIRMENT}

E Rampakakis ${ }^{1}$, Mary-Ann Fitzcharles ${ }^{2}$, PA Ste-Marie ${ }^{3}$, JS Sampalis ${ }^{4}$, Y Shir $^{2}$

${ }^{1}$ JSS Medical Research; ${ }^{2}$ Montreal General Hospital; ${ }^{3}$ University of Montreal; ${ }^{4}$ Jewish General Hospital, Montreal, Quebec

AIM: Disablement due to illness should reflect illness severity. When illness measurement is based on subjective report the reliability of report is crucial. We have examined clinical characteristics of FM patients currently employed or receiving disability payments.

METHODS: Of $246 \mathrm{FM}$ patients in a cohort study, 77 were receiving disability payments (Group 1), 90 were employed (Group 2). Demographic and disease severity measures were compared for the two groups.

RESULTS: The prevalence of disablement was 30.8\%. Differences for Group 1 versus Group 2 were examined respectively: age, 49.1 years versus 45.9 years $(\mathrm{P}=0.020)$; smoking, $33.8 \%$ versus $15.6 \%(\mathrm{P}=0.006)$; marijuana use, $13.0 \%$ versus $3.3 \%(\mathrm{P}=0.020)$. Pain duration (10.7 years) and gender (female: $91.0 \%$ ) did not differ. Prior/current occupation differed significantly between groups: Group 1 manual professions or service industry, Group 2 education/clerical/health fields $(\mathrm{P}=0.001)$. Management strategies differed as follows: Group 1 greater count medications $(P=0.001)$, opioids $(\mathrm{P}=0.001)$, antidepressants $(\mathrm{P}=0.032)$, tranquilizers $(\mathrm{P}<0.001)$, and cannabinoids $(\mathrm{P}=0.053)$, and exercised less $(\mathrm{P}=0.009)$. Group 1 had more allodynia $(\mathrm{P}=0.027)$ and pain related behaviour $(\mathrm{P}=0.002)$. Except for depression and anxiety, all other disease-related parameters were significantly higher in Group 1.

CONCLUSIONS: A significant proportion of FM patients are unemployed due to disability. The subjective more severe symptom report may be explained by true disease severity, negative impact of medications, or patient perception of illness. Alternately, justification for ongoing disablement may drive augmentation of subjective illness report. As all measurements in FM are subjective, disabled patients may be an important confounder for understanding outcome in FM.

\section{P130}

\section{WITHDRAWN}

\section{P131}

\section{RELATIONSHIP BETWEEN PATIENT SATISFACTION AND TREATMENT OUTCOMES IN CHRONIC PAIN COMMUNITY CLINICS}

\section{Kim Rod}

McMaster University, Hamilton, Ontario

AIM: Chronic pain remains a major health crisis. In Canada alone, one in three persons (33\%) lives with chronic pain. Multidisciplinary treatments for chronic pain are shown to be effective. The objective of this case study was to determine whether patient satisfaction affects treatment outcomes. METHODS: Patients enrolled in an eight-week multidisciplinary pain program in a community pain clinic. Patients completed questionnaires about pain, psychological and emotional status, and satisfaction with program. Select questions were asked before and after the program and were designed to include patients' evaluation of the program, level of change, pain and coping perceptions, and psychological/emotional adjustment. Scales ranged according to the question.

RESULTS: Patients $(\mathrm{n}=62)$ completed questionnaires. Over half $(52.54 \%)$ rated their overall level of satisfaction with the treatment program as very helpful. At the start of the program, $24 \%$ of patients rated their level of pain as severe compared to $1.67 \%$ at the end of the treatment. All of the patients who completed the questionnaires reported that they would recommend the program to friends.

CONCLUSIONS: The results of this survey suggest a relationship between patient satisfaction and treatment outcome. More than half $(52.54 \%)$ of patients felt the treatment program was very helpful, and all the patients reported that they would recommend the program to friends. The effect of patient satisfaction on treatment outcomes warrants further investigation by well organized studies.

\section{P132}

\section{EVALUATION OF AN AUTOMATED PARENT-TRAINING PROGRAM FOR PARENTS' BEHAVIOR DURING CHILDREN'S IMMUNIZATIONS}

Nikita Rodrigues ${ }^{1}$, Lindsey L Cohen ${ }^{1}$, Donald J Bearden ${ }^{1}$, Naomi E Joffe ${ }^{2}$, Josie S Welkom ${ }^{3}$, Patrick J McGrath ${ }^{4}$

${ }^{1}$ Georgia State University, Atlanta, Georgia; ${ }^{2}$ Department of Pediatrics, University of Cincinatti, Cincinatti, Ohio; ${ }^{3}$ University of Maryland, Baltimore County, Maryland, USA; ${ }^{4}$ Dalhousie University, Halifax, Nova Scotia

AIM: The majority of children find immunization injections distressing. The literature indicates that parent behavior accounts for a large proportion of the variance in child distress. We evaluated a brief automated computer program for teaching parents to engage in evidence-based behavior during their child's immunizations.

METHODS: 80 four- to six-year-olds (42 female) receiving immunizations and their parents participated. Twenty-seven were assigned to Control, 25 to Distraction Only, and 28 to Distraction Plus Training. Videotapes of parent behaviors were coded for occurrence using an adapted Child-Adult Medical Procedure Interaction Scale (CAMPIS; Blount et al., 1989). 
RESULTS: 3 (condition) $\times 3$ (phase) ANOVAs were conducted and main effects for phase were found for humor $(\mathrm{P}=0.04)$, reassurance $(\mathrm{P}<0.001)$, providing information $(\mathrm{P}=0.011)$, and praise $(\mathrm{P}<0.001)$. A main effect for condition was found for encouraging deep breathing, $\mathrm{F}(2,77)=5.86, \mathrm{P}=0.004, \mathrm{r}=0.36$. Contrasts indicated that parents in the Training Plus Distraction condition encouraged deep breathing significantly more than those in the Control condition $(\mathrm{P}=0.011)$ and than those in the Distraction Only condition $(\mathrm{P}=0.004)$. A significant main effect for condition was found for distraction, $\mathrm{F}(2,77)=4.77, \mathrm{P}<0.001$, $r=0.33$, with parents in Training Plus Distraction using distraction significantly more than those in Control $(\mathrm{P}=0.003)$ or Distraction Only $(\mathrm{P}=0.019)$ conditions.

CONCLUSIONS: Findings indicate that although the Distraction Plus Training computer program did not significantly increase all targeted behaviors (praise, humor, play), it was effective in increasing parents' use of deep breathing and distraction strategies.

\section{P133}

PAIN AND SYMPTOMS OF POST-TRAUMATIC STRESS IMMEDIATELY FOLLOWING TRAUMATIC MUSCULOSKELETAL INJURY: A MODIFIED DIATHESISSTRESS MODEL OF PSYCHOLOGICAL DISTRESS

Brittany N Rosenbloom ${ }^{1,2}$, Colin JL McCartney ${ }^{1,2}$, Kelly Chin², Lynn Haslam ${ }^{1}$, Richard Foty ${ }^{1}$, Farida Roy ${ }^{2}$, Hans Kreder ${ }^{1,2}$, Joel Katz ${ }^{1,3}$

${ }^{1}$ University of Toronto; ${ }^{2}$ Sunnybrook Health Sciences Centre; ${ }^{3}$ York University, Toronto, Ontario

AIM: Traumatic musculoskeletal injury often leads to chronic pain and post-traumatic stress disorder (PTSD). Chronic post-traumatic/ post-surgical pain and PTSD may be the product of a modified diathesis-stress model including shared vulnerabilities and mutually maintaining factors. The aim of the current study was to examine the relationships between factors involved in the modified model on symptoms of PTSD during the acute period following injury.

METHODS: 205 patients $(67.80 \%$ male, mean $[ \pm$ SD] age $43.02 \pm 19.90$ years, mean [ \pm SD] Injury Severity Score of $16.57 \pm 8.97$ ) were recruited to participate in a prospective, observational, cross-sectional design study employed to investigate acute symptoms of anxiety, depression, PTSD, and pain, as well as anxiety sensitivity, fear of pain, pain catastrophizing, and self-efficacy with pain. Participants completed a questionnaire in-hospital within 14 days of their injury. Data was collected from patient charts on injuries and analgesic consumption.

RESULTS: The logistic regression analysis used to determine the impact of multiple predictor factors on the presence of PTSD posttraumatic on severe symptoms of PSTD was significant $(\mathrm{P}<0.0001)$. Specifically, neuropathic pain (odds ratio $[\mathrm{OR}]=1.092 ; \mathrm{P}=0.011)$, general anxiety $(\mathrm{OR}=1.176$; $\mathrm{P}=0.006)$, pain anxiety $(\mathrm{OR}=1.056 ; \mathrm{P}=0.003)$, and pain catastrophizing $(\mathrm{OR}=1.168 ; \mathrm{P}=0.032)$ uniquely predicted participants with severe symptoms of PTSD.

CONCLUSIONS: The results support the previously proposed modified diathesis-stress model indicating that neuropathic pain, general anxiety, pain anxiety, and pain catastrophizing are significantly associated with severe symptoms of PTSD. Future studies should prospectively examine the influence of these acute factors on the development of chronic pain and posttraumatic stress disorder following injury.

\section{P134}

\section{SEASONAL VARIATIONS IN PEDIATRIC COLD PRESSOR} PAIN

Meghan G Schinkel ${ }^{1,2}$, Kristen S Higgins ${ }^{1,2}$, Hannah G Gennis ${ }^{1,2}$, Jennifer A Parker ${ }^{2}$, Christine T Chambers ${ }^{1,2}$

${ }^{1}$ Departments of Psychology and Neuroscience, Dalhousie University; ${ }^{2}$ Centre for Pediatric Pain Research, IWK Health Centre, Halifax, Nova Scotia

AIM: There is evidence to suggest that factors such as weather and seasonal variations may influence pain (eg, Jones et al., 2005; Macfarlane et al., 2010). The cold pressor task (CPT) is a safe and ethically appropriate task that induces mild to moderate pain (von Baeyer et al., 2005), and is widely used to experimentally investigate pain in children. The current study aimed to examine whether seasonal variations exist in child responses to cold pressor pain.

METHODS: Data was pooled from 155 children (boys, $\mathrm{n}=81$; girls, $\mathrm{n}=74$ ) between eight and 14 years of age (mean 9.64 years of age) who had participated in the cold pressor task as part of one of four previous studies. Child pain tolerance (time in seconds the child left their hand immersed in the cold water) and child reported pain intensity (Faces Pain Scale-Revised rating) were recorded for each participant following the CPT. Participation occurred across all four seasons: winter $(n=21)$, spring $(n=38)$, summer $(\mathrm{n}=66)$ and fall $(\mathrm{n}=30)$.

RESULTS: Two separate one-way ANOVAs were conducted to examine the influence of season on pain intensity and pain tolerance. The analyses revealed no significant differences for season for both child pain intensity and child pain tolerance.

CONCLUSIONS: There appears to be no influence of season on both child pain intensity and pain tolerance for cold pressor pain.

\section{P135}

PERCEIVED SOURCES OF INJUSTICE IN PATIENTS WITH PERSISTENT PAIN FOLLOWING ACCIDENTAL INJURY

Whitney Scott ${ }^{1}$, Maria Milioto ${ }^{2}$, Amanda McEvoy ${ }^{1}$,

Rosalind Garland ${ }^{1}$, Zina Trost ${ }^{3}$, Elena Bernier ${ }^{1}$, Michael JL Sullivan ${ }^{1}$

${ }^{1}$ McGill University; ${ }^{2}$ Centre D'Evaluation Et De Réadaptation De L'Est, Montreal, Quebec; ${ }^{3}$ University of North Texas, Denton, Texas, USA

AIM: Perceived injustice is emerging as an important psychosocial risk factor for problematic recovery following painful injury. To date, limited research has investigated sources of perceived injustice among patients with persistent pain following injury. Therefore, the purpose of this study was to identify sources of perceived injustice in patients with persistent pain after injury.

METHODS: The sample consisted of 90 patients with persistent musculoskeletal pain following a whiplash or occupational injury. Participants completed the Injustice Experiences Questionnaire and a semi-structured interview in which they reflected on sources of unfairness and blame in the context of their injury and persistent pain. Interview responses were coded by two independent raters to identify common sources of perceived injustice.

RESULTS: Coding revealed a number of sources of perceived injustice, including other drivers, employers, insurers, and medical professionals. The identification of these sources varied, in part, as a function of the type of injury (ie, whiplash versus occupational low back). Reasons underlying injustice perceptions differed in part according to the identified source. CONCLUSIONS: A number of sources may contribute to perceptions of injustice among individuals with persistent pain following injury. Implications of the findings for individual-and systems-level interventions will be discussed.

\section{P136}

\section{PSYCHOMETRIC PROPERTIES OF THE PAIN BELIEFS} QUESTIONNAIRE FOR CHILDREN AND ADOLESCENTS

Amanda L Sherman ${ }^{1}$, Craig A Smith ${ }^{1}$, Kelsey T Laird ${ }^{1}$, Kezia C Shirkey ${ }^{2}$, Lynn S Walker ${ }^{3}$

${ }^{1}$ Department of Psychology and Human Development, Vanderbilt University, Nashville, Tennessee; ${ }^{2}$ North Park University, Chicago, Illinois; ${ }^{3}$ Department of Pediatrics, Vanderbilt University School of Medicine, Nashville, Tennessee, USA

AIM: Beliefs about pain are a central component of pain perception and response to pain. The importance of pain beliefs has emerged in the pediatric literature in several studies using the Pain Beliefs Questionnaire (PBQ), a self-report measure developed to assess pain related beliefs of children and adolescents. This study evaluates the psychometric properties of the PBQ (ie, internal consistency, test-retest reliability, and construct validity) in youth with functional abdominal pain (FAP).

METHODS: The sample included 871 consecutive new patients (mean age: $11.56 ; 62 \%$ female) referred to a pediatric gastroenterology clinic for evaluation of FAP. Patients completed questionnaire measures (ie, PBQ 
and measures of pain, pain coping, self-efficacy, and disability) in the clinic and a subsample completed follow-up questionnaires two weeks $(n=305)$ and six months $(\mathrm{n}=123)$ later.

RESULTS: Three conceptually derived subscales (Pain Threat Appraisal [PTA], Problem Focused Coping Potential [PFCP], Emotion Focused Coping Potential [EFCP]) demonstrated high levels of internal consistency (PTA: $\alpha=0.80$; PFCP: $\alpha=0.79$; EFCP: $\alpha=0.79$ ). Subscales exhibited adequate test-retest reliability at two weeks (PTA: $r=0.75$; PFCP: $r=0.75$; EFCP: $r=0.77$ ) and six months (PTA: $r=0.47$; PFCP: $r=0.51$; EFCP: $\mathrm{r}=0.53$ ). Subscales exhibited construct validity through analyses examining their interrelation and relation to measures of pain coping, self-efficacy, pain severity, and disability.

CONCLUSIONS: Results established the PBQ as a valid and reliable measure for assessing children and adolescent's beliefs about pain and their ability to cope with pain.

\section{P137}

COGNITIVE FUSION QUESTIONNAIRE FOR ADOLESCENTS (CFQ-A): A PRELIMINARY STUDY OF ITS PSYCHOMETRIC PROPERTIES

\section{Ester Solé, Jordi Miró}

Unit for the Study and Treatment of Pain ALGOS; Research Center for Behavior Assessment, Psychology Department, Institut d'Investigació Sanitària Pere Virgili Universitat Rovira i Virgili, Catalonia, Spain

AIM: Cognitive Fusion, that is, "the tendency for behavior to be overly regulated and influenced by cognition"1 (p.2) is a concept from Acceptance and Commitment Therapy which is becoming of central interest in the management of chronic pain. The main objectives of this work were to (1) adapt the Cognitive Fusion Questionnaire to be used with Catalanspeaking adolescents, and (2) assess its psychometric properties when it is self-administered, namely: construct validity, by examining its factorial structure, and validity against some criteria such as anxiety sensitivity, pain catastrophizing and mindfulness.

METHODS: A total of 344 adolescents (11 to 20 years of age) participated in this study. Fifty-nine percent were girls with a mean $( \pm$ SD) age of $14.36 \pm 1.66$ years. Participants reported on the pain intensity they had experienced during the three months before the interview, and completed questionnaires measuring anxiety sensitivity, pain catastrophizing, mindfulness, disability, and pain coping strategies.

RESULTS: Factorial analyses revealed a one-factor solution $\left(\chi^{2}=29.63\right.$, $\mathrm{P}<0.01 ; \mathrm{CFI}=0.97$; RMSEA=0.06). Cronbach's Alfa was 0.78. Criteria validity was supported by significant positive correlations between scores on the CFQ-A and criteria variables: anxiety sensitivity, catastrophizing, mindfulness, maximum intensity of the most frequent pain, disability and some coping strategies (eg, externalizing), as well, by significant negative correlations with behavioral and cognitive distraction strategies.

CONCLUSIONS: The CFQ-A has an acceptable level of validity and reliability. The one-factor solution suggested for adults has been confirmed in our sample of schoolchildren.

Footnotes/References:

1. Gillanders DT, Bolderston H, Bond FW, et al. The Development and Initial Validation of the Cognitive Fusion Questionnaire. Behav Ther 2013; http://dx.doi.org/10.1016/j.beth.2013.09.001

\section{P138 \\ COMPARISON OF CANADIAN AND ISRAELI RHEUMATOLOGISTS' UNDERSTANDING OF CANNABINOID USE AS A THERAPY FOR RHEUMATIC DISEASES}

Peter Ste-Marie $^{1}$, Yoram Shir ${ }^{1}$, Jacob Ablin ${ }^{2}$, Mary-Ann Fitzcharles ${ }^{1}$ ${ }^{1}$ McGill University, Montreal, Quebec; ${ }^{2}$ Tel Aviv Sourasky Medical Center, Tel Aviv, Israel

AIM: Canada and Israel are world leaders in legalizing herbal cannabis for therapeutic reasons. As musculoskeletal complaints are a common reason for persons using medicinal cannabis, rheumatologists' knowledge of cannabinoids was compared in the two countries.
METHODS: All physician members of the Rheumatology Associations of Canada and Israel were surveyed by e-mail for their confidence in knowledge of cannabinoids and their perceived competence to prescribe herbal cannabis.

RESULTS: The response rate for Canada and Israel was 25\% and 20\% respectively. Demographics of the responders from both countries was similar for gender, age, and years in practice, but with more Israeli responders in community/private practice, $\mathrm{P}<0.0001$. Three quarters of responders in both countries were not confident about their knowledge of cannabinoid molecules or ability to write a prescription for herbal cannabis. Differences between country responses for Canada versus Israel were as follows: no role for any cannabinoid treatments, $45 \%$ vs $17 \%, \mathrm{P}=0.019$, never previously recommended trial any cannabinoid, 70 vs $39 \%, \mathrm{P}=0.0075$, some role for pharma or herbal preparations, $30 \%$ versus $48 \%, \mathrm{P}=0.096$, willing to prescribe herbal cannabis if other treatments failed, $28 \%$ vs $83 \% \mathrm{P}<0.0001$. The majority of respondents were not confident to write a prescription for herbal cannabis, $90 \%$ vs $78 \%, \mathrm{P}=0.154$.

CONCLUSIONS: Although rheumatologists in both countries lack confidence in their knowledge of cannabinoids in general, Israeli rheumatologists were more open to cannabinoid treatments with a willingness to prescribe although the majority reported lack of confidence in prescribing the herbal product.

\section{P139}

\section{CHILDKIND: PILOTING AN INTERNATIONAL ORGANIZATIONAL CERTIFICATION PROCESS FOR IMPROVING PAIN IN CHILDREN}

Bonnie Stevens $^{1,2}$, Neil Schechter ${ }^{3,4}$, Paula Forgeron ${ }^{5}$, Gary Walco $^{6,7}$, Allen Finley ${ }^{8,9}$, Jennifer Peleshok ${ }^{1}$, Fiona Campbell ${ }^{1,2}$ ${ }^{1}$ The Hospital for Sick Children; ${ }^{2}$ University of Toronto, Toronto, Ontario; ${ }^{3}$ Boston Children's Hospital; ${ }^{4}$ Harvard University, Boston, Massachusetts, USA; ${ }^{5}$ University of Ottawa, Ottawa, Ontario; ${ }^{6}$ Seattle Children's Hospital; ${ }^{7}$ University of Washington, Seattle, Washington, USA; ${ }^{8}$ IWK Health Centre; ${ }^{9}$ Dalhousie University, Halifax, Nova Scotia

AIM: ChildKind International is a non-governmental organization (incorporated in the US in 2010) aimed at reducing pain and suffering of children globally. The goal is to implement standards of effective treatment of pain in children at the institutional level. Hospitals apply for certification to confirm their practices, policies, and clinical guidelines meet ChildKind criteria and raise the quality of pediatric pain care. This abstract describes the evolution of the process used to evaluate the initial four institutions considered for ChildKind certification and the lessons learned from that process.

METHODS: The pilot certification process was conducted at four pilot sites in North America (Boston Children's Hospital, Connecticut Children's Medical Centre, Seattle Children's Hospital and The Hospital for Sick Children). The process included: a) completing an application form and letter of intent; b) preparing a detailed portfolio; and c) hosting a site visit where four to six members from the ChildKind Executive team reviewed the portfolio, met with executives and leaders of clinical, research and educational pain initiatives and visited selected areas within the institution.

RESULTS: Although all four organizations in the pilot study met the certification standards set out by ChildKind, questions about the process were raised: the essential activities of the site visit, the number of assessors required, the need for mentoring and support, and financial, sustainability and endorsement considerations.

CONCLUSIONS: Piloting an institutional certification process has informed future activities and raised questions for consideration. Ongoing refinement will support the ultimate goal of ChildKind in reducing pain and suffering in children globally. 
P140

VALIDATION OF THE PATIENT-REPORTED HAMILTON INVENTORY FOR COMPLEX REGIONAL PAIN SYNDROME Tara Packham, Joy C MacDermid

School of Rehabilitation Sciences, McMaster University, Hamilton, Ontario

AIM: The Patient-Reported Hamilton Inventory for Complex Regional Pain Syndrome (PR-HI-CRPS) is an outcome measure currently under development. There are currently no widely accepted self-report assessments specific to CRPS.

METHODS: Multi-faceted recruitment (flyers in pain centres, notices in a national CRPS patient support newsletter, and social network postings) was used to recruit 50 persons with CRPS to participate in cognitive debriefing interviews. Readability of the scale and recommendations for additional scale items were judged by the participants as content experts. Interviews were recorded for qualitative analysis of emergent themes about the experience of living with CRPS.

RESULTS: Interm analysis ( $\mathrm{n}=37)$ : Mean age of respondents was 48 years (range 15 to 81 years); M:F 33:4; mean duration of symptoms 69 months (range four to 324); $89 \%$ had been working full-time prior to onset, but only $13.5 \%$ had returned full-time to their job. Items were highly correlated with Cronbach's alpha $=0.93$ for total scale and ranging from 0.72 to 0.87 for the subscales. Participants felt the questions gave a clear overall picture of their daily experience with CRPS but noted two key concepts (depression and temperature/colour changes) were under-represented in the current iteration, and identified problematic wording in several items. Qualitative themes include pain as an invisible disability, the need for better CRPS education for both patients and health professionals, planning skills as essential for coping, and anxiety about the potential impact of their condition on their future health and function.

CONCLUSIONS: Preliminary results generally support the clarity and content of a proposed patient-reported assessment tool for CRPS. Further research is needed to examine the reliability and other forms of validity of the next iteration of the PR-HI-CRPS.

\section{P141 \\ BACK PAIN PREVALENCE AND MANAGEMENT IN ADOLESCENT IDIOPATHIC SCOLIOSIS PATIENTS: A RETROSPECTIVE STUDY}

Lean Théroux ${ }^{1}$, Sylvie Le May ${ }^{1,2}$, Hubert Labelle ${ }^{1,3,4}$

${ }^{1}$ Research Center, Sainte-Justine University Hospital Center;

${ }^{2}$ Faculty of Nursing; ${ }^{3}$ Faculty of Medicine; ${ }^{4}$ Motion Sciences Chair, CHU Ste-Justine and Université de Montréal, Montreal, Quebec

AIM: The aims of this retrospective study were to review randomly selected charts of adolescent idiopathic scoliosis (AIS) patients to describe patients' incidence of back pain (BP) and collect information on its management and treatments.

METHODS: We randomly selected 657 patient files from the "Scoliosys $®$ " database of the CHU Sainte-Justine Hospital between the years 2006 and 2011. Inclusion criteria were: adolescents (10 to 17 years of age) with a diagnosis of IS and documentation of BP. Exclusion criteria were: patients who had a previous scoliosis surgery or any spinal anomalies generating BP. RESULTS: A total of 310 files corresponded to our criteria. Patients' mean $( \pm \mathrm{SD})$ age was $14 \pm 1.78$ years. The most reported single and double curves were respectively thoracolumbar $(31.6 \%)$ and thoracic - thoracolumbar $(21.6 \%)$. BP prevalence was estimated at $47.3 \%$ with the most involved areas being the lumbar (19.7\%) and the thoracic regions $(7.7 \%)$ where the quality of the pain was mentioned in only $21 \%$ of the files. Pain localization could not be ascertained in $64 \%$ of files but narrated terms such as: "Occasional, none - limitative, limitative and generalized pain" were used to describe pain. In approximately $80 \%$ of cases, pain management was not recommended, and follow up on pain treatment could not be determined.

CONCLUSIONS: Back pain prevalence is moderately high among adolescents with spinal deviations. An improved reporting system in regard to $\mathrm{BP}$ evaluation, description, localization, treatment and treatment efficacy would help clinicians better manage this condition in that population.

\section{P142}

BARRIERS TO PAIN AND FEAR MANAGEMENT IN CYSTIC FIBROSIS HEALTH CARE PROVIDERS

(Regular Research Poster)

Student/Trainee Presentation

Rachel M Tomlinson ${ }^{1}$, Amanda Knoll ${ }^{1}$, C Meghan McMurtry ${ }^{1}$, Jean Vinette ${ }^{2}$

${ }^{1}$ University of Guelph, Guelph; ${ }^{2}$ The Hospital for Sick Children, Toronto, Ontario

AIM: This research aimed to understand health care provider perceptions of barriers to effective pain and fear management for patients with cystic fibrosis. Differences in perceptions of barriers between psychosocial and medical health care professionals were also explored.

METHODS: Invitations to participate in an online survey were distributed to health care providers working with adults and children with cystic fibrosis. Health care providers were recruited from cystic fibrosis clinics and listservs across North America.

RESULTS: Participants were 156 health care providers, including nurses (41.7\%), physicians $(16.7 \%)$, dietitians $(14.1 \%)$, child life specialists $(5.1 \%)$ social workers $(5.8 \%)$, and other health professionals $(17.6 \%)$. The top barriers to pain management were: 1) Lack of clinician knowledge about nonpharmacological treatments for pain, 2) Lack of clinician training in pain management, and 3) Belief that some procedure related pain is acceptable without intervention. Analogous results were found for procedural fear.

Psychosocial professionals (eg, social workers) reported stronger concerns regarding certain barriers to pain and fear management than medical health care providers. An example of these includes clinician feelings of vulnerability rising from patient expression of pain $\mathrm{t}(109)=-3.08, \mathrm{P}<0.05$, and fear $\mathrm{t}(110)=-3.15, \mathrm{P}<0.05$.

CONCLUSIONS: Results provide insight into procedures within cystic fibrosis care and how medical and psychosocial providers view barriers to pain and fear management. Results identify direct points of intervention to reduce barriers to pain and fear management in cystic fibrosis.

\section{P143}

PAIN-RELATED BEHAVIORS AMONG CRITICALLY ILL ADULTS WITH AND WITHOUT BRAIN INJURY

\ane Topolovec-Vranic ${ }^{1}$, Celine Gelinas ${ }^{2}$, Jennifer Innis ${ }^{1}$, Yangmei Li ${ }^{1}$, Amanda McFarlan ${ }^{1}$, Sonya Canzian ${ }^{1}$

${ }^{1}$ St Michael's Hospital, Toronto, Ontario; ${ }^{2}$ McGill University, Montreal, Quebec

AIM: In the absence of self-report, observations of pain-related behaviors are recommended as indicators of pain among noncommunicative critically ill patients. The aim of the study was to examine whether critically ill adults with and without brain injuries demonstrate different behaviors during a painful procedure according to the Critical Care Pain Observation Tool (CPOT).

METHODS: Thirty-two patients with brain injuries (Neuro group; $\mathrm{n}=13$ traumatic brain injuries; $\mathrm{n}=19$ aneurysmal subarachnoid hemorrhage) and 15 patients with non-head traumatic injuries (Non-Neuro group) were included in the study. Both communicative $(n=24)$ and noncommunicative patients $(n=23)$ participated. Patients were assessed with the CPOT at rest ( 5 min pre-turning), during turning (a procedure which is known to be painful), and 20 min post-turning. Overall and item scores on the CPOT were compared across the assessments using linear mixed model analyses. RESULTS: Communicative Non-Neuro patients $(n=8)$ self-reported significantly higher pain than the Neuro patients $(n=12)$ across all assessments $(\mathrm{F}=9.6 ; \mathrm{P}=0.003)$. CPOT scores were correspondingly significantly higher for the Non-Neuro patients than the Neuro patients across all assessments (all 47 patients included; $\mathrm{F}=4.3 ; \mathrm{P}=0.041$ ). The differences in the CPOT scores between the two groups were primarily displayed in the muscle tension ( $\mathrm{F}=10.0 ; \mathrm{P}=0.002)$ and vocalization (for communicative patients; $\mathrm{F}=5.1 ; \mathrm{P}=0.029)$ item scores, with Neuro patients demonstrating significantly fewer such behaviors.

CONCLUSIONS: Care must be taken in using observational standardized pain assessment tools with patients with brain injuries. Our findings support previous findings that patients with brain injuries display less muscle tension and vocalization in response to painful stimuli. 
P144

ETHNICITY AND THE PERCEPTION OF PAIN IN OTHERS: A FOCUS ON ABORIGINAL CANADIANS

Kara Turcotte, Susan Holtzman, Ashna Jassi, Alexa Geddes, Courtney Boyer

University of British Columbia-Okanagan, Kelowna, British

Columbia

AIM: To assess the manner in which a label of Aboriginal ethnicity may influence an observers' pain ratings and treatment suggestions.

METHODS: Undergraduate student participants ( $n=355)$ were randomly assigned to read a medical case history vignette of a chronic pain patient who was labeled as either 'Aboriginal Canadian' or 'European Canadian'. The participants then viewed a video of a female adult with chronic pain undergoing a pain induction exercise, and responded to a questionnaire assessing the patient's pain experience such as pain intensity, pain genuineness, and pain-related distress as well as their treatment suggestions. It was hypothesized that the pain experience would be significantly underestimated in patients identified as Aboriginal.

RESULTS: Analyses indicated that the ethnic label did not significantly influence pain intensity estimates $(\mathrm{P}=0.084)$, pain genuineness $(\mathrm{P}=0.198)$ or pain-related distress $(\mathrm{P}=0.484)$. There was also no significant association between ethnicity label and concordance in pain ratings between patients and observers $(\mathrm{P}=0.18)$. Regardless of label, a greater percentage of observers overestimated the patients' pain intensity (53\%) than underestimated pain intensity (10\%). Despite attempts to recruit a diverse sample, only $4 \%$ of the sample identified as Aboriginal (First Nation, Metis, Inuit), so we could not examine the role of ethnicity of the observer.

CONCLUSIONS: Chronic pain and disability is disproportionately prevalent in Aboriginal Canadians as compared to non-Aboriginal Canadians. Emerging evidence indicates that ethnicity may influence the perception of pain in others and contribute to health disparities in minority groups. Future research should investigate factors, such as pain communication, that may contribute to the disparities related to chronic pain in Aboriginal populations with an overall goal of improving cultural competency in pain management.

\section{P145}

\section{THE PERCEPTION OF PAIN IN INDIVIDUALS WITH MAJOR DEPRESSIVE DISORDER: THE MODERATING ROLE OF EMPATHY}

Kara Turcotte, Susan Holtzman, Brittany Boorman, Alexa Rempel University of British Columbia-Okanagan, Kelowna, British Columbia

AIM: To examine the extent to which a label of a comorbid psychiatric condition influences the perception of pain in others as well as the individual differences, specifically trait-level empathy, that may moderate the perception of pain in others.

METHODS: Using a randomized between-subject vignette methodology, the current study examined how a label of comorbid major depression influences the assessment of pain intensity and treatment suggestions in individuals with chronic pain. A sample of undergraduate students $(n=371)$ were randomly assigned to read a vignette of a patient with or without a label of major depressive disorder (MDD) and watched a video of a middle-aged male or female adult with chronic pain undergoing a pain induction exercise. The participants responded to a questionnaire assessing their perception of the patient's pain experience such as pain intensity, pain genuineness, psychological distress, as well as a self-report questionnaire assessing empathy (IRI) and demographic characteristics. It was hypothesized the pain estimates would be lower in individuals attributed with a label of comorbid depression. As well, it was hypothesized that the association between a comorbid depression label and pain estimates would be moderated by trait level empathy, such that individuals with greater empathetic concern would rate patients as being in more pain, compared to those with lower empathetic concern.

RESULTS: Findings indicated that a label of depression in patients with chronic pain influences observers' perception of the patient's psychological distress $\mathrm{t}(369)=-7.01, \mathrm{P}<0.001$, but not pain genuineness or pain intensity (Ps >0.184). No significant moderation effects emerged. Main effects for empathic concern were found where higher levels of empathic concern were associated with higher estimates of patient pain intensity $(\mathrm{B}=-0.16, \mathrm{P}=0.02)$ in the female patient as well as the male patient $(\mathrm{B}=-0.20, \mathrm{P}=<0.00)$.

CONCLUSIONS: Despite research indicating that rates of psychiatric disorders are elevated among chronic pain populations, there is a dearth of research regarding for whom the presence of a comorbid psychiatric condition may influence judgments of pain in others. Given the high rates of comorbid depression in chronic pain patients and the poor quality of life outcomes in this population, future research is needed to examine the manner in which comorbid depression may impact pain perception by formal health care providers.

\section{P146 \\ BIOBEHAVIORAL PAIN RESPONSES IN PRETERM NEONATES UNDER SUCROSE USAGE FOR PAIN RELIEF: EXAMINING SEX DIFFERENCES}

Beatriz Oliveira Valerii ${ }^{1}$, Claudia Maria Gaspardo ${ }^{1}$, Aline Fregni Caetano², Francisco Eulógio Martinez ${ }^{2}$, Maria Beatriz Martins Linhares ${ }^{1}$

${ }^{1}$ Department of Neurosciences and Behavior; ${ }^{2}$ Department of Pediatrics, Ribeirão Preto Medical School, University of São Paulo, Brazil

AIM: To analyze the influence of sex variable on biobehavioral pain reactivity-recovery in preterm infants under usage of sucrose solution for pain relief.

METHODS: Fifty-two neonates born preterm hospitalized in the Neonatal Intensive Care Unit (NICU) were allocated into two groups according to sex variable (Female Group, $F G=27$; Male Group, $M G=25$ ). All infants received the sucrose solution $(25 \%, 0.5 \mathrm{~mL} / \mathrm{kg})$ performed 2 min before acute-painful procedures. Infants' pain biobehavioral reactivity-recovery was assessed during a blood collection procedure divided into the following phases: Baseline (BL), Antisepsis (A), Puncture (P), Recovery-Dressing (D), and Recovery-Resting (R). Behavioral pain reactivity was measured using the Neonatal Facial Coding System (NFCS; pain score range $=0$ to 70); the infants' facial activity was video-recorded. The Sleep-Wake State Scale (SWS; score range $=1$ to 6 ) was assessed at bedside. The heart rate (HR) was continuously recorded, and registered at each minute of the assessment. The comparisons between-groups were performed by MannWhitney test. The level of significance was $5 \%$.

RESULTS: Male and female preterm neonates presented similar biobehavioral pain reactivity-recovery post-puncture pattern in SWS scores and HR responses in all phases of assessment. However, female preterm neonates showed higher NFCS pain scores in the Puncture phase than males (Median NFCS: $\mathrm{FG}=8, \mathrm{MG}=0 ; \mathrm{P}=0.02$ ).

CONCLUSIONS: Preterm neonates under sucrose management for acute-pain relief showed similar pattern of biobehavioral pain reactivityrecovery in SWS and HR parameters, independently of sex. Although female neonates showed more facial activity of pain than male ones in puncture phase, both recovered similarly in subsequent phases of recovery.

\section{P147}

EXPOSURE TO NEONATAL PAIN/STRESS PREDICTS PAIN SENSITIVITY IN SCHOOL-AGE CHILDREN BORN VERY PRETERM

Beatriz Oliveira Valeri ${ }^{1}$, Manon Ranger ${ }^{2,3}$, Cecil MY Chau ${ }^{3}$, Ivan L Cepeda ${ }^{3}$, Anne Synnes ${ }^{2,3,4}$, Maria Beatriz Martins Linhares ${ }^{1}$, Ruth E Grunau ${ }^{2,3,4}$

${ }^{1}$ Department of Neurosciences and Behavior, Ribeirão Preto Medical School, University of São Paulo, Brazil; ${ }^{2}$ Pediatrics, University of British Columbia; ${ }^{3}$ Developmental Neurosciences \& Child Health, Child \& Family Research Institute; ${ }^{4}$ Children's \& Women's Health Centre of British Columbia, Vancouver, British Columbia

AIM: To evaluate whether neonatal pain-related stress is associated with pain ratings to a painful procedure in children born very preterm at school-age. 
METHODS: From a larger longitudinal cohort of infants admitted to a single tertiary NICU, 56 children born very preterm ( $\leq 32$ weeks gestational age $[\mathrm{GA}]$ ) free of major motor, sensory, intellectual impairment or autism, had a blood collection at age 7.7 years (SD 0.4). Children rated their pain to a venipuncture (all received topical anesthetic) using a pain thermometer Color Analog Scale. Mothers completed the Child Behavior Checklist (CBCL) and the State-Trait Anxiety Inventory (STAI). Number of skin-breaking procedures and neonatal measures were obtained from medical/nursing chart review from birth to term-equivalent age. Generalized linear modeling was used to predict pain scores from GA, morphine exposure adjusted for daily weight, infections, number of skinbreaking procedures, CBCL externalizing behavior score, and maternal trait anxiety.

RESULTS: After adjusting for clinical confounders, greater neonatal skinbreaking procedures $(\mathrm{P}=0.02)$ and maternal trait anxiety $(\mathrm{P}=0.003)$ were associated with higher pain intensity ratings during venipuncture. Conversely, higher number of surgeries $(\mathrm{P}=0.006)$ and greater child externalizing behavior $(\mathrm{P}=0.012)$ were associated with lower child pain scores. CONCLUSIONS: In very preterm children without severe sensory, motor or neurodevelopmental impairments, exposure to neonatal pain-related stressful procedures and surgery is associated with altered pain sensitivity at school-age, independent of other neonatal risk factors. Concurrent child externalizing behavior and maternal anxiety level also contribute to children's pain ratings.

\section{P148}

THE EMOTION REGULATORY FUNCTION OF PARENT ATTENTION TO CHILD PAIN AND ASSOCIATED IMPLICATIONS FOR PARENTAL PAIN CONTROL BEHAVIOUR

Tine Vervoort ${ }^{1}$, Line Caes ${ }^{2}$

${ }^{1}$ Department of Experimental-Clinical and Health Psychology, Ghent University, Ghent, Belgium; ${ }^{2}$ IWK Health Centre, Dalhousie University, Halifax, Nova Scotia

AIM: Parents are likely to experience a negative emotional state when faced with their child in pain, often prompting protective behaviour aimed at controlling the child's pain. Ironically, parental pain control behaviors (eg, restricting the child's painful activities) can actually contribute to a child pain and disability. Given the role of parental distress in the occurrence/extent of parental pain control behaviour, it follows that parental ability to regulate pain-related distress may centrally modulate motivational and behavioural outcomes. The present study investigated the function of parental attention in down-regulating distress and pain control behaviour when observing their child engage in an experimental (cold pressor) pain task (CPT), as well as the moderating role of parental anxiety.

METHODS: Participants consisted of a sample of parents and healthy school children ( $\mathrm{n}=62$ parent-child dyads). Parental baseline anxiety was assessed prior to study commencement. Parental attention towards or away from child pain was experimentally manipulated during a viewing task; eye-tracking examined successful compliance with this attention manipulation. Parental ability to regulate distress was assessed using physiological (HR/HRV) and self-report measures. Actual parental pain control behaviour was recorded whilst parents observed their child perform a cold pressor task.

RESULTS: Analyses of eye tracking parameters indicated that the attention manipulation (to attend toward or away from pain) was successful. Further, analysis of (physiological/self-report) emotion regulation indices and pain control behaviour indicated that attentional avoidance versus attention to pain has differential consequences depending upon initial levels of parental anxiety. Specifically, whereas low anxious parents were more distressed and increasingly engaged in pain control behaviour in the attend pain condition, high anxious parents were more distressed and increasingly engaged in pain control behaviour in the avoid pain condition.

CONCLUSIONS: Taken together, the findings of this study indicate that parent attention to child pain is important in understanding emotional and behavioural responses to child's pain. Future research is encouraged to take individual difference variables in account when examining painrelated attention and its consequences.

\section{P149}

EARLY NEONATAL PAIN EXPOSURE AND BRAIN

MICROSTRUCTURE INTERACT TO PREDICT NEURODEVELOPMENTAL OUTCOMES AT 18 MONTHS CORRECTED AGE IN CHILDREN BORN VERY PRETERM

Jillian Vinall ${ }^{1,2}$, Ruth E Grunau ${ }^{1,2,3,4}$, Jill G Zwicker,2,3,5, Vann Chau ${ }^{6,7}$, Kenneth J Poskitt ${ }^{2,3,8}$, Rollin Brant ${ }^{2,9}$, Anne R Synnes ${ }^{2,3,4}$, Steven P Miller ${ }^{3,6,7}$

${ }^{1}$ Neuroscience; ${ }^{2}$ Developmental Neurosciences \& Child Health, Child \& Family Research Institute; ${ }^{3}$ Pediatrics, University of British Columbia; ${ }^{4}$ BC Children's \& Women's Hospitals; ${ }^{5}$ Occupational Science \& Occupational Therapy, University of British Columbia, Vancouver, British Columbia; ${ }^{6}$ Pediatrics, The Hospital for Sick Children; ${ }^{7}$ University of Toronto, Toronto, Ontario; ${ }^{8}$ Radiology; ${ }^{9}$ Statistics, University of British Columbia, Vancouver, British Columbia AIM: Preterm infants are frequently exposed to procedural pain during neonatal care. Greater neonatal pain exposure has been associated with altered brain development. We examined whether the interaction of neonatal pain (number of invasive procedures) and change in brain microstructure over time is associated with neurodevelopmental outcome at 18 months corrected age (CA) in children born very preterm ( $\leq 32$ weeks gestational age [GA]). METHODS: 107 preterm neonates had diffusion tensor imaging at a median 32 weeks (IQR 30.7 to 33.9) and at term-equivalent age (median 40 weeks [IQR 38.7 to 42.6]). Fractional anisotropy (FA), an index of brain maturation, was measured in seven groups of functionally related brain regions. FA change was calculated for each group by subtracting the difference in FA, divided by the number of weeks between scans. Outcome was assessed at 18 months CA using the Bayley-III. Statistical models were adjusted for neonatal and medical confounders.

RESULTS: The interaction between neonatal pain and FA change of the sensorimotor white matter was associated with cognitive, language, and motor outcomes (all $\mathrm{P} \leq 0.02$, adjusted $\mathrm{R}$ squared $\geq 0.17$ ). The interaction between neonatal pain and FA change of the anterior cortical gray matter was associated with motor outcome $(\mathrm{P}=0.05$, adjusted $\mathrm{R}$ squared $=0.30)$. Moreover, the interaction between neonatal pain and FA change of the limbic thalamic gray matter was associated with language outcome $(\mathrm{P}=0.03$, adjusted $\mathrm{R}$ squared $=0.14)$.

CONCLUSIONS: Among preterm infants exposed to greater neonatal pain (adjusted for other risk factors associated with preterm birth), reduced brain maturation during hospitalization was associated with poorer neurodevelopmental outcome at 18 months CA.

Funding: CIHR.

\section{P150}

\section{THE MCGILL CERTIFICATE IN CHRONIC PAIN MANAGEMENT: TWO YEARS EXPERIENCE}

Mark A Ware ${ }^{1}$, Anouk Lamontagne ${ }^{2}$, Celine Gelinas ${ }^{3}$, Kimberley Smalridge ${ }^{2}$, Lesley Singer ${ }^{2}$, Isabelle Gelinas ${ }^{2}$, Maria Drista² ${ }^{2}$, Sara Saunders², Monica Slanik², Annette Majnemer², Robert Dykes ${ }^{2}$

${ }^{1}$ Alan Edwards Pain Management Unit, McGill University Health Centre; ${ }^{2}$ School of Physical and Occupational Therapy; ${ }^{3}$ School of Nursing, McGill University, Montreal, Quebec

AIM: Interdisciplinary postgraduate training in pain management is important for a range of health professionals who encounter chronic pain in their daily practice. In September 2012, McGill University's School of Physical and Occupational Therapy, in collaboration with the Alan Edwards Center for Research on Pain, created an online graduate certificate focused on the most recent evidence-based clinical practices in chronic pain management. We present the first two years experience with our online Graduate Certificate program.

METHODS: A description of the contents of the certificate, the format of teaching and learning styles, the technological requirements, and the demographic and other characteristics of students registering for the 
Certificate over a two year period are described. Descriptions of students experience and feedback with the program were collected.

RESULTS: A total of 37 students have registered to date (Fall 2012: 12; Winter 2013: 2; Fall 2013: 10, Winter 2014: 13). The mean age of students is 34.3 years (range 24 to 52 years), with 27 (73\%) female. The most common discipline for registered students to date is physiotherapy ( $\mathrm{n}=15 \mathrm{stu}-$ dents), occupational therapy ( $\mathrm{n}=11)$, followed by MDs $(\mathrm{n}=5)$, psychologists $(n=2)$ and pharmacists $(n=2)$. Nursing and post-docs made up the remainder $(\mathrm{n}=1$ each). Student testimonials and feedback report high levels of satisfaction with content matter and educational methods.

CONCLUSIONS: Online education is very topical at this time and there is a need to better understand how to work with new information technology to deliver high quality pain education to a range of pain professionals in en effective and interdisciplinary manner. Online programs offer a chance to do this, but there are steep learning curves to setting up and running such courses. We welcome a dialogue between pain educators to determine how to maximize such efforts to improve online inter-professional pain education.

\section{P151 \\ A PAIN EDUCATION INTERPROFESSIONAL WEB-BASED RESOURCE}

Judy Watt-Watson, Michael McGillion, Leila Lax, Judith Hunter, Jon Oskarsson, Cameron MacLennan, Kerry Knickle, Charles Victor University of Toronto, Toronto, Ontario

AIM: To develop and pilot test the effectiveness of an innovative, webbased, pain education interprofessional resource (PEIR) to improve prelicensure health sciences students' knowledge of acute and persistent post-surgical pain assessment and management.

METHODS: Students ( $\mathrm{n}=96)$ were recruited from seven pre-licensure health sciences programs at the University of Toronto: nursing, dentistry, medicine, occupational therapy, pharmacy, physical therapy, social work. Using an iterative design research approach, three interactive PEIR modules were developed, based on an authentic surgical cancer patient case. Case progression spanned pre-operative, post-operative and one-year follow up treatment and pain-related care. Pain mechanisms and key assessment and management issues were incorporated using simulation and cutting-edge virtual, multi-media technology via an accessible software platform. In this interactive format, students worked on appraising evidence and assessment data and engaged with various management options within an interprofessional team. The impact of PEIR on pain and knowledge and beliefs was evaluated pre and post-test using the Pain Beliefs Scale (PBS). Changes in PBS scores were examined using paired $t$ tests. RESULTS: Students $(n=96)$ were recruited from seven pre-licensure health sciences programs at the University of Toronto: nursing, dentistry, medicine, occupational therapy, pharmacy, physical therapy, social work. Using an iterative design research approach, three interactive PEIR modules were developed, based on an authentic surgical cancer patient case. Case progression spanned pre-operative, post-operative and 1-year follow up treatment and pain-related care. Pain mechanisms and key assessment and management issues were incorporated using simulation and cuttingedge virtual, multi-media technology via an accessible software platform. In this interactive format, students worked on appraising evidence and assessment data and engaged with various management options within an interprofessional team. The impact of PEIR on pain and knowledge and beliefs was evaluated pre and post-test using the Pain Beliefs Scale (PBS). Changes in PBS scores were examined using paired t-tests.

CONCLUSIONS: In Canada, only one-third of health science faculties could specify dedicated pain-related content in their curricula. Our pilot results indicate that PEIR is an effective, web-based learning program which improves pain knowledge, significantly. These data support largescale effectiveness testing with a view to implementation, Canada-wide.

\section{P152}

\section{THE ROLE OF PERCEIVED INJUSTICE IN THE PREDICTION OF PAIN AND FUNCTION FOLLOWING TOTAL KNEE ARTHROPLASTY}

Esther Yakobov ${ }^{1}$, Whitney Scott ${ }^{1}$, William Stanish ${ }^{2}$, Michael Dunbar ${ }^{2}$, Heather Adams ${ }^{3}$, Michael Sullivan ${ }^{1}$

${ }^{1}$ McGill University, Montreal, Quebec; ${ }^{2}$ Dalhousie University, Halifax, Nova Scotia; ${ }^{3}$ McGill University Health Centre, Montreal, Quebec

AIM: The primary aim of this study was to investigate the role of perceived injustice in prediction of pain severity and disability following Total Knee Arthroplasty (TKA).

METHODS: The study sample consisted of 116 ( 71 women, 45 men) individuals with osteoarthritis of the knee scheduled for TKA at one of three hospitals in eastern Canada. Participants completed measures of pain severity, physical disability, perceptions of injustice, pain catastrophizing, and fear of movement immediately before surgery and one year later.

RESULTS: Consistent with previous research, cross-sectional analyses revealed significant bivariate correlations between psychological variables, and measures of pain severity and disability. Prospective multivariate analyses revealed that perceived injustice contributed significant unique variance to the prediction of post-surgical pain severity, and pain catastrophizing contributed significant unique variance to the prediction of postsurgical disability.

CONCLUSIONS: The current findings add to the growing research indicating the prognostic value of perceived injustice in the prediction of painrelated outcomes. The present results suggest that assessment of perceived injustice in the pre-surgical screening of individuals scheduled for TKA might permit identification of individuals at-risk for problematic surgical outcomes. Psychosocial interventions designed to target perceptions of injustice and pain catastrophizing before surgery may be useful in the prevention of chronic pain and disability following TKA.

\section{P153}

CANCER-RELATED NEUROPATHIC PAIN: AN INVESTIGATION OF PAIN QUALITIES AND IMPACT Alycia Young ${ }^{1}$, Lynn R Gauthier ${ }^{1,2}$, Robert H Dworkin ${ }^{3}$, Ronald Melzack ${ }^{4}$, Gary Rodin, ${ }^{2,5}$, Camilla Zimmermannn, David Warr $^{2,5}$, S Lawrence Librach ${ }^{5,6}$, Malcolm Moore ${ }^{2,5}$, Frances A Shepherd ${ }^{2,5}$, Lucia Gagliese ${ }^{1,2,5}$

${ }^{1}$ York University; ${ }^{2}$ University Health Network, Toronto, Ontario; ${ }^{3}$ University of Rochester, Rochester, New York, USA; ${ }^{4}$ McGill University, Montreal, Quebec; ${ }^{5}$ University of Toronto; ${ }^{6}$ Mount Sinai Hospital, Toronto, Ontario

AIM: Although up to $40 \%$ of people with cancer may develop neuropathic pain $(\mathrm{NeP})$ as part of the disease itself or as a consequence of treatment, little is known about its characteristics or impact. The purpose of this study was to compare individuals with cancer-related $\mathrm{NeP}$ to individuwals with non-neuropathic cancer pain.

METHODS: 267 patients ( $58 \%$ female; average age: $58 \pm 11.7$ years of age) with advanced cancer attending outpatient clinics or receiving home palliative care completed questionnaires pertaining to disease, treatment, pain, mood and demographics. Two groups were formed based on responses to the NeP Subscale of the Short-Form McGill Pain Questionnaire-2: NeP $(\geq 1 \mathrm{NeP}$ descriptor selected; $\mathrm{n}=226$ [85\%]) and non-NEP (0 NeP descriptors selected; $n=41[15 \%])$.

RESULTS: The NeP group had significantly worse physical functioning (26.8 \pm 13.8 versus $34.2 \pm 19.6 ; \mathrm{P}=0.004)$ and symptom distress $(37.3 \pm 17$ versus $31.0 \pm 16.9 ; \mathrm{P}=0.048)$ than the non-NeP group. Pain location also differed: $22 \%$ of $\mathrm{NeP}$ versus $0 \%$ of non-NeP reported peripheral pain. Patients with peripheral NeP including "hot-burning" sensations $(n=29)$ had greater symptom distress $(45.53 \pm 20.9$ versus $28.05 \pm 12.7 ; \mathrm{P}=0.002)$, pain intensity $(4.77 \pm 2.1$ versus $3.19 \pm 1.4 ; \mathrm{P}=0.005)$ and pain interference $(5.58 \pm 2.4$ versus $4.29 \pm 1.7 ; \mathrm{P}=0.043)$ than patients with peripheral $\mathrm{NeP}$ who did not select "hot-burning".

CONCLUSIONS: Neuropathic cancer pain is associated with worse quality of life (QOL) and greater symptom distress than non-neuropathic 
cancer pain. Hot-burning peripheral $\mathrm{NeP}$ may be especially impactful. Further research is needed on individuals with $\mathrm{NeP}$ in order to improve pain management and QOL.

\section{P154}

PAIN CHARACTERISTICS AND OPIOID CONSUMPTION IN SICKLE CELL DISEASE PATIENTS REFERRED TO A TERTIARY PAIN CLINIC

Nivan Zoheiry, Maha Alkokani, Angela Mailis-Gagnon

Comprehensive Pain Program, Toronto Western Hospital/University Health Network, Toronto, Ontario

AIM: To describe the characteristics of patients with Sickle Cell Disease (SCD) and chronic pain referred to a tertiary care pain clinic.

METHODS: This is a cross-sectional descriptive study. Demographics, pain ratings and opioid use data were collected via retrospective chart review Data from 16 consecutive SCD patients referred from the hospital's Haematology service over a four year period.

RESULTS: All patients reported acute painful crises of variable intensity at early age. By the time of referral to us, all were in constant pain (mean 5.28/10 during interview; range $1 / 10$ to $9 / 10$ ). Female/male ratio was $2.2: 1$, mean age 29.5 years; mean chronic pain duration 5.25 years; $62.5 \%$ were Canadian born primarily of African or Caribbean decent; $68.8 \%$ were unemployed, and $81.3 \%$ were receiving disability payments. All but two patients (14 of 16 [87.5\%]) were on different opioids, with mean Morphine Equivalent Daily (MED) $216.6 \mathrm{mg}$ (range $60 \mathrm{mg}$ to $560 \mathrm{mg}$ ); $35.7 \%$ of the opioid users were receiving $>200 \mathrm{mg}$, the suggested 'watchful dose' of the 2010 Canadian Guideline for safe and effective use of opioids in Chronic Non Cancer Pain (range $202 \mathrm{mg}$ to $560 \mathrm{mg}$ ).

CONCLUSIONS: The pain team found that most SCD patients on opioids could be managed with non opioid treatments (except at the time of crisis) and suggested tapering and/or discontinuation of opioids in eight opioid users due to absence of physical findings; modification/ upwards titration of the opioids in three patients with serious pathology; and maintenance of three patients in low dose prn opioids, while added antidepressants in half of the opioid users. 


\section{A}

Abdelaziz DM. .................. P2

Abdolmohammadi S .............. P3

Abdulla K ....................... P83

Abdullah S ................... P2

Ablin J ..................... P138

Adams B . . . . . ............. P21

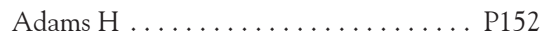

Agarwal A .................... P90

Agarwal G . . . . . . . . . . . . . . . 16B

Ahola Kohut S. . . . . . . . . . . 9D,P121

Alkokani M .................P4,P154

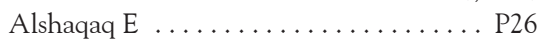

Amariglio N ................. P5

Ambuel B .................... P80

Anastas T ................... P65

Andersen RD .................. P80

Andreae M .................. 23B

Arbour C . . . ....... P7,P8,P20,P9,P82

Archibald K ................. P91

Arsenault P . . ................... P11

Awad IT ..................... P34

Awad I . . . . . . . . . . . . . . . . . . P35

Ayling OGS.................. P113

\section{B}

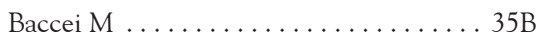

Baerg K ................. P13

Bagby $\mathrm{M} \ldots \ldots \ldots \ldots \ldots \ldots \ldots$. . . . . . . .

Bailey B . . . . . . . . . . . . . P103

Bailey K . . . . . . . . . . . . . . . . P12

Balaton B . . . . . . . . . . . . . . P13

Balon J . . . . . . . .............. P11

Bance $S \ldots \ldots \ldots \ldots \ldots \ldots \ldots$. . . . . . . . 27

Baril AA ................... P9

Barrowman N ................ P83

Barton P .................... 38C

Batra YK ................... P14

Bearden DJ.................. P132

Beaulieu LD ................. 16C

Beaulieu-Boire L . . . . . . . . . . . P100

Beggs RT .................. P15

Beggs $S \ldots \ldots \ldots \ldots \ldots . \ldots 31 \mathrm{C}, 35,35 \mathrm{~A}$

Beier F . . . . . . . . . . . . . . . . P69

Belfer I . . . . . . . . . . . . . . 25B

Bennett SM .................. P16

Benoit B . . . . . . . . . . . P17,P45

Bergman J . . . . . . . . . . . . . 33A

Bernier E . . . . . . . . . . . . . . P135

Berthiaume $Y \ldots \ldots \ldots \ldots \ldots \ldots$. . . . . . . . 89

Bird S ................... P12

Birnie KA. ............. 16A,P18

Blaise $G \ldots \ldots \ldots \ldots \ldots \ldots \ldots$. . . . . . . . .

Bletcher P .................. P11

Boerner KE. . . . . . . . . . . 6 6,16A

Boisvert M . . . . . . . . . . . . . . . . P19

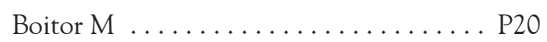

Boivin M .................. 10A

Boorman B ................. P145

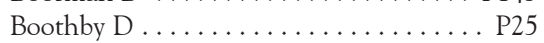

Bouchard M ................. P21

Boulander A .................. P114

Bourgault P . . . P P19,P22,P64,P99,P100,P125

Boyer C . . . . . . . . . . . . . . . . . . P144

Boyle J .................. 18A,26C

Boyle MH ............... 16I,P92

Brant R . . . . . . . . . . . . . . . . . P149
Breau L .................... P59

Bremner T ................. 7B

Bromberg $\mathrm{MH} \ldots \ldots \ldots \ldots \ldots \ldots . . \ldots 23$

Browne E ................... P24

Brunati R ................. P112

Buckley N . . . . . . . . . . . . . P26,P56

Bueno M .................... P84

Burcher A ................. P42

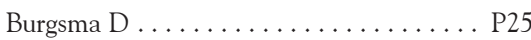

Busse JW ............ P26,P27,P28

Buzon-Tan A . . .............9B,P10

\section{C}

Cabana F .................. P87

Caddell K .................. P17

Caes L . . . . . . . . . . 2,2A,16A,P29,P148

Caetano AF . . . . . . . . . . . . . . . P146

Cairns B ................. 19,19C

Campbell F . . . . . . . . P30,P126,P139

Campbell L . . . . . . . . . . . . . . . P105,P106

Campbell-Yeo M . .9,24B,31,31B,P17,P45,P122

Canzian S .................. P143

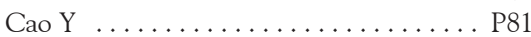

Carlson T ................. P55

Carnide $\mathrm{N} \ldots \ldots \ldots \ldots \ldots \ldots$. P56

Carol A ..................... P26

Carr E ....................... 31

Carr LK ................... P94,P115

Carrière JS .................. P32

Cepeda IL. . . . . . . . . . . . . . . P147

Cervero F ................3,3A

Chambers CT. . . . . . 3B,15,16A,20C,30,P18,

..................... P29,P134

Charest MC $\ldots \ldots \ldots \ldots \ldots \ldots \ldots . . . . . .2103$

Charette S .................. P103

Chau CMY...................P33,P147

Chau V ..................... P149

Chedore B ................... 11C

Chesworth B . . . . . . . . . . . . . . . . P69

Chin K ................... P133

Choinière M ........27B,P7,P8,P89,P99

Chorney J . . . . . . . . . . . . . P12,P55,P63,P91

Chouinard G . ................ P11

Clark AJ..................P41,P114

Clarke H . . . . . 23C,P34,P35,P95,P113,P114

....................P123,P124

Clarke $S \ldots \ldots \ldots \ldots \ldots \ldots \ldots . . \ldots 116$

Clauw DJ ...................... P54

Cloutier C . . . . . .................... P11,P22

Cohen LL. . . . . . . . . . . . . . . . . . . P132

Cohen L . . . . . . . . . . . . . . . . . . . . P36,P108

Cohen N ...................P123,P124

Coleman RM ................. P64

Cooper L . . . . . . . . . . . . . 11A,P26

Costigan M .................. 35C

Courtemanche-Harel R ..........P99,P100

Cousins $\mathrm{L} \ldots \ldots \ldots \ldots \ldots \ldots \ldots$. P36

Creary.................... P,P39

Crump L .................. P37

Cruz MDD ................. P38

Cuello AC . . . . . . . . . . . . . 34A,P107

Cumber J . . . . . . . . . . . . . . . . . P83

Cunningham N . . . . . . . . . . . P23

Curtis K ................ P123,P124

\section{$\mathrm{D}$}

Dalley J P39

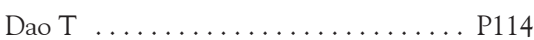

Dart R .................. 22B

Davies WH. ................. P81

De Koninck Y . . . . . . . . . . . . . . . 34C

de Levie A ................... P16

de Oliveira CR. . . . . . . . . . . . . . . P38

de Rivero Gutierrez MG . . . . . . . . . . . P62

Debigaré $\mathrm{R} \ldots \ldots \ldots \ldots \ldots \ldots$ 9,P73

Deits C .................... P65

DeLongis A .................. P15

DEntrement MA ............... P41

Desjardine K ................ P42

Devlin A .................. P33

Dhaliwal M .................. P30

Dhutt SS ................... P14

Dias CG .................... P62

Diatchenko L $\ldots \ldots \ldots \ldots .25,25 \mathrm{~A}, 32 \mathrm{C}$

Dick BD . . . . . . . . . . . . . . . . . 16, P16

Dick B ............ 8C,P55,P118

Dikareva $S \ldots . \ldots \ldots \ldots \ldots \ldots \ldots$. . . . . . . 31

Dionne CE.................. P74

Ditto B ................6.

Doca FNP. ................ P43,P44

Doggweiler R ................. P115

Dol J ...................... P45

Dolovich L . . . . . . . . . . . . . 16B

Donworth M ................. P11

Drista $M$.................... P150

Dubin RE .................. 18,18C

Dubin R.................. $7 \mathrm{C}$

Duclos C ..................... P9

Duhigg D .................. 18B

Dumas M .................. P102

Dunbar M ................... P152

Durrence HH. . . . . . . . . . . . . 9C,P85

Dworkin RH ............... P58,P153

Dykes R . . . . . . . . . . . . . . P150

\section{$\mathrm{E}$}

Ebrahim S ..............P27,P28

Eccleston C . . . . . . . . . . . . . P72

Egan J ................ 9A,P6

Eisenberg $\mathrm{N} \ldots \ldots \ldots \ldots \ldots \ldots \ldots . . \ldots 113$

Eisenhoffer J ................ P11

Essner B . . . . . . . . . . . . . . . . . . P66

Etheridge $\mathrm{P} \ldots \ldots \ldots \ldots \ldots \ldots \ldots \ldots$

Evans JR................... P81

\section{$\mathrm{F}$}

Fashler SR .................. P47

Ferland CE $\ldots \ldots \ldots \ldots \ldots \ldots \ldots .21,21 \mathrm{~A}$

Fernandes AM . . . . . . . . . . . . P38

Fernandes A .................. P50

Ferrante S ................. P112

Ferreira de Campos DC . . . . . . . . . . P50

Fesinmeyer M ................ P66

Finley A ............ P43,P44,P139

Finley GA $\ldots \ldots \ldots \ldots \ldots \ldots \ldots$ P114

Fiola JL..................... P103

Fitzcharles MA ... P53,P54,P110,P129,P138

Fleischman $\mathrm{K} \ldots \ldots \ldots \ldots \ldots . . . . . .2110$

Forgeron PA................. P55

Forgeron P . . . . . . . . . . P P91,P139

Fortune $\mathrm{M} \ldots \ldots \ldots \ldots \ldots \ldots \ldots$ P56

Foty R ................. P133

Fowler MJ.................. P26

Fox E ................... P72 
Frizzell B .................16F

Fuller A . . . . . . . . . . . . . . P83

Furlan A ................. P56,P114

Fuss $\mathrm{S} \ldots \ldots \ldots \ldots \ldots \ldots \ldots$. P124

\section{G}

Gagliese $L \ldots \ldots \ldots \ldots \ldots$ 2,2C,P58,P153

Gagnon AM. .................. P4

Gagnon MM ........... P46,P57,P70

Gallagher F . . . . . . . . . . . . . . P19

Gandhi W ................... P29

Garfield H . . . . . . . . . . . . . . P105,P106

Garland R ................ P135

Garofalo L . . . . . . . . . . . . . . . 22C

Gaspardo CM. . . . . . . . . . . . . . . . P146

Gaudreault PO ................ P9

Gauthier LR. . . . . . . . . . . . . . P58,P153

Geddes A ................... P144

Gelinas C ................ P143,P150

Gelinas I . . . . . . . . . . . . . . P150

Gélinas C ......... 15,P7,P8,P20,P82

Genik L . . . . . . . . . . . . . . P P59

Gennis HG ................. P134

Gereau IV RW . . . . . . . . . . . . . . . . . 14

Germain K ................. P25

Giladi $H \ldots \ldots \ldots \ldots \ldots \ldots$ P60,P61

Gillis J . . . . ................ P11

Gilron A ................... P114

Gilron I ................... 4B

Ginting J . . . . . . . . . . . P115

Giuliani F .................. P118

Glowacka M ................2, B

Gold JI . . . . . . . . . . . . P5,P110,P111

Gold J .................... P109

Gollish J ................. P34,P35

Gonzalez IB . . . . . . . . . . . . . . . P109

Gordon AS . . . . . . . . . . . . . 37B

Gordon A .................. P114

Gorodzinsky AY ................. P63

Gosselin É . . . . . . . . . . . . . . . . . . . P64

Gosselin N ................. P9

Grant D . . . . . . . . . . . . . . . . . . P95

Greenberg S . . . . . . . . . . . P105,P106

Grewal S ................... P65

Groenewald CB . . . . . . . . . . . . . P P66

Grunau RE . . . . . . . . . 21,21B,P33,P149

Grunau R ................... 31A

Grunau RE ................. P147

Gullickson KM ................ P67

Guyatt GH.............. P27,P28

\section{$\mathrm{H}$}

Hadjistavropoulos T . . . 3C,17,17B,P37,P46,

$\ldots \ldots \ldots \ldots \ldots \ldots \ldots \ldots \ldots \ldots \ldots \ldots \ldots$

Hagel J . . . . . . . . . . . . . . . . P68

Hainsworth KR ................. P81

Hamilton CB . . . . . . . . . . . . . . . . P69

Hampton ADJ . . . . . . . . . . . . . . P70

Hampton A . . . . . . . . . . . . . . . P46, P57

Hanley J . . . ................. P126

Harman K . . . . . . . . . . . . . . . 5B,P37

Harrison D ................ P83,P84

Harrison J . .................. P71

Harsanyi Z . . . . . . . . . . . . . . P11

Haslam L ................ P133

Heathcote $\mathrm{LC} \ldots \ldots \ldots \ldots \ldots \ldots \ldots$ P72

Heels-Ansdell D ............... P28

Hegarty D .................. P68

Henderson EM................. 36A
Henderson PR ................. P96

Henein $S \ldots \ldots \ldots \ldots \ldots \ldots \ldots$ P11

Henry JL. . . . . . . . . . . . . . . . . . P101

Hewitt B .................. P122

Higgins KS . . . . . . . . . . . . . P134

Higgins $\mathrm{NC} \ldots \ldots \ldots \ldots \ldots \ldots \ldots . . \ldots \ldots$

Hildebrand ME ............... 20B

Hillard CJ......................28

Hohmann AG . . . . . . . . . . . . . . . . . 28

Holmbeck G . . . . . . . . . . . . . . 16D

Holtzman S . . . . . . . . P15,P144,P145

Hooper RA. . . . . . . . . . . . . . . 16F

Houde F . . . . . . . . . . . . . . P87

Hunter J ................... P151

\section{I}

Iliza AC . . . ................... P89

Innis $\mathrm{J} \ldots \ldots \ldots \ldots \ldots \ldots \ldots \ldots$. . . . . . . . . . . .

Irvin $\mathrm{E} \ldots \ldots \ldots \ldots \ldots \ldots \ldots \ldots \ldots \ldots \ldots \ldots$

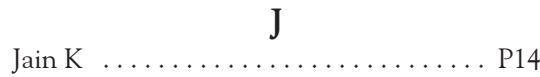

Jamal S ................... P54

Jassi A ................... P144

Jastrowski Mano KE. .............. P81

Jeanneret A . . . . . . . . . . . . . . . P89

Jiang J .................. P113

Jibb L .................... P90

Joffe NE .................... P132

Johnston C ...............P17,P45

Jones $\mathrm{K} \ldots \ldots \ldots \ldots \ldots \ldots \ldots$. . . . . . . . 91

Jordan AL ................. P23

Jovey RD . . . . . . . . . . 10,10B,13,37,37A

Julien N . . . . . . . . . . . . . . . . . . 16G,P88,P93

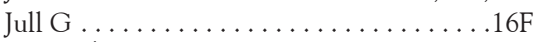

Junior ÁLC . . . . . . . . . . . . . P P 3,P44

Jylli L ....................... P80

\section{K}

Kaasalainen S .................. 16B

Kais $\mathrm{L} \ldots \ldots \ldots \ldots \ldots \ldots \ldots \ldots \ldots$ 16

Karsh J .................... P54

Kaseweter K ................. P24

Kashikar-Zuck S ............. P23

Katz J . . 11,23,23A,27,P34,P35,P47,P95,P113

$\ldots \ldots \ldots \ldots \ldots \ldots . . \ldots$ P123,P124,P133

Katz L . . . . . . . . . . . . . P94,P97,P115

Kay J .................... P34,P35

Kelly A ................... P11

Kennedy D ................ P34,P35

Khadr C .................... P103

Khan J ..................... P95

Khan KA .................. P81

Khouri L ................... P82

Kim J .................. P56

Kirkham K .................9B,P10

Kisely SR . . . . . . . . . . . . . . . P18

Klinger L . . . . . . . . . . . . . . . P25

Knickle K ................. P151

Knoll A ..................... P142

Ko R ................... P95

Kobylecka M ................ P111

Komarova SV ................. P2

Kowal J ................... P96

Krancevic AM .............P30,P126

Kreder H ................. P133

Krsmanovic A . . . . . . . . . P94,P97,P115

Kumar B ................ P14
Kumbhare D ................ P101

Kunz R.................... P27

\section{$\mathrm{L}$}

Labelle H................... P141

Laberge D . . . . . . . . . . . . . . P98

Lacasse A ............ 16G,P88,P93,P99

LaChapelle DL................. P37,P67

Ladak S . . . . . . . . . . . . . . . . . P95,P113

Ladwig R . . . . . . . . . . . . . . P81

Lafrenaye S .......... P22,P102,P125

Lagueux E ................... P100

Laird KT. .................. P136

Lalloo $\mathrm{C} \ldots \ldots \ldots \ldots \ldots \ldots$ P90,P101

Lamb L . . . . . . . . . . . . . . . . . P60

Lamontagne A ............... P150

Lapré J ..................... P22

Larochette AC ............... P16

Latimer $\mathrm{M} \ldots \ldots \ldots \ldots \ldots \ldots \ldots$ P17

Lau JYF ...................... P72

Lavigne GJ . . . . . . . . . . . . . 32B,P9

Lavoie $S \ldots \ldots \ldots \ldots \ldots \ldots \ldots$. P64

Lavoie-Pilote AM ............... P89

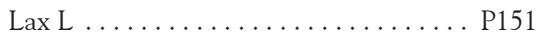

Le TTH ..................... P102

LeBlanc A ................... P126

LeClercq $S \ldots \ldots \ldots \ldots \ldots \ldots \ldots$ P54

LeFort $S \ldots \ldots \ldots \ldots \ldots \ldots \ldots \ldots$. P104

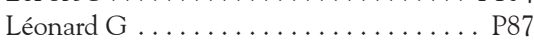

Lesage MA $\ldots \ldots \ldots \ldots \ldots \ldots \ldots$. 222

Levy D ..................... 19B

Li Y ..................... P143

Librach SL ................ P58,P153

Lidster N . . . . . . . . . . . . . . P26

Linhares MBM. . . . . . . . P43,P4,P119,P120,

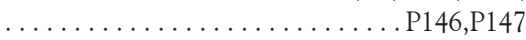

Lisi D ................... P105,P106

Litwin MS ................. P97

Loiselle CG . ............... P7,P8

Love $\mathrm{L} \ldots \ldots \ldots \ldots \ldots \ldots \ldots \ldots$. P113

Lynch ME. .................. 27,P41

Lynch M ..................17A

\section{$\mathbf{M}$}

MacDermid JC . . . . . . . . . 16E,16H,P140

MacDougall P. ................. P11

MacKay J ................... P12

MacLennan C . ............... P151

Macpherson A ............... P58

Maddelena V ................... P104

Magnussen C ................... P107

Mahmood H ................. P26

Mailis-Gagnon A .............. P154

Majnemer A ................... P150

Makkar JK .................... P14

Maqbool A .................. P26

Maqbool B ................... P26

Martens Van Hilst Y . . . . . . . . . . . 33B

Martin S ................. P108

Martinez FE ................. P146

Martorella G .................. P20

Massé-Alarie H .................. 16C

Mathieu N . . . . . . . . . . . . . . . . . 9,P73

May SL.................. P103,P141

Mayer R ..................P94,P115

Mayer $S \ldots \ldots \ldots \ldots \ldots \ldots \ldots . . . \ldots 22$

McCartney CJL ................. P133

McCartney C . . . . . . . . . . P34,P35

McCluskey S ................. P95 
McDougall JJ ................... P54

McEvoy A ................... P135

McFarlan A .................... P143

McFetridge-Durdle J . . . . . . . . . . . P116

McGillion M . .........12,12B,P116,P151

McGinnis E . . . . . . . . . . . P26

McGrath PJ . . . . . . . . . . . . . . P18,P132

McGrath P . . . . . . . . . 36,P12,P122

McGuire B ............9A,36B,P6

McMurtry CM . . . . . . . . . . . P39,P59,P142

McNair C .................. P126

McNaughton-Collins MF . . . . . . . . . P97

McWilliams LA ................. P96

Melzack R ...............P58,P153

Mercier C . .................. P98

Meyer RML ............ P5,P110,P111

Meyer R . . . . . . . . . . . . . . . . . P109

Michalko K .................. P11

Michaud Cé .................. P20

Milioto M .................. P135

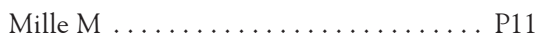

Miller SP ...................... P149

Miró J ..................... P137

Mithal P .................. P106

Moffet H . . . . . . . . . . . . . 9,P73

Mogil JS ................1,14,25C,29

Mohammed A . ............... P26

Moldwin R .................. P115

Montbriand J . . . . . . . . . . . . . P113

Montgomery L .....................33

Monticone M ............... P112

Moon EC . . . . . . . . . . . . . . . . P16

Moore M ................P58,P153

Morin D . . . . . . . . . . . . . . P74

Morley-Forster PK . . . . . . . . . . . . P114

Moro J ..................... 16H

Moseley GL . . . . . . . . . . . . . . . . . . 1

Moulin DE ................. P114

Muere A .............. P94,P97,P115

Munhall C................. P56

Murphy M .................. P104

Murray C . ................... 16D

\section{$\mathrm{N}$}

Nanji M ................... P126

Nascimento F . . . . . . . . . . . . . P107

Nelson S ................... P116

Nicholls S ..................... P83

Nickel CJ . . . . . . . . . . . . . . . . . . P94

Nickel JC . . . . . . . . . . . . . . . P97,P115

Noel M ................. P18

\section{$\mathrm{O}$}

O'Keefe-McCarthy S . . . . . . . P116,P117

O'Mahony W ................ P11

O'Neill M ..................9D,P121

Oaklander AL ...................... . 29

Oberlander TF................ P33

Olechowski C . . ............. P118

Oliveira NCAC. . . . . . . . . . . . P119,P120

Orr T .............. P17,P45,P122

Oster H . . . . . . . . . . 9D,P121

\section{P}

Packham T ................. 16E,P140

Page $G \ldots \ldots \ldots \ldots \ldots \ldots$ 27A,P35

Pagé MG ............. P34,P123,P124

Pais MJC ............... P50
Palermo T . . . . . . . . . . . . . . 16D,P66

Parker JA $\ldots \ldots \ldots \ldots \ldots \ldots$ P18,P29,P134

Paul-Savoie E ................. P125

Pearson N ..................... 5C

Peleshok J . ........... P30,P126,P139

Pepper J ................... P30

Perreault K .............9,P73,P74,P98

Pest M . ................... P69

Peugh J .................. P23

Pillai Riddell R. . . 9D,23,23A,P58,P105,P106

..................... P121,P128

Piraino $\mathrm{P} \ldots \ldots \ldots \ldots \ldots \ldots \ldots \ldots \ldots \ldots$

Pitelka V . . . . . . . . . . . . . . . . P69

Plante E . . . . . . . . . . . . . . . . P55

Poitras $S \ldots \ldots \ldots \ldots \ldots \ldots . . \ldots$ P74

Pontari M .................. P97

PORSCHE Study Group . . . . . . . . . . P63

Poskitt KJ . . . . . . . . . . . . . . . . . P149

Potvin $S \ldots \ldots \ldots \ldots \ldots \ldots \ldots$ P125

Pound $\mathrm{C} \ldots \ldots \ldots \ldots \ldots \ldots$. . . 83

Preuss R .................... 16C

Prkachin $\mathrm{K} \ldots \ldots \ldots \ldots \ldots \ldots \ldots$ P24

Puntillo KA ................... P8

\section{$\mathbf{R}$}

Racine N . ................. P128

Rampakakis E .............. P53,P129

Ranger M ............... P33,P147

Rashiq S ................. 8,27B

Ratneswaran A . . . . . . . . . . . . . P69

Rauch F ................... P2

Ready L . . . . . . . . . . . . . . . . . P117

Rempel A ................. P145

Reszel J . . . . . . . . . . . . . . . . . . P83,P84

Rheault P ................ P11

Ribeiro-da-Silva A . . . . . . 34,34B,P107

Ritcey D .................. P29

Riva JJ ................... P27

Robinson $\mathrm{L} \ldots \ldots \ldots \ldots \ldots \ldots \ldots$. P21

Rocca B .................. P112

Roche-Nagle G ............... P113

Rod K ..................9E,P131

Rodin $G \ldots \ldots \ldots \ldots \ldots \ldots$ P58,P153

Rodrigues $\mathrm{N} \ldots \ldots \ldots \ldots \ldots \ldots$. P132

Romero Escobar EM . . . . . . . . . P123,P124

Rosenbaum D ................ P26

Rosenbloom BN................ P133

Ross E .................... P47

Ross M .............. P7,P8,P82

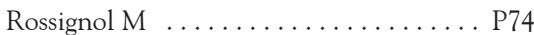

Roux JF .................9,P73

Roy $\mathrm{F} \ldots \ldots \ldots \ldots \ldots \ldots \ldots \ldots \ldots \ldots \ldots$

\section{$\mathrm{S}$}

Sadri P . ................... P11

Saigal S .................. 16I,P92

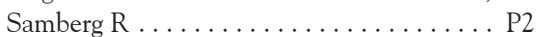

Sampalis JS ............... P53,P129

Sampson M .................. P83

Saunders S . . . . . . . . . . . . . P150

Savard I . . . . . . . . . . . . . . . 9,P73

Schacter $G \ldots \ldots \ldots \ldots \ldots \ldots \ldots$. P11

Schechter N ................. P139

Schinkel MG . . . . . . . . . . . . . . . 16A,P134

Schmidt LA . . . . . . . . . . . . . . . . . . 16I,P92

Schneider C ................. 16C

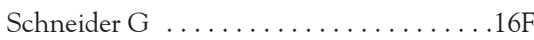

Schweinhardt P . . . . . . ......... P29

Scott W . . . . . . . 20,38B,P61,P135,P152
Senior A $\ldots \ldots \ldots \ldots \ldots \ldots \ldots \ldots$. 25

Sessle B .................... 19A

Sessle BJ .............. 32,32A,P114

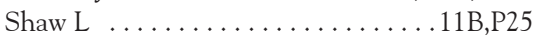

Shepherd FA . . . . . . . . . . . P P58,P153

Sherman AL ................ P136

Shir Y ..... P53,P54,P61,P60,P129,P138

Shirkey KC ............... P136

Shojania K .................. P54

Shoskes D .................. P97

Shram MJ................22, 22A

Shripts D ................... P82

Sil S ..................... P23

Silton R . . . . . . . . . . . . 16D

Simmonds M .................... 8A

Singer $\mathrm{L} \ldots \ldots \ldots \ldots \ldots \ldots \ldots$. . . . . . . . . . . . . . . . . .

Slanik M ................... P150

Smalridge K ................. P150

Smith A $\ldots \ldots \ldots \ldots \ldots \ldots$ 16F,38,38A

Smith CA ................. P136

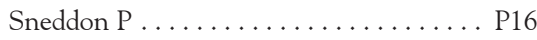

Solé E .................... P137

Squire P . . . . . . . . . . . . . . . 13, P114

Srinivas C . . . . . . . . . . . . . P95

St-Amant H ............16G,P88,P93

Stanish W .................... P152

Steenstra I . . . . . . . . . . . . . . P28

Ste-Marie P . . . . . . . . . . . . . . . . P138

Ste-Marie PA . . . . . . . . . . . P54,P53,P129

Sterling M . . . . . . . . . . . . . . . 16F

Stevens B .........30,P30,P126,P139

Stinson J . . 17C,P30,P46,P57,P90,P114,P122

Stone $\mathrm{LS} \ldots \ldots \ldots \ldots \ldots \ldots . \ldots \ldots, 4 \mathrm{~A}, \mathrm{P} 2$

Stratford P . . . . . . . . . . . . . P34,P35

Strotman D . . . . ............. P23

Sullivan MJL . . . . . ..............P32,P61,P135

Sullivan M ...............P71,P152

Sumpton J . ................. P26

Symons F . . . . . . . . . . . . . . 21C

Synnes A . . . . . . . . . . . . . . . . . . P147

Synnes AR.................... P149

\section{$\mathrm{T}$}

Taddio A . . . . . . .6,6A,12A,24C,P128

Taenzer P . . . . . . . . . . . . . . . . . P114

$\operatorname{Tan} \mathrm{X} \ldots \ldots \ldots \ldots \ldots \ldots \ldots \ldots . . \ldots 60$

Théroux J ................. P141

Thiffault R ................... P22

Thompson EN . . . . . . . . . . . . . . . 26,26A

Ting TV .................. P23

Tomlinson RM................ P142

Topolovec-Vranic J . ........P7,P8,P82,P143

Toth C . . . . . . . . . . . . . . . . . . P114

Tousignant-Laflamme Y .......... P100

Traynor A ................ 9A,P6

Tripp DA ............. P94,P97,P115

Trost Z . . . . . . . . . . . . P71,P135

Tsoller D ................... P82

Turcotte K . . . . . . . . . . . . . P144,P145

Turner $\mathrm{L} \ldots \ldots \ldots \ldots \ldots \ldots \ldots$. P84

\section{$\mathrm{U}$}

Uddin Z ................. $16 \mathrm{H}$

Uleryk E ................... P128

Uman LS ................ P18

\section{$\mathrm{V}$}

Valeri BO ................. P146,P147

Vallabh P .................. 8B 
Van Eerd D ................. P56

Van Lieshout RJ. . . . . . . . . . . . 16I,P92

Velly A ..................... P114

Venable C .................... P36

Vernon $\mathrm{H} \ldots \ldots \ldots \ldots \ldots \ldots \ldots . . \ldots \ldots$

Verrier M .................. P118

Vervoort T .................. P148

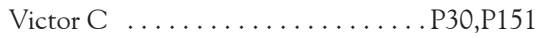

Villeneuve E ................... P103

Vinall J ..................... P149

Vinette J ................. P142

\section{W}

Walco $\mathrm{G}$ P139

Walco GA $12 \mathrm{C}$
Walker LS . P136

Walker S 9B,P10

Walsh Z . P54

Walter SD . $\mathrm{P} 28$

Ware MA $\ldots \ldots \ldots \ldots \ldots \ldots$ 7,P114,P150

Ware $\mathrm{M} \ldots \ldots \ldots \ldots \ldots 7 \mathrm{~A}, 16 \mathrm{G}, \mathrm{P} 88, \mathrm{P} 93$

Warr D $\mathrm{P} 58, \mathrm{P} 153$

Wasylenko E ................ 26B

Watt-Watson J . . . . . . . . . . P151

Waxman J . . . . . . . . . . . . . . 161,P92

Weinberg EL ................. P114

Weisman SJ . . . . . . . . . . . . . . P81

Welkom JS ................. P132

Wideman TH............ 5,5A,20A

Williamson OD ............... 13,P114
Wilson AC. .................... P65

Wilson KG.................. P96

Wright D .................. P66

$\mathrm{Y}$

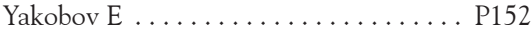

Yamada J .................. P30,P126

Young A $\ldots \ldots \ldots \ldots \ldots \ldots \ldots . . \ldots 153$

Z

Zahran A .................. P26

Zee R .................... P45

Zimmermann C .............. P58,P153

Zoheiry N ................ P4,P154

Zwicker JG................... P149 


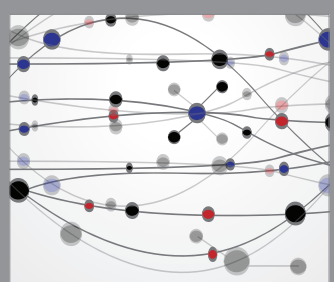

The Scientific World Journal
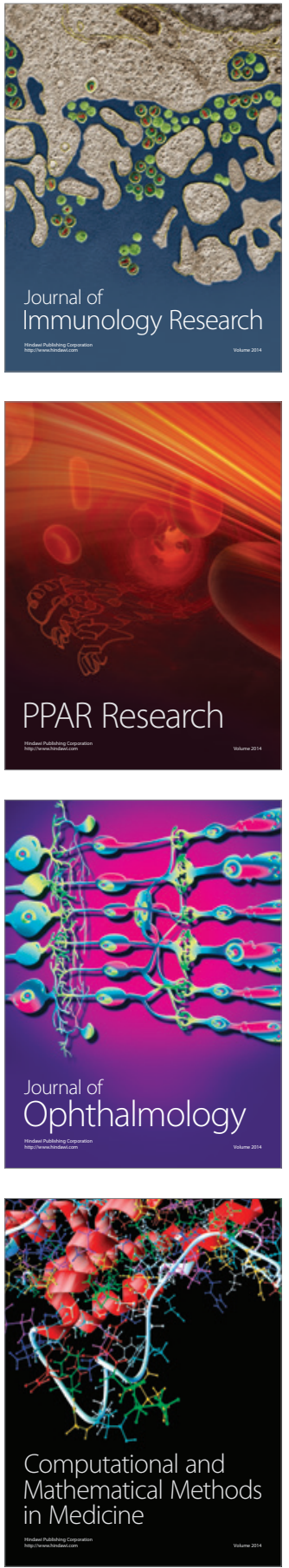

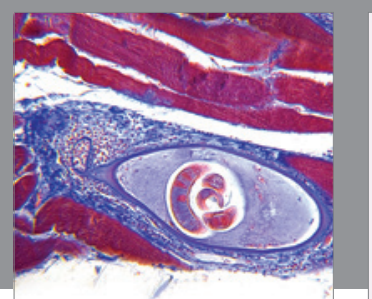

Gastroenterology Research and Practice

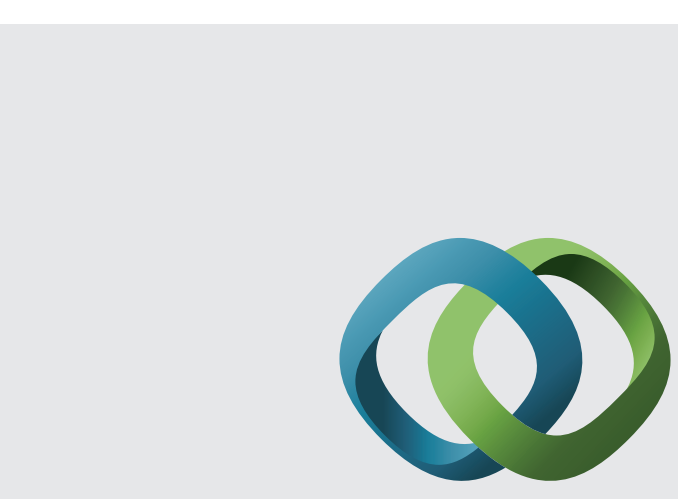

\section{Hindawi}

Submit your manuscripts at

http://www.hindawi.com
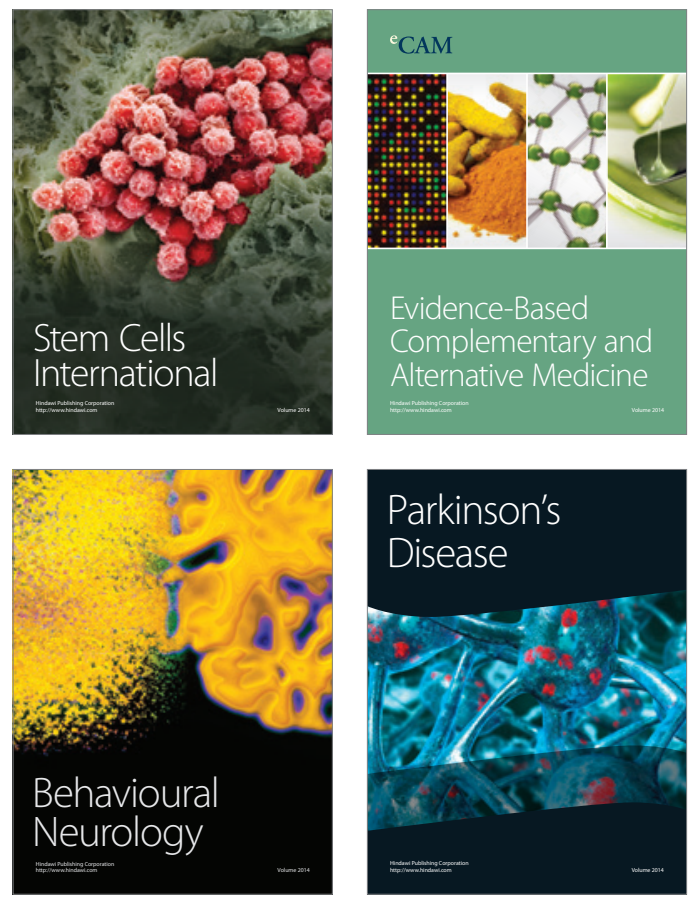
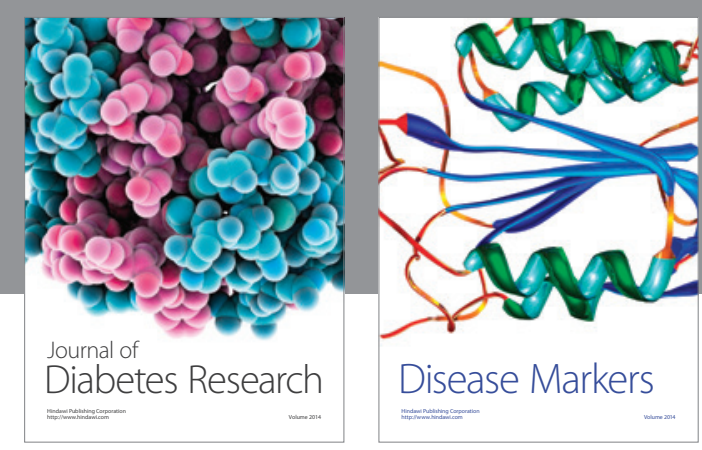

Disease Markers
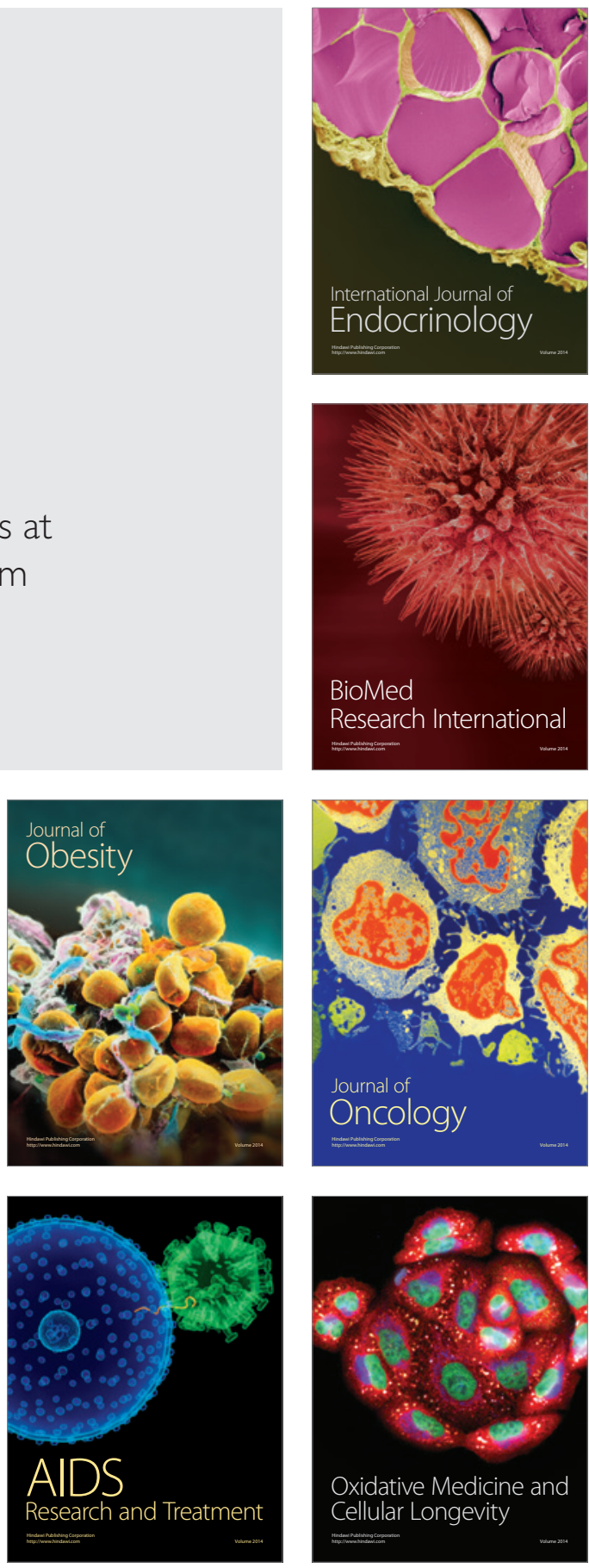